This document is downloaded from DR-NTU (https://dr.ntu.edu.sg) Nanyang Technological University, Singapore.

\title{
Engineering G-quadruplex DNA : a combined computational and experimental study
}

Lech, Christopher Jacques

2013

http://hdl.handle.net/10356/55380

https://doi.org/10.32657/10356/55380 

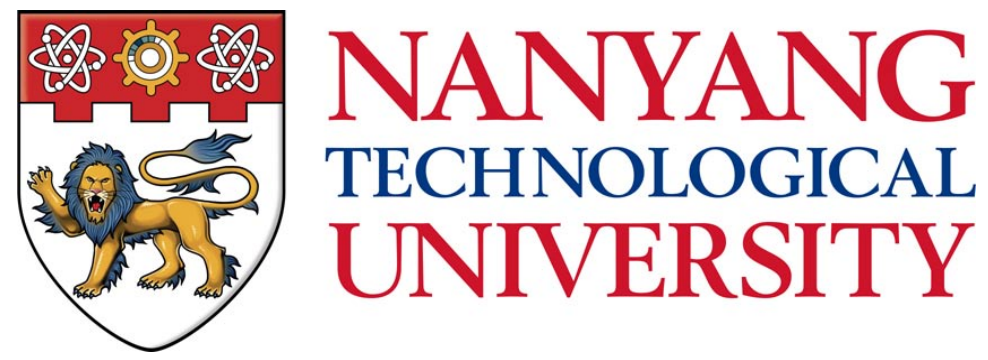

\section{ENGINEERING G-QUADRUPLEX DNA: A COMBINED COMPUTATIONAL AND EXPERIMENTAL STUDY}

by

CHRISTOPHER JACQUES LECH

SCHOOL OF PHYSICAL AND MATHEMATICAL SCIENCES 

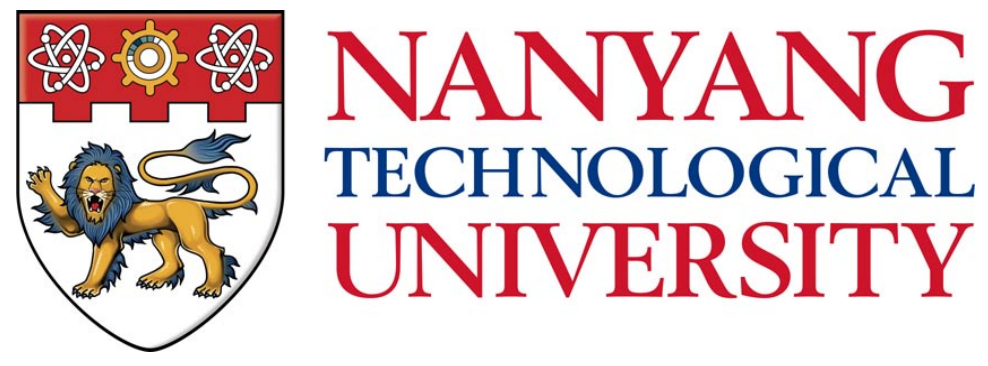

\section{ENGINEERING G-QUADRUPLEX DNA: A COMBINED COMPUTATIONAL AND EXPERIMENTAL STUDY}

by

CHRISTOPHER JACQUES LECH

SCHOOL OF PHYSICAL AND MATHEMATICAL SCIENCES 2013

A thesis submitted to the Nanyang Technological University in partial fulfillment of the requirement for the degree of Doctor of Philosophy 


\section{Acknowledgments}

First and foremost, I would like to thank Anh Tuân Phan for all of his guidance over the course of my studies. Through his patience and understanding, he has been an educator in many respects. I am grateful to have had the opportunity to be his student.

I also would like to thank all current and previous lab members and collaborators. Science is most enjoyable when enjoyed with others. The collaborative problem solving I have had the pleasure to undertake during my studies has been the highlight of my research experiences. A particular thanks to Anh Tuan Phan, Brahim Heddi, Michael Adrian, Thuan Nguyen, and Alexander Voityuk for the stimulating scientific discussions. I also thank my colleagues who have helped to train and teach me over the years, particularly Brahim Heddi and Juliet Tan. I am also incredibly thankful to the undergraduate students I have had the pleasure of mentoring and working with over my studies, particularly Zhe Li who I have worked with for many years.

I give a special thanks to all of my family and friends. Without them, nothing is possible.

Lastly, I would like to acknowledge the Singapore International Graduate Award (SINGA) for supporting my graduate studies. 


\section{Contents}

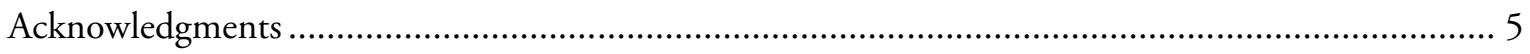

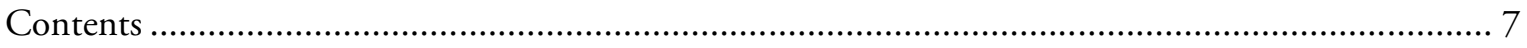

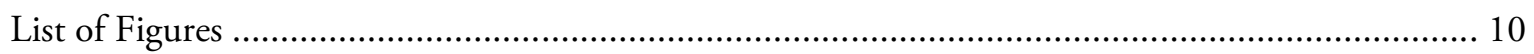

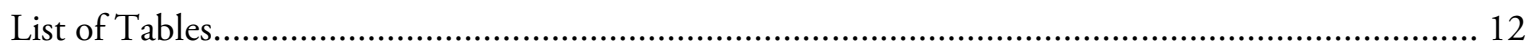

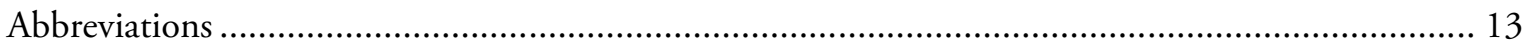

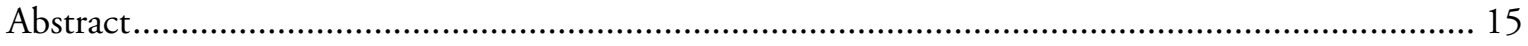

Chapter 1: Introduction to G-quadruplex Nucleic Acids ............................................................. 18

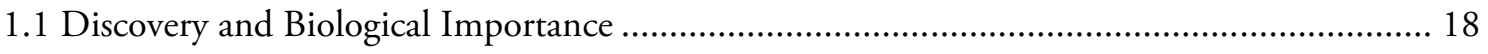

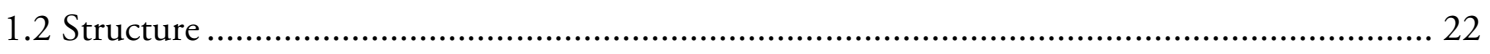

1.2.1 Guanine-tetrads and the G-quadruplex core …............................................................ 22

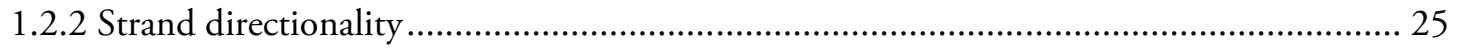

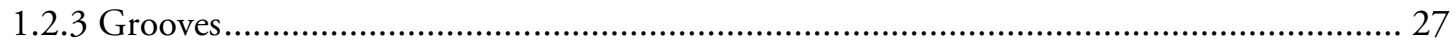

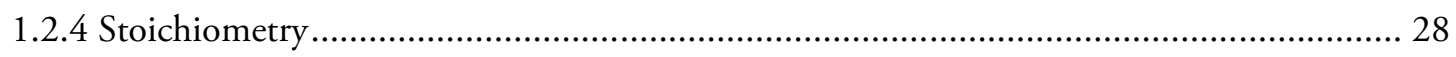

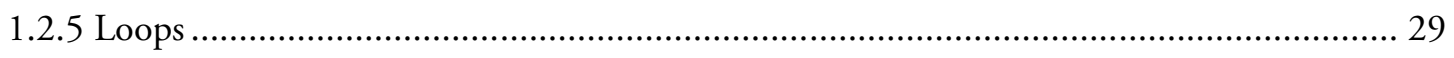

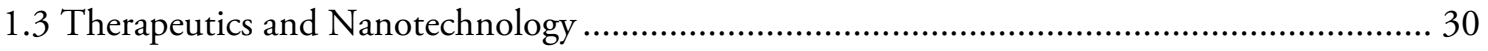

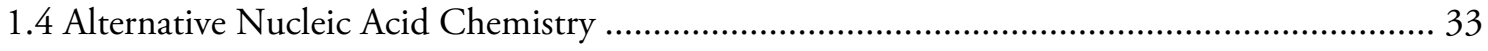

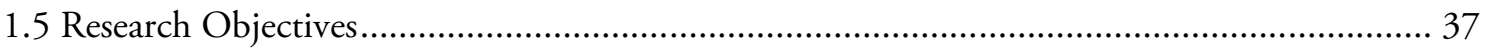

Chapter 2: Fundamentals of G-quadruplexes: Base Stacking and High-Order Assemblies ............. 39

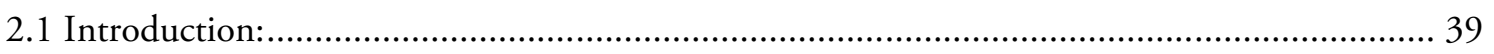

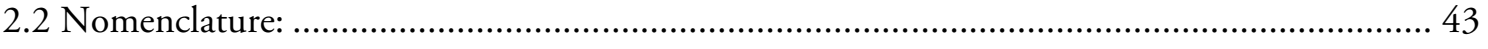

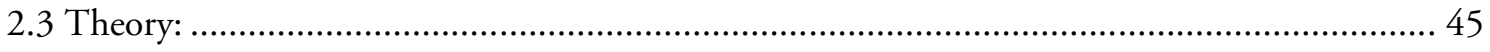

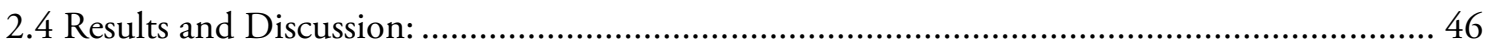

2.4.1: Structural survey and characterization: Guanine stacking within the G-tetrad core ........ 46

2.4.2: Structural survey and characterization: Guanine stacking at the G-tetrad interface of

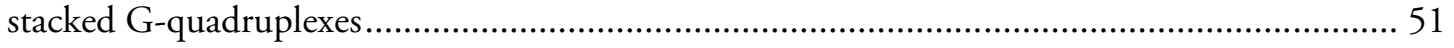

2.4.3: QM computational studies: Energy landscapes of K+ coordinated stacked G-tetrads ..... 53

2.4.4: Molecular Mechanics energy landscapes of stacked G-tetrads ........................................ 59

2.4.5: Investigating 3'-3' and 5'-5' G-quadruplex stacking using Molecular Dynamics ............ 62

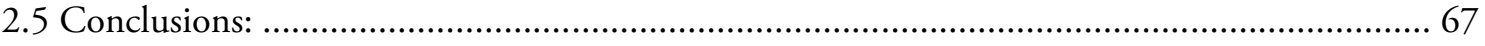

Chapter 3: Fundamentals of G-quadruplexes: Charge Transport ...................................................... 69

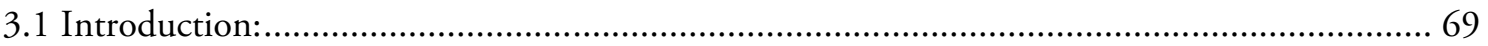




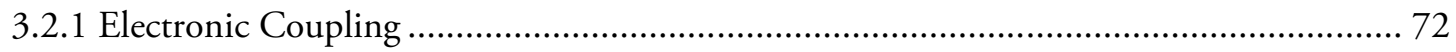

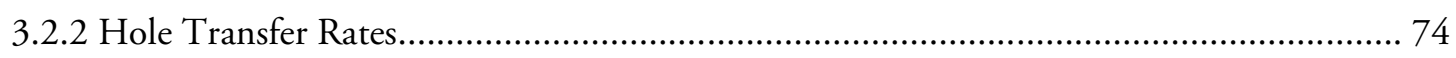

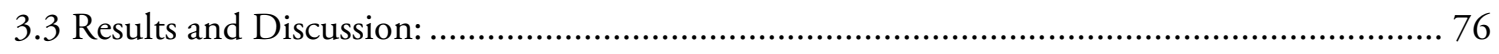

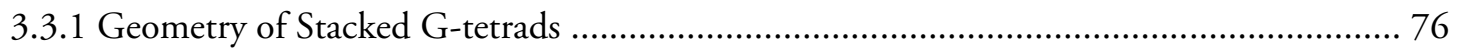

3.3.2 Electronic coupling and hole transfer rates .................................................................. 79

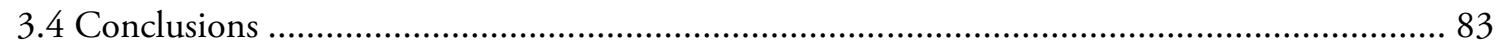

Chapter 4: Engineering the Sugar Backbone: Locked Nucleic Acid ............................................. 84

4.1 Introduction:

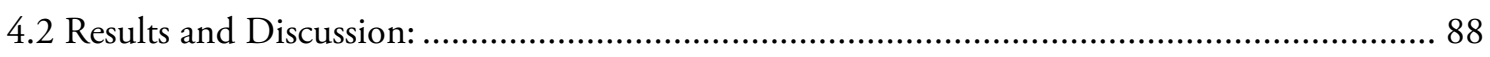

4.2.1: Substitution of ${ }^{\mathrm{LNA}} \mathrm{G}$ into a (4+0) G-quadruplex: LNA modifications are detrimental

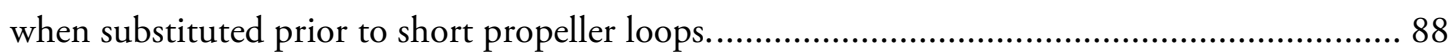

4.2.2 Substitution of ${ }^{\mathrm{LNA}} \mathrm{G}$ into a $(3+1) \mathrm{G}$-quadruplex: LNA modifications generally affect structure in a syn/anti dependent manner............................................................................. 91

4.2.3 LNA modification induces local backbone rearrangement: NMR solution structure of a $(3+1)$ G-quadruplex containing a single ${ }^{\mathrm{LNA}} \mathrm{G}$ modification ................................................ 95

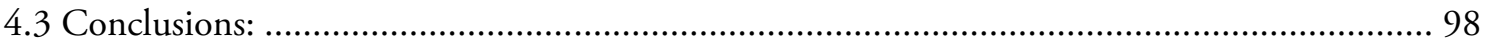

Chapter 5: Engineering the Sugar Backbone: 2'-F-Guanosine and 2'-F-ANA-Guanosine

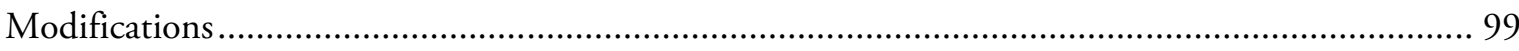

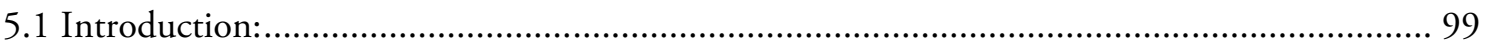

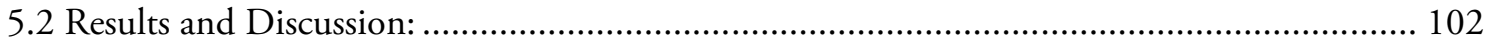

5.2.1: 2'-F-guanosine and 2'-F-ANA-guanosine substitutions destabilize syn positions and are

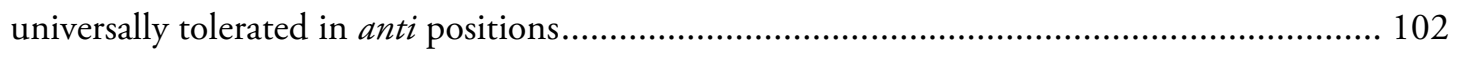

5.2.2: 2'-F-ANA-guanosine substitutions stabilize G-quadruplex DNA while 2'-F-guanosine

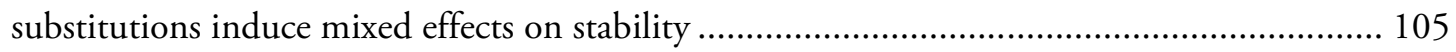

5.2.3: ${ }^{\mathrm{F}} \mathrm{G}$ and ${ }^{\mathrm{FANA}} \mathrm{G}$ are powerful tools for structural manipulation of G-quadruplex ............ 107

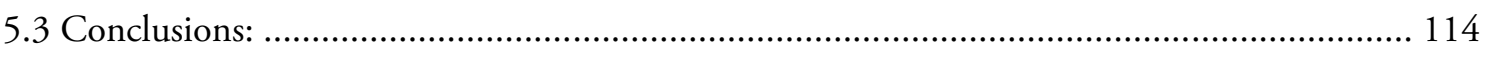

Chapter 6: Engineering the Base: C8-modified Guanines........................................................ 115

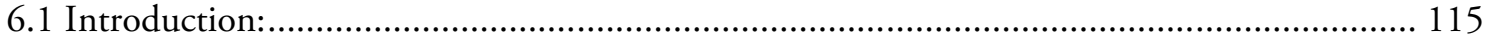

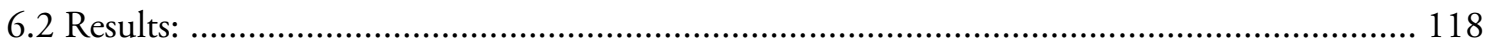

6.2.1: Site-specific substitutions of a guanine with an 8-bromo-guanine, 8-O-methyl-guanine, 8-

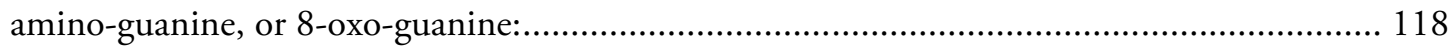

6.2.2: Thermal stability of modified sequences ........................................................................ 120

6.2.3: A single guanine substitution in a G-tetrad results in specific patterns of NMR chemical shift changes: Experimental observations 
6.2.4: A single guanine substitution in a G-tetrad results in specific patterns of NMR chemical shift changes: Computational studies.

6.2.5: Structural effects of C8-modifications on G-tetrad geometry: An ab initio computational study

6.3 Discussion:

6.4 Conclusion:

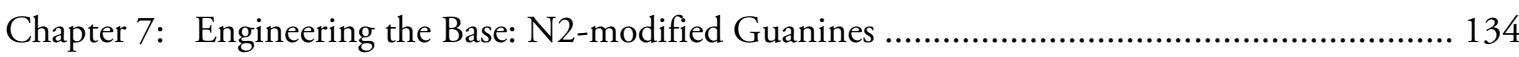

7.1 Introduction:

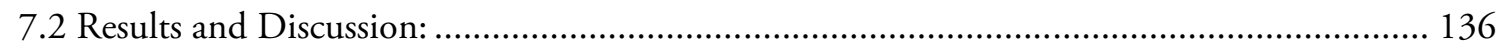

7.2.1: G-quadruplex nanoparticle formation of sequences containing single N2-guanine

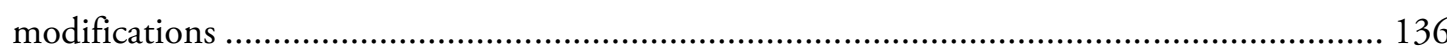

7.2.2: N2-modified G-quadruplexes display enhanced thermal stability ............................... 141

7.2.3: Design of highly stabilized G-quadruplex nanoparticles............................................... 143

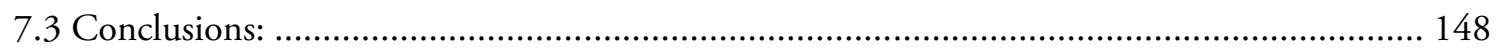

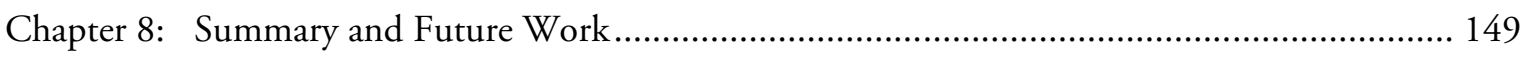

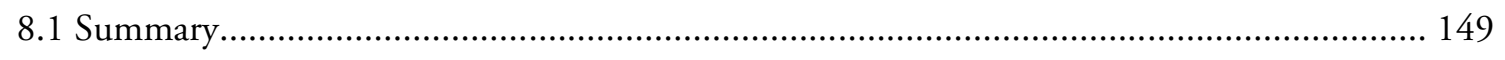

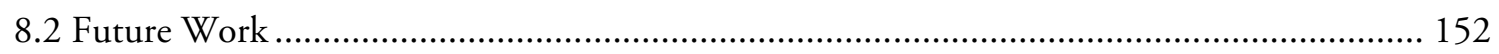

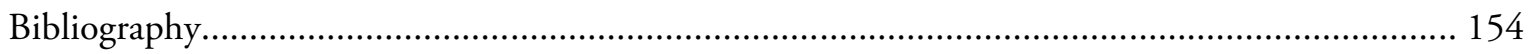

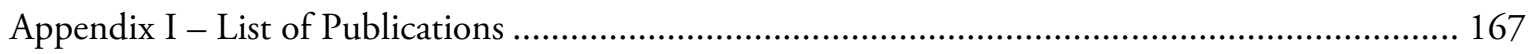

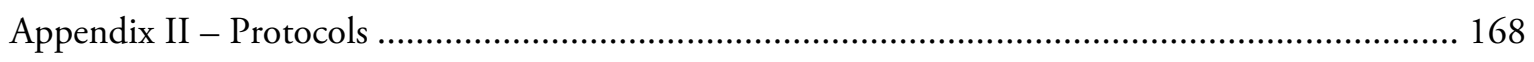

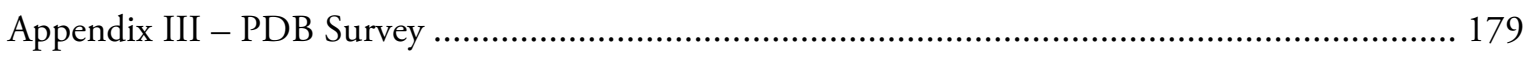




\section{List of Figures}

\section{Chapter 1: Introduction to G-quadruplex Nucleic Acids}

Figure 1-1: Schematic of the Watson-Crick double helix

Figure 1-2: Targeting biological G-quadruplexes as an anti-cancer strategy

Figure 1-3: Schematic of a guanine tetrad

Figure 1-4: Hoogsteen hydrogen bond polarity and G-tetrad stacking orientation

Figure 1-5: G-tetrad stacking modes

Figure 1-6: G-quadruplex stem and glycosidic base conformation

Figure 1-7: Strand directionality

Figure 1-8: Grooves

Figure 1-9: Stoichiometry

Figure 1-10: Commonly occurring loops of G-quadruplexes

Figure 1-11: G-quadruplex structures identified with pharmacological properties

Figure 1-12: Schematic of modified nucleic acid backbone chemistries

\section{Chapter 2: Fundamentals of G-quadruplexes: Base Stacking and High-Order Assemblies}

Figure 2-1: G-tetrad stacking types

Figure 2-2: Illustrative examples of core base stacking modes from the structural survey

Figure 2-3: Histograms of characterization parameters

Figure 2-4: Illustrative examples of G-tetrad base stacking modes at the interface of stacked G-quadruplexes

Figure 2-5: QM energy landscapes

Figure 2-6: Cation influence on QM energy profiles

Figure 2-7: $\mathrm{MM}$ energy landscapes

Figure 2-8: MD investigation of G-quadruplex stacking interfaces

Figure 2-9: Illustrative interface geometry of MD trajectories

\section{Chapter 3: Fundamentals of G-quadruplexes: Charge Transport}

Figure 3-1: Introduction to Stacking polarity and computational models

Figure 3-2: G-tetrad stacking modes of study

Figure 3-3: Evolution of electronic coupling with time and dependence on twist angle

Figure 3-4: Relative electron hole transfer rates

\section{Chapter 4: Engineering the Sugar Backbone: Locked Nucleic Acid}

Figure 4-1: Introduction to LNA and G-quadruplexes studied in this chapter Figure 4-2: Structural characterization of a parallel LNA-modified G-quadruplex Figure 4-3: Thermal stability of a Parallel LNA-modified G-quadruplexes Figure 4-4: Loop expansion experiments

Figure 4-5: Syn/anti dependence of LNA-modification on (3+1) G-quadruplex structure Figure 4-6: Thermal stability of LNA-modified $(3+1)$ G-quadruplexes Figure 4-7: Exceptions to the syn/anti stability dependence of LNA Figure 4-8: NMR solution structure of a LNA-modified $(3+1)$ G-quadruplex Figure 4-9: Dihedral angle analysis of the unmodified and LNA-modified (3+1) Gquadruplex NMR structure 


\section{Chapter 5: Engineering the Sugar Backbone: 2'-F-Guanosine and 2'- F-ANA-Guanosine Modifications}

Figure 5-1: Modified nucleosides and G-quadruplex scaffolds studied in this chapter

Figure 5-2: Effect of glycosidic conformation on ${ }^{\mathrm{F}} \mathrm{G}$ and ${ }^{\mathrm{FANA}} \mathrm{G}$ substitutions into the $(3+1)$

G-quadruplex

Figure 5-3: Incorporation of ${ }^{\mathrm{F}} \mathrm{G}$ and ${ }^{\mathrm{FANA}} \mathrm{G}$ substitutions into the (4+0) G-quadruplex scaffold

Figure 5-4: Change in the melting temperature for single position ${ }^{\mathrm{F}} \mathrm{G}$ and ${ }^{\mathrm{FANA}} \mathrm{G}$ substitutions Figure 5-5: Approach to structural manipulation via ${ }^{\mathrm{F}} \mathrm{G}$ and ${ }^{\mathrm{FANA}} \mathrm{G}$ modifications

Figure 5-6: Decreasing polymorphism using ${ }^{\mathrm{FANA}} \mathrm{G}$ modifications

Figure 5-7: Decreasing polymorphism using ${ }^{\mathrm{F}} \mathrm{G}$ modifications

Figure 5-8: Conformational switching using ${ }^{\text {FANA }} \mathrm{G}$ modifications

Figure 5-9: Conformational switching using ${ }^{\mathrm{F}} \mathrm{G}$ modifications

\section{Chapter 6: Engineering the Base: C8-modified Guanines}

Figure 6-1: Introduction to C8-modified bases

Figure 6-2: CD spectra of C8-modified sequences

Figure 6-3: NMR spectra of C8-modified sequences

Figure 6-4: Effects of C8-guanine substitutions on thermal stability

Figure 6-5: Monitoring NMR chemical shifts of C8-modified tetrads using ${ }^{15} \mathrm{~N}$-labeling experiments

Figure 6-6: Comparison of experimental and computed NMR chemical shift changes

\section{Chapter 7: Engineering the Base: N2-modified Guanines}

Figure 7-1: G-quadruplex scaffolds and N2-modifications explored in this chapter

Figure 7-2: G-quadruplex nanoparticles containing single N2-modifications

Figure 7-3: Select N2-modifications to a parallel G-quadruplex scaffold

Figure 7-4: Design of highly stable G-quadruplex nanoparticles containing multiple N2modifications

Figure 7-5: Design of a "Ball with Hair" G-quadruplex nanoparticle.

Figure 7-6: Model of the "Ball with Hair" G-quadruplex.

\section{Appendix II: Protocols}

Figure A2-1: Characterization Algorithm for Chapter 2 survey of experimental structures 


\section{List of Tables}

Chapter 2: Fundamentals of G-quadruplexes: Base Stacking and High-Order Assemblies

Table 2-1: Statistics of base stacking modes as observed in experimentally determined structures

Table 2-2: Statistical analysis of structural and environmental variables

Table 2-3: Calculated tetrad stacking energies of experimentally observed stacking modes

\section{Chapter 3: Fundamentals of G-quadruplexes: Charge Transport}

Table 3-1: Geometrical characterization of MD G-tetrad stacks

Table 3-2: Electronic coupling between different G-tetrad stacked

Table 3-3: Electron hole rates

Table 3-4: Electronic coupling between guanines within a single G-tetrad

\section{Chapter 4: Engineering the Sugar Backbone: Locked Nucleic Acid}

Table 4-1: LNA-modified sequences studied in this chapter

\section{Chapter 5: Engineering the Sugar Backbone: 2'-F-Guanosine and 2'- F-ANA-Guanosine Modifications}

Table 5-1: DNA Sequences and single-position thermal stability data

Table 5-2: Thermal stability analysis of engineered sequences

\section{Chapter 6: Engineering the Base: C8-modified Guanines}

Table 6-1: List of DNA sequences examined in this work

Table 6-2: Melting temperatures of C8-modified sequences

Table 6-3: Experimentally observed changes in imino proton NMR chemical shifts

Table 6-4: Computed change in imino proton NMR chemical shift

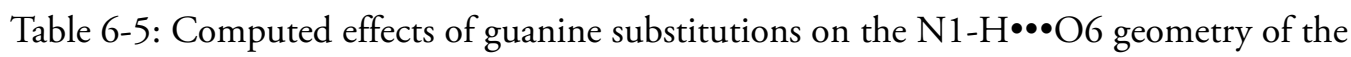
tetrad

Table 6-6: Computed effects of guanine substitutions on the N2-H $\bullet \bullet N 7$ geometry of the tetrad

\section{Chapter 7: Engineering the Base: N2-modified Guanines}

Table 7-1: Sequences containing single-position N2-modifications

Table 7-2: Biophysical characterization of N2-modified sequences

Table 7-3: Change in melting temperature upon single-position N2-modifications

Table 7-4: Thermal stability of sequences containing multiple N2-modifications

\section{Appendix III: PDB Survey}

Table A3-1: Summary and statistics of crystallographic structures cataloged in Chapter 2.

Table A3-2: Summary and statistics of NMR structures cataloged in Chapter 2. 


\section{Abbreviations}

93-del G-quadruplex-forming sequence with anti-HIV activity

A Adenine

AS1411 G-quadruplex-forming sequence with anti-cancer activity

C Cytosine

CD Circular cichroism

CT Charge transfer

$\mathbf{D}_{\mathbf{2}} \mathbf{O} \quad$ Deuterium oxide

DFT Density functional theory

DNA Deoxyribonucleic acid

dsDNA Double-stranded deoxyribonucleic acid

D.S.S. 4,4-dimethyl-4-silapentane-1-sulfonic acid

ET Electron transfer

FCD Fragment charge difference

FRET Förster resonance energy transfer

G Guanine

G-tetrad Guanine tetrad

G-wire Long molecular wire arranged of stacked G-tetrads

${ }^{A m} \mathbf{G}$ 8-amino-guanosine

${ }^{\text {Ben }} \mathbf{G} \quad$ N2-benzyl-guanine

${ }^{\mathrm{Br}} \mathbf{G}$ 8-bromo-guanine

${ }^{\mathbf{F}} \mathbf{G}$ 2'-F-guanosine

${ }^{\text {FANA }} \mathbf{G}$ '-F-ANA-guanosine

${ }^{H e x} \mathbf{G} \quad$ N2-6-amino-hexyl-guanine

${ }^{\text {LNA }}$ Gocked nucleic acid modified guanosine

${ }^{\text {Met }} \mathbf{G} \quad$ N2-methyl-guanosine

${ }^{\text {Omet }} \mathbf{G}$ 8-O-methyl-guanine

${ }^{\text {Oxo }} \mathbf{G} \quad$ 8-oxo-guanine

HIV Human immunodeficiency virus

HMBC Heteronuclear multiple bond correlation

HSQC Heteronuclear single quantum correlation

HT Hole transfer

KPi Potassium phosphate

LNA Locked nucleic acid

MD Molecular dynamics

MM Molecular mechanics

MP2 $\quad 2^{\text {nd }}$ Order Møller-Plesset perturbation theory

NMR Nuclear magnetic resonance

NOE Nuclear overhauser effect

NOESY Nuclear overhauser effect spectroscopy

NT Nucleotide

PDB Protein data bank

PNA Peptide nucleic acid

PPM parts per million

QM Quantum mechanics

RMSD Root-mean-square deviation

RNA Ribonucleic acid

SD Standard deviation 
SELEX Systematic evolution of ligands by exponential enrichment

T Thymine

$\mathbf{T}_{\mathbf{m}} \quad$ Melting temperature

TBA Thrombin-binding aptamer

TERRA Telomeric repeat-containing RNA

TOCSY Total correlation spectroscopy

UNA Unlocked nucleic acid

UTR Untranslated region

UV Ultraviolet

$\boldsymbol{V} \quad$ Electronic coupling 


\section{Abstract}

G-quadruplex nucleic acids are four-stranded structures that arise from guanine-rich sequences. G-quadruplexes have received a great deal of research attention for their biological roles, therapeutic potential, and roles in nanotechnology. The research of this thesis is dedicated to the engineering of G-quadruplex DNA structures. This is done through a mixture of experimental and computational approaches. We examine some of the fundamental properties that govern G-quadruplex structure and their high-order assemblies. We also investigate the application of alternative nucleic acid chemistries within G-quadruplex structures. These include modifications to the base and the sugar moiety of the deoxyribose-phosphate backbone. We demonstrate how these chemical tools may be used to enhance the properties G-quadruplexes and apply these tools in our efforts to engineer G-quadruplex molecules.

The stacking of G-tetrads in the core of G-quadruplexes is a defining feature of these nucleic acid structures. Stacked G-tetrads can adopt a variety of different geometries. We perform a survey and characterization of experimental G-tetrad stacking geometries published in the literature, identifying unique G-tetrad stacking geometries with the core and at the interface of stacked G-quadruplexes. QM computational approaches are used to calculate the G-tetrad energy landscapes, providing an understanding of the energy differences between different G-tetrad stacking orientations. All atom MD simulations of stacked G-quadruplexes provide an understanding for the preferential orientation of stacked G-quadruplexes observed in the literature and highlight important consideration for the engineering of stacked G-quadruplex systems.

Long strands of stacked G-tetrads offer a unique avenue for the generation of conductive 
wires on the nanoscale (G-wires). Currently, there is little understanding of how the orientation of stacked G-tetrads affects the migration of charge through the G-tetrad core. We characterize the ground state electronic properties of different G-tetrad stacking arrangements. Large samples of molecular geometries are extracted from MD simulations. We demonstrate that electron hole delocalization and electron transfer rates vary significantly for different G-tetrad stacking geometries.

Modification of DNA by alternative chemistries can enhance the thermal stability of oligonucleotides as well as their resistance to enzyme degradation. We examine the guanine analog containing a locked nucleic acid (LNA) chemical modification $\left({ }^{\mathrm{LNA}} \mathrm{G}\right)$ in the context of G-quadruplex. The formation of G-quadruplex and the thermal stability of resulting structures are characterized. We question old assumptions of effective ${ }^{\mathrm{LNA}} \mathrm{G}$ substitution and report on novel limitations of ${ }^{\mathrm{LNA}} \mathrm{G}$ incorporations into G-quadruplex DNA. To explore the structural effects of ${ }^{\mathrm{LNA}} \mathrm{G}$ substitutions, we solve the high-resolution NMR-solution structure of a (3+1) G-quadruplex containing a single ${ }^{\text {LNA }} \mathrm{G}$ modification.

In a similar manner, we study the effects of 2'-deoxy-2'-fluoro-riboguanosine and 2'deoxy-2'-fluoro-arabinoguanosine modification into G-quadruplex. Receiving less research attention then LNA, we seek to define the rules which govern effective incorporation of these nucleotides via a systematic study on multiple G-quadruplex platforms. We also explore the ability to modulate G-quadruplex folding topology based on the observed glycosidic preference of these modified nucleotides and their rational substitution into G-quadruplex forming sequences.

Modification of the guanine base at the C8-position has been previously demonstrated to favor a syn-conformation. We examine the site-specific incorporation of 8-bromo-guanine, 
8-O-methyl-guanine, 8-amino-guanine, and 8-oxo-guanine. We demonstrate the sitespecific and chemical-specific nature of the effects these modifications have structure and stability. We report on the novel effects of these modifications on NMR chemical shifts, and relate these observations to changes in G-tetrad hydrogen bond geometry through QM studies.

Modification at the N2-position of the guanine base has received little research attention to date. We show that such modifications generally stabilize the G-quadruplex to a high degree. The projection of N2-modification into the groove represents a novel way to functionalize the G-quadruplex in a spatially-specific manner. We design a novel and highly stable "Ball-with-hair" G-quadruplex-based nanoparticle for general use as functionalizable and thermally stable nanoparticle scaffold.

The work presented here will be valuable to the molecular design of novel G-quadruplex structures. It will also enable the engineering and optimization of existing G-quadruplexes scaffolds. 


\section{Chapter 1:}

\section{Introduction to G-quadruplex Nucleic Acids}

\subsection{Discovery and Biological Importance}

In 1953, the interpretation of X-ray crystallographic data by Watson and Crick revealed the first correct model of "double helix" DNA (1-3). However, in 1910, long before the discovery of the double helix, it was observed that guanine mono-phosphate was capable of forming gels (4). This curious observation was later explained in 1962 by Gellert and colleagues when crystallographic structures of these aggregates demonstrated the formation guanine-tetrads (5), later realized to be the building block of the G-quadruplex structures. Since the first crystallographic and NMR solution structures of G-quadruplex DNA in the early 1990's (6-8), roughly 150 atomic-resolution structures have collectively demonstrated the diversity of this unique class of nucleic acids. The interest in Gquadruplex structures has been largely fueled by their various suggested roles in biological processes $(9,10)$. The identification of proteins that recognize and unwind Gquadruplex structures along with the recent visualization of G-quadruplexes within human cells highlights the growing consensus on the biological importance of these nucleic acid structures (11-13). 


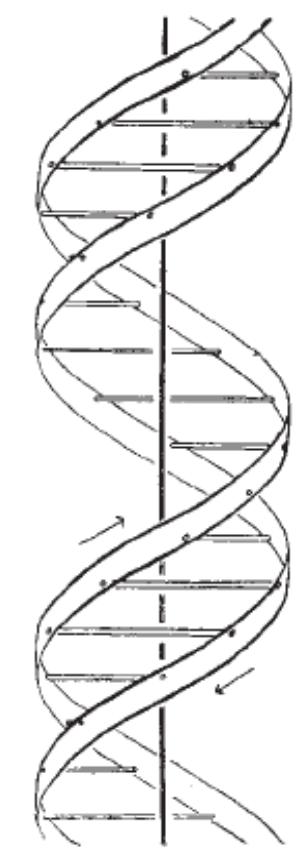

Figure 1-1: Schematic of the Watson-crick double helix proposed in 1953. Sourced from (1)

The human telomere is a region of the genome which acts as a protective cap at the end of chromosomes. It is characterized by tandem repeats of the sequence (TTAGGG) $)_{n}$ and contains a single stranded 3'-end overhang $(10,14)$. Due to an inability of DNA polymerase to completely replicate the telomere, telomeric DNA shortens after each cell division $(15,16)$. The enzyme telomerase counter-acts this shortening by extending the telomere (17-19), but it is not normally activated in somatic cells (20). It was hypothesized that the formation of G-quadruplex in this G-rich region may play a role in telomere stability and maintaining genome integrity. Human telomeric sequences have been shown to form a variety of G-quadruplexes in crystal and solution environments $(6,21-33)$.

Gene promoter regions of the human genome also contain G-rich sequences capable of forming G-quadruplexes. In fact, bioinformatics surveys of the human genome have shown that over $40 \%$ of gene promoter regions are thought to contain G-quadruplex 
forming sequences (34). This number is significantly higher for the promoter regions of oncogenes (35), which are genes whose mutation or over expression are associated with cancer. Alternatively, this number is much lower for tumor suppressor genes (36). Gquadruplex structures formed by numerous oncogenic sequences in vitro have been solved to date (37).

G-quadruplex-forming sequences are also prevalent in the 5'-untranslated regions (UTRs) of messenger RNAs $(10,38)$. These non-coding regions play important regulatory roles in translation. Bioinformatics studies of the human genome have shown these regions to contain a high number of G-quadruplex forming motifs compared to other transcribed areas of genome (39), including numerous proto-oncogenes. The formation of Gquadruplex in 5'-UTRs can inhibit $(40,41)$ and in some cases enhance (42) translation.

The realization that G-quadruplex formation occurs in genomic regions important to cancer proliferation has sparked interest in targeting these structures as an anti-cancer strategy. The enzyme telomerase responsible for telomere elongation is over expressed in a majority of cancers (43). As G-quadruplexes have been shown to inhibit this extension (44), the induction of telomeric G-quadruplex formation by small molecules is one such anti-cancer strategy (45) (Fig 1-2A). Another feature of many cancers is the overexpression of oncogenes. The induced formation of G-quadruplex in oncogenic promoter regions, to inhibit oncogene expression, represents another anti-cancer strategy involving the drug-targeting of biologically important G-quadruplexes (37) (Fig 1-2B). Considering these strategies, many small molecules have been identified to target G-quadruplexes (46). While many of these compounds demonstrate high binding affinity to G-quadruplex, only some demonstrate selectivity over duplex DNA and no compounds have been identified that are highly selective among different G-quadruplexes forming sequences. 
(A)

$$
\text { Targeting Telomeres }
$$
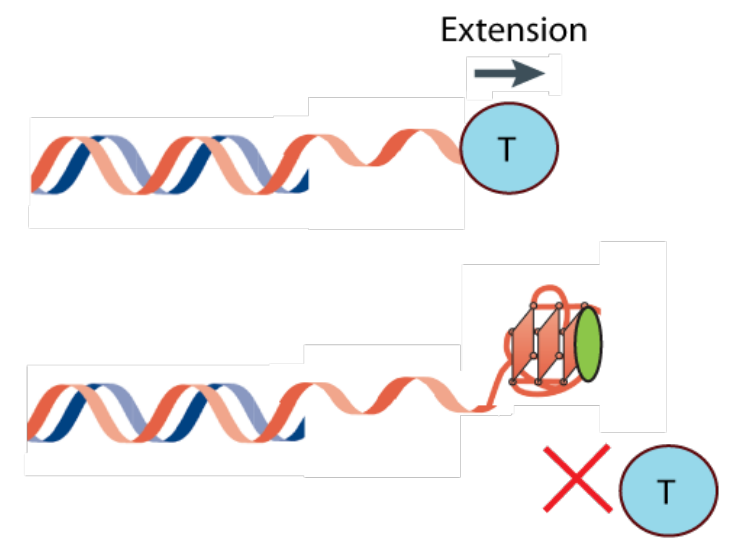

(B)

\section{Targeting Oncogenes}
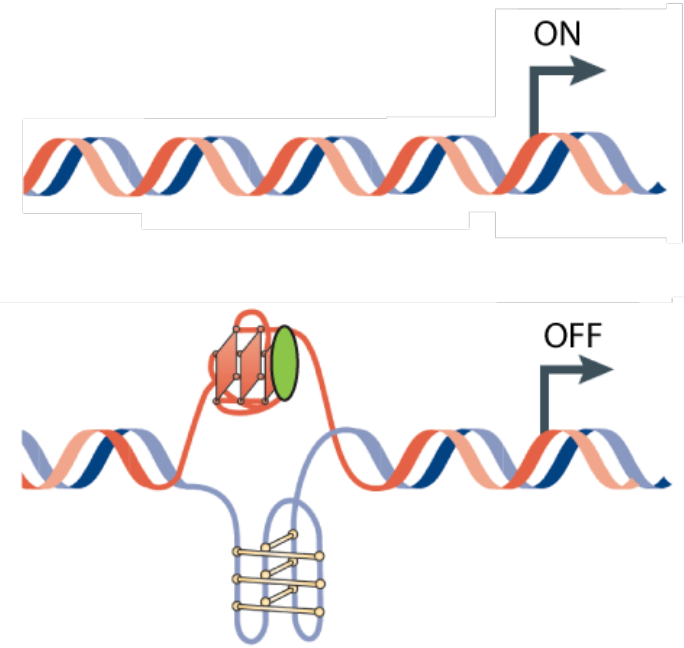

Figure 1-2: Anti-cancer strategies targeting G-quadruplex DNA with small molecules: (A) Telomerase (light blue), over expressed in the majority of cancers, elongates the telomere creating cell immortality. The induction of G-quadruplex formation in the single stranded telomeric overhang via small molecules (green) blocks telomere extension by telomerase and is a potential anti-cancer strategy. Figure adapted from (37). (B) The formation of G-quadruplex and i-motif (C-quadruplex) within the promoter regions is suspected to occur under super-coiled DNA during the transcription process. The stabilization of G-quadruplex structures with small molecules (green) blocks transcription of over expressed oncogenes as a potential anticancer strategy. Figure adapted from (37).

In addition to their roles in telomere maintenance and gene regulation as discussed above, G-quadruplexes are suspected of playing other important biological roles (47). G-rich RNA which are transcriptional products of telomeric and sub-telomeric DNA are capable of forming G-quadruplex structures $(48,49)$ and are thought to play protective and regulatory roles in the telomere (50). Additionally, the formation of DNA G-quadruplexes in non-coding regions of the genome has been shown to affect replication and recombination (51,52), inducing genomic instability and gross chromosomal rearrangement in yeast $(52,53)$. 


\subsection{Structure}

\subsubsection{Guanine-tetrads and the G-quadruplex core}

The guanine-tetrad is a fundamental building block of the G-quadruplex architecture. Also termed G-tetrads, these roughly planar assemblies are found in the interior of Gquadruplex structures. G-tetrads arise when four guanine bases form Hoogsteen hydrogen bonds in a cyclical pattern (Fig 1-3). During G-tetrad formation, the N2-H (amino proton) and N1-H (imino proton) of one guanine base bonds with the N7 and O6 position, respectively, of its neighbor. This association usually occurs in the presence of cations that stabilize G-tetrad formation through coordination with the O6-position of the guanine bases (54). The formation of G-quadruplex is sensitive to ion concentration and chemistry (55-57). Different ions types stabilize G-quadruplex formation to different extents. For example, guanine-rich sequences that readily assemble in $\mathrm{K}^{+}$or $\mathrm{Na}^{+}$solution are often observed to not form G-quadruplexes in the presence of $\mathrm{Li}^{+}$(58). The size difference of various ions can also influence the mobility and coordination sites within the G-tetrad core.

Within the core of a G-quadruplex structure, multiple G-tetrads stack upon one another, with the guanine base from one G-tetrad stacked with a guanine from a neighboring tetrad. In most cases, guanines that are stacking partners are also consecutive bases within the G-quadruplex forming sequence. For example the first and second guanine of the sequence (GGGTTA) ${ }_{4}$ will most likely stack upon one another in neighboring G-tetrads. However, in some more complex structures this is not the case.

The geometry of stacked G-tetrads can vary significantly, affecting both the stability of 


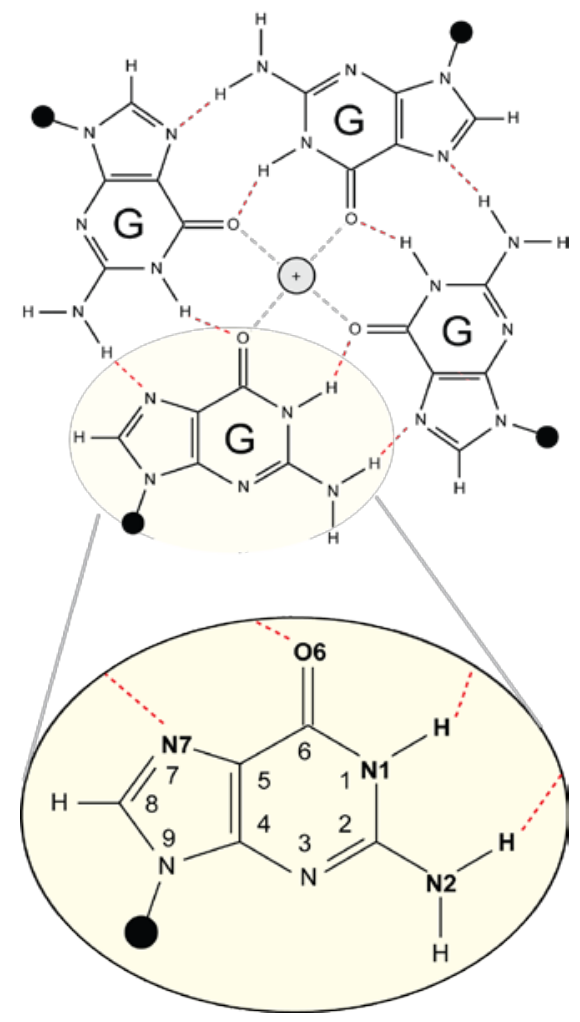

Figure 1-3: Schematic of a guanine tetrad: Hoogsteen hydrogen bond (red) and ion coordination (grey) patterns are shown. Magnified view of guanine highlights common atomic naming convention.

the G-tetrad stack and the geometry at the exterior of the molecules that interacts with solvent (59). The cyclic nature of the hydrogen bond pattern in a G-tetrad gives rise to a specific donor-to-accepter directionality and can serve as a convenient way for defining the geometry of stacked G-tetrads (Fig 1-4A). Based on this definition of polarity, two neighboring G-tetrads can stack with their polarities in the same direction, 'same-polarity stacking', or opposite direction, 'opposite-polarity stacking'. In an alternative nomenclature, the donor-to-accepter directionality defines a G-tetrad face, with positive out-of-plane projection being the "head" and negative being the "tail” according to righthanded vector notation (60). G-tetrads may then be stacked in a "Head-to-Head”, "Tailto-Tail” or “Head-to-Tail” manner.

While, in principle, stacked G-tetrads may adopt many different geometries when rotated 
(A)

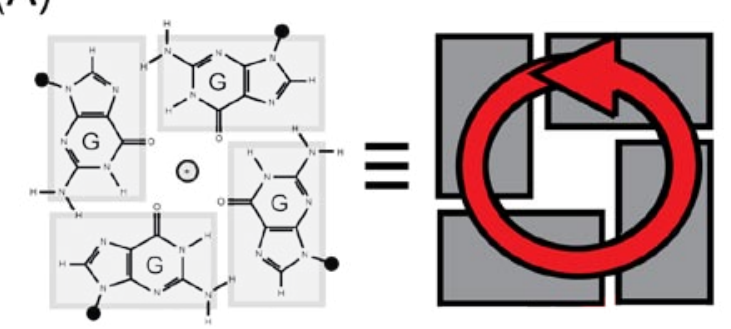

(B)
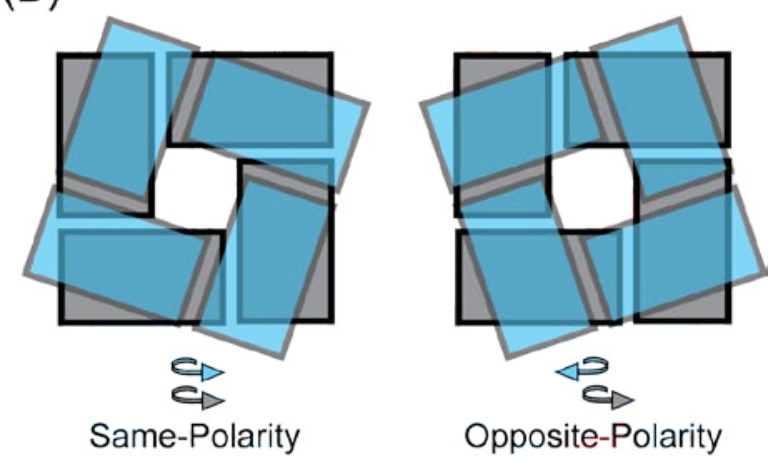

Figure 1-4: (A) Hydrogen-bond polarity of the guanine-tetrad as defined by the $\mathrm{N} 1-\mathrm{H} \rightarrow \mathrm{O} 6$ or $\mathrm{N} 2-\mathrm{H} \rightarrow \mathrm{N} 7$ direction. (B) Schematic examples of the 'same-polarity' and 'opposite-polarity' orientation of two stacked guanine-tetrads

with respect to each other, only specific stacking conformations are observed experimentally. This is due to covalent linkage between the guanine base and the G-tetrad backbone. Three separate G-tetrad stacking geometries are observed within the G-tetrad core of G-quadruplexes (61). The Partial 5/6-ring geometry is adopted when G-tetrads stack in a same-polarity manner, with guanine bases exhibiting a partial overlap of a 5member ring with a 6-member ring (Fig 1-5A). The 5-ring and the Partial 6-ring stacking geometries can arise when G-tetrads stack in an opposite polarity manner, with stacked guanines displaying overlap of their 5-member rings and a partial overlap of their 6member rings respectively (Fig 1-5B-C). The geometries adopted within the G-tetrad core of the G-quadruplex are intrinsically linked to the glycosidic conformation of the base (discussed below). 
(A)

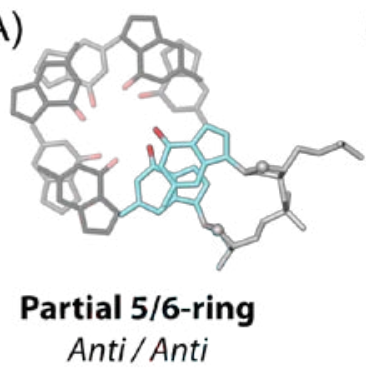

(B)

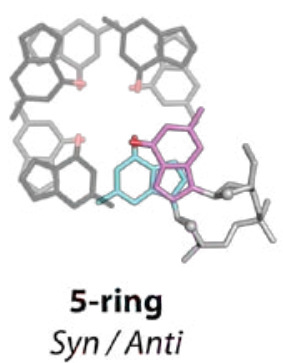

(C)

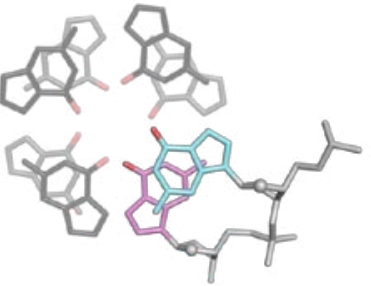

Partial 6-ring Anti/Syn
(D)

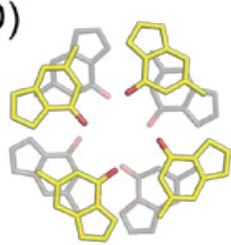

Partial 6-Ring
(E)

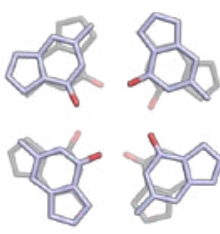

6-Ring
(F)

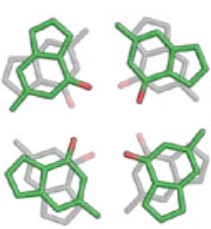

5/6-Ring
(G)

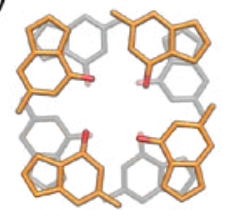

5-Ring

Figure 1-5: G-tetrad stacking modes: (A-C) G-tetrad stacking geometries found within the core of Gquadruplexes. Guanines bases of different polarities are colored syn and magenta in relation to their glycosidic conformation. (See "1.2.2 Strand Directionality" section). (D-G) G-tetrad stacking geometries observed at the interface of higher-order G-quadruplex assemblies

The stacking of G-tetrads can also occur in higher order aggregates of G-quadruplex. Two G-quadruplex monomer units are sometimes observed to stack upon one another at their G-tetrad interface. This dimerization can result in unique G-tetrad stacking geometries at the stacking interface, some of which are not found within the core of covalently constrained G-tetrads (Fig 1-5D-G) (21,62-64). These geometries are also named after the relative overlap of their aromatic rings. Some of these geometries are also observed in unique conformations of dimeric G-quadruplexes (65).

\subsubsection{Strand directionality}

In a strand of DNA, guanine bases are covalently attached at the N9-position of the base to the ribose sugar of the phosphate-sugar backbone. In G-quadruplexes, guanine bases that participate in G-tetrad formation usually adopt two different glycosidic conformations with respect to sugar as defined by the dihedral angle O4'-C1'-N9-C4 (Fig 1-6A). The conformational ranges adopted by the base are a defining characteristic 
(A)

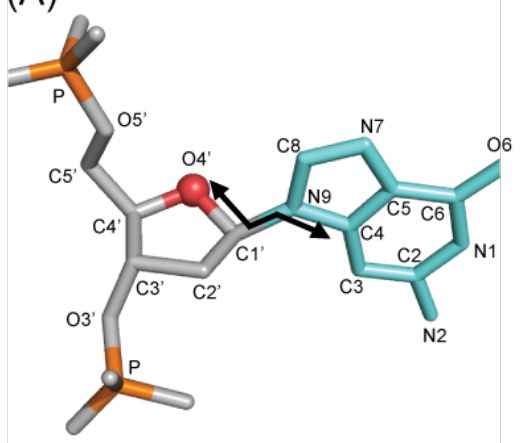

(B)

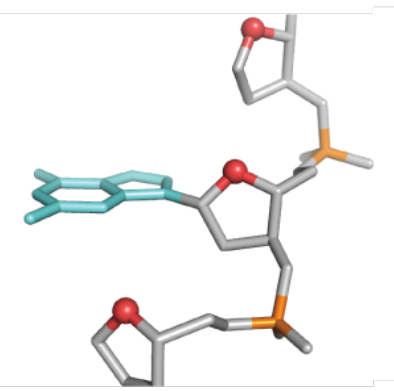

(C)

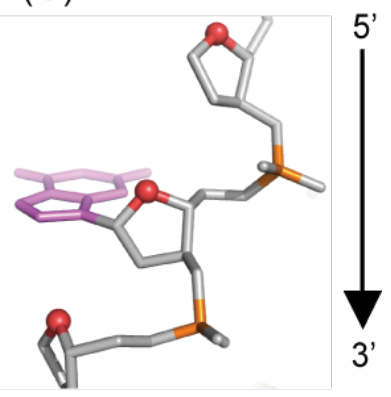

Figure 1-6: The G-quadruplex strand and guanine base glycosidic conformation: (A) Atomic naming convention for guanosine nucleotides. The glycosidic conformation is described by the O4'-C1'-N9-C4 dihedral angle as indicated. Examples of an (B) anti and (C) syn glycosidic base conformation are presented. The strand directionality of the G-quadruplex stem is defined in the $5^{\prime} \rightarrow 3^{\prime}$ direction. Backbone and sugar atoms are generally represented as grey with the exceptions of phosphorous (orange) and the O4' of the sugar (red). Guanine base colors correspond to an anti (cyan) or syn (magenta) conformation.

of the overall G-quadruplex structure, and are usually found within an "anti” $-120^{\circ} \rightarrow 180^{\circ}$ or "syn" $0^{\circ} \rightarrow 90^{\circ}$ conformational range (Fig 1-6B-C) (66). The asymmetry of the sugarphosphate backbone can be used to define a strand directionality. The directionality from C5' to the C3' atom of the backbone is a commonly regarded reference for positive strand directionality, often simply described as " 5 ' $\rightarrow 3$ '” (Fig 1-6).

The directionality of the strands within a G-quadruplex can vary in orientation (Fig 1-7). When all strands point in the same direction, the G-quadruplex folding topology is referred to as being in a "parallel" or a " $(4+0)$ ” orientation. The “ $(3+1)$ ” or "hybrid" orientation is formed when one strand is oriented in the opposite-direction of the other
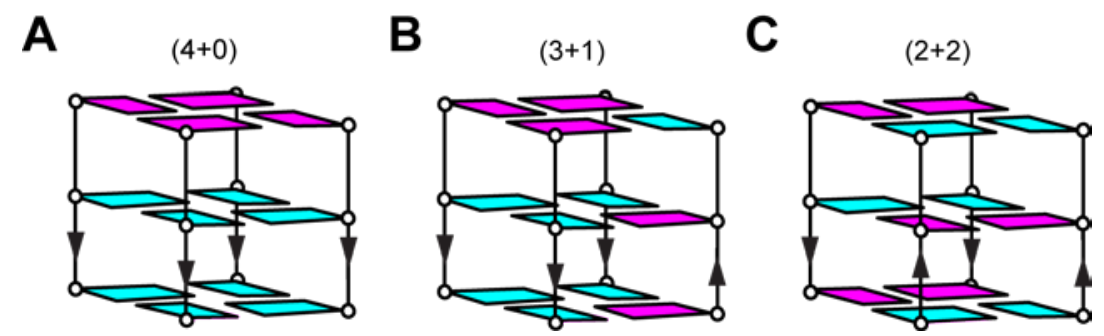

Figure 1-7: Strand directionality within the G-quadruplex core: (A) Parallel “(4+0)” strand orientation with all strands point in the same direction. (B) Hybrid " $(3+1)$ " folding with three strands in one direction and one in the opposite. (C) Anti-parallel " (2+2) orientation, with 2 strands pointing in each direction. Guanines are colored based on their anti (cyan) or syn (magenta) glycosidic conformation. The relative glycosidic conformation of guanines within a G-tetrad are related to the strand directionality. 
three strands. In a similar manner, an "anti-parallel” or “(2+2)” orientation defines a Gquadruplex formed by two strands pointing in one direction, and two in the other. In most G-quadruplex structures, the glycosidic conformations of guanines within a single Gtetrad are directly related to the relative strand-orientations of the G-tetrad core. When all strands point in the same direction, all guanines within a G-tetrad adopt the same glycosidic conformation. The re-orientation of one or two strand leads to a differing glycosidic conformations on these strands (Fig 1-7).

\subsubsection{Grooves}

Similar to double helix DNA, the sugar-phosphate backbone strands that connect guanines of the G-tetrads give rise to grooves. These grooves, or concave regions between

(A)

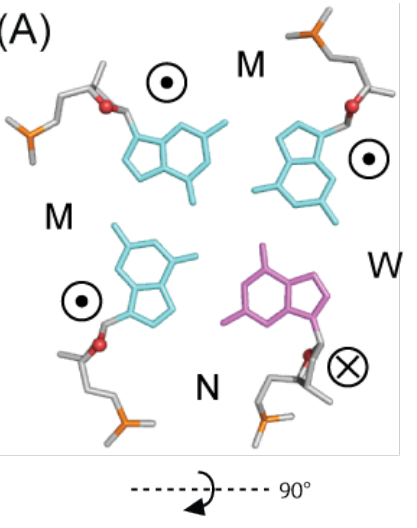

Narrow

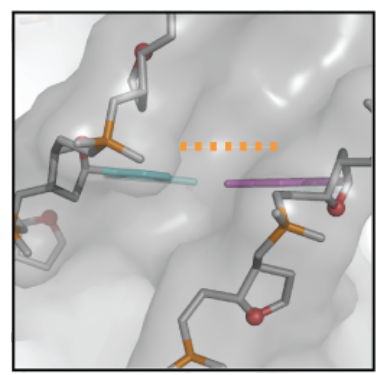

(B)

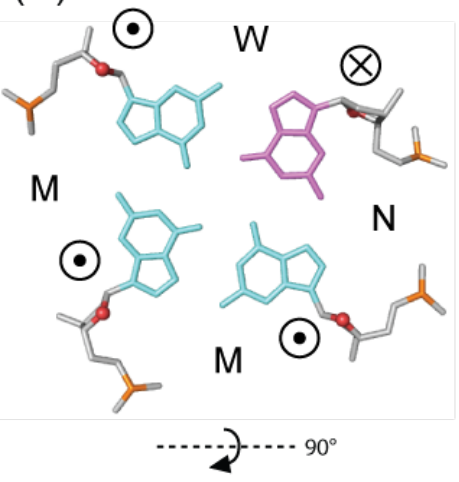

Medium

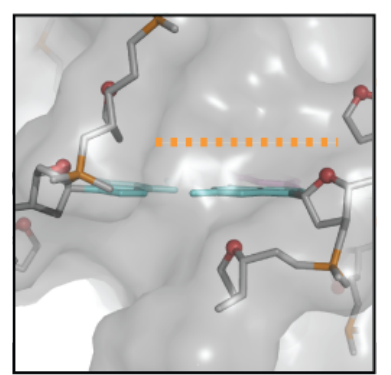

(C)

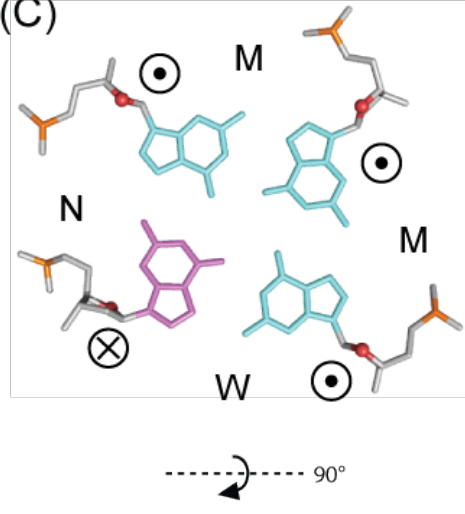

Wide

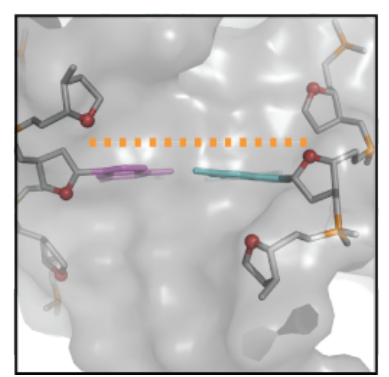

Figure 1-8: Groove widths of G-quadruplex: Groove widths are commonly referred to as (A) Narrow, (B) Medium or (C) Wide. Grooves of different widths arise from various glycosidic conformations of bases within the G-tetrad. (Top) guanine bases are colored based on their anti (cyan) or syn (magenta) glycosidic conformation. Phosphorous (Orange) and O4' atoms (red) are colored for clarity. Strand directionality is referenced as into $(\mathrm{X})$ or out of $(\bullet)$ the page. Groove widths for the presented strand orientation are abbreviated as Narrow (N), Medium (M), and Wide (W). (Bottom) Illustrative examples of grooves widths: Surface representations are shown with a qualitative measure of groove width indicated (orange dotted line). 
the sugar-phosphate backbones, can vary in dimensions depending on the nucleic acid conformation. In G-quadruplex DNA, The four strands that make up the stem of the Gquadruplex give rise to four grooves, the dimensions of which depend on the directionality of the strands (Fig 1-8) $(67,68)$. If all of the strands in the stem are oriented in the same direction as in a (4+0) parallel structure (Fig 1-7A) then four grooves of "Medium” width will be formed. The orientation of one strand in an opposite direction such as in the $(3+1)$ hybrid (Fig 1-7B) will generate a narrow and a medium groove (Fig 1-8). G-quadruplex grooves are an important feature of the G-quadruplex structure as small-molecules have been identified which interact with G-quadruplex grooves $(46,69,70)$.

\subsubsection{Stoichiometry}

The four strands of a G-quadruplex may assemble from different parts of a single guanine-rich G-quadruplex forming sequence, or from the conjunction of multiple sequences. The stoichiometry of a G-quadruplex, or the number of sequences from which it is made, may be unimolecular, bimolecular, or tetrameric (Fig 1-9).

(A)

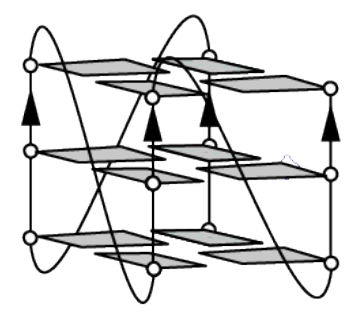

Unimolecular
(B)

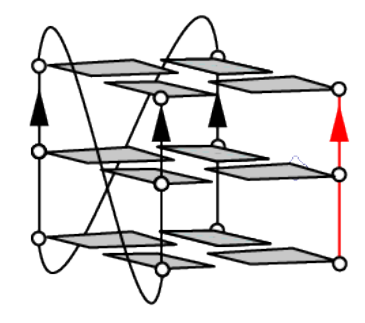

Bimolecular

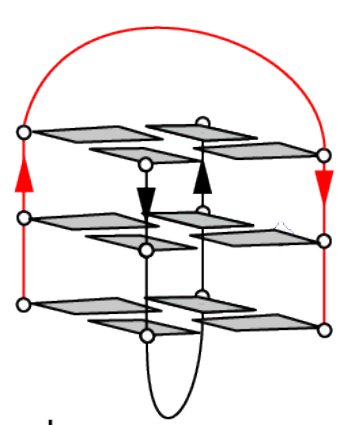

(C)

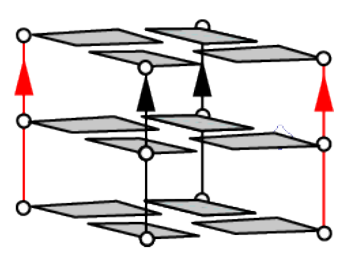

Tetramolecular

Figure 1-9: Stoichiometry of G-quadruplex DNA: G-quadruplexes can form from (A) a single strand of DNA, (B) two strands or (C) four strands. 


\subsubsection{Loops}

The assembly of guanine rich regions from less than four strands leads to segments nucleic acid that connect strands but do not participate in G-tetrad formation. These segments, called loops, can vary in their length, base chemistry and geometry. There are three commonly occurring loop types, which are classified by the directionality and orientation of the strands they connect (Fig 1-10) A “diagonal” loop connects two strands oriented in opposite directions and located diagonally across from one another in the Gquadruplex. An “edge-wise” loop connects two neighboring strands oriented in opposite directions, while a "propeller” or “double-chain-reversal” loop connects two neighboring strands oriented in the same direction.

(A)

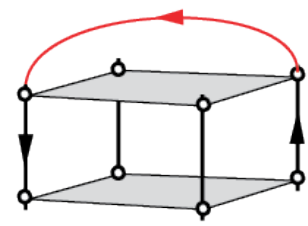

Diagonal
(B)

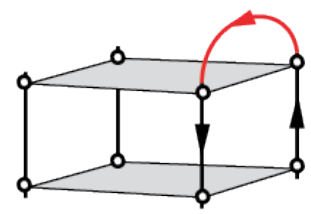

Edge-wise
(C)

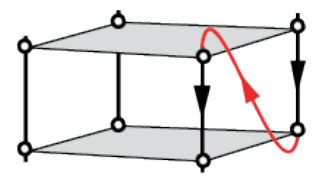

Propeller

Figure 1-10: Commonly occurring loops of G-quadruplex DNA: (A) Diagonal loop (B) Edge-wise loop (C) Propeller loop. Figure adapted from (67).

Loops are a dynamic structural feature of G-quadruplexes $(71,72)$. The geometries adopted by loops vary depending on loop type, length and loop sequence. There are many favorable interactions that can arise from interactions within the loops or between the loops and neighboring structural elements. For example, bases within loops have been observed to stack upon another $(49,73)$ and to hydrogen bond to the edge of a G-tetrad leading to more elaborate planer assemblies such as pentads $(74,75)$. 


\subsection{Therapeutics and Nanotechnology}

The unique geometry and chemical nature of the G-quadruplex scaffold has led to the identification of numerous engineered G-quadruplexes that demonstrate potential use as therapeutics. The discovery of biologically active G-quadruplexes often starts by screening a large library of nucleic acid sequences against a target protein via a SELEX type procedure (76-78), Sequences identified to bind to the protein target with high affinity, termed aptamers, are often further refined by rational engineering of structure.

One of the earliest and most well-studied biologically active G-quadruplexes is the Thrombin-binding aptamer (TBA). The TBA, formed by the sequence $d\left[G_{2} T_{2} G_{2} T G T G_{2} T_{2} G_{2}\right]$, was first identified to be a single stranded binder of the thrombin protease in nanomolar affinity, inhibiting blood coagulation (79). This sequence was later shown to adopt a G-quadruplex structure in X-ray crystallographic and NMR solution studies. (Fig 1-11A) $(80,81)$ Numerous studies involving the optimization and chemical modification of the TBA have demonstrated its potential as a sensing agent (82).

(A)

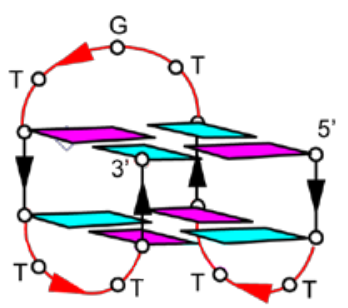

Thrombin Binding Aptamer

Anti-coagulant
(B)

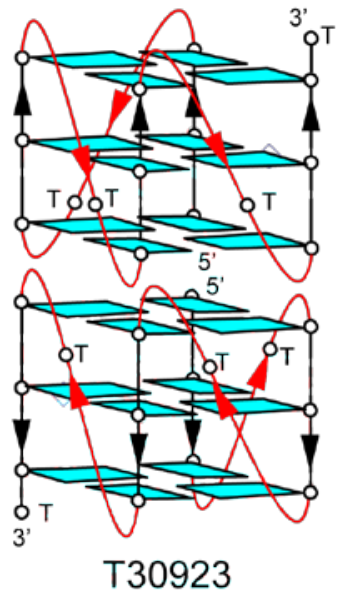

Anti-cancer
(C)

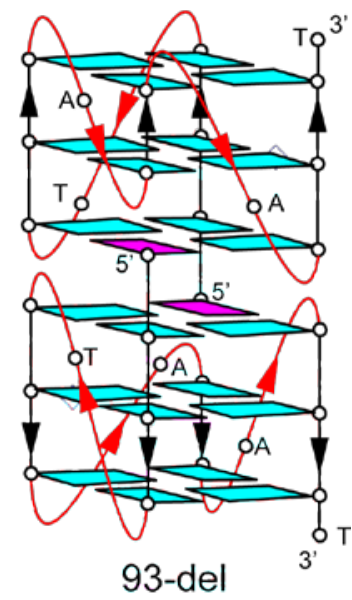

Anti-HIV

Figure 1-11: Scaffolds identified with pharmacological properties: Structural schematics of the (A) Thrombin binding aptamer displaying anti-coagulant activity (B) T30923 displaying anti-cancer activity and (C) 93-del demonstrating anti-HIV1 Integrase activity $(74,80,83)$. 
A series of engineered G-quadruplex structures have been identified that with anti-cancer properties (84,85). The oligonucleotide T30923 and its sequence variants display inhibition of the Stat3 protein, involved in oncogenic signaling in many cancer types, at 10-50 $\mu \mathrm{M}$ affinity. The T30923 sequence has been shown to adopt a dimeric Gquadruplex molecule in solution (83) (Fig 1-11B). Another promising anti-cancer oligonucleotide is the AS1411 sequence which demonstrated anti-cancer effects in numerous cell lines (85). The inhibitory properties of AS1411 as dependent upon Gquadruplex formation (86-88), and it is believed that AS1411 binds to the membrane protein nucleolin (85). However, the exact mechanism of action is not yet clear. The AS1411 sequence is one of the most pharmacologically successful G-quadruplex drugs, currently in Phase II clinical trials.

Numerous G-quadruplex forming sequences have also been identified which demonstrate anti-HIV activity (78). The tetrameric G-quadruplex formed by $d\left(\mathrm{~T}_{2} \mathrm{G}_{4} \mathrm{~T}_{2}\right)$ was one of the first G-quadruplex forming sequences identified with anti-HIV properties (89), binding the HIV envelope protein gp120, a protein crucial to viron-cell membrane fusion, at sub$\mu \mathrm{M}$ concentrations. Another G-quadruplex which displays anti-HIV activity is the 93-del molecule formed from the sequence $d\left(G_{4} T_{3} A_{2} A_{2} G_{3} T\right)$ (90). The 93-del oligonucleotide has been shown in inhibit HIV-I Integrase, which is a protein crucial to incorporation of the viral genome into host cells. This oligonucleotide adopts an interlocked-dimeric Gquadruplex structure (Fig 1-11C) (74). Other G-quadruplex forming sequences of note have been identified to bind to biological targets, but demonstrate less selectivity and pharmaceutical potential then those discussed above (78).

In addition to their role as therapeutic agents, G-quadruplex structures have demonstrated their usefulness in the field of nanotechnology. The self-assembling of guanine- 
derivatives can be used to generate nanopores and grow liquid crystals (54). The Gquadruplex-architecture has also been used to create long wires (G-wires) (91,92). Gwires offer a potential approach to the generation of conductive nanoscale circuitry.

The G-quadruplex architecture is also at the heart of many sensing applications, including sensing of proteins, small molecules, ions, and oligonucleotides $(93,94)$. Some of these sensors are based on G-quadruplex DNA that possesses catalytic activity. These Gquadruplex based deoxyribozymes (DNAzymes) can bind to a target molecule which modulates their catalytic behavior (95-98). One class of structures that has received particular research interest is G-quadruplex based DNAzymes that bind hemin and exhibit peroxidase-like activity (99-102). 


\subsection{Alternative Nucleic Acid Chemistry}

While many G-quadruplex forming molecules have been identified that demonstrate therapeutic potential, native nucleic acid chemistry has limitations. Primarily, native DNA is readily broken down by nuclease within the human body. This is true for G-quadruplex forming DNA as well. The incorporation of alternative nucleic acid chemistries into Gquadruplexes can reduce the effects of enzyme degradation and prolong the lifetime of pharmacologically active G-quadruplex molecules (103-107). Alternative nucleic acid chemistries have many other advantages as well. Incorporation of modified-nucleotide chemistries can stabilize G-quadruplex structure (107). Increasing the stability of pharmacologically active G-quadruplex molecules can lead to increased activity at body temperature simply by increasing the amount of DNA that is forming the desired structure. Incorporation of alternative nucleic acid chemistry can also lead to improved cellular uptake (104).

One approach to modification of nucleic acid is functionalization of the base. In fact, nucleic acid bases often naturally encounter lesions within the cell due to oxidative damage and natural biological processes (108-110). Within the context of G-quadruplexes, modification can be made to bases within the loops or to guanine bases within the Gtetrad core (107). The altering of guanine base chemistry within the G-tetrad core can have profoundly negative effects on G-quadruplex formation if such functionalization disrupts G-tetrad hydrogen bonding $(111,112)$.

There are many locations on the guanine base that may be functionalized or chemically altered (Fig 1-6A). The C8-position is one of the most studied types of guanine base modifications in the context of G-quadruplexes (25,111,113-117). Normally containing a 
hydrogen atom, the C8 position protrudes outward from the core of the G-quadruplex into the groove. While most C8-modifications can be made without hindering G-tetrad formation, some lesions such as 8-oxo-guanine disrupt hydrogen bonding of the G-tetrad via protonation at the N7 position. The proximity of the C8-position to the deoxyribosephosphate backbone makes modification at this position sensitive to the glycosidic conformation of the guanine base (Fig 1-6). Substitutions that are bulkier in nature tend to prefer a syn conformation over an anti orientation of the base $(25,113,118)$. As the folding topology of G-quadruplex is intrinsically related to glycosidic conformation, C8modifications can be used to drive structural changes in G-quadruplex DNA. However, due to this sensitivity, careful use of C8-modifications is required if one desires to maintain to the same global structure following C8-guanine substitution.

Modification at the $\mathrm{O} 6$ and $\mathrm{N} 1$ positions are very limited as these positions are crucial to Hoogsteen bonding and G-tetrad formation. While simple adducts will generally destabilize G-quadruplex formation $(111,112)$, bases that contain the electronegative O6 and N1 substituted with other atoms suitable for Hydrogen-bond formation can be used (111). Careful substitution at positions that hydrogen bond can even allow for the alteration of hydrogen bonding network within the G-tetrad (119).

The N2 position holds an amino group also important for the hydrogen bonding of the Gtetrad. Complete substitution of this group with other chemistries is generally deleterious (111). The replacement of the amino group with a simple hydrogen atom, as in inosine, still allows for hydrogen bonding, but usually at a cost of thermal stability $(111,120,121)$. However, replacement of a single hydrogen atom in the amino group with alkyl and alkyllinked functional groups does not necessarily disrupt G-tetrad formation $(122,123)$. Similar to C8, the N2 position projects out into the grooves of the G-quadruplex and is 
located at a further distance from the deoxyribose-phosphate backbone.

Another approach to modification of G-quadruplex DNA is alteration of the sugar moiety of the deoxyribose-phosphate backbone. This can be particularly helpful to prevent against degradation of the nucleic acid by enzymes (103-106,124). Within the context of G-quadruplex, such modifications can affect the puckering of the sugar moiety, which can affect the preferred glycosidic conformation of the base $(106,124,125)$. Thus, sugarmodified chemistries can also be used to modulate the adopted conformations of Gquadruplex forming sequences. Similar to the incorporation of base-modifications, it is important to consider the structural environments when substituting G-tetrad forming guanines with sugar-modified nucleotides.

One nucleotide analog that has received particular attention is Locked nucleic Acid (LNA). While DNA usually adopts a 2'-endo sugar conformation, LNA contains an O$\mathrm{CH}_{2}$ bridge, linking the $\mathrm{C} 2$ ' and $\mathrm{C} 4$ ' atoms, which forces the sugar moiety into a C3'-endo orientation (Fig 1-12B). LNA-modified guanines generally stabilize structure when substituted into an anti conformation $(65,126,127)$. Different locked sugar chemistries have also been synthesized, but have received less research attention in the context of Gquadruplex (124). Conversely to LNA, unlocked nucleic acid (UNA) contains a broken sugar ring with detachment between the C2' and C3' atoms (Fig 1-12B). This chemistry has mixed effectiveness when substituted into the loops and is very destabilizing when substituted into a G-tetrad (128).

Other modified chemistries that have received slightly less attention than LNA are 2'deoxy-2'-fluoro-riboguanosine (2'-F) and 2'-deoxy-2'-fluoro-arabinoguanosine (2'-FANA) modifications. The substitution of a fluorine atom with one of the two C2' hydrogen atoms grants nuclease resistance to 2'-F- and 2'-F-ANA-containing 

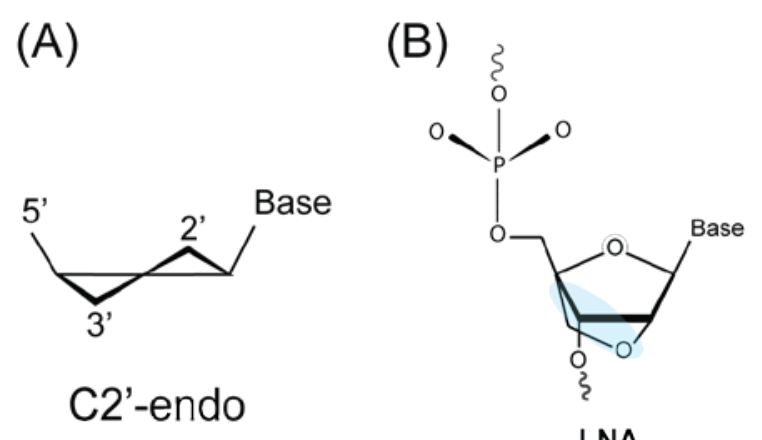

LNA

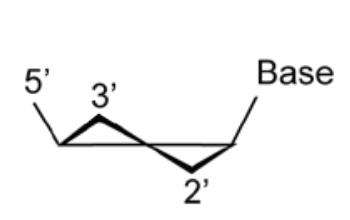

C3'-endo

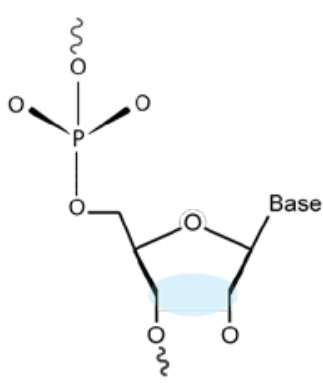

UNA

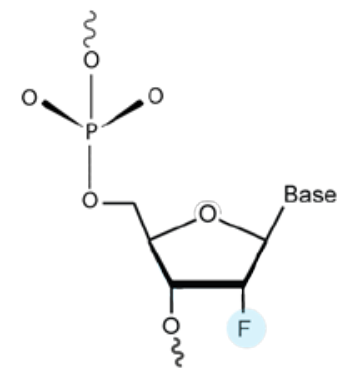

2'-F

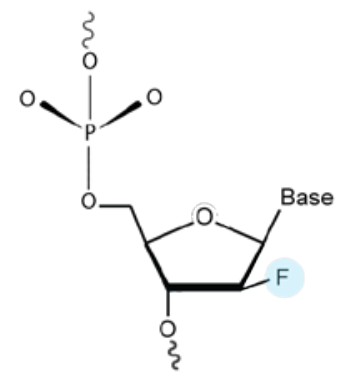

2'-F-ANA
(C)

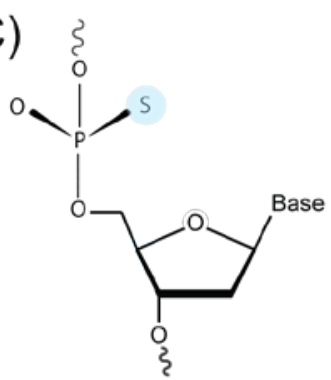

Phosporothiolate

(D)

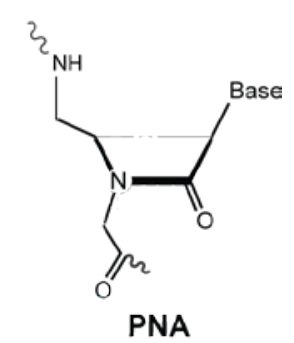

Figure 1-12: (A) Schematic of sugar pucker nomenclature. (B-D) Chemical schematics of nucleotide analogs containing (B) modified sugar, (C) modified phosphate group, (D) peptidic backbone. Areas of deviation from natural DNA are highlighted in light blue.

oligonucleotides $(103,105,106)$. In some respects, 2'-F modified nucleotides mimic RNA nucleotides, which adopt a different preferential sugar pucker (129).

Modification to the phosphate group has also been examined in the context of Gquadruplex DNA. The substitution of one of the phosphate oxygen atoms with a sulfur atom (Fig 1-12C) has a mix of results on the formation and thermal stability of modified G-quadruplexes, with most favorable substitutions being observed within the loops $(130,131)$. In some cases, drastically different analogs have been investigated in Gquadruplex DNA which lack a ribose sugar or phosphate group. Peptide nucleic acid (PNA) is one such analog, which links bases via a peptidic backbone (Fig 1-12D) (132134). A ‘click chemistry approach' has also been previously used to build G-quadruplex structures, replacing the phosphate group with a triazole linker (135). 


\subsection{Research Objectives}

The over-arching goal of the research presented in this Ph.D. thesis is to investigate Gquadruplex structural features and alternative nucleic acid chemistries that enable the design of engineered G-quadruplex structures. These goals can be broken into two main categories.

1. Investigation of the physical interactions in the G-tetrad core of G-quadruplexes

Chapter 2: We investigate how G-tetrad stacking geometry influences structure. As a main building block of G-quadruplex structures, we investigate the roles of G-tetrad stacking in the context of monomeric G-quadruplexes with special interest paid to the roles of stacking at the interface of high-order Gquadruplex assemblies.

Chapter 3: The role of G-tetrad stacking geometry on the electronic properties of the G-tetrad core is considered. Results from this study highlight new structural considerations for the engineering of conductive G-quadruplexbased nanowires.

2. Investigation of alternative nucleic acid chemistries for the design of Gquadruplexes

Chapter 4: Conformationally-restricted locked nucleic acid (LNA) is a useful nucleotide analog that has been previously shown to demonstrate beneficial properties when incorporated into G-quadruplex. We investigate the effects of LNA-modified guanosine on G-quadruplex structure and stability, exploring a variety of structural environments.

Chapter 5: In a similar manner to LNA, we investigate 2'-F-guanosine and 2'-FANA-guanosine. We seek to broaden the understanding of the appropriate 
structural environments for incorporation of these nucleotides into Gquadruplexes.

Chapter 6: Base modification at the C8-position has been previously observed to favor a syn conformation. We investigate 8-bromoguanine, 8-aminoguanine, 8-oxoguanine, and previously unexplored 8-O-methylguanine on Gquadruplex structure and stability as well as their effect on G-tetrad geometry and NMR chemical shifts.

Chapter 7: Modification at the N2-position of the guanine base is investigated for N2-methyl-guanine, N2-6-amino-hexyl-guanine, and N2-benzyl-guanine nucleotide analogs. We also undertake the design of a highly-stable Gquadruplex nanoparticle using a novel approach to G-quadruplex functionalization through N2-modifications. 


\section{Chapter 2:}

\section{Fundamentals of G-quadruplexes: Base Stacking and High-Order Assemblies}

This chapter was largely adapted from the authors work: Lech, C.J., Heddi, B., Phan, A.T. (2013) Guanine base stacking in G-quadruplex Nucleic Acids. Nucleic Acids Research, 41 (3), 2034-2046.

Author Contributions: Survey of the Protein Databank and characterization of experimental structures was carried out by C.J.L.; QM calculations of single and stacked tetrads were undertaken by C.J.L.; MD simulations of stacked G-quadruplexes were performed by C.J.L. and B.H.; The MM parameterization of guanines bases and MM landscape computations were performed by B.H. and C.J.L.; Data analysis was done by C.J.L. and B.H., Experimental and computational approaches were designed by all authors; All authors contributed to the writing of above the manuscript from which this chapter is based.

\subsection{Introduction:}

Study of the physical interactions that govern G-quadruplex formation and their highordered assemblies is important to understanding the structure and function of biologically relevant G-quadruplexes and facilitates the engineering of aptamer molecules. G-quadruplexes are stabilized by a number of physical interactions. Like more canonical nucleic acid structures, the $\pi-\pi$ stacking of aromatic bases is central to the formation of Gquadruplexes. Consequently, a great deal of research has gone into understanding the relative energies of stacked nucleic acid bases in varying geometries (136-141), including G-tetrad geometries found in G-quadruplexes (142-144).

Besides playing a fundamental role in G-quadruplex formation, guanine base stacking plays an important role in higher-ordered G-quadruplex assemblies. Individual G- 
quadruplex units have the capacity to stack upon one another at their G-tetrad interfaces as seen in both X-ray crystallographic $(7,21,62-64,145-150)$ and NMR solution structures $(74,75,83,151-154)$. Unlike in the G-quadruplex core, base stacking geometries at free stacking interfaces are not constrained by covalent linkage through the backbone. Understanding the roles of base stacking at the interface of stacked G-quadruplexes is particularly important considering that high-order assemblies of telomeric G-quadruplex nucleic acid have been observed to form in vitro from naturally occurring telomeric DNA (21) and telomeric repeat-containing RNA (TERRA) $(155,156)$ and that a number of Gquadruplex-based aptamers are formed from stacked G-quadruplexes $(74,83,157)$. With this in mind, we set out to quantitatively characterize the base stacking geometries of Gquadruplexes. We report on the results of an extensive structural survey of G-quadruplex structures published in the Protein Data bank (PDB).

We also investigate base stacking on a fundamental level, examining how G-tetrad stacking energy differs with varying geometry. Previous quantum mechanical (QM) computational studies have looked at the relative stacking energies between some optimized geometries of stacked G-tetrads coordinated with central cations (142-144). The present study expands on previous works by generating comprehensive QM energy landscapes with a large basis set at the MP2 level of theory for both same-polarity and opposite-polarity stacked tetrads by systematically varying the separation and relative rotation of stacked $\mathrm{K}^{+}$coordinated tetrads (Fig 2-1 and Appendix II - Protocols - Chapter 2). Comparison of our experimental survey results and QM energy calculations allows us to understand the relative energies of stacked G-tetrad geometries, both within a single Gquadruplex and at the interface of stacked G-quadruplexes.

We also set out to investigate the role of base stacking in high-order assemblies of G-qua- 
A
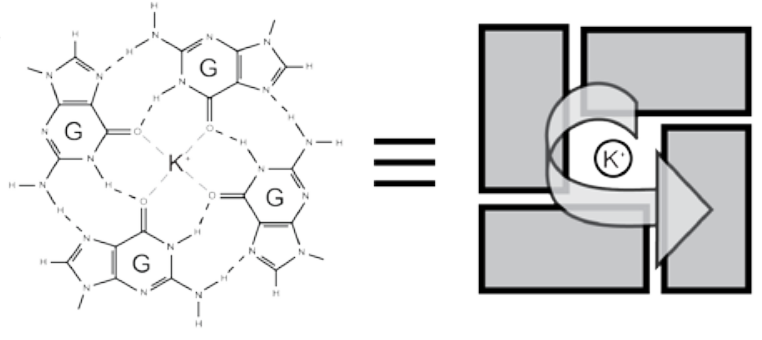

B

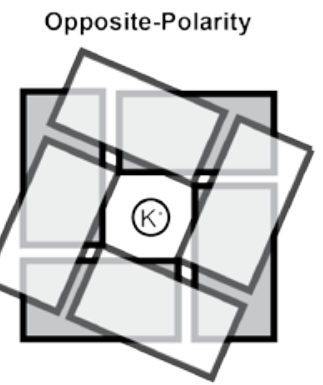

C
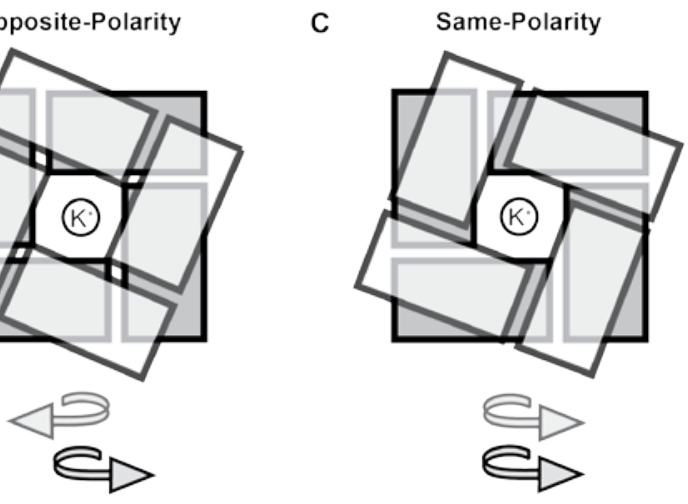

D

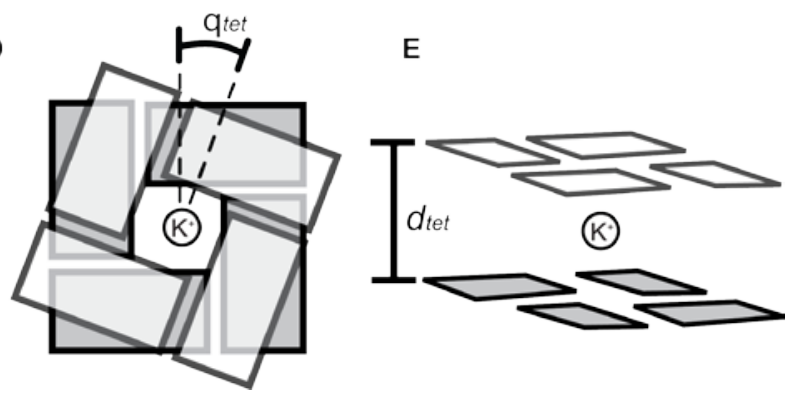

Figure 2-1. G-tetrad stacking types: (A) G-tetrads have a polarity as defined by direction of the Hoogsteen bond donor-to-acceptor pattern. Tetrad stacking can be primarily described as (B) opposite-polarity or (C) same-polarity stacking. Model stacked G-tetrad geometries used in this study varied by (D) relative rotation angle $\left(\theta_{t e t}\right)$ and (E) G-tetrad separation distance $\left(d_{t e t}\right)$.

druplexes. Molecular dynamics (MD) has been used in past work to investigate the energy differences of G-quadruplexes with varying structural features $(59,158,159)$. In this work we evaluate the ability of an AMBER molecular mechanics (MM) description of energy to accurately represent the base stacking energy of coordinated guanine-tetrads in comparison to QM calculations. MM computed energy differences between base stacking modes are discussed with special attention paid to their implications towards high-order G-quadruplex assemblies. MD simulations are carried out to investigate the observed preference in our survey of G-quadruplexes to stack predominantly in a 5'-5' 
manner, with the G-tetrad stacking interface occurring between the 5'-end of individual G-quadruplex units. MD simulations provide some explanation for this observation in terms of different accessible base stacking geometries between 3'-3' and 5'-5' stacked G-quadruplexes. 


\subsection{Nomenclature:}

The stacking of adjacent G-tetrads can be described by the relative polarity of the Hoogsteen hydrogen-bond pattern (Fig. 2-1). Within a single G-tetrad, the hydrogen-bond polarity is defined in the direction of hydrogen-bond donor to acceptor, from N2-H to N7 and from N1-H to O6. Two neighboring G-tetrads can therefore be described as belonging to one of two classes of stacking: 1) "same-polarity" if the polarities are in the same direction (Fig. 2-1C) or 2) "opposite-polarity” if in the opposite direction (Fig. 2-1B). The possible geometries of stacked guanine bases are fundamentally different between sameand opposite-polarity stacked G-tetrads. Nomenclature of commonly occurring base stacking modes from experiment are based upon a description of the aromatic-ring overlap of stacked guanines. For example, stacking geometry characterized by overlap of the 5-member rings of guanine bases is termed 5-ring stacking. Within the G-quadruplex core, the geometries of stacked guanines are related to glycosidic conformation of the bases (61) and are therefore sometimes referred to in these terms. For example, the Syn/Anti nomenclature describes the base stacking geometry of the 5'-Syn $\rightarrow$ Anti-3' dinucleotide step.

In this paper we refer to stacked guanine bases as belonging to one of two classes of stacking termed same-polarity and opposite-polarity. In literature, it is common for these classes to be further divided and referred to as head-to-tail or tail-to-head belonging to same-polarity stacking and head-to-head or tail-to-tail belonging to opposite-polarity stacking (160). In this notation, head and tail refer to the different faces of a guanine base. In the isolated planar G-tetrad models used in our QM studies there is no difference between head-to-head and tail-to-tail models or the head-to-tail and tail-to-head models of similar separation and relative rotation. For this reason, we classify stacking primarily as 
belonging to either the same-polarity or opposite-polarity type.

The G-tetrad models used for both energy calculations and structural characterization are symmetric planar guanine assemblies. We allow the relative rotation angle, termed $\theta_{\text {tet }}$ (Fig. 2-1D), and the separation distance $d_{\text {tet }}$ defined to be the distance between parallel planar tetrads (Fig. 2-1E), to vary in our models. Due to the nature of G-tetrads and the symmetry of our models, the geometries of stacked guanines repeat as $\theta_{\text {tet }}$ varies over $90^{\circ}$ intervals. In the case of opposite-polarity stacked tetrads, the $\theta_{\text {tet }}$ angle between stacked tetrads is defined to be the rotation of one tetrad, in the direction of its hydrogen-bond polarity, with respect to the other through the central axis perpendicular to the tetrad plane. In the case of same-polarity stacked tetrads, the $\theta_{\text {tet }}$ angle is defined arbitrarily to be the rotation of the tetrad containing the 5'-most guanine being considered in the direction of its hydrogen-bond polarity. The $\theta_{\text {tet }}$ angle is defined to be zero when $\mathrm{O} 6$ atoms of stacked tetrads are on top of each other such that the inter-tetrad O6-O6 distance of stacked guanines is at its minimum and is equal to $d_{t e t}$, the separation between G-tetrads.

The stacking of individual G-quadruplexes at a G-tetrad interface is most readily observed for parallel-stranded G-quadruplexes. This G-quadruplex folding topology gives rise to well-defined 3'- and 5'-ends as determined by the 5'-to-3' directionality of the strands in the G-tetrad core. The 5' -5 ' notation, for example, is used to describe the stacking of two individual G-quadruplexes at the tetrad interface on their 5'-ends. 


\subsection{Theory:}

The QM-computed interaction energy, termed “stacking energy” or $\Delta E$, represents the gas-phase energy difference between a complex of stacked $\mathrm{K}^{+}$-coordinated G-tetrads and isolated fragments as shown in eq. 1.

$$
\Delta E=E^{\text {complex }}-E^{\text {tetrad } 1}-E^{\text {tetrad 2 }}-E^{\text {ion }}
$$

The counterpoise procedure is used to correct for basis set superposition errors (161). Here, $E^{\text {complex }}$ is the total energy of the complex, while $E^{\text {tetrad1 }}, E^{\text {tetrad2 }}$ and $E^{\text {ion }}$ are the energies of individual tetrads and a central coordinating ion respectively. For QMcomputed energy landscapes, $\Delta E^{\text {norm }}$ represents the normalized stacking energy, determined relative to the minimum $\Delta E$ observed across both same- and opposite-polarity landscapes. For higher-level MP2/6-311+G(2d,2p) calculations, $\Delta E^{\text {norm }}$ was determined relative to the lowest $\Delta E$ across the geometries computed. For MM-computed energy landscapes, $E^{\text {Amber }}$ represents the total MM description of system energy for a given geometry, normalized relative to the minimum across both same- and opposite-polarity landscapes. Let us note how interaction energy differs from the free energy of a system. Interaction energy, or stacking energy, is the energy associated with molecules interacting in gas phase. This energy is calculated to describe how favorable the geometry of a molecular ensemble is compared to sum of each molecule in isolation. These calculations are limited to a quantum description of interactions in the absence of solvent. In fully represented and solvated systems, other interactions such as hydrophobic interactions and the polarization of solute molecules by solvent influence the total free energy of the system. 


\subsection{Results and Discussion:}

\subsection{1: Structural survey and characterization: Guanine stacking within the G-tetrad core}

A structural survey of the PDB was performed in order to characterize the guanine base stacking geometries observed between tetrads within the G-tetrad core of crystallographic G-quadruplex structures (Appendix III Table A3-1). Individual G-quadruplex units were found to have three common stacking geometries within the G-tetrad core (Fig. 2-2), which are intrinsically related to the glycosidic conformation of the bases: 1) Partial 5/6ring stacking (Fig. 2-2A) of the Anti/Anti step is formed from same-polarity stacked tetrads with a partial overlap of the 5-member ring of one guanine with the 6-member ring of another; 2) 5-ring stacking (Fig. 2-2B) of the Syn/Anti step is a result of oppositepolarity stacking with an overlap of the 5-member rings of stacked guanines; 3) Partial 6ring stacking (Fig. 2-2C) of the Anti/Syn step is also formed by opposite-polarity stacked tetrads and exhibits a partial overlap of the 6-member rings of stacked guanines.

To quantify the stacking geometries of different core guanine-guanine steps, stacked gua-

A

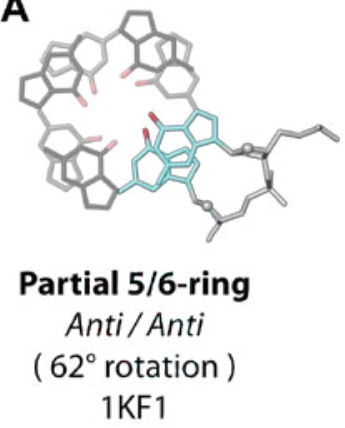

B

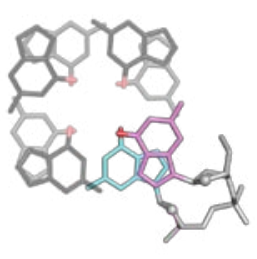

5-ring

Syn / Anti

$\left(84^{\circ}\right.$ rotation )

$1 \mathrm{JPQ}$

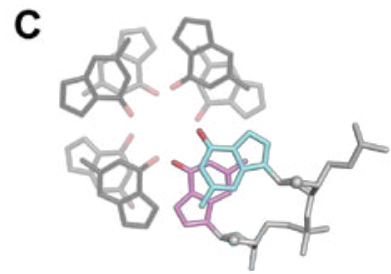

Partial 6-ring

Anti/Syn

$\left(28^{\circ}\right.$ rotation )

$1 \mathrm{JPQ}$

Figure 2-2. Illustrative examples of core base stacking modes from the structural survey. (A) 'Partial 5/6ring' stacking of the 'Anti/Anti' step, (B) '5-ring' stacking of the 'Syn/Anti' step and (C) 'Partial 6-ring stacking' of the 'Anti/Syn' as observed in crystallographic structures. 'Syn' and 'Anti' glycosidic conformations are depicted as magenta and cyan, respectively. Stacking modes are labeled along with the $\theta_{\text {tet }}$ rotation value and the PDB ID code of the experimental structure from which the geometries are taken. 
nines from the tetrads of experimentally determined structures were compared to a library of stacked guanines from model G-tetrads varying over relative rotation $\left(\theta_{\text {tet }}\right)$ and separation $\left(d_{t e t}\right)$ space (Fig. 2-1). Values of these parameters were assigned to experimental pairs of stacked guanines via lowest RMSD alignment with model tetrads (See Appendix II - Protocols - Chapter 2 - Fig A2-1). The results of our characterization of experimental guanine base stacking geometries are shown in Figure 2-3 and Table 2-1. Expectedly, there exist discrete regions of $\theta_{\text {tet }}$ for the Anti/Anti, Anti/Syn and Syn/Anti steps (Fig. 2-3A). Unexpectedly, the average $d_{\text {tet }}$ was found to vary among stacking modes (Fig. 2-3B and Table 2-1). Average $d_{\text {tet }}$ values of 3.39, 3.47, and $3.55 \AA$ observed
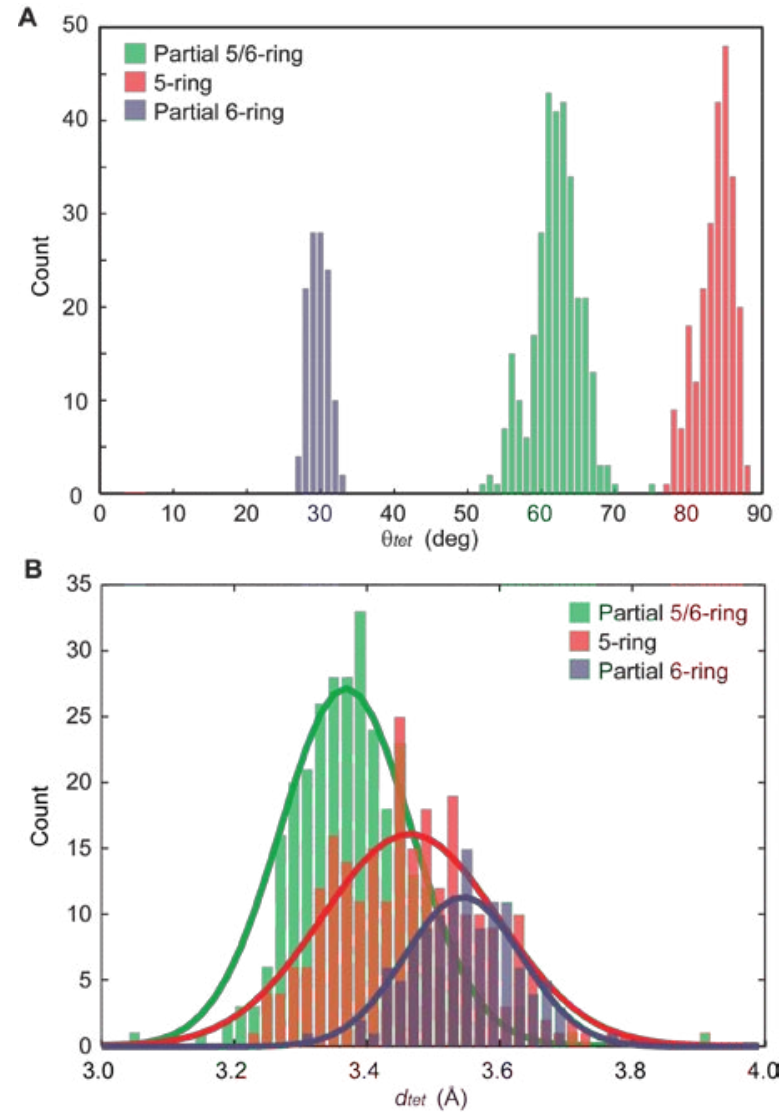

Figure 2-3: Histograms representing the (A) relative rotation $\left(\theta_{\text {tet }}\right)$ and (B) separation $\left(d_{t e t}\right)$ values observed for stacked guanines within the Gquadruplex core of cataloged crystallographic G-quadruplexes. Counts are binned every $1^{\circ}\left(\theta_{\text {tet }}\right)$ and $0.02 \AA\left(d_{t e t}\right)$. Separation histograms are fitted with Gaussian distributions for visualization purposes. For a detailed explanation of how geometrical parameters are extracted from experimental structures see Appendix II - Protocols - Chapter 2. 
Table 2-1. Statistics of base stacking modes as observed in experimentally determined structures.

\begin{tabular}{|c|c|c|c|c|c|c|c|c|c|c|}
\hline \multirow{3}{*}{ Stacking Mode } & \multicolumn{5}{|c|}{ X-ray } & \multicolumn{5}{|c|}{$\mathrm{NMR}^{\mathrm{b}}$} \\
\hline & \multirow{2}{*}{ Count $^{a}$} & \multicolumn{2}{|c|}{$\theta_{\text {tet }}\left({ }^{\circ}\right)$} & \multicolumn{2}{|c|}{$d_{\text {tet }}(\AA)$} & \multirow{2}{*}{ Count $^{\mathrm{C}}$} & \multicolumn{2}{|c|}{$\theta_{\text {tet }}\left({ }^{\circ}\right)$} & \multicolumn{2}{|c|}{$d_{\text {tet }}(\AA)$} \\
\hline & & Avg & $1^{\text {st }}$ s.d. $^{\text {d }}$ & Avg & $1^{\text {st }}$ s.d. $^{\text {d }}$ & & Avg & $1^{\text {st }}$ s.d. $^{\text {d }}$ & Avg & $1^{\text {st }}$ s.d. ${ }^{\mathrm{d}}$ \\
\hline \multicolumn{11}{|l|}{ Core } \\
\hline $\begin{array}{l}\text { Partial 5/6-ring } \\
\text { (Anti / Anti) }\end{array}$ & 310 & 61.5 & 3.3 & 3.39 & 0.10 & 23 & 61.1 & 5.5 & 3.28 & 0.17 \\
\hline $\begin{array}{c}\text { 5-ring } \\
\text { (Syn / Anti) }\end{array}$ & 245 & 83.2 & 2.4 & 3.47 & 0.11 & 44 & 84.5 & 4.7 & 3.54 & 0.29 \\
\hline $\begin{array}{l}\text { Partial 6-ring } \\
\text { (Anti / Syn) }\end{array}$ & 118 & 29.2 & 1.4 & 3.55 & 0.08 & 8 & 30.7 & 8.2 & 3.57 & 0.29 \\
\hline \multicolumn{11}{|l|}{ Interface } \\
\hline Partial 6-ring & 4 & 26.2 & - & 3.50 & - & - & - & - & - & - \\
\hline 6-ring & 4 & 41.6 & - & 3.41 & - & - & - & - & - & - \\
\hline 5/6-ring & 3 & 56.5 & - & 3.41 & - & $15^{\mathrm{e}}$ & 61.8 & - & 3.43 & - \\
\hline 5-ring & 33 & 83.2 & 1.4 & 3.52 & 0.08 & $5^{e}$ & 81.4 & - & 3.11 & - \\
\hline \multicolumn{11}{|c|}{$\begin{array}{l}{ }^{a} \text { The number of observed base stacking geometries that are defined uniquely within the unit cell or within the interface of symmetry } \\
\text { mates. } \\
\text { b A selection of available NMR structures (Appendix III - Table A3-2) were cataloged in order to determine if there exist fundamental } \\
\text { differences between the base stacking modes exhibited in X-ray and NMR structures. } \\
\text { c For NMR structures, the base stacking geometry of a single pair of stacked guanines is an average across all of the models present in } \\
\text { a given PDB file. } \\
\text { d Standard deviations values highlight the ensemble spread of our characterized geometries used to determine } \theta_{\text {tet }} \text { and } d_{\text {tet }} \text {. They do not } \\
\text { reflect the uncertainty in experimental measurements. } \\
\text { e the interface geometries of some NMR structures (PDB I.D. 1MY9 and 2RQJ) were observed to contain mixed base stacking modes } \\
\text { at their stacking interface. }\end{array}$} \\
\hline
\end{tabular}

for the Anti/Anti, Syn/Anti and Anti/Syn steps respectively. However, regions of $d_{\text {tet }}$ (Fig.

3B) for different base stacking modes are highly overlapped.

In order to account for a possible bias of experimental conditions on our statistical results of base stacking geometries, sub-classes of G-quadruplex structures were also analyzed. When appropriate, crystallographic structures were further classified by their varying structural features (Table 2-2), including whether the oligonucleotide chemistry was DNA or RNA, the type of coordinating ion, the presence of a drug molecule, strand directionality, and strand stoichiometry. No significant differences are observed among the features investigated in this work. To see if our statistical findings were specific to crystallographic structures, a set of NMR structures were also examined (Table 2-1 and Appendix III - Table A3-2). Similar average values of $d_{\text {tet }}$ and $\theta_{\text {tet }}$ were observed for the 
Table 2-2. Sub-classification of stacked guanine geometries within the G-tetrad core of cataloged crystallographic G-quadruplex structures

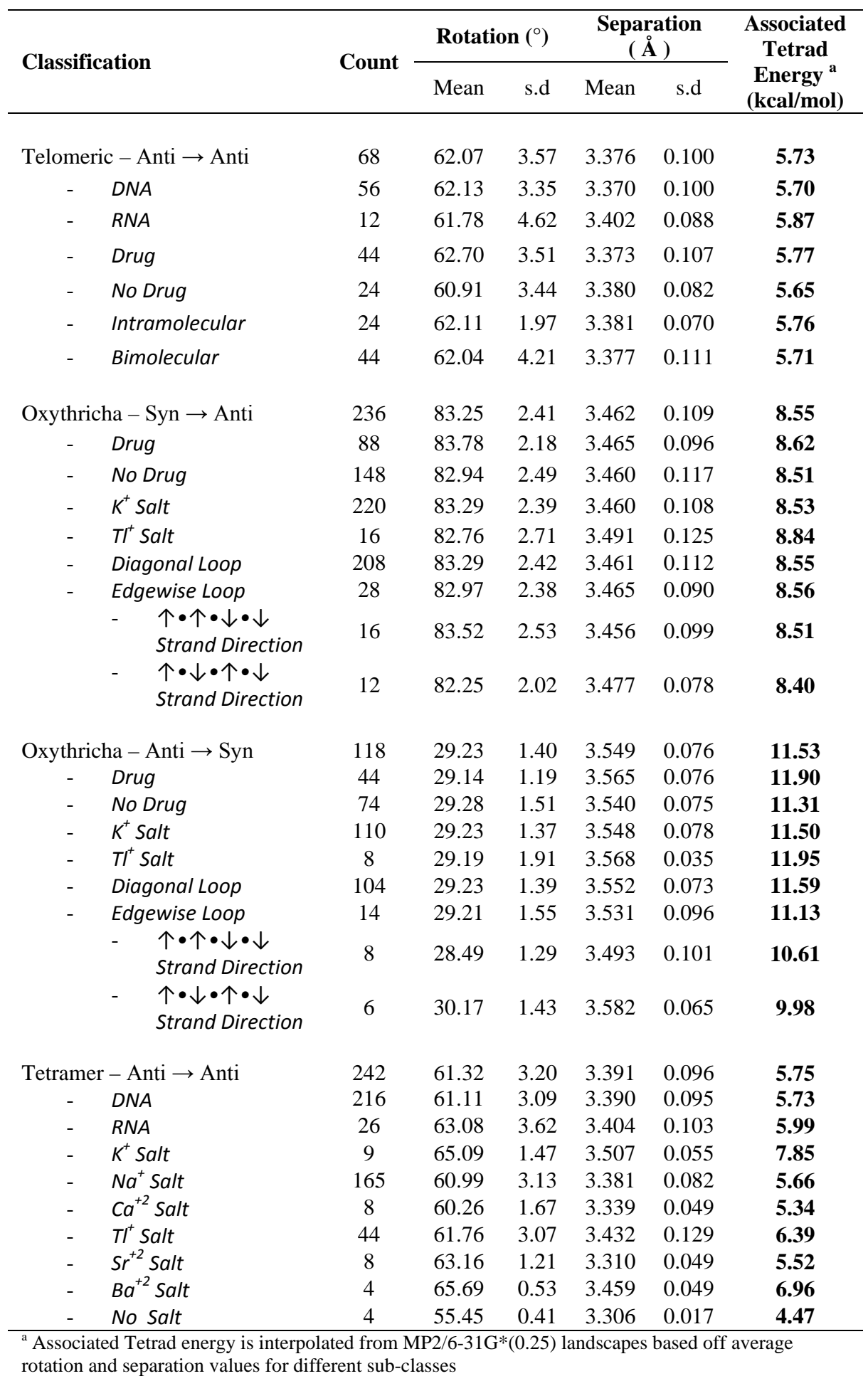

Anti/Anti, Syn/Anti, and Anti/Syn core stacking modes. It is important to state that there was a notable occurrence of Anti/Syn geometries surveyed from crystal structures in which the stacked guanines were non-parallel, containing varying pitch angles about the 
C2-C8 axis of an individual guanine. While our study is limited to understanding the effects of $\theta_{\text {tet }}$ and $d_{\text {tet }}$ on stacking energy, it would be interesting to study the effects of other degrees of freedom on stacked coordinated G-tetrads.

The statistical information in Table 2-1 offers insight into the differences between core guanine base stacking geometries. While different average $\theta_{\text {tet }}$ angles are fundamental to unique stacking modes, perhaps unintuitive is the observed differences in average $d_{\text {tet }}$ of stacked guanines. Another feature of note is that the variation in the $1^{\text {st }}$ standard deviation of $\theta_{\text {tet }}$ with Anti/Anti $>$ Syn/Anti $>$ Anti/Syn. Notably, stacked guanines within the core of NMR structures demonstrate much larger $1^{\text {st }}$ standard deviation values suggesting the geometries of stacked core guanines are more variable in NMR solution structures compared to crystallographic structures. This variation may reflect the different nature of crystal and solution environments or limitations of NMR-based models in providing the atomic precision necessary for a rigorous discussion of base stacking geometry. Considering the limitations in accuracy of experimentally determined structures, we must be cautious in our interpretation of statistical results dealing with small structural differences such as the average $d_{t e t}$ values for core stacking modes.

To better interpret the statistical data of Table 2-1, let us discuss the structural motifs included in this study. The Anti/Anti step is abundant in crystallographic structures of Gquadruplex telomeric sequences $(21,64,150,156,162,163)$ containing the $\mathrm{T}_{2} \mathrm{AG}_{3}$ motif and its variants, as well as structures of tetrameric G-quadruplexes (7,62,145-149,164-168). The Syn/Anti and Anti/Syn steps are abundant in G-quadruplexes formed from Oxytricha telomeric sequences (169-174) containing the $\mathrm{G}_{4} \mathrm{~T}_{4} \mathrm{G}_{4}$ motif and its variants. Additionally, Syn/Anti steps are observed in some bulged RNA tetramer G-quadruplexes $(165,175)$. The Syn/Syn stacking mode is observed in some NMR structures of unique folding topologies 
(28,61,176-178). Similar to Anti/Anti steps, Syn/Syn steps also exhibit a Partial-5/6ring stacking geometry. The Syn/Syn step is not discussed here in length, as it has not been observed in crystallographic structures of G-quadruplex which to date are less diverse than NMR structures in terms of sequence and folding topology.

\subsection{2: Structural survey and characterization: Guanine stacking at the G-tetrad interface of stacked G-quadruplexes}

In our survey of the PDB we also cataloged the base stacking geometries at the interface of stacked G-quadruplexes. The G-tetrad stacking interfaces of stacked G-quadruplex arise due to dimerization of individual G-quadruplex units in solution or in a crystal packing environment. We include both crystallographic and NMR structures in our survey due to the limited number of stacked G-quadruplex complexes published to date. Experimentally observed interface geometries may be classified into four modes (Fig. 24). These stacking geometries are termed Partial 6-ring (Fig. 2-4A), 6-ring (Fig. 2-4B), 5/6-ring (Fig. 2-4C) and 5-ring (Fig. 2-4D) based on the description of aromatic ring overlap observed between pairs of stacked guanine bases. We note that all interface stacking exhibits opposite-polarity stacking. Additionally, almost all parallel Gquadruplexes that exhibit interface stacking do so at their 5'-end. The preference for 5'-5' interface stacking is examined further in subsequent sections.

The 6-ring, 5/6-ring, and 5-ring stacking modes are observed at the interfaces of crystallographic tetrameric (7,62,145-149) and human telomeric (21,64) G-quadruplex structures (Appendix III - Table A3-1 and A3-2). A single instance of Partial 6-ring stacking is observed in an G-quadruplex structure formed by the sequence $d\left[\mathrm{G}_{4}{ }^{\mathrm{Br}} \mathrm{UT}_{2} \mathrm{G}_{4}\right]_{2}$ demonstrating $2+2(\uparrow \cdot \uparrow \cdot \downarrow \cdot \downarrow)$ strand directionality (63). Interface stacked G-tetrad geomet- 
A

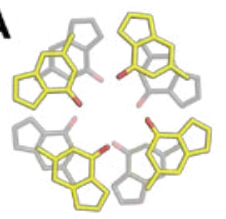

Partial 6-Ring

( $26^{\circ}$ rotation )

2AVJ
B

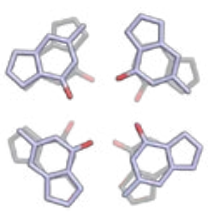

6-Ring

( $46^{\circ}$ rotation ) $2 \mathrm{HRI}$
C

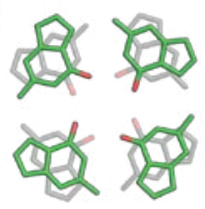

5/6-Ring

( $56^{\circ}$ rotation )

$1 \mathrm{KF} 1$
D

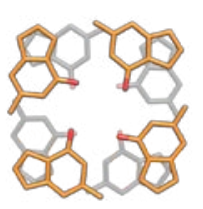

5-Ring

( $82^{\circ}$ rotation )

$352 \mathrm{D}$

Figure 2-4. Illustrative examples of G-tetrad base stacking modes at the interface of stacked Gquadruplexes as identified in our structural survey. (A) 'Partial 6-ring', (B) '6-ring', (C) '5/6-ring' and (D) '5-ring' as observed in crystallographic structures. Stacking modes are labeled along with the $\theta_{\text {tet }}$ rotational value and the PDB ID code of the experimental structure from which the G-tetrads are taken.

ries in crystallographic structures exhibited mostly 5-ring stacking (Table 2-1), largely due to the high number tetrameric structures formed from the $d\left[\mathrm{TG}_{4} \mathrm{~T}\right]$ sequence which predominantly demonstrated 5-ring stacking (Fig. 2-4D).

NMR structures containing G-quadruplex interfaces were mostly propeller-type and demonstrated either the 5/6-ring or the 5-ring stacking geometries (Fig. 2-C,D). It is hard to draw any conclusions regarding different interface stacking preferences of NMR and crystallographic structures due to the differences in the types of G-quadruplexes studied. Interface base stacking geometries are sometimes found within interlocked Gquadruplexes such as the Partial 6-ring stacking of the interlocked V4 folding topology (65). Interlocked structures $(65,74,75)$ are dimeric G-quadruplexes without a freestacking interface, consisting of two stacked G-quadruplex units each contributing guanines to G-tetrad core of the other. These unique geometries are not considered in our statistics. 


\subsection{3: QM computational studies: Energy landscapes of $\mathrm{K}+$ coordinated stacked G-tetrads}

We employed a series of QM computations to calculate stacking energies (see section 2.2: Nomenclature), of many stacked G-tetrad geometries coordinated by a centralized $\mathrm{K}^{+}$ion. The $\theta_{\text {tet }}$ and $d_{\text {tet }}$ values of stacked tetrads were systematically varied to generate energy landscapes of same-polarity and opposite-polarity stacked tetrads at the MP2/6$31 G^{*}(0.25)$ level (Fig. 2-5). Slices of energy landscapes at a $d_{\text {tet }}$ value of $3.3 \AA$ illustrates the $\theta_{\text {tet }}$ energy dependence of same- and opposite-polarity stacked tetrads (Fig. 2-5A).

The $\theta_{\text {tet }}$ energy profile of same-polarity stacking is characterized by highly unfavorable st acking in the area of low $\theta_{\text {tet }}\left(<15^{\circ}\right.$ and $\left.>75^{\circ}\right)$ and a broad energy minimum elsewhere $\left(15^{\circ}-75^{\circ}\right)$ with a global minimum at $45^{\circ}$ and two shoulders of comparable energy at $15^{\circ}$ and $75^{\circ}$ separated by a small energy barrier of roughly $2.5 \mathrm{kcal} / \mathrm{mol}$. The energy landscape of the same-polarity stacked tetrad is presented (Fig. 2-5B), superimposed with regions indicating average geometries of base stacking modes cataloged from crystal structures (Table 2-1). There exists a broad low-energy basin within the $d_{\text {tet }}$ range of 3.2 $\AA$ to $3.4 \AA$ and $15^{\circ}$ to $75^{\circ}$. The Anti/Anti core stacking geometry (Fig. 2-2A) is observed to fall well within this energy basin (Fig. 2-5B) in close proximity to the landscapes low energy states. The broad low energy basin of the same-polarity energy landscape may in part explain the relatively large $1^{\text {st }} \mathrm{SD}$ of $\theta_{\text {tet }}$ exhibited by the Anti/Anti same-polarity stacking mode (Table 2-1). It is interesting to note that the most favorable same-polarity stacking occurs at rotation $45^{\circ}$ when there is little aromatic ring overlap of stacked bases.

In contrast, the $\theta_{\text {tet }}$ energy profile of opposite-polarity stacking is characterized by a deep global minimum around $55^{\circ}-60^{\circ}$ and local minima at $35^{\circ}-45^{\circ}$ and $85^{\circ}-5^{\circ}$ of approximate- 

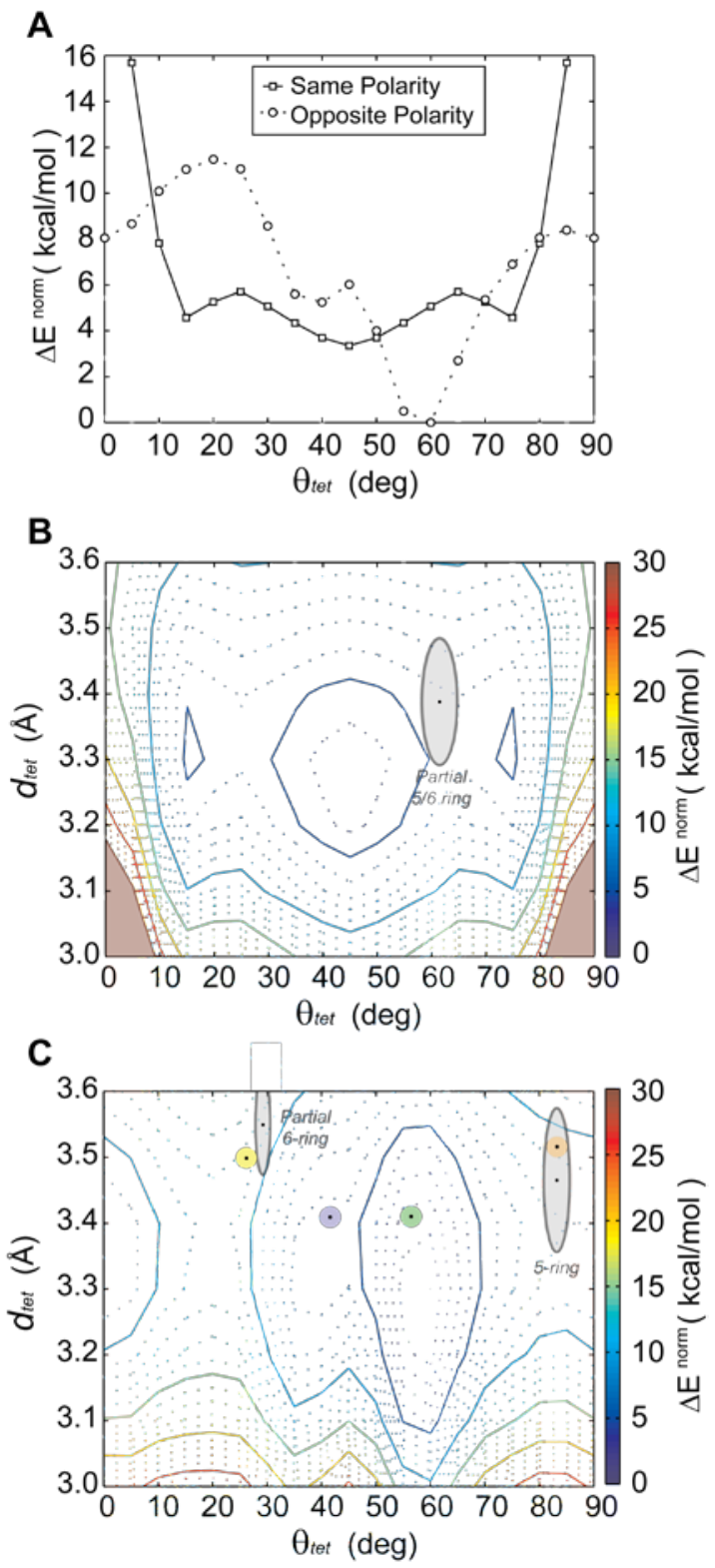

Figure 2-5: QM energy landscapes. (A) The rotational $\left(\theta_{t e t}\right)$ energy profile of same-polarity and opposite-polarity stacked G-tetrads at a $d_{t e t}$ value of $3.3 \mathrm{~A}^{\circ}$ separation. Energy landscapes of (B) same-polarity and (C) opposite-polarity stacked tetrads are presented with stacking modes as determined from cataloged crystallographic structures superimposed. Average core G-tetrad stacking modes are shown with average geometries indicated by a black dot and gray outlined regions having dimensions of the first SD for $\theta_{\text {tet }}$ and $d_{\text {tet }}$ values. Average G-tetrad stacking modes at the interface of stacked G-quadruplexes are presented, color-coded as shown in Figure 2-4: 'Partial 6-ring' (yellow), ‘6-ring' (purple), ‘5/6-ring’ (green) and ‘5-ring’ (orange).

ly 6 and $8 \mathrm{kcal} / \mathrm{mol}$ respectively (Fig. 2-5A). There is a sizable energy barrier of 4 $\mathrm{kcal} / \mathrm{mol}$ separating the $85^{\circ}-5^{\circ}$ and $35^{\circ}-45^{\circ}$ minima. The energy landscape of oppositepolarity stacked tetrads (Fig. 2-5C) is a diverse landscape characterized by narrow energy 
minima. The Anti/Syn Partial 6-ring and Syn/Anti 5-ring core stacking modes as well as interface stacking modes can be seen superimposed upon the opposite-polarity stacking landscape (Fig. 2-5C). Interestingly, the core G-tetrad stacking modes do not fall within calculated minima. Alternatively, the 5/6-ring interface stacking mode occupies the global minimum $\left(55^{\circ}-60^{\circ}\right)$ with the 6-ring interface stacking mode being found in the neighboring energy minimum $\left(35^{\circ}-45^{\circ}\right)$. It is very interesting that tetrad stacking at the interface of stacked G-quadruplexes, which is not constrained by a backbone, more readily occupies the energy minima of the QM computed energy landscapes. We note that the most favorable interface stacking geometry, the 5/6-ring mode, is found in both crystallographic and NMR structures.

The relative stacking energies of average G-tetrad stacking geometries from experimentally determined structures were interpolated from the energy landscape and are presented in Table 2-3. Additionally, stacking energies were computed at a higher level of theory, MP2/6-311+G(2d,2p), for stacked G-tetrads that mimic the average experimental stacking geometries observed in our survey (Table 2-3). $\Delta E^{\text {norm }}$ stacking energies of core

Table 2-3. Calculated tetrad stacking energies (kcal/mol) of experimentally observed stacking modes

\begin{tabular}{|c|c|c|c|c|c|}
\hline \multirow{2}{*}{ Stacking Mode } & \multicolumn{2}{|c|}{ MP2 $6-31 G^{*}(0.25)^{\mathrm{a}}$} & \multicolumn{2}{|c|}{ MP2 $6-311+G(2 d, 2 p)$} & \multirow{2}{*}{$\begin{array}{c}\text { AMBER } \\
E^{\text {Amber }}\end{array}$} \\
\hline & $\Delta \mathrm{E}$ & $\Delta \mathrm{E}^{\text {Norm }}$ & $\Delta \mathrm{E}$ & $\Delta \mathrm{E}^{\text {Norm }}$ & \\
\hline \multicolumn{6}{|l|}{ Core } \\
\hline Partial 5/6-ring & -153.56 & 5.74 & -157.37 & 4.21 & 2.07 \\
\hline 5-ring & -150.74 & 8.56 & -153.77 & 7.81 & 3.64 \\
\hline Partial 6-ring & -147.71 & 11.59 & -149.70 & 11.88 & 10.09 \\
\hline \multicolumn{6}{|l|}{ Interface } \\
\hline Partial 6-ring & -147.78 & 11.52 & -149.90 & 11.69 & 9.24 \\
\hline 6-ring & -153.72 & 5.58 & -156.71 & 4.88 & 5.27 \\
\hline 5/6-ring & -157.66 & 1.64 & -161.58 & 0 & 2.29 \\
\hline 5-ring & -149.99 & 9.31 & -152.45 & 9.14 & 4.76 \\
\hline
\end{tabular}


tetrad stacking geometries were found to be ranked Anti/Anti Partial 5/6-ring < Syn/Anti 5-ring < Anti/Syn Partial 6-ring with values of 4.21, 7.81 and $11.88 \mathrm{kcal} / \mathrm{mol}$ respectively. For interface tetrad stacking geometries, $\Delta E^{\text {norm }}$ is ranked as follows: 5/6ring $<$ 6-ring $<$ 5-ring $<$ Partial 6-ring with values of 0.00, 4.88, 9.14 and $11.69 \mathrm{kcal} / \mathrm{mol}$ respectively. The stacking energies interpolated from MP2/6-31G*(0.25) calculations of average experimental geometries are in agreement with higher level MP2/6$311+G(2 d, 2 p)$ calculations (Table 2-3). It is important to remember that relative energies discussed here are for entire stacked tetrads, not a single pair of stacked guanines.

Since a large number of cataloged structures contain $\mathrm{Na}^{+}$ions instead of $\mathrm{K}^{+}$ions, the effects of $\mathrm{Na}^{+}$on the energy profiles of stacked tetrads was also investigated (Fig 2-6). Models were run with a central $\mathrm{K}^{+}$, a central $\mathrm{Na}^{+}$, two $\mathrm{Na}^{+}$ions in the tetrad plane, and no ion present. The rotational energy profiles for all of these models are very similar. However, ion type does affect the separation energy profile. When $\mathrm{K}^{+}$is substituted with $\mathrm{Na}^{+}$, the energy minimum is lowered. The use of two $\mathrm{Na}^{+}$ions is more similar to the curve of $\mathrm{K}^{+}$, except slightly flattened. Interestingly, the use of two $\mathrm{Na}^{+}$ions, one in the center of each tetrad, leads to an energy profile that is almost identical to model with no coordinating ion. In the presented $\mathrm{Na}^{+}$-containing crystal structures, ions are found in a range between the two extremes explored in these models with the $2 \mathrm{Na}^{+}$motif being slightly more observed. With this in mind we include all structures in the statistics used to derive relative experimental base stacking energies (Table 2-3).

One should use caution when comparing quantitative relative energies from gas phase en ergy calculations of isolated tetrads with experimentally observed tetrad stacking geometries. For reasons discussed above (see Section 2.3: Theory), the energy dependen- 


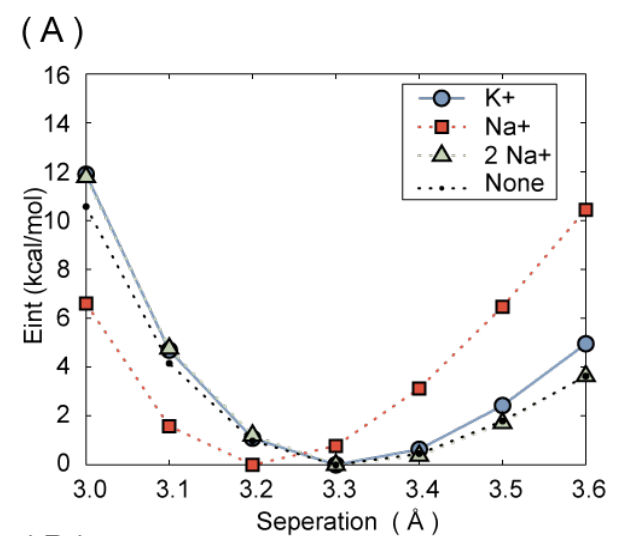

(B)

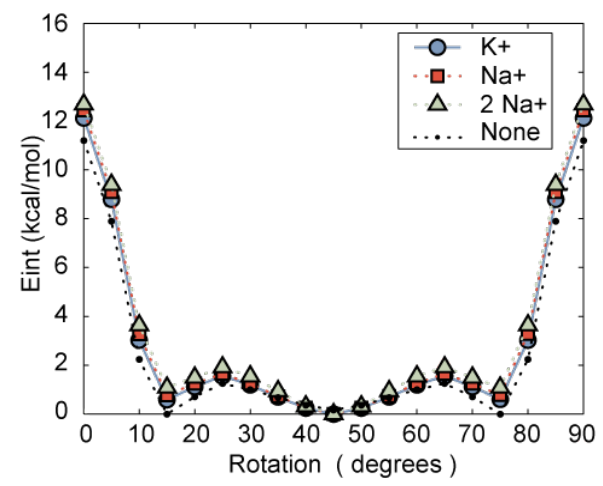

( C )

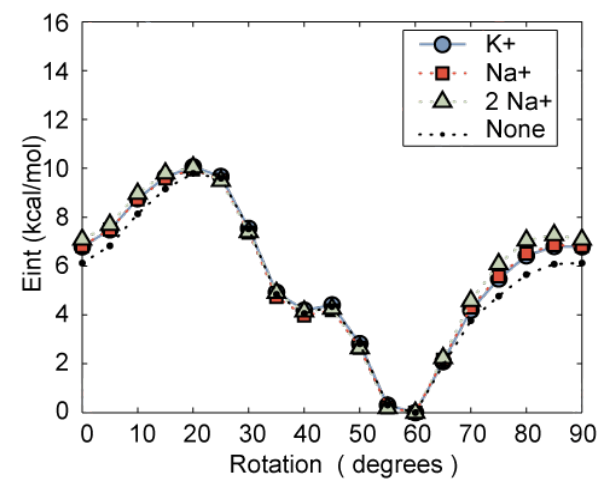

Figure 2-6: Effect of ion type on QM energy profiles of stacked Gtetrads containing central $\mathrm{K}^{+}(\mathrm{O})$, central $\mathrm{Na}^{+}(\square)$, and two $\mathrm{Na}^{+}$, one in the center of each tetrad $(\Delta)$ : (A) Separation profile of $60^{\circ}$ samepolarity stacked tetrads. Similar energy profiles are observed for the $\mathrm{K}^{+}$ and two- $\mathrm{Na}^{+}$containing models. (B) Rotational energy profile of samepolarity stacked tetrads at $3.4 \AA$ separation. (C) Rotational energy profile of opposite-polarity stacked tetrads at $3.4 \AA$ separation. Energy profiles have been individually normalized with their individual minima Interaction energy taken to be zero.

ce of tetrad geometries calculated in gas phase can be altered by a water environment. For example, interactions of tetrad edges with ions and water are energetic contributions that can differ with varying tetrad geometries $(150,156,162,163)$. When considering the accuracy of experimental structures, atomic coordinate precision can be on the order of 
the geometrical differences examined in computational models. For these reason, we only compare QM calculations with results from our survey of crystallographic structures. These structures are expected to be generally better resolved than NMR. Despite these uncertainties, the results of our structural survey of crystallographic structures, particularly interface stacking geometries, are in good agreement with our computational studies suggesting that computed energy landscapes provide a good general description of preferable G-tetrad stacking geometries in G-quadruplex systems. Results from fully represented and solvated MD simulations of G-quadruplexes (discuss below) further support these findings.

Previous quantum chemical studies of G-tetrad stacking have also examined some of the base stacking geometries discussed in this study (142-144). A study by Gu et al. (143) in 2002 examined two geometries of stacked G-tetrad complexes with different coordinated cations. Initial geometries were similar to those found in crystallographic structures and then optimized at the Hartree-Fock (HF) level of theory. Optimized tetrad geometries of 5/6-ring stacking were observed for an opposite-polarity model and an optimized $\theta_{\text {tet }}$ of $\sim 45^{\circ}$ observed for a same polarity model. While far from their starting structures geometries, optimized tetrads in this study are in agreement with the two global minima observed in our study. In 2005, Meyer et al. (144) examined such tetrads in a similar manner using Density Functional Theory (DFT). Three optimized geometries were obtained based on model tetrads of different symmetries. These included the two geometries discussed above and a third corresponding to the global maximum of our same polarity landscape with a $\theta_{\text {tet }}$ of $\sim 0^{\circ}$. These past studies have examined G-tetrad stacking geometries for same- and opposite-polarity stacked tetrads and give an idea of the energy differences between them. The current study expands on previous works by a 
systematic assessment of stacking energy landscapes at a high level of theory, MP2/6$31 G^{*}(0.25)$, and couples this investigation with a structural survey of experimental Gtetrad stacking geometries.

\subsection{4: Molecular Mechanics energy landscapes of stacked G-tetrads}

In addition to our QM investigation of G-tetrad stacking, we decided to assess the accuracy of a commonly used molecular mechanics (MM) force field in reproducing QM G-tetrad stacking landscapes. Knowledge of how well G-tetrad stacking interactions are represented in MD simulations is important to the structural determination and modeling G-quadruplexes.

Energy landscapes were computed for $\mathrm{K}^{+}$coordinated stacked G-tetrads using the ff94 AMBER force field (179) description of intermolecular Van der waals and electrostatic terms which have remain unchanged among commonly used revisions to this force field for nucleic acid simulations. The results of these studies are shown in Figure 2-7. The shape of the MM same-polarity energy landscape (Fig 2-7B) is very similar to that of the QM landscape, with a similar broad energy basin observed. The low $\theta_{\text {tet }}$ regions of the same-polarity energy landscape are again observed to be unfavorable. The oppositepolarity stacking landscape computed via the AMBER force field (Fig. 2-7C) is also generally similar in shape to that of the QM landscape except with smaller and less defined energy barriers. More specifically, the energy barriers between the 5/6-ring and 5ring as well as the Partial 6-ring and the 6-ring base stacking modes are practically negligible.

The differences between the QM and the MM energy landscapes lead to some notable dif- 

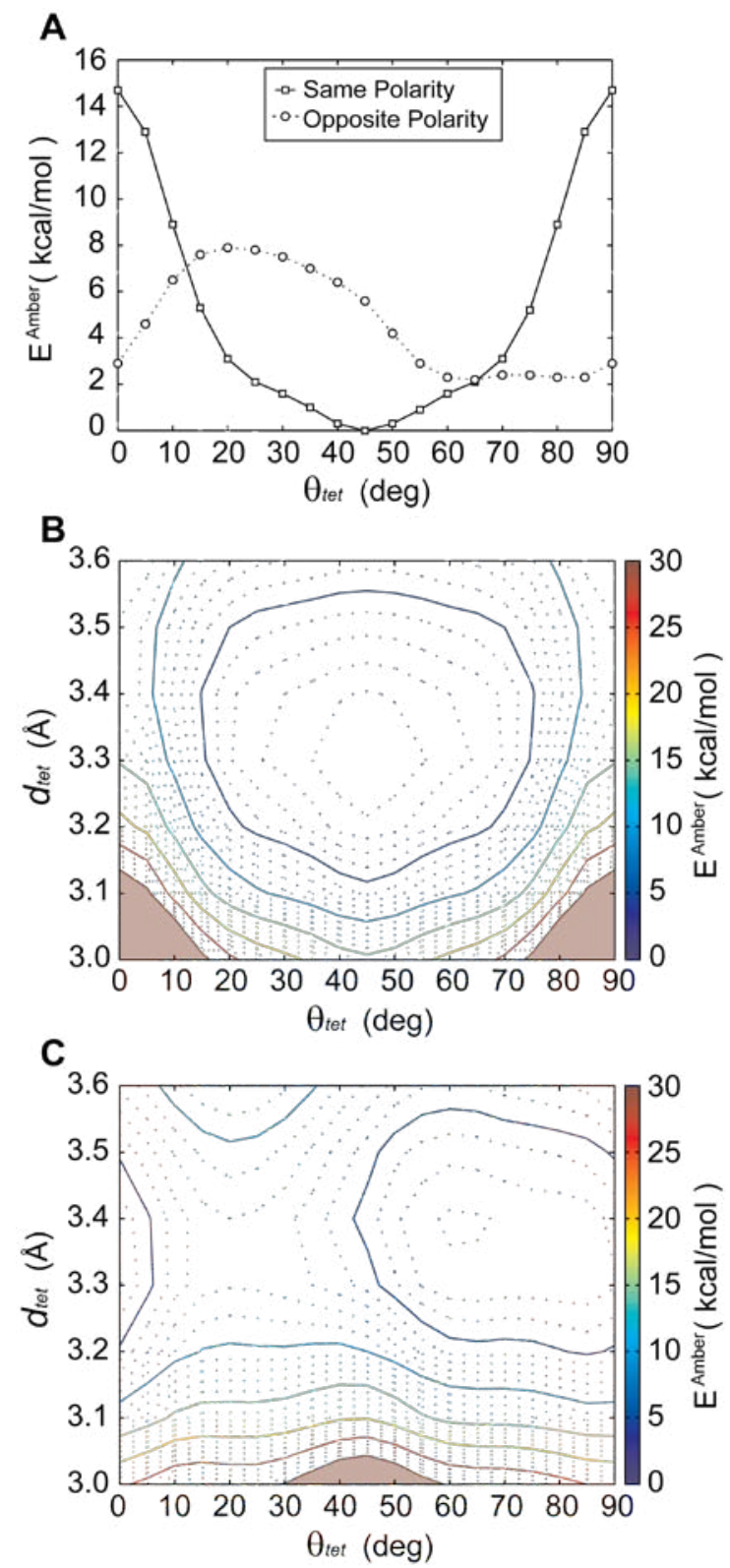

Figure 2-7: MM energy landscapes. (A) Rotational energy profile of same-polarity and opposite-polarity stacked tetrads at a $d_{\text {tet }}$ value of $3.3 \AA$ separation. (B) MM energy landscapes of same-polarity and (C) opposite-polarity stacked tetrads.

ferences in the relative energies of various stacking modes (Table 2-3), particularly for the Partial 5/6-ring and 5-ring modes of the core and the 5/6-ring and 5-ring modes of the interface. The less-defined energy minima of the opposite-polarity energy landscape computed via AMBER should be considered when using such techniques in modeling Gquadruplex structures. Generally speaking however, the AMBER force field shows a good agreement with QM computations, and favors the 5/6-ring and 5-ring Interface stacking 
modes, which are seen occupied by all NMR-based experimental structures and most crystallographic structures. This agreement is interesting considering that the description of intermolecular interactions in the AMBER force field is limited to Van der Waals and electrostatic interactions.

Recently, studies have employed free energy analysis of MD simulations to explore the effects of varying structural features on the energy of G-quadruplex systems $(59,159)$. Studies by Cang et al. examined energy differences of different di-nucleotide steps in the context of G-quadruplex DNA (59). A difference of $15 \mathrm{kcal} / \mathrm{mol}$, calculated using MMPBSA free energy analysis of MD simulations, is observed between 2-layered Gquadruplexes containing all Syn/Anti steps and one containing less favorable Anti/Anti steps. Our ranking of Anti/Anti Partial 5/6-ring $<$ Syn/Anti 5-ring $<$ Anti/Syn Partial 6ring for core base stacking geometries does not directly contrast these results, as our work seeks to understand the energy contribution of base stacking which is only one component driving the formation of G-quadruplex structures. In a fully represented and solvated system, it is apparent that many other factors play a role. It is interesting to note that a comparison of QM and MM energy landscapes suggest a relative energy difference of 3.4 and $1.6 \mathrm{kcal} / \mathrm{mol}$, respectively, between the more favorable Anti/Anti Partial 5/6-ring and the Syn/Anti 5-ring stacking geometries suggesting some discrepancy between the QM computed landscapes and the MM description of G-tetrad stacking energy. Nevertheless, the MM energy landscapes reproduce the general trends observed in QM energy landscapes suggesting a fairly accurate description of the relative stacking energy between stacked G-tetrad geometries. 


\subsection{5: Investigating 3'-3' and 5'-5' G-quadruplex stacking using Molecular Dynamics}

Individual G-quadruplex blocks are sometimes observed to stack upon each other at the interface of their outermost exposed G-tetrads. Parallel-stranded G-quadruplexes contain well defined 3'- and 5'-ends giving rise to four possible stacking combinations (180), 5'5', 3'-3', 5'-3' and 3'-5'. Stacking that is 5' $-5^{\prime}$ ' or 3'-3' arises from opposite-polarity tetrad stacking, while 5'-3' and 3'-5' arises from same-polarity tetrad stacking. No instances of 5'-3'stacking at the G-tetrad interface of G-quadruplexes have been reported, although unpublished data from our laboratory suggest that 5'-3' stacking can occur at the interface of two G-quadruplexes connected by a linker. In our survey, G-quadruplex stacking interfaces are almost entirely observed to be 5'-5' in nature. In tetramer Gquadruplex forming sequences, stacking is observed in a 5'-5' manner despite the presence of 3' and 5' flanking ends for most sequences $(7,62,145,146,148,149)$. Of sequences with well defined free stacking interfaces, the only exception observed to stack in 3'-3' manner is a bimolecular telomeric G-quadruplex whose 5' end is occupied by small-molecule binding (64). NMR studies of the 5'-TTAGGG sequence demonstrate that when the 5'-end of a tetrameric G-quadruplex is occupied by a sizable flanking sequence, 3'-3' stacking still readily occurs (181). These studies suggest that 3'-3' stacking is possible, but less favorable compared to the more readily observed 5'-5' stacking. It is also interesting to note that some instances have been observed of drugmediated 5’-5’ stacking (163).

In order to shed light on the observed 5'-5' stacking preference, a series of MD simulations was performed. Using a variant from an NMR based model (182), we simulated the stacking of two propeller structures formed by the sequence $d\left[\mathrm{G}_{3}\left(\mathrm{TG}_{3}\right)_{3}\right]$. 
Individual G-quadruplex units were manually oriented at their $3{ }^{\prime}-3^{\prime}$ or 5 ' -5 ' ends in multiple starting geometries. The $\theta_{\text {tet }}$ of interface stacked guanines was determined along the trajectories in order to understand the base stacking modes occupied by 3'-3' and 5'5’ stacked G-quadruplexes (Fig. 2-8A,B).

For all trajectories, the $\theta_{\text {tet }}$ values of tetrads at the interface of the stacked propeller Gquadruplexes equilibrate within the first few nanoseconds of simulation. More interestingly, each of the three trajectories run for the 3'-3' and 5'-5' models equilibrate to a single $\theta_{\text {tet }}$ value, regardless of their initial $\theta_{\text {tet }}$ value. What is perhaps most interesting is that the $3^{\prime}-3^{\prime}$ and $5^{\prime}-5^{\prime}$ stacking models equilibrate to very different interface geometries. The interface geometries of the 3'-3' model equilibrate to $\theta_{\text {tet }}$ value of roughly $40^{\circ}-45^{\circ}$ corresponding to the 6-ring stacking mode (Fig. 2-4B), as seen experimentally (147), while the $5^{\prime}-5^{\prime}$ ' model equilibrates to $60^{\circ}-65^{\circ}$ similar to $5 / 6$-ring stacking (Fig. 2-4C). Longer MD simulations of 100 ns were computed for select trajectories to investigate if these stacking geometries are maintained over a longer time scale (Fig. 2-8C). Both the 3'-3' and the 5'-5' models remain folded and stacked throughout the 100 ns trajectories. The 3'-3' stacked G-quadruplexes are observed to periodically shift from the more favorable of 6-ring stacking to a Partial 6-ring stacking geometry with a $\theta_{\text {tet }}$ of roughly $30^{\circ}$. The $5^{\prime}-5^{\prime}$ 'model remains in an equilibrium interface geometry exhibiting 5/6-ring stacking over the entire 100 ns trajectory.

Structural analysis suggests that the 3'-3' model cannot occupy the interfacial 5/6-ring stacking mode, as this would result in a clash between the sugars of the stacked guanines (Fig. 2-9). Conversely, the 5/6-ring stacking of the 5'-5' model is easily achieved without unfavorable backbone interactions (Fig. 2-9A). Calculations at the MP2/6-311+G(2d,2p) 
A

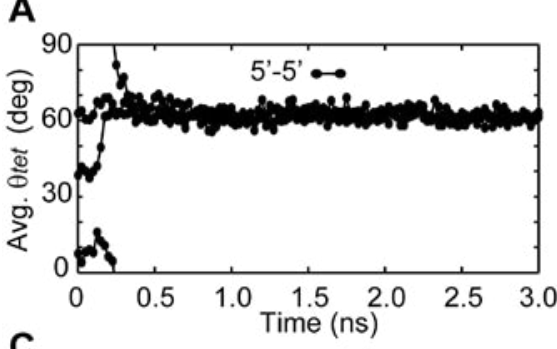

B

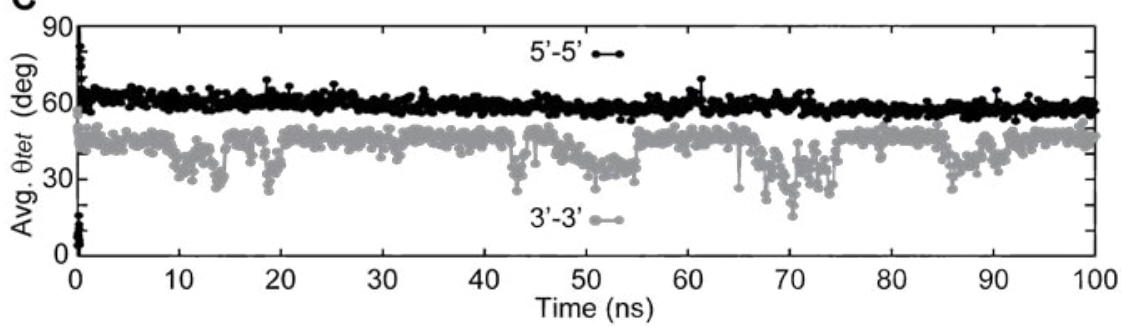

Figure 2-8. MD investigation of G-quadruplex G-tetrad stacking interfaces: 5-ns trajectories of stacked propeller G-quadruplexes formed by the sequences $d\left[\left(G_{3} T\right)_{3} G_{3}\right]$ were run for (A) 5'-5' and (B) 3'-3' stacked G-quadruplexes. The trajectories of three different starting geometries of G-quadruplex stacking arrangements (Interface $\theta_{\text {tet }}$ varying by $\sim 30^{\circ}$ ) are shown. (C) Long 100-ns trajectories were computed for a 50-50 and a 30-30 stacked G-quadruplex complex. Average relative rotation $\left(\theta_{t e t}\right)$ of the four interface guanine stacks was characterized at regular intervals.

level described in Table 2-3 suggest that the 6-ring and 5/6-ring stacking modes are energetically different by roughly $5 \mathrm{kcal} / \mathrm{mol}$. Based on the QM and MD studies described in this work, we provide evidence that accessible base stacking modes are fundamentally different for 3'-3' and 5'-5' stacked G-quadruplexes. Furthermore, we suggest that sizable energetic differences between accessible base stacking modes play a role in the observed preference for G-quadruplexes to stack primarily in a 5'-5' manner.

Understanding the relative energies of accessible interfacial base stacking modes has important implications in high-order stacking of G-quadruplexes. Past studies have suggested that long telomeric repeats form a "bead-on-a-string" model in which long telomeric nucleic acids form a series of intramolecular quadruplex "beads" $(48,183)$. Other studies $(21,155,180,184)$ suggest that further structural organization is possible, in which individual beads stack upon each other on the 3'-end and/or the 5'-end G-tetrad interfaces. Stacking models have been suggested in which telomeric G-quadruplex beads 

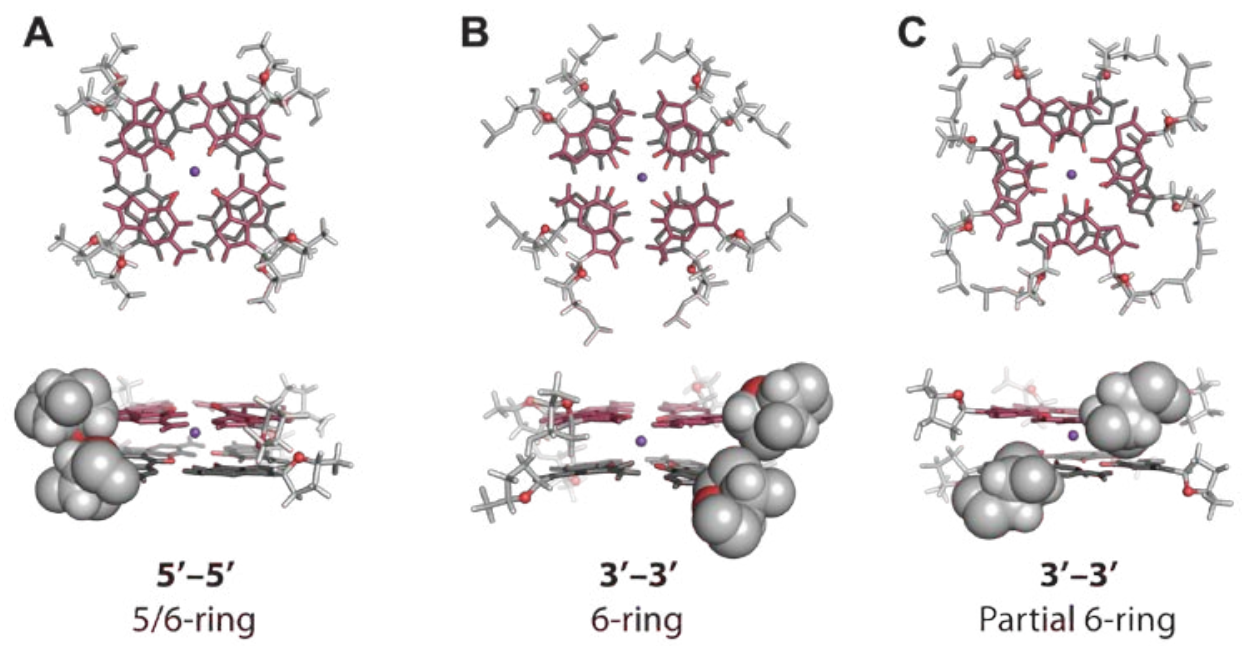

Figure 2-9: Illustrative interface geometry of MD trajectories: (A) A '5/6-ring' stacking equilibrium is maintained across the 100-ns trajectory for the 5'-5' stacking model. (B) The interface of the 3'-3' model is mostly found in a '6-ring' stacking geometry. (C) Occasionally the 3'-3' model occupies a 'Partial 6-ring' stacking mode. Backbone atoms are shown in light gray and the O1' atoms of sugar are shown in red. Spherical representation of backbone atoms are the approximate Van der Waals surface of atoms as visualized in PyMOL.

stack in a 5'-3' (21,184), 3'-3' (155), and 5'-5' manner (155,180). Crystallographic structural data has shown drug-mediated 5'-3' stacking interactions in 2-repeat human telomeric DNA (162). Additionally, 5'-5' stacking has been observed in 2-repeat (150) and 4-repeat (21) human telomeric DNA. NMR solution studies have observed 5'-5' Gtetrad stacking in 2-repeat human telomeric RNA (155). Additionally, recent studies have suggested that TERRA stacks preferentially in a 2-block manner $(180,185)$. This observation is in line with a $5^{\prime}-5^{\prime}$ or $3^{\prime}-3^{\prime}$ model as $5^{\prime}-3^{\prime}$ stacking is expected to demonstrate continuous stacking beyond two blocks. The preference of 5'-5' stacking observed in our survey supports 5'-5' stacking models of higher-ordered telomeric repeat structures and are in agreement with recent studies by Martadinata et al. (180) which report a 5/6-ring interface stacking geometry of the 5'-5' stacked TERRA based on experimental and MD studies. While it is probable that multiple types of high-order Gquadruplex assemblies can form in dynamic long telomeric nucleic acids, further work 
Chapter 2: Base Stacking and High-Order Assemblies

needs to be done to understand which are most readily adopted in the context of biologically relevant environments. 


\subsection{Conclusions:}

The current study seeks to understand the roles of guanine base stacking in the formation and interaction of G-quadruplex nucleic acids. We undertake an extensive survey of the PDB to characterize guanine base stacking geometries within the core and at the interface of G-quadruplexes. We couple this survey with systematic QM and MM computations of G-tetrad stacking energy landscapes, helping to understand the base stacking energy contributions for different G-tetrad geometries observed experimentally. We also perform MD simulations to investigate the roles of base stacking in high-order G-quadruplex assemblies. We conclude the following from our studies:

1) There exist three well-defined base stacking modes within the G-tetrad core of Gquadruplexes as well as numerous commonly occurring stacked G-tetrad geometries at the interface of stacked G-quadruplex structures. Base stacking geometries within the core were unique for the Anti/Anti, Syn/Anti, and Anti/Syn di-nucleotides steps. Additional base stacking geometries were observed at the interface, with the 5-ring and 5/6-ring stacking modes being most readily observed.

2) In our survey of the PDB, G-tetrad stacking at the interface of G-quadruplexes in both NMR and crystallographic structures always displayed opposite-polarity stacking and were primarily stacked in a 5' -5 ' manner.

3) QM energy landscapes of $\mathrm{K}^{+}$-coordinated stacked G-tetrads were calculated for different stacking polarities. Tetrad stacking energy for experimental geometries are ranked as follows, core stacking modes: Anti/Anti Partial 5/6-ring < Syn/Anti 5-ring < Anti/Syn Partial 6-ring, interface stacking modes: 5/6-ring $<$ 6-ring $<5$ ring < Partial 6-ring. G-tetrad stacking geometries at the interface of Gquadruplex units are found to be energetically different in terms of stacking 
energy by up to $12 \mathrm{kcal} / \mathrm{mol}$.

4) Aside a few small differences, MM computed G-tetrad stacking energy landscapes are in good agreement with QM landscapes suggesting that relative G-tetrad stacking energies are well represented in MD simulations.

5) MD simulations suggest that 5'-5' and 3'-3' stacking of parallel-stranded Gquadruplexes have different accessible base stacking modes, with 5'-5' stacking being more energetically stable in terms of tetrad stacking energy. The results of our survey and computational studies suggest that a 5'-5' arrangement with the 5/6-ring base stacking geometry of the interfacial G-tetrads is a favorable arrangement of stacked parallel G-quadruplexes. 


\title{
Chapter 3:
}

\section{Fundamentals of G-quadruplexes: Charge Transport}

\begin{abstract}
This chapter was largely adapted from the authors work: Lech, C.J., Phan, A.T., Michel-Beyerle, M., Voityuk, A.A. (2013) Electron-Hole Transfer in G-quadruplexes with Different Tetrad Stacking Geometries: A Combined QM and MD Study. Journal of Physical Chemistry B, $117,9851-9856$

Author Contributions: MD Simulations and analysis of MD data was performed by C.J.L.; Geometrical characterization of G-tetrad stacks was done by A.A.V.; QM computations were performed by A.A.V.; Computational approaches were designed by A.A.V. and C.J.L.; QM data analysis was performed by C.J.L and A.A.V.; All authors contributed to the experimental conceptualization and writing of above the manuscript from which this chapter is based.
\end{abstract}

\subsection{Introduction:}

The ability for DNA to host long range charge transport has important implications in both biology and nanotechnology. Charge transport enables the relocation of DNA damage into particular areas of the genome and may play a role in protein signaling (186). From a nanotechnology perspective, the growing need for nanoscale electronics has produced a rising interest in DNA as a potential element for building nanoscale circuitry. Previous studies have demonstrated a wide range of electron hole-transfer (HT) properties of DNA duplexes and related systems (187-190). In particular, 10 years ago, it was shown that the efficiency of HT in DNA strongly depends on conformational fluctuations $(191,192)$. Subsequently, this finding was supported by many other studies (See references $(189,190)$ and references therein). A deeper understanding of the relationship between kinetics and structure is expected to help to improve the design of DNA based 
molecular wires (193-196).

As an alternative to double helical structures, four-stranded G-quadruplex nucleic acids represent an attractive biopolymer for the formation of conductive nanoscale wires (Gwires) (91,92,197-199). G-quadruplexes are stabilized by the stacking of Hoogsteenbound guanine tetrads (G-tetrads) and have a propensity to form in ionic solution, or, perhaps better, in aqueous electrolytes (200). Stacked G-tetrads were reported to be efficient electron traps that may function in vivo as electron sinks, diverting oxidative damage away from other genomic regions (201). There are only a few studies focusing on electron-transport properties of G-quadruplexes (202-205). While cation coordinated Gtetrads of G-quadruplexes have more $\pi$-contacts along the stack compared to doublestranded DNA, the relative conductive properties of these two classes of structures is still unclear $(201,202,205,206)$.

Computational studies of nucleic acids reveal many important details of the structure and dynamics of systems that are difficult to derive from experimental data (207). Both quantum mechanics (QM) and molecular dynamics (MD) approaches have provided valuable insights into G-tetrad stacking geometries and interaction energies $(59,71,143,158,208)$. Electronic structure calculations have shown that electronic states in G-quadruplexes are more delocalized than in DNA duplexes (209,210). Recently, the electrical conduction properties of a tetrameric parallel-stranded G-quadruplex were thoroughly investigated using a QM/MD approach (211).

Within G-quadruplex nucleic acids, the guanine base stacking geometry of stacked Gtetrads can vary greatly depending on the conformation adopted (67). Several unique G- 
tetrad stacking modes can arise from different folding patterns within a single Gquadruplex molecule or at the interface of two stacked G-quadruplex subunits (212). It is currently unclear how the orientation of stacked G-tetrads affects the mobility of electron holes through the G-tetrad stack.

In this study, we address this question by examining how the probability of HT varies with G-tetrad stacking geometry. Through a combined QM and MD study of Gquadruplex systems (Appendix II - Protocols - Chapter 3), we demonstrate that HT rates within the G-tetrad stacks vary greatly for different commonly occurring stacking geometries. By identifying experimentally observable G-tetrad stacking geometries with optimal HT properties, our findings reveal important structural considerations for the design of conductive nanowires built upon the G-quadruplex architecture. 


\subsection{Theory:}

\subsubsection{Electronic Coupling}

The well established Fragment Charge Difference (FCD) method $(213,214)$ was employed to derive the quantities $V$ from the adiabatic electronic states of the G-tetrad stacks.

In the two-site model of the FCD method, we consider the transfer of an electron hole from a donor " $D$ " to an acceptor " $A$ " site. The system can be described in terms of the two orthogonal and normalized states, $\Psi_{1}$ and $\Psi_{2}$, with corresponding energies $\mathrm{E}_{1}$ and $\mathrm{E}_{2}$ :

$$
\begin{aligned}
& \Psi_{1}=\mathrm{c}_{\mathrm{D} 1} \Phi_{\mathrm{D}}^{\tilde{\mathrm{N}}}+\mathrm{c}_{\mathrm{A} 1} \Phi_{\mathrm{A}}^{\tilde{\mathrm{A}}} \\
& \Psi_{2}=\mathrm{c}_{\mathrm{D} 2} \Phi_{\mathrm{D}}^{\tilde{\mathrm{D}}}+\mathrm{c}_{\mathrm{A} 2} \Phi_{\mathrm{A}}^{\tilde{A}}
\end{aligned}
$$

Where the functions $\Phi_{\mathrm{D}}^{\sim}$ and $\Phi_{\mathrm{A}}^{\sim}$ correspond to normalized wave functions that are localized on the donor and acceptor sites. The charge on the donor or acceptor fragment, $\mathrm{F}=(\mathrm{D}, \mathrm{A})$, in the state $\Psi_{\mathrm{i}}, \mathrm{i}=(1,2)$, is simply $\mathrm{c}_{\mathrm{Fi}}^{2}$. By applying the following orthogonal transformation T:

$$
T=\left(\begin{array}{cc}
\cos (\omega) & -\sin (\omega) \\
\sin (\omega) & \cos (\omega)
\end{array}\right)
$$

to the states $\Psi_{1}$ and $\Psi_{2}$, we can obtain two new states described by:

$$
\begin{aligned}
& \Phi_{\mathrm{D}}=\Psi_{1} \cos (\omega)+\Psi_{2} \sin (\omega) \\
& \Phi_{\mathrm{A}}=-\Psi_{1} \sin (\omega)+\Psi_{2} \cos (\omega)
\end{aligned}
$$

The charge localized on the donor, $q(D)$, in the states $\Phi_{\mathrm{D}}$ and $\Phi_{\mathrm{A}}$ can be expressed as: 


$$
\begin{aligned}
q(D) & =\left(c_{D 1} \cos (\omega)+c_{D 2} \sin (\omega)\right)^{2} \\
& =q_{1}(D) \cos ^{2}(\omega)+q_{2}(D) \sin ^{2}(\omega)+q_{12}(D) \sin (2 \omega) \\
q(A) & =\left(c_{A 2} \cos (\omega)-c_{A 1} \sin (\omega)\right)^{2} \\
& =q_{2}(A) \cos ^{2}(\omega)+q_{1}(A) \sin ^{2}(\omega)-q_{12}(A) \sin (2 \omega)
\end{aligned}
$$

where $\mathrm{q}_{12}(\mathrm{~F})=\mathrm{C}_{\mathrm{F} 1} \mathrm{C}_{\mathrm{F} 2}$. By maximizing the value $\mathrm{q}(\mathrm{D})+\mathrm{q}(\mathrm{A})$ we determine the value of $\omega$ that corresponds to maximum charge transfer between the donor and acceptor, leading to:

$$
\tan 2 \omega=\frac{2 \Delta q_{12}}{\Delta q_{1}-\Delta q_{2}}
$$

where $\Delta q_{12}=\Delta q_{12}(D)-\Delta q_{12}(A)$. Considering a two-state model, the electronic coupling between donor and acceptor can be written as:

$$
V=\frac{1}{2}\left(E_{2}-E_{1}\right) \sin 2 \omega
$$

By combining Eq. 6 and Eq. 7, the electronic coupling matrix element between the donor site $D$ and the acceptor site $A$ can be written as:

$$
V=\frac{\left(E_{2}-E_{1}\right) \Delta q_{12}}{\sqrt{\left(\Delta q_{1}-\Delta q_{2}\right)^{2}+4 \Delta q_{12}^{2}}}
$$

Here $\Delta q_{1}$ and $\Delta q_{2}$ are the donor-acceptor charge differences in the adiabatic states with energies $E_{1}$ and $E_{2}$, and $\Delta q_{12}$ is the corresponding off-diagonal term.

These quantities were calculated within the self-consistent field approximation using the semi-empirical INDO/S method (215). The method was shown to be computationally very efficient, offering surprisingly good estimates for electronic couplings in DNA stacks (216). These advantages enable quantum mechanical treatment of several thousand 
different conformations of stacked G-tetrads.

\subsubsection{Hole Transfer Rates}

The non-adiabatic electron transfer rate $k_{\mathrm{ET}}$ can be expressed as (217):

$$
k_{E T}=\frac{2 \pi}{\hbar} V^{2} F C
$$

where the electronic coupling $V$ is a key parameter that determines the sensitivity of the Electron transfer (ET) rate on the arrangement of the donor and acceptor sites. FC is the nuclear Frank-Condon factor that can be estimated as:

$$
F C=\frac{1}{\sqrt{4 \pi \lambda k_{B} T}} e^{\frac{-(\Delta G+\lambda)^{2}}{4 \lambda k_{B} T}}
$$

where $\Delta G$ is the free energy of ET and $\lambda$ is the reorganization energy. In the considered systems, the hole donor and acceptor sites are very similar and we assume that on average $\Delta G \approx 0$. In related DNA systems, the reorganization energy estimated with different approaches for HT between nucleobases ranges from 0.5 to1.5 eV (218-221). Due to the exponential dependence of $F C$ on $\lambda$, the estimated ET rate is extremely sensitive to the reorganization energy value used in Eq. (2) and decreases by a factor of $2 \cdot 10^{4}$ passing from $\lambda=0.5$ to $\lambda=1.5 \mathrm{eV}$. In this study, we are primarily interested in relative rates rather than in the absolute values. Assuming a similar reorganization energy and $F C$ for all stacks considered, we obtain that a relative rate value that depends only on the square of effective electronic coupling,

$$
k^{r e l}(X)=\frac{V^{2}(X)}{V^{2}(0)}
$$

where $V^{2}(0)$ corresponds to the reference system with an ET rate set equal to 1 . We 
computed the effective coupling for each ensemble of G-tetrad stacking geometries $V^{2}(X)$ (where $\mathrm{X}$ represents a given geometry of Fig 3-2A-E) by averaging of the matrix elements squared over $N$ structures $(N=1000)$ extracted from MD trajectories.

$$
V^{2}(X)=\overline{V^{2}}(X)=\frac{1}{N} \sum_{i=1}^{N} V_{i}^{2}(X)
$$




\subsection{Results and Discussion:}

\subsubsection{Geometry of Stacked G-tetrads}

G-tetrad stacking geometry can be simply classified by the relative polarity of stacked Gtetrads (Fig. 3-1A) The stacking of G-tetrads in a Same-Polarity or Opposite-Polarity manner defines the relative geometries that may be adopted by stacked guanines within G-tetrad stacks. Stacked G-tetrads are found within the core of a single G-quadruplex unit and at the stacking interface of dimeric G-quadruplexes and higher-order G-quadruplex assemblies. The geometry of stacked G-tetrads within a G-quadruplex or at the interface of stacked G-quadruplexes is highly sequence and structure dependent.

To investigate the electronic properties of stacked G-tetrads, we undertake QM calculations on G-tetrad stacks obtained from a series of MD simulations of G-quadruplex molecules (Fig 3-1B and Appendix II - Protocols - Chapter 3). A total of 1000 G-tetrad stacks are derived from MD data for each of the 5 unique G-tetrad stacking modes (Fig 3-

A
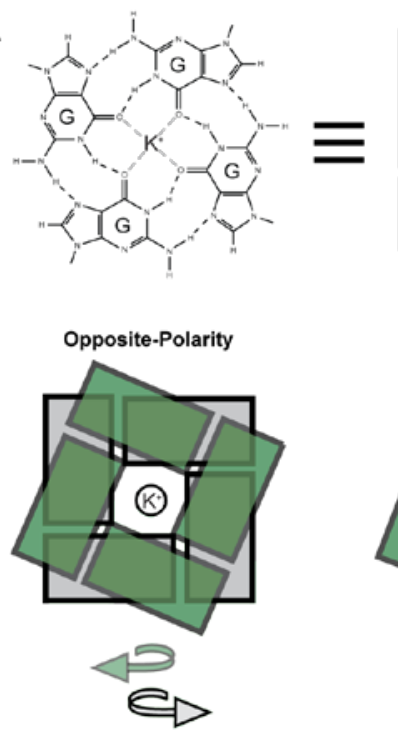
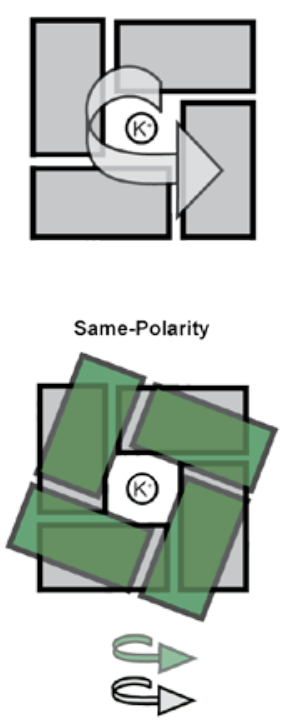

B

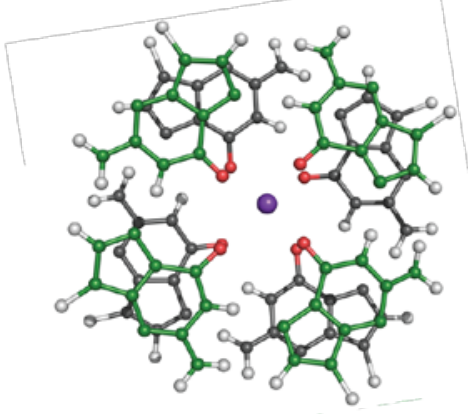

C

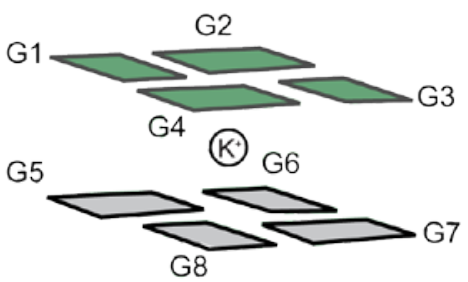

Figure 3-1. (A) Stacked G-tetrads are in either an Opposite-Polarity or Same-Polarity orientation. (B) Illustrative example of a stacked G-tetrad system with coordinating $\mathrm{K}^{+}$ion (purple) extracted from MD simulations. The $\mathrm{C}$ and $\mathrm{N}$ atoms of the guanine are colored by tetrad. The $\mathrm{H}, \mathrm{O}$, and $\mathrm{K}$ atoms are colored by type. (C) Relative position of guanines in the G-tetrad system referenced in Tables 3-2 and 3-4. 


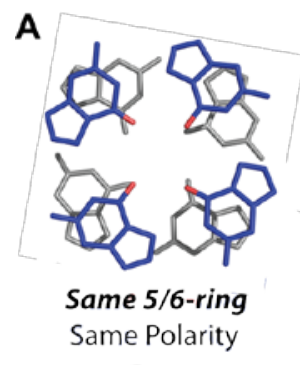

Core

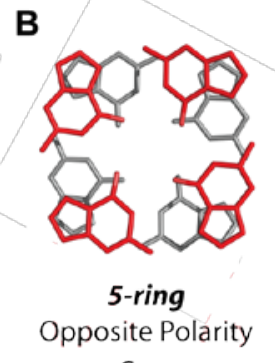

Core

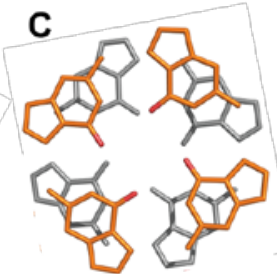

Partial 6-ring

Opposite Polarity

Core

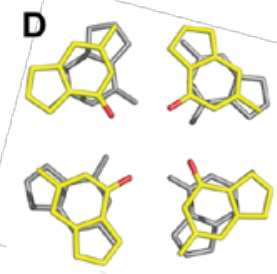

6-ring

Opposite Polarity

Interface

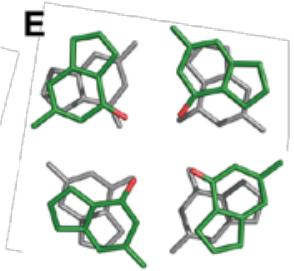

5/6-ring

Opposite Polarity Interface

Figure 3-2. Illustrative examples of G-tetrad stacking modes studied in this work

2). G-tetrad stacking geometries that commonly occur within the "core” of a single Gquadruplex unit were investigated (Fig 3-2A-C). We also examined “interface” stacking geometries that are observed when two G-quadruplex molecules dimerize at a G-tetrad interface (Fig 3-2D-E).

The Partial 5/6-ring G-tetrad stacking mode is a commonly occurring geometry in which the 6-member ring of one guanine base is partially overlapped with the 5-member ring of the other (Fig 3-2A). It is found within the G-tetrad core of a single G-quadruplex unit containing a parallel-stranded configuration. The 5-ring and Partial 6-ring geometries are named after the aromatic overlap of their rings, and are found within a single Gquadruplex unit containing a non parallel-stranded configuration (Fig. 3-2B-C). The 6ring and 5/6-ring geometries are more heavily overlapped geometries which occur at the interface of stacked G-quadruplex units (Fig. 3-2D-E).

The geometrical parameters of stacked G-tetrads were computed to explore the geometries sampled by the various stacking modes described in Figure 3-2. The data presented in Table 3-1 reports the average geometrical parameters and their standard deviations for the simulated G-tetrad systems considered in this chapter. 
Table 3-1. Average geometrical parameters of stacked G-tetrads and their standard deviations

\begin{tabular}{|c|c|c|c|c|}
\hline Stacking Orientation ${ }^{a}$ & Twist $^{\mathrm{b}}\left({ }^{\circ}\right)$ & $\operatorname{Rise}^{c}(\AA)$ & Inclination $^{\mathrm{d}}\left({ }^{\circ}\right)$ & Planar Shift ${ }^{\mathrm{e}}(\AA)$ \\
\hline Partial 5/6-ring (A) & $32.56 \pm 1.93$ & $3.43 \pm 0.06$ & $1.23 \pm 0.70$ & $0.22 \pm 0.11$ \\
\hline 5-ring (B) & $11.82 \pm 1.23$ & $3.49 \pm 0.07$ & $1.33 \pm 0.74$ & $0.26 \pm 0.13$ \\
\hline Partial 6-ring (C) & $43.42 \pm 1.20$ & $3.54 \pm 0.08$ & $1.66 \pm 0.95$ & $0.24 \pm 0.12$ \\
\hline 6-ring (D) & $27.10 \pm 1.99$ & $3.60 \pm 0.08$ & $2.19 \pm 1.11$ & $0.39 \pm 0.21$ \\
\hline 5/6-ring (E) & $-8.73 \pm 2.45$ & $3.37 \pm 0.07$ & $1.27 \pm 0.67$ & $0.31 \pm 0.16$ \\
\hline
\end{tabular}

The "Twist" parameter describes the relative rotation between two stacked tetrads. Variation in this parameter is a main feature of different G-tetrad stacking modes. It is important to note that the Partial 5/6-ring geometry arises from stacked G-tetrads of the same polarity. The other four stacking modes studied arise from G-tetrads of the opposite polarity. Relative values of "twist” are only comparable for G-tetrad stacks of similar stacking type (i.e. same polarity or opposite polarity).

The rise parameter represents the distance between G-tetrad planes. Values of this parameter are found to be within the range of 3.4 to $3.6 \AA$, as expected for $\pi$-stacked systems. Additionally, the variation in values of "rise" for different G-tetrad stacking geometries is in good agreement with our experimental survey (Chapter 2) in which we characterize this parameter for individual guanine stacks.

The "inclination" parameter measures the relative angle away from a parallel alignment of G-tetrad planes. A slight average inclination was found for G-tetrads. The "planar shift" parameter is a measure of the displacement of the center of one tetrad plane with respect to the other. No structurally significant variation in "inclination” or "planar shift” is found. 


\subsubsection{Electronic coupling and hole transfer rates}

As described in section 3.2 Theory, relative rates of HT between two stacked G-tetrads are determined by the ratio of electronic coupling squared (Eq. 3-9). In Table 3-2 we list values of the effective coupling squared derived for each type of G-tetrad stacking geometry by averaging over $1000 \mathrm{MD}$ snapshots examined at the QM level.

The left panel of Figure 3-3, shows time evolution of $V^{2}$ between stacked guanines G2 and G6 in the G-tetrad stack E (Fig. 3-2E). The changes are caused by thermal fluctuations of the structure. As seen, the $V^{2}$ values are extremely sensitive to the stack geometry. Quantum mechanical calculations of the coupling for a single or few structures may provide inaccurate estimates of $V^{2}$ and the related HT rate. Averaging over many thermally accessible conformations is required to obtain reliable values of the effective coupling. This is evident when examining scatter plots of the $V^{2}$ dependence on the twist parameter (Fig. 3-3B). A wide range of $V^{2}$ values are observed for similar values of twist with no strong correlation between $V^{2}$ and the individual parameters that define the mutual position of G-tetrads in the stack. Nevertheless, one can see that the largest coupling values $\left(V^{2}>0.2 \mathrm{eV}^{2}\right)$ of stacked guanines are found for twist values ranging from

Table 3-2. Electronic coupling squared (in $\mathrm{eV}^{2}$ ) between stacked guanine bases in different G-quadruplex structures.

\begin{tabular}{|c|c|c|c|c|c|}
\hline $\begin{array}{c}\text { Stacking } \\
\text { Orientation }^{\mathrm{a}}\end{array}$ & $\begin{array}{c}\text { Partial 5/6-ring } \\
\text { (A) }\end{array}$ & $\begin{array}{l}\text { 5-ring } \\
\text { (B) }\end{array}$ & $\begin{array}{l}\text { Partial 6-ring } \\
\text { (C) }\end{array}$ & $\begin{array}{l}\text { 6-ring } \\
\text { (D) }\end{array}$ & $\begin{array}{l}\text { 5/6-ring } \\
\text { (E) }\end{array}$ \\
\hline$V_{15}^{2}$ & $0.78 \cdot 10^{-3}$ & $2.66 \cdot 10^{-3}$ & $2.79 \cdot 10^{-3}$ & $7.39 \cdot 10^{-3}$ & $71.1 \cdot 10^{-3}$ \\
\hline$V_{26}^{2}$ & $1.38 \cdot 10^{-3}$ & $4.75 \cdot 10^{-3}$ & $3.20 \cdot 10^{-3}$ & $6.27 \cdot 10^{-3}$ & $97.6 \cdot 10^{-3}$ \\
\hline$V_{37}^{2}$ & $1.10 \cdot 10^{-3}$ & $2.93 \cdot 10^{-3}$ & $2.44 \cdot 10^{-3}$ & $5.50 \cdot 10^{-3}$ & $93.4 \cdot 10^{-3}$ \\
\hline$V_{48}^{2}$ & $1.54 \cdot 10^{-3}$ & $2.24 \cdot 10^{-3}$ & $1.97 \cdot 10^{-3}$ & $10.15 \cdot 10^{-3}$ & $74.9 \cdot 10^{-3}$ \\
\hline Mean & $1.18 \cdot 10^{-3}$ & $3.15 \cdot 10^{-3}$ & $2.60 \cdot 10^{-3}$ & $7.33 \cdot 10^{-3}$ & $84.3 \cdot 10^{-3}$ \\
\hline
\end{tabular}



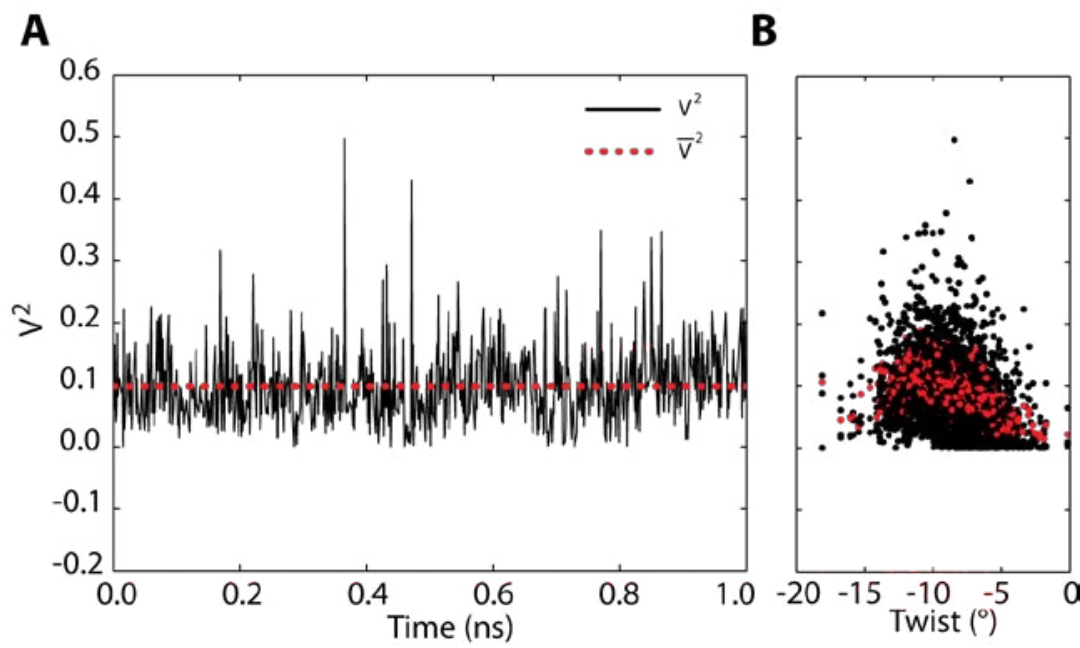

Figure 3-3. (A) Time evolution of electronic coupling squared $V^{2}$. (B) Scatter plots of the $V^{2}$ vs. the twist parameter. In both cases the coupling of G2 and G6 (see Fig. 3-1C) in the $\pi$ stack E is considered.

-14 to -6 degrees.

The non-adiabatic electron transfer rates calculated using Eq. (3-2) are listed in Table 3-3. Rates are found to vary from $26 \mathrm{~ns}^{-1}$ for the Partial 5/6-ring geometry (Fig 3-2A) to 1.9 $\mathrm{ps}^{-1}$ for the 5/6-ring geometry (Fig. 3-2E). The absolute values of $k_{\mathrm{HT}}$ depend strongly on the value of reorganization energy. For example, $k_{\mathrm{HT}}$ decreases by a factor $\sim 2.7$ when $\lambda$ increases by $0.1 \mathrm{eV}$. However, relative HT rates $k_{\text {rel }}$ remain unchanged. A 70 -fold difference is observed in the value of $k_{\text {rel }}$ between the Partial 5/6-ring geometry and the 5/6-ring geometry which is independent of the reorganization energy parameter $\lambda$. Thus, we observe that the 5/6-ring orientation of stacked G-tetrads (Fig 3-2E) facilitates HT significantly as compared with other G-tetrad stacks found experimentally (Fig 3-4). Am-

Table 3-3. Absolute and relative rates of HT between adjacent tetrads in the G-quadruplexes

\begin{tabular}{lrr}
\hline Stacking Orientation $^{\mathrm{a}}$ & $k_{H T}{ }^{\mathrm{b}}\left(\mathrm{ns}^{-1}\right)$ & \multicolumn{1}{c}{$k_{\text {rel }}$} \\
\hline Partial 5/6-ring (A) & 26.0 & 1 \\
5-ring (B) & 69.4 & 2.7 \\
Partial 6-ring (C) & 55.3 & 2.1 \\
6-ring (D) & 163.6 & 6.3 \\
5/6-ring (E) & 1912.2 & 73.5 \\
\hline [a] Codes for stacking orientation refer to the geometries of \\
Figure 3-2. \\
[b] The rates are computed at $\lambda=0.7 \mathrm{eV}$ and $\mathrm{T}=298.15 \mathrm{~K}$
\end{tabular}




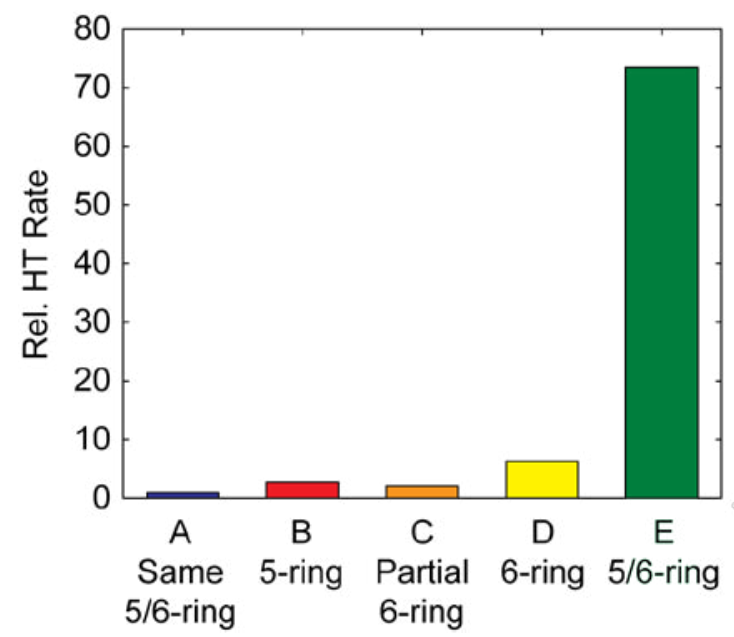

Figure 3-4. Relative HT rates computed for different stacking orientations.

ong geometries that occur within the core of a G-quadruplex, we note that the G-tetrads stacked in a Same-Polarity manner (Fig 3-2A) are found to be less conductive than those stacked in an Opposite-Polarity orientation (Fig B-D).

This 5/6-ring orientation (Fig 3-2E) is a unique geometry that occurs at the interface of some stacked parallel G-quadruplex units. Depending on which ends of the G-quadruplex participate in stacking, dimerization can also result in the formation of a 6-ring geometry (Fig 3-2D) at the stacking interface. By contrast, the Partial 5/6-ring geometry found in the core (Fig 3-2A) of these parallel G-quadruplex units show considerably smaller values of $k_{H T}$. G-tetrad stacking geometries in G-wires formed by long guanine-rich strands of DNA are constrained by the backbone to adopt geometries $A-C$ what are not ideal for CT. Alternatively, it may also be possible to engineer G-tetrads that demonstrate continuous 5/6-ring stacking from the assembly of single guanosine analogs or through modification of the backbone in G-rich DNA.

HT in G-quadruplexes may also occur within a single G-tetrad between hydrogen-bonded guanines. The calculated values of $\overline{V^{2}}$ for these types of HT are listed in Table 3-4. Co- 
Chapter 3: Charge Transport

Table 3-4. Electronic coupling squared between guanine bases in one G-tetrad

\begin{tabular}{ccccccc}
\hline $\mathrm{G} \mathrm{pair}^{\mathrm{a}}$ & $1-2$ & $2-3$ & $3-4$ & $4-1$ & $1-3$ & $2-4$ \\
\hline$V^{2}, \mathrm{eV}^{2}$ & $7.42 \cdot 10^{-6}$ & $5.80 \cdot 10^{-6}$ & $5.05 \cdot 10^{-6}$ & $4.70 \cdot 10^{-6}$ & $5.02 \cdot 10^{-8}$ & $1.46 \cdot 10^{-8}$ \\
\hline
\end{tabular}

mparison of these quantities with data collected in Table 3-3 suggests that the probability of the HT within a G-tetrad are roughly 3 orders of magnitude lower than for HT between stacked nucleobases. 


\subsection{Conclusions}

Using a combined quantum mechanics and molecular dynamics approach we have studied hole transport properties in several experimentally observed G-tetrads geometries with different types of $\pi$-stacking. The effective electronic coupling values between $\pi$ stacked and Hoogsteen bonded guanine nucleobases were derived with the Fragment Charge Difference method applied to adiabatic states obtained using the semi-empirical INDO/S scheme. Our study demonstrates the important role of G-tetrad orientation in electron hole transport within the G-tetrad stacks. The fastest hole hopping between Gtetrad stacks has been for the 5/6-ring geometry commonly found at the interface of stacked G-quadruplex units. The HT rate is at least an order of magnitude slower for other types of G-tetrad structures. Thus, we have identified the stacking configurations of neighboring G-tetrads with enhanced electric conductivity. $V^{2}$ values within a single Gtetrad are at least 2 orders of magnitude smaller than between stacked guanines and therefore hole hopping within individual tetrads should not affect the electron hole mobility in G-quadruplexes. The results of this study provide guidance for the design of conductive G-wire systems. 


\title{
Chapter 4:
}

\section{Engineering the Sugar Backbone: Locked Nucleic Acid}

\begin{abstract}
This chapter was largely adapted from the authors work: Li, Z.*, Lech, C.J.*, Phan, A.T. (2014) Sugar-modified G-quadruplexes: fffects of LNA-, 2'F-RNA- and 2'F-ANA-guanosine chemistries on G-quadruplex structure and stability. Nucleic Acids Research, [in press] (* Denotes co-first authorship) and unpublished work including contributions from Brahim Heddi and Michael Adrian.

Author Contributions: DNA synthesis, purification and thermal denaturing experiments were carried out by L.Z.; Data analysis of thermal denaturing experiments was performed by C.J.L.; 1D NMR experiments were performed by C.J.L. and L.Z.; 2D NMR Experiments were performed by B.H, M.A. and L.Z.; NMR assignments were performed by M.A. and L.Z.; The NMR solution structure of the L4 sequence was computed by M.A. and L.Z. and B.H.; The above mentioned manuscript was written by C.J.L, L.Z. and A.T.P; All authors contributed to the conceptualization and planning of experiments.
\end{abstract}

\subsection{Introduction:}

The introduction of alternative chemistries into G-quadruplexes can be advantageous, making them more suitable for pharmaceutical or engineering applications. Modification to the base $(82,107,111)$ or sugar-phosphate backbone $(106,107,130,222-224)$ can have beneficial effects on the stability, kinetics, enzymatic digestion resistance, and cellular uptake of biologically active G-quadruplexes. One alternative nucleic acid chemistry that has received notable attention is Locked Nucleic Acid (LNA) (225), a ribonucleotide analogue with a 2'-O,4'-C-methylene linkage (Fig. 4-1A). Introduction of LNA can improve oligonucleotide stability towards enzyme digestion as well as the thermal stability of duplexes and triplexes $(225,226)$. Previous studies have shown that LNAmodifications can greatly enhance the RNA cleaving rate of a DNAzyme (227). Additionally, LNA is soluble in water and non-toxic $(104,124)$. 


\section{A}

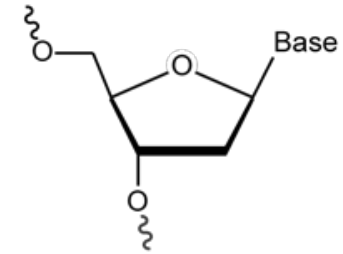

DNA

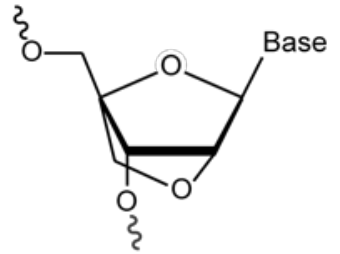

LNA
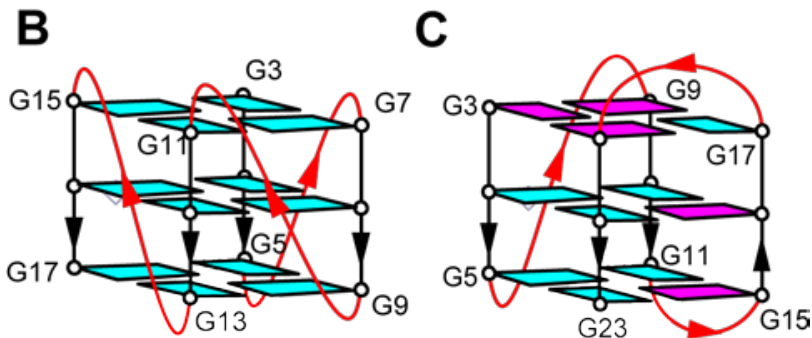

Figure 4-1. Introduction to LNA and G-quadruplexes: (A) Sugar chemistries of DNA and LNA. G-quadruplex scaffolds of the (B) propeller-type (4+0) G-quadruplex formed by the sequence $d\left[T_{2}\left(G_{3} T\right)_{4}\right]$ and the (C) hybrid $(3+1)$ G-quadruplex formed by the sequence $d\left[T_{2}\left(G_{3} T_{2} A\right)_{3} G_{3} A\right]$. Guanines are colored based on their syn (magenta) or anti (cyan) conformation.

In the context of G-quadruplexes, it has been reported that the introduction of LNA modified guanosine $\left({ }^{\mathrm{LNA}} \mathrm{G}\right)$ stabilizes tetramer G-quadruplexes formed by the sequences $d\left[T^{\mathrm{LNA}} \mathrm{G}_{3} \mathrm{~T}\right], \mathrm{d}\left[\mathrm{T}\left(\mathrm{G}^{\mathrm{LNA}} \mathrm{G}\right)_{2} \mathrm{~T}\right]$ and $d\left[\mathrm{~T}^{\mathrm{LNA}} \mathrm{G}_{4} \mathrm{~T}\right](126,127) .{ }^{\mathrm{LNA}} \mathrm{G}$ is previously observed to favor an anti glycosidic conformation of the base (125) and previous studies have taken advantage of this preference to engineer G-quadruplex folding topology $(65,228)$. Substitutions of ${ }^{\mathrm{LNA}} \mathrm{G}$ into positions that adopt a syn conformation tend to push structural equilibrium towards a parallel conformation where all guanines adopt an anti conformation $(125,228,229)$. Incorporation of ${ }^{\mathrm{LNA}} \mathrm{G}$ has also been used to enhance the inhibitory properties of biologically active G-quadruplex molecules that demonstrate activity against HIV-1 (230) or thrombin (231).

Considering the demonstrated potential of LNA for enhancing the drug-like properties of G-quadruplexes, we set out on a systematic study to characterize the effects of LNA 
incorporation into G-quadruplex DNA. In this study, two G-quadruplex-forming sequences were site-specifically modified at each guanine residue with ${ }^{\text {LNA }}$. Singleposition substitutions were made into: 1) The intramolecular parallel-stranded “(4+0)” Gquadruplex formed by the sequence $\mathrm{d}\left[\mathrm{T}_{2}\left(\mathrm{G}_{3} \mathrm{~T}\right)_{4}\right]$ (182), termed "PS-series" (Fig. 4-1B). This (4+0) G-quadruplex structure contains three single-nucleotide (1-nt) propeller loops with all guanine bases adopting an anti glycosidic conformation. 2) The intramolecular “(3+1)" hybrid G-quadruplex formed by the sequence $d\left[T_{2}\left(G_{3} T_{2} A\right)_{3} G_{3} A\right]$ (28), termed “HT-series” (Fig. 4-1C). The (3+1) G-quadruplex of the HT-series contains 3-nt edgewise and propeller loops with guanine bases in both syn and anti conformations. These two sequences were chosen to explore a range of G-quadruplex structural environments.

The folding topology and thermal stability of G-quadruplexes formed by ${ }^{\text {LNA }}$ G-modified sequences (Table 4-1) is determined through a series of nuclear magnetic resonance (NMR) spectroscopic, circular dichroism (CD) spectroscopic, and thermal denaturing studies (Appendix II - Protocols - Chapter 4). The high-resolution NMR solution structure of a LNA-modified (3+1) G-quadruplex forming sequence is determined, providing structural insight into observations from our biophysical studies. This systematic study reveals important and novel limitations of LNA incorporation into Gquadruplex nucleic acid. 
Table 4-1. DNA Sequences and Thermal Stability

\begin{tabular}{|c|c|c|c|}
\hline \multirow{2}{*}{ Name $^{\mathrm{a}}$} & \multirow{2}{*}{ Sequence $\left(5^{\prime} \rightarrow 3^{\prime}\right)^{\mathrm{b}}$} & \multicolumn{2}{|c|}{$\left({ }^{\circ} \mathrm{C}\right)^{\mathrm{C}}$} \\
\hline & & $\mathrm{T}_{\mathrm{m}}$ & $\Delta \mathrm{T}_{\mathrm{m}}$ \\
\hline$(4+0)$ native & TTGGGTGGGTGGGTGGGT & $79.0 \pm 0.5$ & - \\
\hline PS-L3 & TTLGGTGGGTGGGTGGGT & $84.0 \pm 0.4$ & 5.0 \\
\hline PS-L4 & TTGLGTGGGTGGGTGGGT & $87.4 \pm 0.3$ & 8.4 \\
\hline PS-L5 & TTGGLTGGGTGGGTGGGT & $36.5 \pm 0.4$ & -42.5 \\
\hline PS-L7 & TTGGGTLGGTGGGTGGGT & $86.4 \pm 0.1$ & 7.4 \\
\hline PS-L8 & TTGGGTGLGTGGGTGGGT & $82.2 \pm 0.2$ & 3.2 \\
\hline PS-L9 & TTGGGTGGLTGGGTGGGT & $32.5 \pm 0.1$ & -46.5 \\
\hline PS-L11 & TTGGGTGGGTLGGTGGGT & $87.0 \pm 0.1$ & 8.0 \\
\hline PS-L12 & TTGGGTGGGTGLGTGGGT & $81.8 \pm 0.1$ & 2.8 \\
\hline PS-L13 & TTGGGTGGGTGGLTGGGT & $32.0 \pm 0.5$ & -47.0 \\
\hline PS-L15 & TTGGGTGGGTGGGTLGGT & $85.3 \pm 0.1$ & 6.3 \\
\hline PS-L16 & TTGGGTGGGTGGGTGLGT & $83.0 \pm 0.1$ & 4.0 \\
\hline PS-L17 & TTGGGTGGGTGGGTGGLT & $82.4 \pm 0.1$ & 3.4 \\
\hline$(3+1)$ native & TTGGGTTAGGGTTAGGGTTAGGGA & $56.3 \pm 0.2$ & - \\
\hline HT-L3 & TTLGGTTAGGGTTAGGGTTAGGGA & $54.3 \pm 0.3$ & -2.0 \\
\hline HT-L4 & TTGLGTTAGGGTTAGGGTTAGGGA & $60.5 \pm 0.0$ & 4.2 \\
\hline HT-L5 & TTGGLTTAGGGTTAGGGTTAGGGA & $55.8 \pm 0.4$ & -0.6 \\
\hline HT-L9 & TTGGGTTALGGTTAGGGTTAGGGA & - & - \\
\hline HT-L10 & TTGGGTTAGLGTTAGGGTTAGGGA & $58.2 \pm 0.4$ & 1.9 \\
\hline HT-L11 & TTGGGTTAGGLTTAGGGTTAGGGA & $57.2 \pm 0.3$ & 0.9 \\
\hline HT-L15 & TTGGGTTAGGGTTALGGTTAGGGA & - & - \\
\hline HT-L16 & TTGGGTTAGGGTTAGLGTTAGGGA & - & - \\
\hline HT-L17 & TTGGGTTAGGGTTAGGLTTAGGGA & $54.0 \pm 0.4$ & -2.3 \\
\hline HT-L21 & TTGGGTTAGGGTTAGGGTTALGGA & - & - \\
\hline HT-L22 & TTGGGTTAGGGTTAGGGTTAGLGA & - & - \\
\hline HT-L23 ${ }^{\mathrm{d}}$ & TTGGGTTAGGGTTAGGGTTAGGLA & $59.4 \pm 0.1$ & 3.0 \\
\hline
\end{tabular}

[a] "HT-“ series denotes a modified (3+1) G-quadruplex forming sequence while "PS-" series denotes a modified (4+0) G-quadruplex forming sequence.

[b] Residues with LNA-modified guanosine are denoted as (L)

[c] Thermal stability data was obtained via UV melting (for HT-series) and CD melting (for PS-series) experiments. Salt conditions were (20 mM KPi) for the HT-series and (1.1 mM KPi) for the PS-series. Thermal stability data for the HTseries is presented for sequences which demonstrate a single species in NMR. The " \pm ” values indicate the hysteresis between heating and cooling curves.

[d] Sequence contains a small secondary melting transition at low temperature range 


\subsection{Results and Discussion:}

4.2.1: Substitution of ${ }^{\mathrm{LNA} G}$ into a $(4+0)$ G-quadruplex: LNA modifications are detrimental when substituted prior to short propeller loops.

G-quadruplex formation of ${ }^{\text {LNA }} \mathrm{G}$-modified sequences was evaluated by monitoring the imino proton region (10.5 - 12.5 p.p.m.) of ${ }^{1} \mathrm{H}$ NMR spectra (232). Chemical shift patterns in this region can be used to determine the number of G-quadruplex conformations present. Similarity between NMR spectra is a strong indicator that sequences adopt a similar folding topology. Such consideration is important as small changes in sequence can have large effects on the conformations adopted by Gquadruplex forming sequences $(23,233)$. CD spectroscopy was also used to probe Gquadruplex folding topology based on well-characterized patterns in CD spectra (234). Additionally, thermal stability of ${ }^{\mathrm{LNA}} \mathrm{G}$-modified sequences was evaluated through a series of UV and CD melting experiments. The melting temperature $\left(T_{m}\right)$ of these sequences is presented as a useful quantitative metric for comparing the thermal stability of modified sequences.

Within the (4+0) G-quadruplex-forming PS-series, nine of the twelve single-position ${ }^{\text {LNA }} \mathrm{G}$-modified sequences were observed to form a single G-quadruplex species in imino proton NMR spectra and demonstrated spectra which were similar to the native sequence (Fig 4-2.A). CD spectra of these sequences were characteristic of a (4+0) G-quadruplex conformation, with a maximum around $260 \mathrm{~nm}$ and a minimum around $240 \mathrm{~nm}$ (Fig. 42.B). Conversely, substitution of ${ }^{\mathrm{LNA}} \mathrm{G}$ into positions G5, G9, and G13 lead to multiple conformations in NMR spectra and a reduction in the intensity of CD spectra at $260 \mathrm{~nm}$ (Fig. 4-2). Sequences that formed multiple conformations also demonstrated extreme inst- 
A

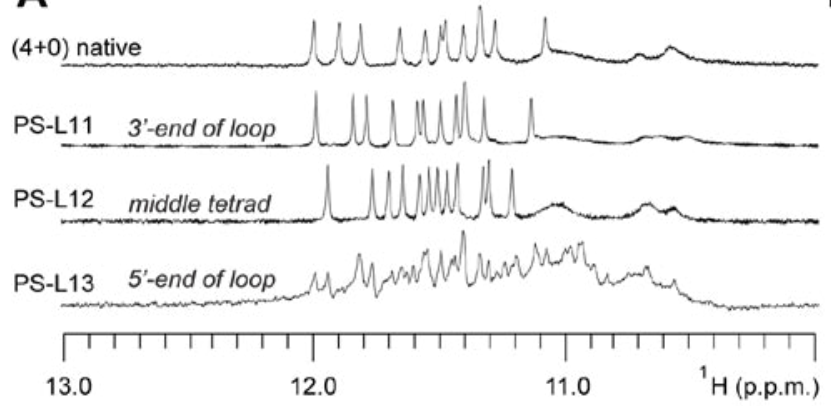

B

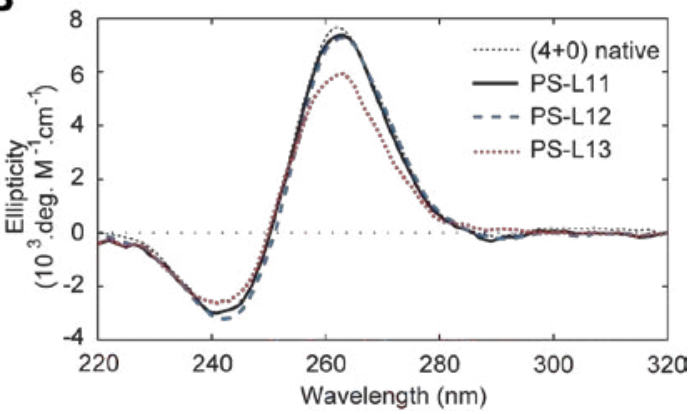

Figure 4-2. LNA modifications disrupt the (4+0) G-quadruplex conformation of PS-series sequences in a structurally dependent manner: (A) NMR imino proton and (B) CD spectra of select PS-series sequences containing single-position LNA substitutions on the 3'-end of loop, middle tetrad, or 5'-end of loop.

ability with a change in melting temperature $\left(\Delta \mathrm{T}_{\mathrm{m}}\right)$ exceeding $-40^{\circ} \mathrm{C}$ (Fig $4-3$ ). Alternatively, sequences that formed a single conformation displayed $\Delta \mathrm{T}_{\mathrm{m}}$ values of $+2.8^{\circ} \mathrm{C}$ and $+8.4^{\circ} \mathrm{C}$ in $\mathrm{CD}$ melting studies

We note the unfavorable substitutions occur at residues G5, G9 and G13 which are located upstream, at the 5'-end, of 1-nt propeller loops within this intramolecular $(4+0)$ scaffold. These results suggest the incompatibility of LNA incorporation into residues preceding short propeller loops. Considering this observation, we proceeded to explore the role of loop length in the ${ }^{\mathrm{LNA}} \mathrm{G}$-induced destabilization from modifications upstream

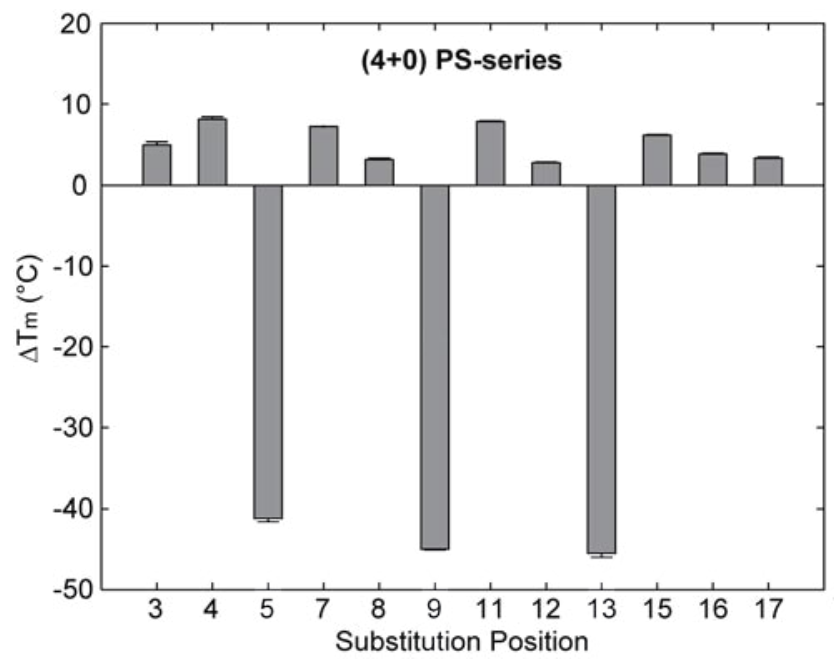

Figure 4-3. $\Delta \mathrm{T}_{\mathrm{m}}$ of PS-series sequences containing single-position ${ }^{\mathrm{LNA}} \mathrm{G}$ modifications determined by $\mathrm{CD}$ melting experiments. 
of propeller loops in the (4+0) scaffold. We expanded the 1-nt Thymine (T) loop at position T10 to TT and TTT in both the native and ${ }^{\mathrm{LNA}}$ G9-modified PS-L9 sequences. The effects of loop elongation were monitored using NMR and CD melting experiments (Fig. 4-4). The $T_{m}$ values of the loop-extended native sequence and PS-L9 sequences converge as the loop length increases. At a loop length of TTT, the difference in $T_{m}$ between the two sequences converged to a mere $5^{\circ} \mathrm{C}$ compared to a $46^{\circ} \mathrm{C}$ difference in sequences containing a 1-nt loop. These changes in $T_{m}$ values coincide with the emergence of a single conformation in the NMR spectrum of the ${ }^{\text {LNA }}$ G9-modified TTTloop sequence and a single folding topology maintained for the unmodified TTT-loop sequence.

In this study, we show that ${ }^{\mathrm{LNA}} \mathrm{G}$ substitution upstream of short propeller loops is destabilizing to G-quadruplex structure. ${ }^{\text {LNA }}$ G substitution to positions G5, G9 and G13 in the (4+0) G-quadruplex is observed to be disruptive despite the anti glycosidic conforma-

A

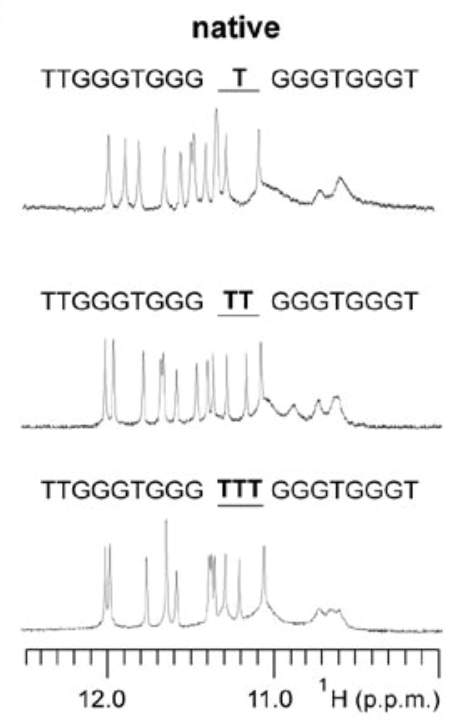

PS-L9
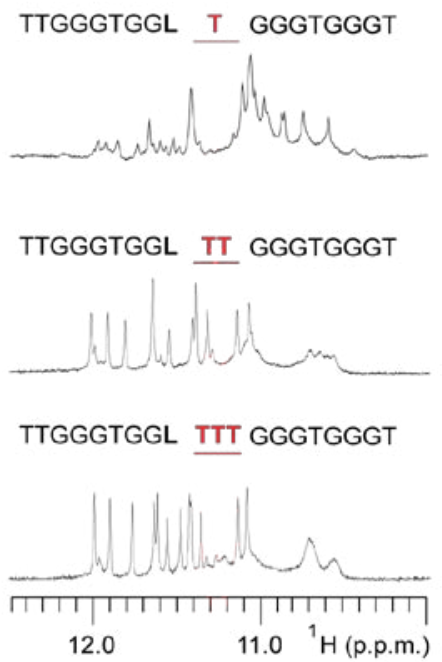

B

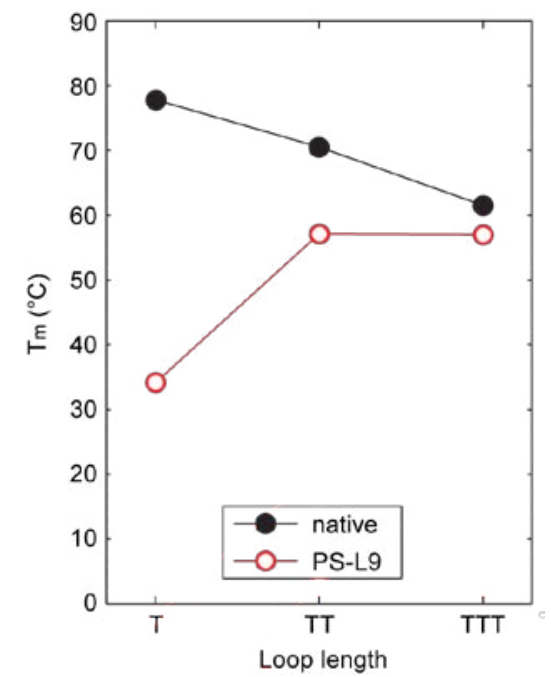

Figure 4-4. Effect of loop length on thermal stability: (A) NMR Imino proton spectra. The 1-nt T10 loop of the native (4+0) G-quadruplex forming sequence and PS-L9 containing a LNA substitution at position 9 were elongated. Sequences containing a loop of T, TT, and TTT are shown. (B) $\mathrm{T}_{\mathrm{m}}$ of the loop-elongated native (black) and PS-L9 (red) sequences. 
-tion of the substituted guanine base. Elongation of these 1-nt propeller loops is shown to reduce the detrimental effects of ${ }^{\mathrm{LNA}} \mathrm{G}$ substitutions. It is likely that the conformational constraints on the sugar-backbone geometry imposed by the short propeller loop are incompatible with the conformationally restricted LNA sugar-backbone. The position dependent nature of ${ }^{\text {LNA }} \mathrm{G}$ substitutions into anti-position guanines is in direct contrast to more versatile nucleoside such as 2'-F-guanoisine and 2'-F-ANA-guanosine (see Chapter 5). The disruptive effect of ${ }^{\text {LNA }}$ G-modification at the 5'-end of short loops constitutes an important consideration for future design of G-quadruplexes containing LNA modifications.

\subsubsection{Substitution of ${ }^{\mathrm{LNA}} \mathrm{G}$ into a $(3+1)$ G-quadruplex: LNA modifications generally affect structure in a syn/anti dependent manner}

Similar to our study of the (4+0) G-quadruplex scaffold, we systematically substituted

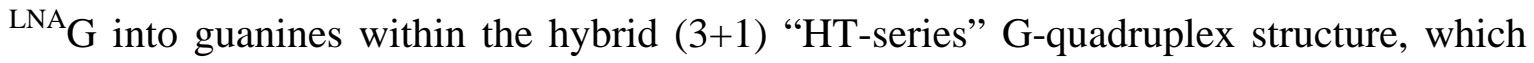
contains guanine bases in both syn and anti conformations (Fig 4-1C). Within the HTseries, seven of the twelve modified sequences demonstrated a single conformation as determined by NMR spectra (Fig. 4-5A). CD spectra of these sequences demonstrated a $(3+1)$ profile similar to the native sequence (Fig. 4-5B). Conversely, the other five ${ }^{\mathrm{LNA} G}$ substitutions induced multiple conformations as observed in NMR spectra (Fig 4-5A). The CD spectra of these five modified sequences exhibit a profile that deviates from the (3+1) native sequence, with an increase in absorbance at $260 \mathrm{~nm}$ and a decrease at 295 nm (Fig. 4-5C).

Within the $(3+1)$ G-quadruplex, six of the seven sequences displaying a single G-quadru- 

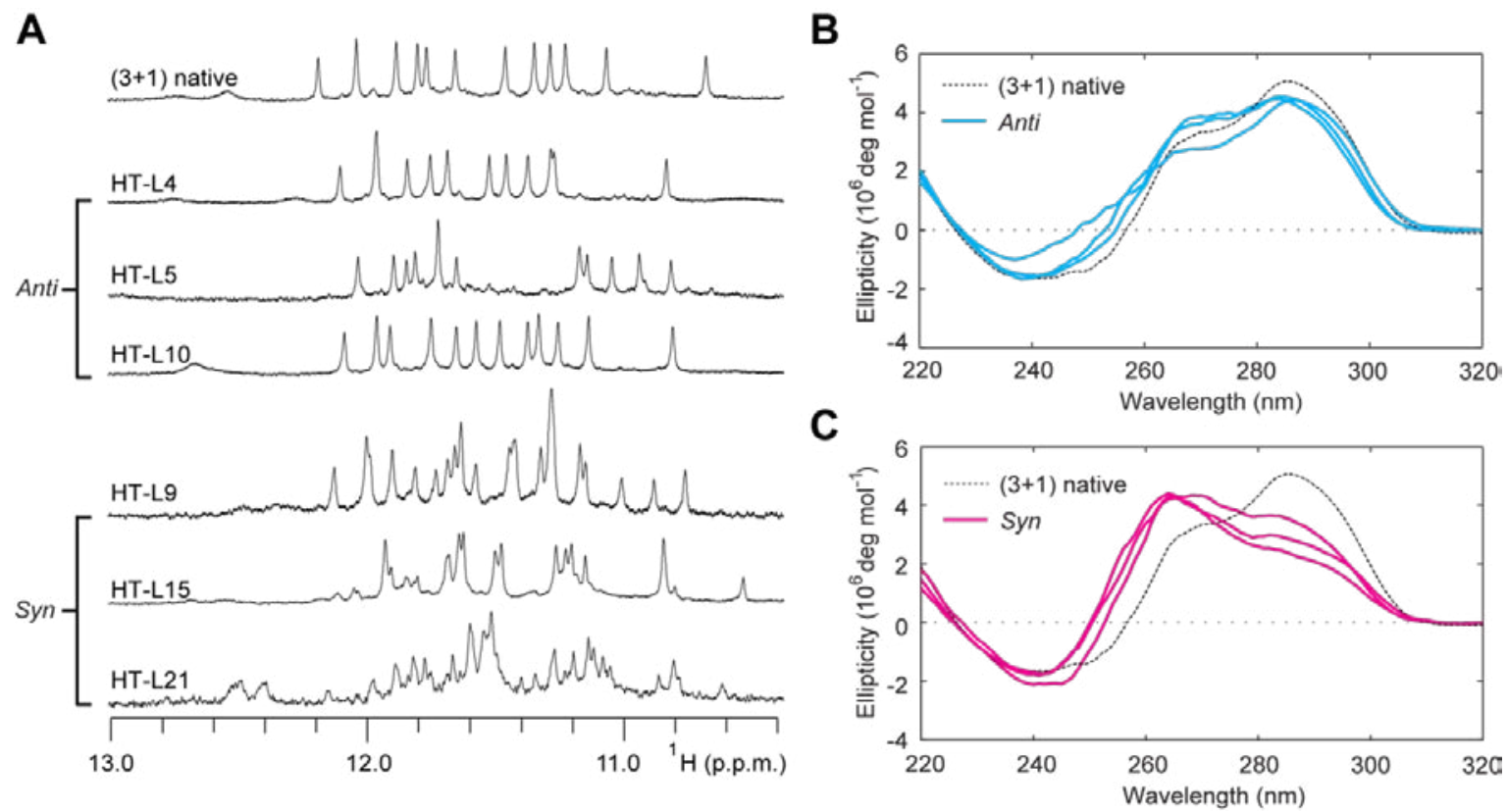

Figure 4-5. The syn/anti preference of LNA substitutions into the HT-series: (A) NMR imino proton spectra and (B-C) CD spectra are shown for select sequences containing single position LNA substitutions to (B) anti positions and (C) syn positions.

plex conformation contained ${ }^{\mathrm{LNA}} \mathrm{G}$ substitutions to guanosine in an anti glycosidic conformation. Substitution with ${ }^{\mathrm{LNA}} \mathrm{G}$ produced a mixture of effects on the thermal stability of modified sequences (Fig. 4-6). Substitution into anti guanines displayed $\Delta \mathrm{T}_{\mathrm{m}}$ values in the range of $-2.3^{\circ} \mathrm{C}$ to $+4.2^{\circ} \mathrm{C}$. Four of the five ${ }^{\mathrm{LNA}} \mathrm{G}$ substitutions that induced multiple conformations with made to syn position guanines. Substitution to syn-position G3 led to a destabilization, with a $\Delta \mathrm{T}_{\mathrm{m}}$ of $-1.4^{\circ} \mathrm{C}$ despite the observation of a single $(3+1)$ conformation. Comparison of $\mathrm{T}_{\mathrm{m}}$ values for sequences that demonstrate multiple species is complicated by complex melting curves and an uncertainty about which structural transition is being analyzed. For this reason we don’t discuss these sequences in detail.

It is generally observed that substitution of ${ }^{\mathrm{LNA}} \mathrm{G}$ into syn-position guanines disrupts the folding topology of the G-quadruplex and lead to co-existence of multiple species while anti positions substitutions are generally tolerated. This is not surprising as ${ }^{\mathrm{LNA}} \mathrm{G}$ has been reported to favor an anti conformation (65). Previous studies have observed that ${ }^{\text {LNA }} \mathrm{G}$ su- 


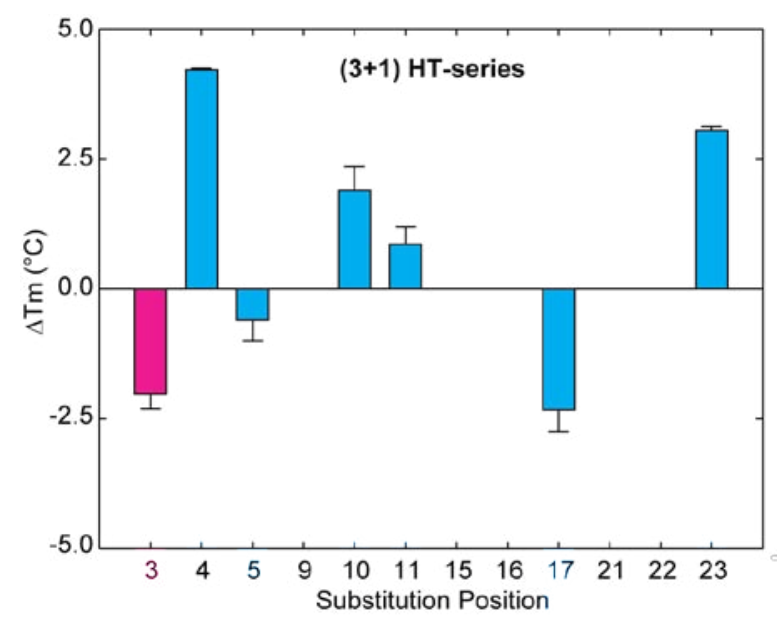

Figure 4-6. $\Delta \mathrm{T}_{\mathrm{m}}$ upon LNA modification to HT-series G-quadruplexes: Values are plotted for sequences containing single-position LNA substitutions that demonstrated a single conformation. Cyan and magenta indicate the anti or syn conformation respectively of the substituted guanine in the unmodified sequence.

bstitution into anti guanines stabilize G-quadruplexes $(126,127)$ while substitution to syn positions has a destructive effect on the native G-quadruplexes conformation $(65,125,229)$.

Despite the general syn/anti dependence the ${ }^{\mathrm{LNA}} \mathrm{G}$ modifications, we note some important exceptions to this trend. Within the (4+0) G-quadruplex-forming PS-series discussed above, substitutions were generally stabilizing, with increases in $T_{m}$ observed between $+2.8^{\circ} \mathrm{C}$ and $+8.4^{\circ} \mathrm{C}$. However, ${ }^{\mathrm{LNA}} \mathrm{G}$ modifications directly upstream of 1 -nt propeller

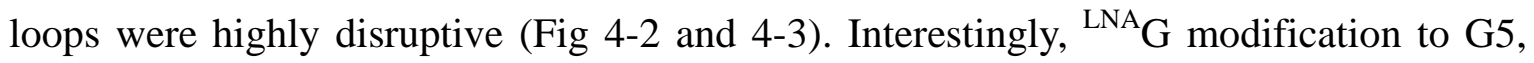
G11, and G17 within the HT-series, located upstream of a 3-nt propeller or edge-wise loop, were the least favorable anti-position substitutions tested in the (3+1) G-quadruplex with $\Delta \mathrm{T}_{\mathrm{m}}$ values of $-2.1^{\circ} \mathrm{C},+0.4^{\circ} \mathrm{C}$ and $-3.2^{\circ} \mathrm{C}$ respectively (Fig 4-6). These results, in conjunction with results of our loop-elongation experiments (Fig 4-4), suggest that the negative effects of ${ }^{\mathrm{LNA}} \mathrm{G}$ modification upstream of loops may not be limited to short propeller loops. 

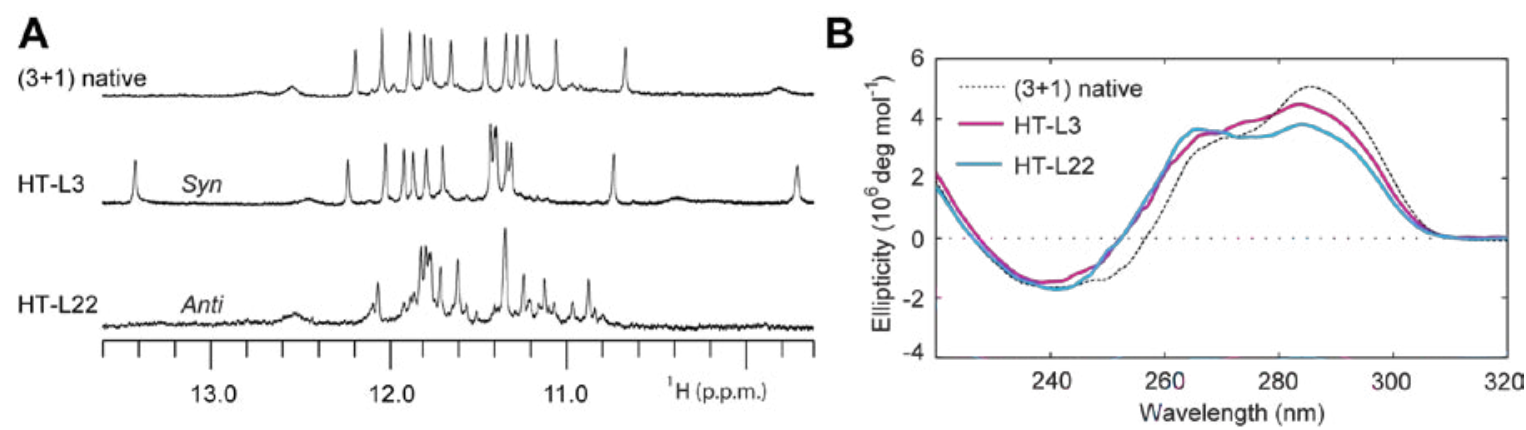

Figure 4-7. The (A) NMR imino proton spectra and (B) CD spectra are presented for the syn-position modified HT-L3 sequence and the anti-position modified HT-L22 sequence

Other exceptions to the syn/anti dependence of ${ }^{\mathrm{LNA}} \mathrm{G}$ modifications are also observed.

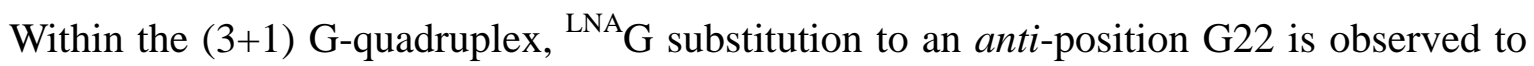
disrupt the native conformation (Fig 4-7). It is not clear why G22 does not tolerate ${ }^{\mathrm{LNA}} \mathrm{G}$ modification, as modification to other central-tetrad anti-position guanines G4 and G10 does not disturb the $(3+1)$ conformation. Additionally, a single instance of syn-position substitution to residue G3 unexpectedly resulted in an undisrupted $(3+1)$ conformation (Fig 4-7). The NMR spectra of this sequence contains sharp peaks at $9.7 \mathrm{ppm}$ and 13.4 ppm not visible in the $(3+1)$ native sequence suggesting enhanced external base pairing (Fig 4-7A). However, this syn-substitution comes at a cost of reduced thermal stability (Fig. 4-6). The exceptions to the generalized glycosidic-dependence of ${ }^{\mathrm{LNA}} \mathrm{G}$ substitutions observed in this work are important considerations when incorporating these modifications into G-quadruplex nucleic acids. 


\subsubsection{LNA modification induces local backbone rearrangement: NMR solution structure of a $(3+1)$ G-quadruplex containing a single ${ }^{\mathrm{LNA}} \mathrm{G}$ modification}

The experiments and results in this section are largely the work of collaborators Li, Z., Adrian, M., and Heddi, B. A summary of these results in relation to the over-all goal of our LNA studies is presented.

The NMR solution structure of one of the $(3+1)$ G-quadruplex-forming HT-series sequences was solved in order to investigate the effects of a single ${ }^{\mathrm{LNA}} \mathrm{G}$ modification on G-quadruplex structure in atomic detail. We choose to study the sequence containing an

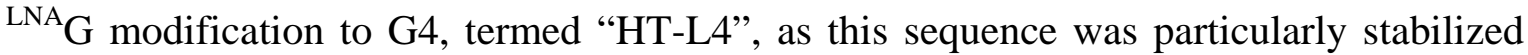
upon ${ }^{\mathrm{LNA}} \mathrm{G}$-modification and demonstrated a well resolved ${ }^{1} \mathrm{H}$ NMR spectra.

We performed unambiguous assignments of aromatic H8 protons for seven of the guanines in HT-L4 by site-specific deuterium labeling. The remaining H8 and H1 protons were assigned by sequential connectivity of the H8-H1' region in NOESY experiments and through-bond correlation of $\mathrm{H} 1-\mathrm{H} 8$ protons in ${ }^{1} \mathrm{H}_{-}{ }^{13} \mathrm{C}$ HMBC experiments. NOEs between $\mathrm{H} 1$ imino and $\mathrm{H} 8$ protons in $\mathrm{H}_{2} \mathrm{O}$ NOESY experiments are used to determine the alignment of guanines within G-tetrads. These series of NMR experiments indicate that HT-L4 adopts the same (3+1) G-quadruplex conformation as the unmodified sequence (Fig 4-1B). This folding topology is in agreement with a CD spectrum that is characteristic of a $(3+1)$ conformation.

A collection of NMR restraints including NOE-derived distance restraints were used to compute the NMR solution structure of the $(3+1)$ G-quadruplex formed by the HT-L4 sequence (Fig 4-8A). Similar to the native sequence, base pairing is observed between $\mathrm{T} 1 \bullet \mathrm{A} 20$ and T13•A24, with these bases stacking on external tetrads (Fig. 4-8B). The most pronounced difference between the $(3+1)$ native and HT-L4 structures is a rearrangement 
of the sugar and phosphate-backbone geometry at the ${ }^{\text {LNA } G 4-t o-G 5}$ di-nucleotide step (Fig 4-8C and Fig 4-9). The sugar pucker of ${ }^{\mathrm{LNA}} \mathrm{G} 4$ adopts a C3'-endo conformation as expected for a LNA sugar moiety. The $\chi$ and $\delta$ angles of ${ }^{\text {LNA }}$ G4 vary notably from G4 of the native structure. The $\chi$ angle of ${ }^{\mathrm{LNA}} \mathrm{G} 4$ adopts a value of $\sim-159^{\circ}$ compared to $\sim-124^{\circ}$ in the native structure, while a value of $\sim 67^{\circ}$ is observed for $\delta$ compared to $\sim 123^{\circ}$ in the native G-quadruplex. Both the $\chi$ and $\delta$ dihedral angles of G4 are well converged in our structure (Fig. 4-9). Additionally, the relative $\chi$ values for ${ }^{\mathrm{LNA}} \mathrm{G}$ and ${ }^{\mathrm{DNA}} \mathrm{G}$ are in good agreement with values predicted by quantum chemical computations (125). The LNAinduced changes to sugar pucker as well as $\chi$ and $\delta$ dihedral angles cause a projection of the backbone that is notably different then the native structure (Fig 4-8C). Despite this different orientation, the $\alpha, \beta, \gamma$ and $\delta$ dihedral angles in ${ }^{\text {LNA }}$ G4-to-G5 step were largely unaffected, with the $\alpha / \gamma$ dihedrals adopting a similar $\mathrm{g}^{-} / \mathrm{g}^{+}$orientation as observed in the native structure (Fig 4-9).

As sugar pucker is related to backbone conformation, it is not surprising that reorientation

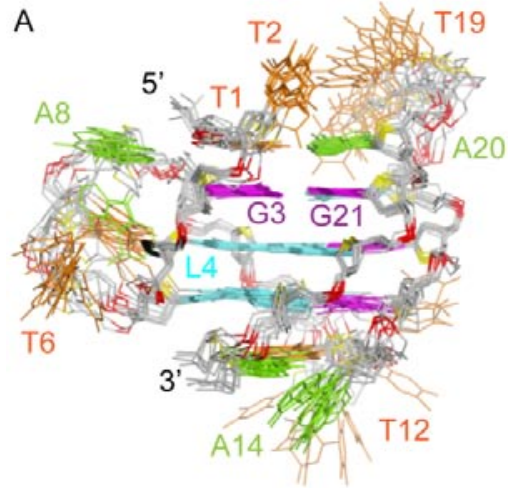

B

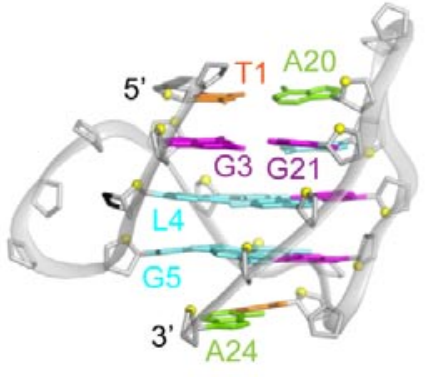

C

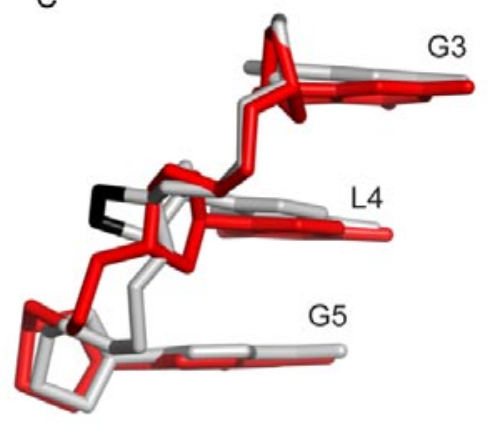

Figure 4-8. NMR Solution structure of ${ }^{\mathrm{LNA}} \mathrm{G} 4$-modified HT-series sequence: (A) Alignment of the 10 lowest energy structures. (B) Representative ribbon structure highlighting the (3+1) conformation and $\mathrm{A} \bullet \mathrm{T}$ base pairing on the 3' and 5'-ends. The sugar bridge of ${ }^{\mathrm{LNA}} \mathrm{G} 4$ is shown in black. Guanine bases are colored by anti (cyan) and syn (magenta) glycosidic conformation. Adenines (green) and thymines (orange) are colored by type. Atoms are colored as such: Backbone and sugar (grey); O4' (yellow); and P (red). (C) Local structural deviation in the sugar-backbone of the ${ }^{\mathrm{LNA}} \mathrm{G} 4$ modified sequence (grey) compared to the unmodified HT-series structure (red). 


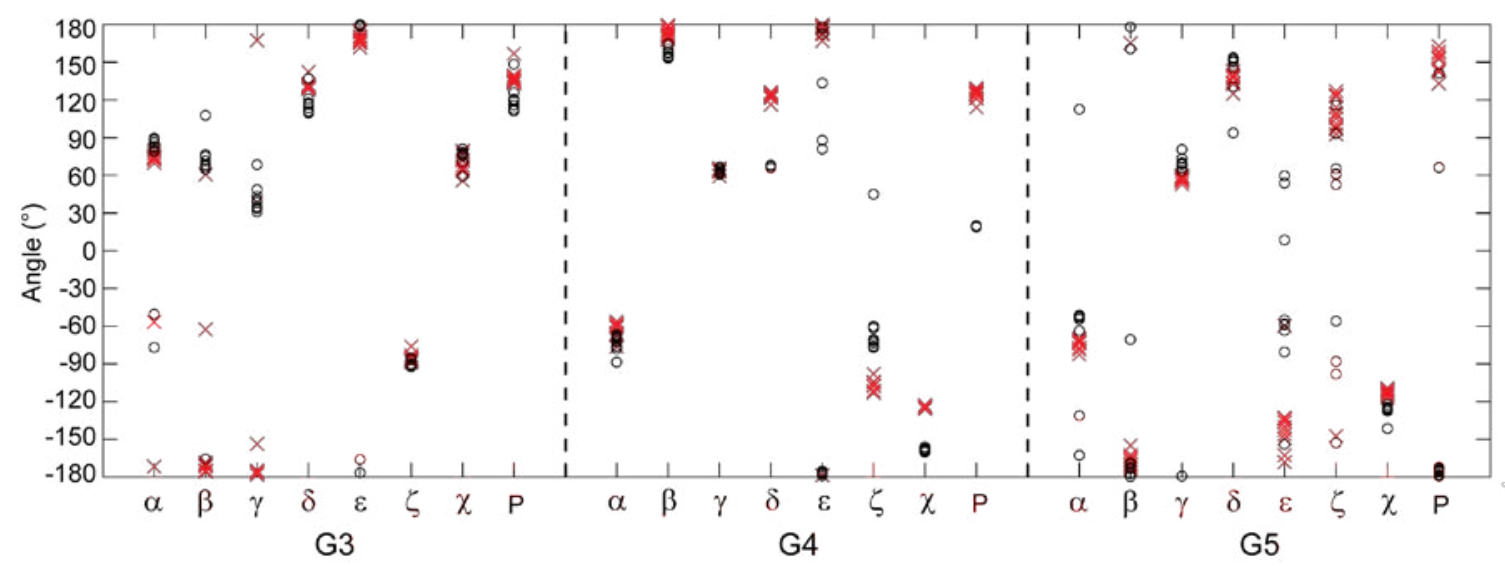

Figure 4-9. Dihedral angle comparison of the (3+1) native structure and the ${ }^{\mathrm{LNA}} \mathrm{G} 4$-modified $(3+1) \mathrm{G}$ quadruplex forming HT-L4 sequence: Plots of standard backbone angles $(\alpha, \beta, \gamma, \delta, \varepsilon, \zeta)$, glycosidic angle $(\chi)$ and Pseudo-rotation angle of the sugar $(\mathrm{P})$ for the $\mathrm{G} 3 \rightarrow \mathrm{G} 5$ residues in the $(3+1)$ native (red $\mathrm{x}$ ) and HT-L4 (black o) sequences.

occurs around the modified residue as previously reported $(126,127)$. Similar values of $\chi$ and $\delta$ dihedral angles have been observed upon ${ }^{\mathrm{LNA}} \mathrm{G}$ modification into a tetramer Gquadruplex (126). Our NMR studies of the HT-L4 sequence reveal that LNA incorporation can induce local re-arrangement of the sugar-phosphate backbone, especially downstream in the linkage to the 3'-neighboring residue. These observations provide a structural explanation for the observed intolerance of ${ }^{\mathrm{LNA}} \mathrm{G}$ modification upstream to short-propeller loops in G-quadruplex DNA as such rearrangement may be incompatible with the fold-back geometry required of a short-propeller loop. 


\subsection{Conclusions:}

Through systematic single-position LNA modifications on well-defined intramolecular $(4+0)$ and $(3+1)$ G-quadruplex scaffolds, we discover that modification of ${ }^{\text {LNA }} \mathrm{G}$ directly upstream of short propeller loops is highly disruptive to G-quadruplex formation. The NMR solution structure of a (3+1) G-quadruplex containing a single LNA modification is solved, revealing local backbone rearrangement downstream of the ${ }^{\mathrm{LNA}} \mathrm{G}$ modification and providing a structural explanation for the severe destabilization observed when ${ }^{\text {LNA } G}$ substitution precedes short-propeller loops in our studies. ${ }^{\text {LNA }} \mathrm{G}$ substitutions are generally tolerated in a manner dependent on the syn or anti glycosidic conformation of the guanine base. Substitution of ${ }^{\mathrm{LNA}} \mathrm{G}$ into most anti positions leads to more stable G-quadruplex, while substitution into most syn positions generally disrupts the native $(3+1)$ Gquadruplex conformation. However, we indentify some noteworthy exceptions to these generalizations in the course of our study. The insight gained from this work will be valuable to the future design of LNA-modified G-quadruplexes for pharmaceutical and engineering applications. 


\title{
Chapter 5:
}

\section{Engineering the Sugar Backbone: 2'-F-Guanosine and 2'-F-ANA-Guanosine Modifications}

\begin{abstract}
This chapter was largely adapted from the authors works: Li, Z.*, Lech, C.J.*, Phan, A.T. (2014) Sugar-modified G-quadruplexes: effects of LNA-, 2'F-RNA and 2'F-ANA-guanosine chemistries on G-quadruplex structure and stability. Nucleic Acids Research, [in press] (* Denotes co-first authorship) and Lech, C.J., ${ }^{*}$ Zhe, L., ${ }^{*}$ Heddi, B., Phan, A.T. (2012) 2'-F-ANA-guanosine and 2'-F-guanosine as powerful tools for structural manipulation of G-quadruplexes. Chemical Communications. 48. 11425-11427. (* Denotes co-first authorship)

Author Contributions: DNA synthesis, purification and thermal denaturing experiments were carried out by L.Z.; Data analysis of thermal denaturing experiments was performed by C.J.L.; 1D NMR experiments were performed by C.J.L. and L.Z.; All authors contributed to the conceptualization and planning of experiments as well as preparation of manuscripts. We would like to thank Mee Chea Chin for her participation in the early stages of this work
\end{abstract}

\subsection{Introduction:}

The incorporation of alternative nucleic acid chemistries can enhance the pharmacological properties of G-quadruplex-based drugs. For example, past studies have investigated the effects of introducing chemically modified base and sugar-backbone chemistries into the thrombin binding aptamer (82), known for its anti-coagulant properties. The use of modified chemistries in the thrombin binding aptamer have lead to higher stability (106,128-131,231,235), increased binding affinity (106,128), and enhanced biological activity $(122,128,231,236,237)$, including studies in vivo. In a similar manner, studies involving modified nucleic acid chemistries have enhanced the properties of anti-HIV aptamers (106,123,230,238-241). 
A

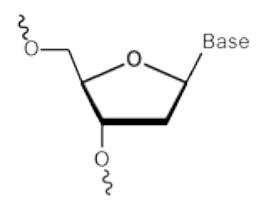

DNA

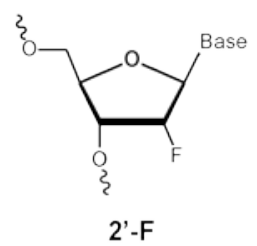

$2^{\prime}-\mathrm{F}$

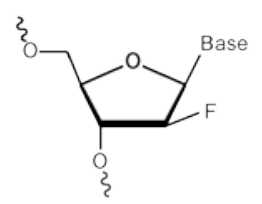

2'-F-ANA

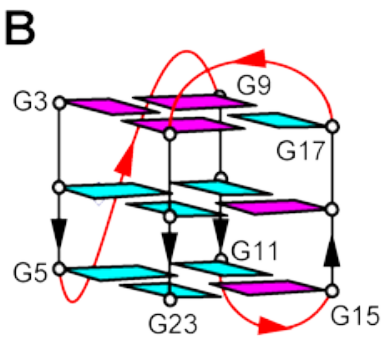

C

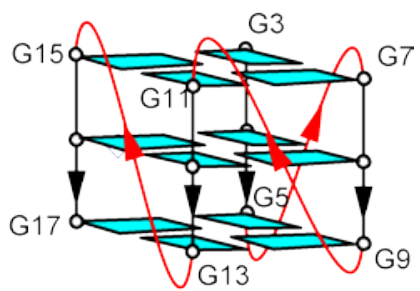

Figure 5-1. Modified nucleosides and G-quadruplex scaffolds: (A) Sugar chemistry of DNA, 2'F-ribose, and 2'-F-ANA-ribose. (B) Hybrid (3+1) G-quadruplex, formed by the "HT-series" sequence $\mathrm{d}\left[\mathrm{T}_{2}\left(\mathrm{G}_{3} \mathrm{~T}_{2} \mathrm{~A}\right)_{3} \mathrm{G}_{3} \mathrm{~A}\right]$. (C) Parallel (4+0) G-quadruplex, formed by the "PS-series" sequence $\mathrm{d}\left[\mathrm{T}_{2}\left(\mathrm{G}_{3} \mathrm{~T}\right)_{4}\right]$. Guanine bases are colored according to their syn (magenta) or anti (cyan) glycosidic conformations.

Two promising alternative nucleosides are 2'-deoxy-2'-fluoro-riboguanosine ( $\left.{ }^{\mathrm{F}} \mathrm{G}\right)$ and 2'deoxy-2'-fluoro-arabinoguanosine $\left({ }^{\mathrm{FANA}} \mathrm{G}\right)$ which contain a fluorine atom substitution at the C2' position of the sugar (Fig. 5-1). These chemistries have shown promise for increasing the stability and anti-sense potency of duplexes formed by short interference RNAs (242,243). Past studies have observed that 2'-deoxy-2'-fluoro-ribo- (2'-F) and 2'deoxy-2'-fluoro-arabino- (2'-F-ANA) nucleosides can increase the resistance of modified oligonucleotides to degradation by nuclease $(103,105,106)$. In the context of Gquadruplex, modification with 2'-F-ANA nucleosides has also allowed for enhanced Gquadruplex aptamer stability and nuclease resistance (106). Considering these beneficial properties, 2'-F and 2'-F-ANA modified nucleosides are attractive tools for use in the design and optimization of G-quadruplex based aptamers.

The goal of this study is to understand how to effectively incorporate ${ }^{\mathrm{F}} \mathrm{G}$ and ${ }^{\text {FANA }} \mathrm{G}$ into G-quadruplex DNA by examining the effects of modifications on G-quadruplex 
conformation and thermal stability. We characterized the effects of single-position substitutions of ${ }^{\mathrm{F}} \mathrm{G}$ and ${ }^{\mathrm{FANA}} \mathrm{G}$ into two G-quadruplex scaffolds. 1) The first is the intramolecular $(3+1)$ hybrid G-quadruplex formed by the human telomeric sequence $d\left[T_{2}\left(G_{3} T_{2} A\right)_{3} G_{3} A\right]$ of the "HT-series" (28). This structure contains three strands oriented in one direction with one strand running in the opposite direction (Fig 5-1B). This strand arrangement is connected by edgewise and propeller loops three nucleotides (3-nt) in length. Furthermore, guanine bases within the G-tetrad core adopt a mixture of syn and anti glycosidic conformations. 2) The second scaffold is an intramolecular parallelstranded (4+0) G-quadruplex formed by the "PS-series" sequence $d\left[T_{2}\left(G_{3} T\right)_{4}\right]$ (182). This structure contains all strands pointed in the same direction connected by short 1-nt loops, with all guanines adopting an anti conformation. These two G-quadruplex scaffolds were chosen for study as they are structurally distinct, well characterized, and demonstrate a well-defined major conformation. We evaluate the G-quadruplex conformation formed by modified sequences along with their thermal stability using a combination of nuclear magnetic resonance (NMR), ultraviolent (UV) absorption and circular dichroism (CD) spectroscopic methods (Appendix II - Protocols - Chapter 5). By testing the effects of ${ }^{\mathrm{F}} \mathrm{G}$ and ${ }^{\text {FANA }}$ G substitutions into a variety of G-quadruplex structural environments, we aim to broadly investigate how to effectively incorporate these modified nucleosides into Gquadruplex structure. Such knowledge will enable the rational design of engineered Gquadruplexes containing ${ }^{\mathrm{F}} \mathrm{G}$ and ${ }^{\mathrm{FANA}} \mathrm{G}$ chemistries. 


\subsection{Results and Discussion:}

\subsection{1: 2'-F-guanosine and 2'-F-ANA-guanosine substitutions} destabilize syn positions and are universally tolerated in anti positions

Substitutions of ${ }^{\mathrm{F}} \mathrm{G}$ and ${ }^{\mathrm{FANA}} \mathrm{G}$ were made in a systematic manner into the $(3+1) \mathrm{G}$ quadruplex scaffold of the HT-series (Table 5-1). The modifications of guanines that adopt a syn conformation in the native $(3+1)$ G-quadruplex yielded sequences that demonstrated multiple sets of imino proton peaks in NMR spectra (Fig 5-2). Additionally, these sequences generally demonstrated notable changes in CD spectra when compared with the unmodified sequence, with a decrease in signal at $295 \mathrm{~nm}$ and an increase at 260 $\mathrm{nm}$ being observed for most sequences (Fig 5-2). Collectively, this data suggests that ${ }^{\mathrm{F}} \mathrm{G}$

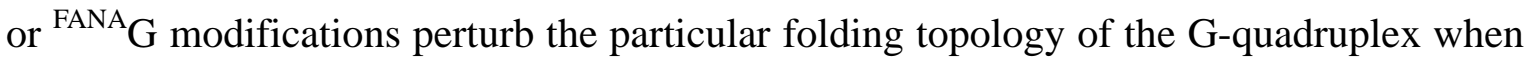
substituted into syn-position guanines.

Alternatively, ${ }^{\mathrm{F}} \mathrm{G}$ and ${ }^{\text {FANA }} \mathrm{G}$ substitutions were well tolerated when substituted into anti

A

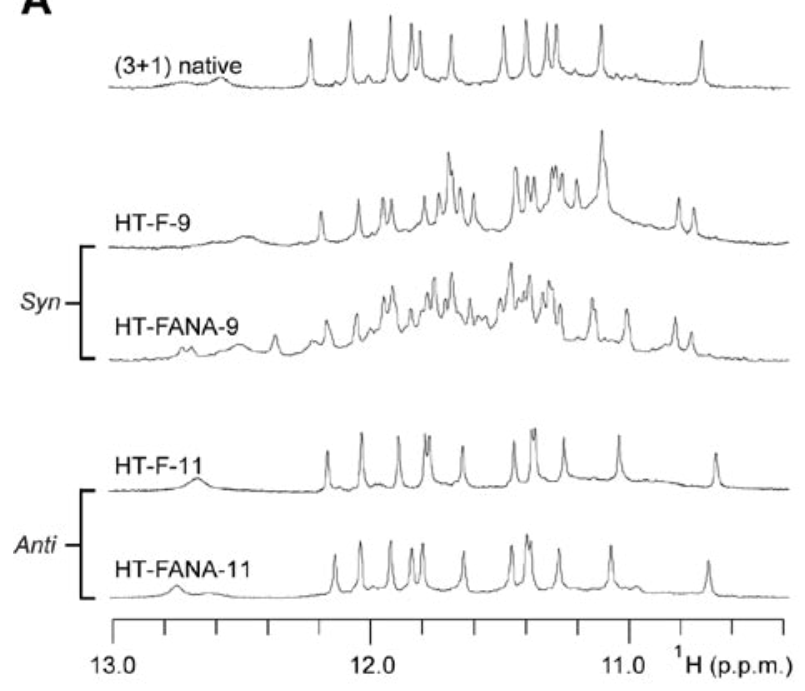

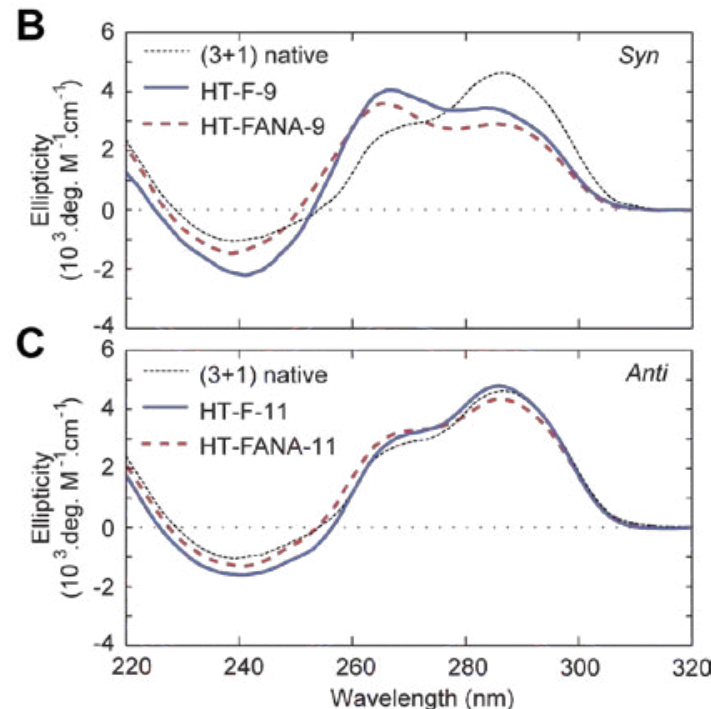

Figure 5-2. Effect of glycosidic conformation on ${ }^{\mathrm{F}} \mathrm{G}$ and ${ }^{\mathrm{FANA}} \mathrm{G}$ substitutions into the (3+1) G-quadruplex: (A) Illustrative NMR spectra of the " $(3+1)$ native" sequence and sequences containing ${ }^{\mathrm{F}} \mathrm{G} /{ }^{\mathrm{FANA}} \mathrm{G}$ substitutions to syn and anti positions. CD spectra are shown for ${ }^{\mathrm{F}} \mathrm{G} /{ }^{\mathrm{FANA}} \mathrm{G}$ substitutions to (B) syn guanine 9 and (C) anti guanine 11. 
Table 5-1. DNA Sequences and Melting Temperature Data

\begin{tabular}{|c|c|c|c|}
\hline \multirow{2}{*}{ Name $^{a}$} & \multirow{2}{*}{ Sequence $\left(5^{\prime} \rightarrow 3^{\prime}\right)^{\mathrm{b}}$} & \multicolumn{2}{|c|}{$\left({ }^{\circ} \mathrm{C}\right)$} \\
\hline & & $\mathrm{T}_{\mathrm{m}}{ }^{\mathrm{c}}$ & $\Delta \mathrm{T}_{\mathrm{m}}$ \\
\hline$(3+1)$ native & TT GGG TTA GGG TTA GGG TTA GGG A & $50.4 \pm 0.2$ & - \\
\hline HT-F-3 & TT FGG TTA GGG TTA GGG TTA GGG A & - & - \\
\hline HT-F-4 & TT GFG TTA GGG TTA GGG TTA GGG A & $50.6 \pm 0.4$ & 0.2 \\
\hline HT-F-5 & TT GGF TTA GGG TTA GGG TTA GGG A & $50.2 \pm 0.3$ & -0.2 \\
\hline HT-F-9 & TT GGG TTA FGG TTA GGG TTA GGG A & - & - \\
\hline HT-F-10 & TT GGG TTA GFG TTA GGG TTA GGG A & $47.9 \pm 1.3$ & -2.5 \\
\hline HT-F-11 & TT GGG TTA GGF TTA GGG TTA GGG A & $48.8 \pm 0.8$ & -1.5 \\
\hline HT-F-15 & TT GGG TTA GGG TTA FGG TTA GGG A & - & - \\
\hline HT-F-16 & TT GGG TTA GGG TTA GFG TTA GGG A & - & - \\
\hline HT-F-17 & TT GGG TTA GGG TTA GGF TTA GGG A & $47.7 \pm 0.5$ & -2.6 \\
\hline HT-F-21 & TT GGG TTA GGG TTA GGG TTA FGG A & - & - \\
\hline HT-F-22 & TT GGG TTA GGG TTA GGG TTA GFG A & $48.8 \pm 0.8$ & -1.6 \\
\hline HT-F-23 & TT GGG TTA GGG TTA GGG TTA GGF A & $48.5 \pm 0.1$ & -1.8 \\
\hline HT-FANA-3 & 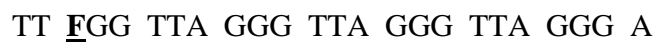 & - & - \\
\hline HT-FANA-4 & 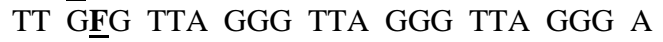 & $53.4 \pm 0.3$ & 3.1 \\
\hline HT-FANA-5 & TT GĞE TTA GGG TTA GGG TTA GGG A & $50.8 \pm 0.6$ & 0.5 \\
\hline HT-FANA-9 & TT GGG TTA $\underline{\mathbf{F}} \overline{\mathrm{GG}}$ TTA GGG TTA GGG A & - & - \\
\hline HT-FANA-10 & 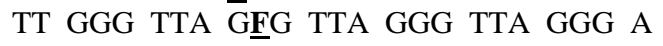 & $53.5 \pm 0.7$ & 3.1 \\
\hline HT-FANA-11 & TT GGG TTA GGEF TTA GGG TTA GGG A & $51.8 \pm 0.5$ & 1.5 \\
\hline HT-FANA-15 & 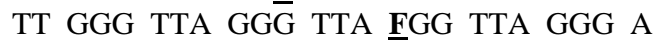 & - & - \\
\hline HT-FANA-16 & 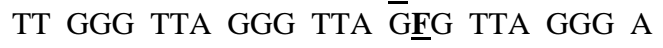 & - & - \\
\hline HT-FANA-17 & TT GGG TTA GGG TTA GḠF TTA GGG A & $52.8 \pm 0.1$ & 2.4 \\
\hline HT-FANA-21 & TT GGG TTA GGG TTA GGG TTA $\underline{\mathbf{F} G G ~ A}$ & - & - \\
\hline HT-FANA-22 & TT GGG TTA GGG TTA GGG TTA $\bar{G} \underline{F} G$ A & $52.5 \pm 0.7$ & 2.2 \\
\hline HT-FANA-23 & TT GGG TTA GGG TTA GGG TTA GḠ $\underline{\mathbf{F}}$ A & $50.9 \pm 0.5$ & 0.5 \\
\hline$(4+0)$ native & TT GGG $\mathrm{T}$ GGG $\mathrm{T}$ GGG $\mathrm{T}$ GGG $\mathrm{T}$ & $74.9 \pm 0.8$ & - \\
\hline PS-F-3 & TT FGG $\mathrm{T}$ GGG $\mathrm{T}$ GGG $\mathrm{T}$ GGG $\mathrm{T}$ & $75.6 \pm 0.1$ & 0.7 \\
\hline PS-F-4 & TT GFG T GGG T GGG T GGG $\mathrm{T}$ & $5.8 \pm 0.0$ & 0.8 \\
\hline PS-F-5 & TT GGF $\mathrm{T}$ GGG $\mathrm{T}$ GGG $\mathrm{T}$ GGG $\mathrm{T}$ & $74.2 \pm 0.1$ & -0.7 \\
\hline PS-F-8 & TT GGG $\mathrm{T}$ GFG $\mathrm{T}$ GGG $\mathrm{T}$ GGG $\mathrm{T}$ & $75.7 \pm 0.1$ & 0.7 \\
\hline PS-F-11 & TT GGG $\mathrm{T}$ GGG $\mathrm{T}$ FGG $\mathrm{T}$ GGG $\mathrm{T}$ & $76.0 \pm 0.2$ & 1.1 \\
\hline PS-F-12 & TT GGG $\mathrm{T}$ GGG $\mathrm{T}$ GFG $\mathrm{T}$ GGG $\mathrm{T}$ & $74.3 \pm 0.1$ & -0.6 \\
\hline PS-F-13 & TT GGG $\mathrm{T}$ GGG $\mathrm{T}$ GGF $\mathrm{T}$ GGG $\mathrm{T}$ & $75.4 \pm 0.3$ & 0.5 \\
\hline PS-F-17 & TT GGG $\mathrm{T}$ GGG $\mathrm{T}$ GGG $\mathrm{T}$ GGF $\mathrm{T}$ & $74.0 \pm 0.0$ & -1.0 \\
\hline PS- FANA-3 & 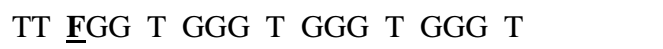 & $75.9 \pm 0.2$ & 1.0 \\
\hline PS-FANA-4 & TT $\bar{G} \underline{F G} T$ GGG $\mathrm{T}$ GGG $\mathrm{T}$ GGG $\mathrm{T}$ & $78.5 \pm 0.0$ & 3.6 \\
\hline PS-FANA-5 & TT GGE $\underline{\mathbf{F}}$ T GGG T GGG T GGG $\mathrm{T}$ & $75.9 \pm 0.3$ & 1.0 \\
\hline PS-FANA-8 & 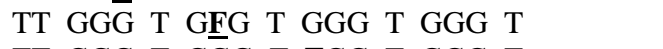 & $78.5 \pm 0.0$ & 3.6 \\
\hline PS-FANA-11 & TT GGG $\mathrm{T}$ GGG $\mathrm{T}$ FGG $\mathrm{T}$ GGG $\mathrm{T}$ & $75.9 \pm 0.2$ & 1.0 \\
\hline PS-FANA-12 & TT GGG $\mathrm{T}$ GGG $\mathrm{T}$ GEG $\mathrm{T}$ GGG $\mathrm{T}$ & $78.0 \pm 0.0$ & 3.1 \\
\hline PS-FANA-13 & TT GGG T GGG T GGEF T GGG $\mathrm{T}$ & $75.4 \pm 0.3$ & 0.5 \\
\hline PS-FANA-17 & TT GGG T GGG T GGG $\bar{T}$ GGE $\mathrm{T}$ & $75.1 \pm 0.2$ & 0.2 \\
\hline
\end{tabular}

position guanines within the $(3+1) \mathrm{G}$-quadruplex. Sequences containing modifications to anti-position guanines were observed to form a single conformation in NMR spectra with chemical shift patterns that were highly similar to that of the unmodified sequence. CD 
spectra of these sequences were also similar to that of the unmodified sequence, suggesting that modified sequences maintain the same (3+1) G-quadruplex conformation upon substitution of ${ }^{\mathrm{F}} \mathrm{G}$ or ${ }^{\mathrm{FANA}} \mathrm{G}$ (Fig 5-2). Additionally, substitutions were made into the (4+0) G-quadruplex which contains all guanines in an anti conformation. Within the (4+0) G-quadruplex, substitutions were made into 8 select positions to include a variety of structural environments including G3-G5, G8, G11-G13, and G17. All of the ${ }^{\mathrm{F}} \mathrm{G}$ and ${ }^{\text {FANA }} \mathrm{G}$ modifications into the $(4+0) \mathrm{G}$-quadruplex demonstrated highly similar NMR and CD spectra compared to the unmodified sequence with no sign of disruption to the major folding topology adopted (Fig 5-3). Collectively, our results from ${ }^{\mathrm{F}} \mathrm{G}$ and ${ }^{\mathrm{FANA}} \mathrm{G}$ substitutions into the $(3+1)$ and $(4+0)$ G-quadruplex scaffolds suggest that these chemistries are well-suited for substitution into anti-conformation guanines in variety of structural environment.

This study reports that modification of ${ }^{\mathrm{F}} \mathrm{G}$ or ${ }^{\mathrm{FANA}} \mathrm{G}$ to syn-position guanines broadly pert-
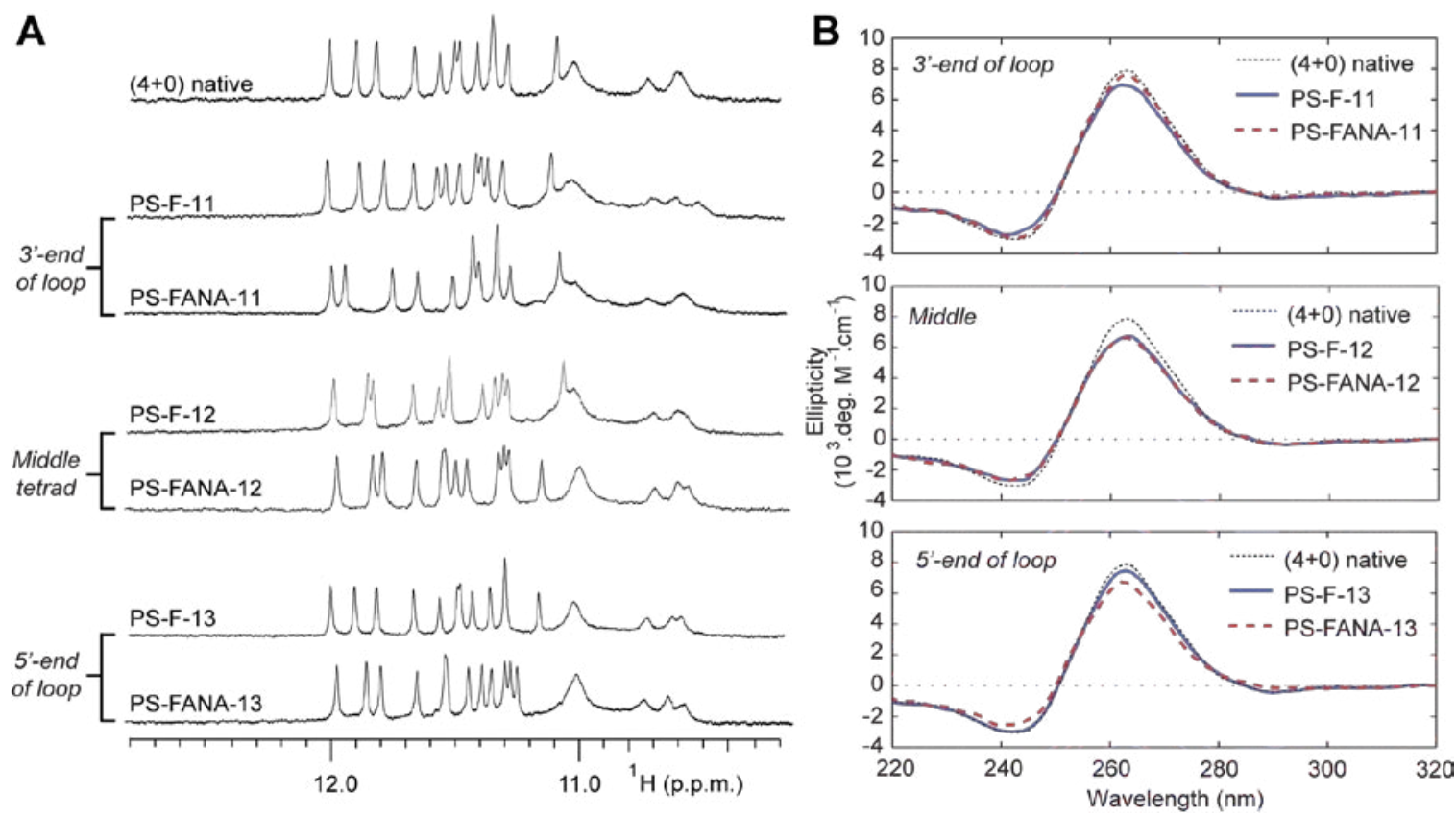

Figure 5-3. Incorporation of ${ }^{\mathrm{F}} \mathrm{G}$ and ${ }^{\mathrm{FANA}} \mathrm{G}$ substitutions into the (4+0) G-quadruplex scaffold: (A) NMR and (B) CD spectra of select PS-series samples containing single ${ }^{\mathrm{F}} \mathrm{G}$ and ${ }^{\text {FANA }} \mathrm{G}$ substitutions to guanines located in a variety of structural environments. ${ }^{\mathrm{F}} \mathrm{G}$ and ${ }^{\mathrm{FANA}} \mathrm{G}$ are well tolerated in all positions of the PSseries. 
urbs the folding topology of the $(3+1) \mathrm{G}$-quadruplex. Previous studies have shown that

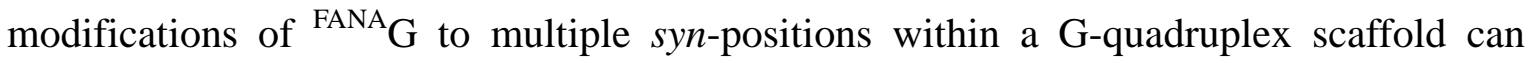
drive conformational changes (106). Others have expanded upon this to shown that rationally-placed ${ }^{\mathrm{FANA}} \mathrm{G}$ modifications can alter structural equilibrium (244). In the current work, we demonstrate that a single substitution of either ${ }^{\mathrm{F}} \mathrm{G}$ or ${ }^{\text {FANA }} \mathrm{G}$ into any of the syn-positions tested is powerful enough to induce a mixture of conformers and perturb the native (3+1) G-quadruplex folding topology. These findings suggest the general syndestabilization properties of ${ }^{\mathrm{F}} \mathrm{G}$ or ${ }^{\mathrm{FANA}} \mathrm{G}$ modified nucleosides.

In contrast, anti-position substitutions of ${ }^{\mathrm{F}} \mathrm{G}$ and ${ }^{\mathrm{FANA}} \mathrm{G}$ chemistries are tolerated in both the $(3+1)$ and $(4+0)$ G-quadruplex scaffold without disrupting the folding topology. The tolerance of these chemistries in all anti positions is interesting considering the wide range of structural environments tested. ${ }^{\mathrm{F}} \mathrm{G}$ and ${ }^{\text {FANA }} \mathrm{G}$ chemistries were successfully substituted with guanines within the central tetrad layer, adjacent to short 1-nt propeller loops, and adjacent to 3-nt edgewise and propeller loops without disrupting the native Gquadruplex conformation. Our work here suggests that, as a general rule, the ${ }^{\mathrm{F}} \mathrm{G}$ and ${ }^{\text {FANA }} \mathrm{G}$ chemistries can be universally incorporated into anti-position guanines in Gquadruplex DNA.

\subsection{2: 2'-F-ANA-guanosine substitutions stabilize G-quadruplex} DNA while 2'-F-guanosine substitutions induce mixed effects on stability

The effect of single-position ${ }^{\mathrm{F}} \mathrm{G}$ and ${ }^{\text {FANA }} \mathrm{G}$ substitutions on the melting temperature $\left(\mathrm{T}_{\mathrm{m}}\right)$ of G-quadruplexes was determined through thermal denaturing experiments employing UV absorbance spectroscopy (Fig 5-4 and Table 5-1). The $T_{m}$ values of select sequences 
were also confirmed using CD spectroscopy. Samples from the HT-series were analyzed in $5 \mathrm{mM} \mathrm{KCl}$ and $5 \mathrm{mM} \mathrm{KPi}$ salt conditions while samples from the PS-series were analyzed in $1 \mathrm{mM} \mathrm{KPi}$. Sequences containing single-position substitutions into antiposition guanines were analyzed. These sequences were chosen for analysis as they adopt the same conformation as their parent sequences, a necessary prerequisite to a meaningful quantitative comparison of thermal stability.

${ }^{\text {FANA }} \mathrm{G}$ substitutions were observed to moderately stabilize the $(3+1)$ G-quadruplex scaffold with $\Delta \mathrm{T}_{\mathrm{m}}$ values in the range of $+0.5^{\circ} \mathrm{C}$ to $+3.1^{\circ} \mathrm{C}$ (Fig. $5-4 \mathrm{~A}$ ). Within the (4+0) G-quadruplex scaffold, ${ }^{\text {FANA }} \mathrm{G}$ substitutions were also stabilizing with $\Delta \mathrm{T}_{\mathrm{m}}$ values in the range of $+0.2^{\circ} \mathrm{C}$ to $+3.6^{\circ} \mathrm{C}$ (Fig. $5-4 \mathrm{~B}$ ). Substitutions of ${ }^{\text {FANA }} \mathrm{G}$ into central tetrad layers in both scaffolds were observed to be particularly stabilizing, with $\Delta \mathrm{T}_{\mathrm{m}}$ values of $+2.2^{\circ} \mathrm{C}$ to $+3.1^{\circ} \mathrm{C}$ observed in the HT-series and values of $+3.1^{\circ} \mathrm{C}$ to $+3.6^{\circ} \mathrm{C}$ observed for the PSseries. On the contrary, sequences containing ${ }^{\mathrm{F}} \mathrm{G}$ substitutions were generally mildly dest-
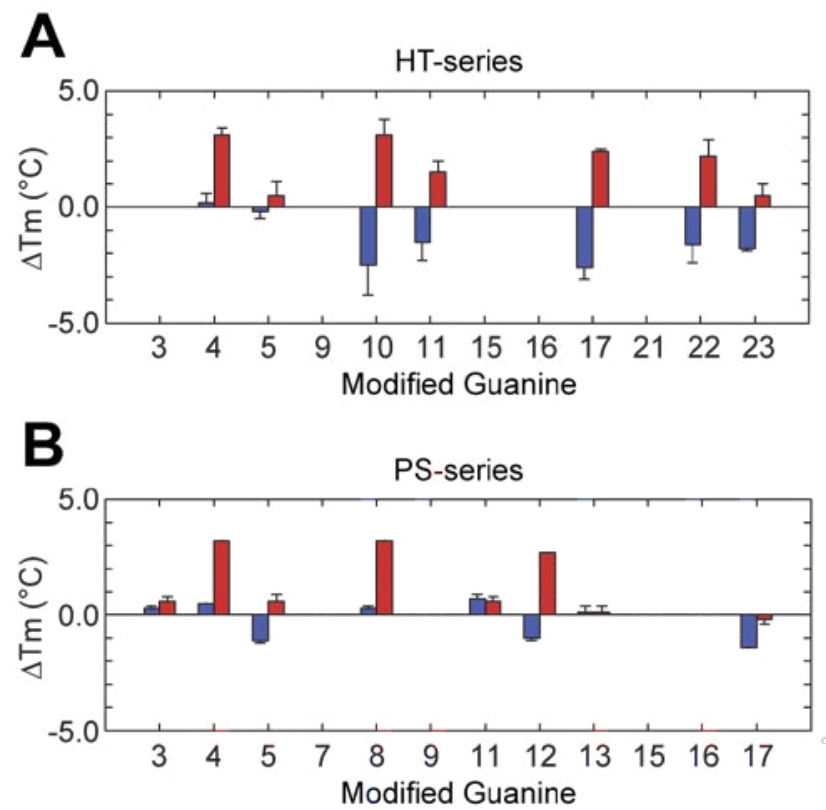

Figure 5-4. Change in the melting temperature for single position ${ }^{\mathrm{F}} \mathrm{G}$ (blue) and ${ }^{\text {FANA }} \mathrm{G}$ (red) substitutions to (A) anti positions in the $(3+1)$ G-quadruplex of the HT-series and (B) the (4+0) G-quadruplex of the PS-series. Error bars indicate the hysteresis between heating and cooling melting curves. 
abilized in the HT-series with $\Delta \mathrm{T}_{\mathrm{m}}$ values between $-2.6^{\circ} \mathrm{C}$ and $+0.2^{\circ} \mathrm{C}$. ${ }^{\mathrm{F}} \mathrm{G}$ substitutions were slightly more favorable in the PS-series with $\Delta \mathrm{T}_{\mathrm{m}}$ values between $-0.7^{\circ} \mathrm{C}$ and $+1.1^{\circ} \mathrm{C}$. No clear tetrad-layer preference is observed for ${ }^{\mathrm{F}} \mathrm{G}$ substitutions.

The results of our thermal stability studies indicate that ${ }^{\text {FANA }} \mathrm{G}$ modifications are moderately stabilizing. ${ }^{\mathrm{F}} \mathrm{G}$ substitutions are observed to be tolerated within the two scaffolds, generally destabilizing the $(3+1)$ G-quadruplex by $1-2^{\circ} \mathrm{C}$ while having minor and mixed effects on the stability of the (4+0) G-quadruplex. In all anti positions tested over both scaffolds, ${ }^{\text {FANA }} \mathrm{G}$ is observed to be of equal or greater effectiveness in stabilizing G-quadruplex structure compared to its ${ }^{\mathrm{F}} \mathrm{G}$ counterpart. This observation is in agreement with recent work describing ${ }^{\text {FANA }} \mathrm{G}$ to be a more powerful substituent than ${ }^{\mathrm{F}} \mathrm{G}$ for driving structural equilibrium (244). Interestingly, substitutions of ${ }^{\text {FANA }} \mathrm{G}$ to guanosines in the central G-tetrad of the 3-layered (3+1) and (4+0) G-quadruplexes were found to be most stabilizing compared to other positions (Fig 5-4).

\subsection{3: ${ }^{\mathrm{F}} \mathrm{G}$ and ${ }^{\mathrm{FANA}} \mathrm{G}$ are powerful tools for structural manipulation of G-quadruplex}

Alternative nucleic acids can be used to manipulate G-quadruplex structure. As the conformation of the guanine base about the glycosidic bond is intrinsically related to the folding topology of G-quadruplexes (67), one approach is to use modified nucleotides that favour a syn or anti guanine base conformation $(25,125,129,229)$. For example, substitutions of a guanine with C8-modified guanine bases, such as 8-bromoguanine $(25,32)$, is one technique for manipulating structure, as these modifications favor substitution into syn positions (113). Considering this, we sought to explore the ability of ${ }^{\mathrm{F}} \mathrm{G}$ and ${ }^{\mathrm{FANA}} \mathrm{G}$ to manipulate folds adopted by G-quadruplex forming sequences. 

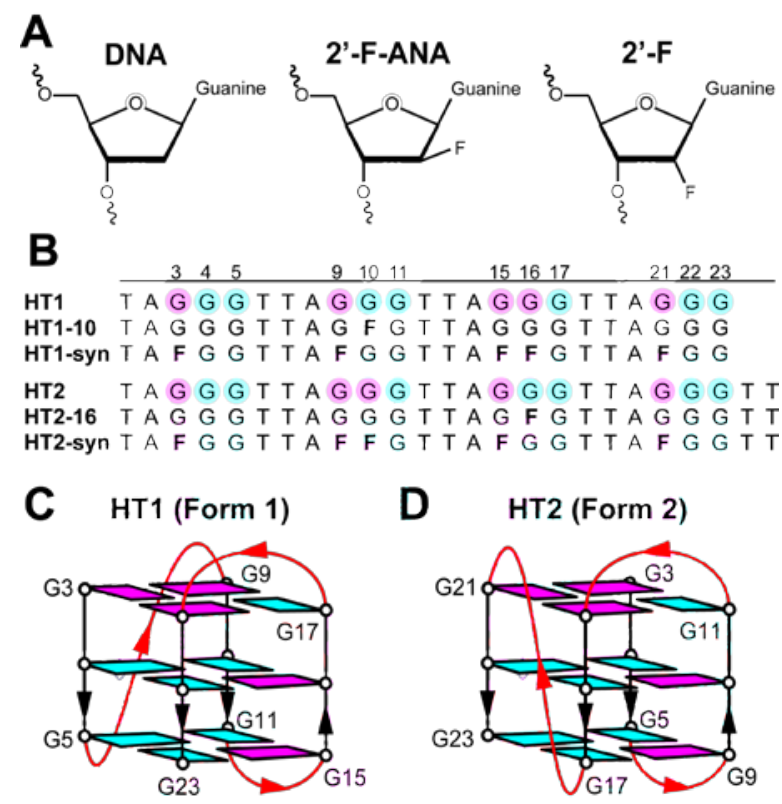

Figure 5-5: (A) Fig. 1 (A) Sugar schematic of DNA, 2'-F-ANA, and 2'-F chemistries. (B) Sequences explored in this work. " $F$ '" denotes a ${ }^{\text {FANA }} \mathrm{G}$ or ${ }^{\mathrm{F}} \mathrm{G}$ modification. The HT1-syn and HT2-syn nomenclature signifies substitution to all guanines that adopt syn conformations in the HT1 or HT2 sequences. (C,D) The major form $(3+1)$ hybrid Gquadruplex folding topologies of the (C) HT1 and (D) HT2 sequences. Syn and anti guanine glycosidic conformations are shown in magenta and cyan respectively.

First, We examine the effectiveness of ${ }^{\text {FANA }} \mathrm{G}$ and ${ }^{\mathrm{F}} \mathrm{G}$ in decreasing structural polymorphism when substituted within sequences that adopt multiple G-quadruplex forms in solution. Two G-quadruplex forming human telomeric sequences are examined, both of which demonstrate a $(3+1)$ hybrid folding topology along with minor conformations (27,28). The sequence $d\left[T A\left(G_{3} T_{2} A\right) G_{3}\right]$, termed HT1, exists predominantly in Form 1, (28) (Fig 5-5C) while d[TA( $\left.\left(\mathrm{G}_{3} \mathrm{~T}_{2} \mathrm{~A}\right) \mathrm{G}_{3} \mathrm{~T}_{2}\right]$, termed HT2, has a major fold of Form 2 (27) (Fig 5-5D). The folding topology of G-quadruplexes is very sensitive to sequence changes, even amongst similar sequences $(67,232)$. Although the HT1 and HT2 sequences differ only by the presence of two extra thymine bases at the 3' end of HT2, they adopt different major folding topologies. While both sequences form a $(3+1)$ hybrid folding topology, the two structures are uniquely different. The loop arrangement of Form 1 (Fig 5-5C) (propeller $\rightarrow$ edge-wise $\rightarrow$ edge-wise) is quite 
different from that of Form 2 (Fig 5-5D) (edge-wise $\rightarrow$ edge-wise $\rightarrow$ propeller), resulting in different glycosidic conformations of guanine bases G10 and G16 (Fig 5-5B).

We use rational substitutions of a single ${ }^{\text {FANA }} \mathrm{G}$ or ${ }^{\mathrm{F}} \mathrm{G}$ chemistry in order to further favor the major conformation in HT1 and HT2 as our studies above indicate their anti-favoring capabilities. To accomplish this, we substitute the anti-favoring ${ }^{\text {FANA }} \mathrm{G}$ and ${ }^{\mathrm{F}} \mathrm{G}$ nucleotides into anti-position guanines in one form that adopt a syn conformation in the other form. As positions 10 and 16 adopt different glycosidic conformations in the hybrid (3+1) Form 1 and Form 2, substitutions were made to HT1 in the position that adopts anti in Form 1 and syn in Form 2, and vice versa (Fig 5-5B). It was hypothesized that a substitution at these positions should stabilize the desired form while potentially destabilizing undesired forms.

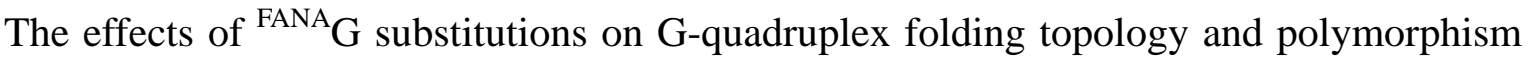
were examined by CD and ${ }^{1} \mathrm{H}$ NMR spectroscopy (Fig 5-6). As predicted, imino proton spectra reveal the reduction of minor conformations, favoring a single conformer. The high similarity between the major form in unmodified and modified NMR spectra strong-

A

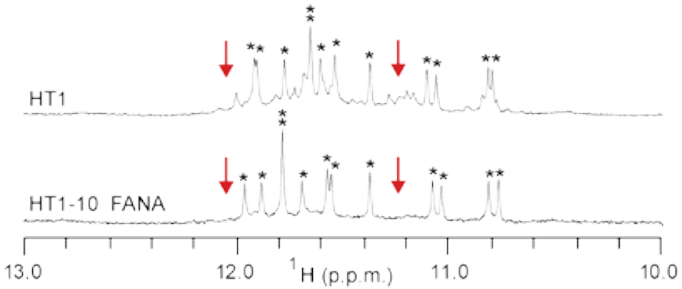

C

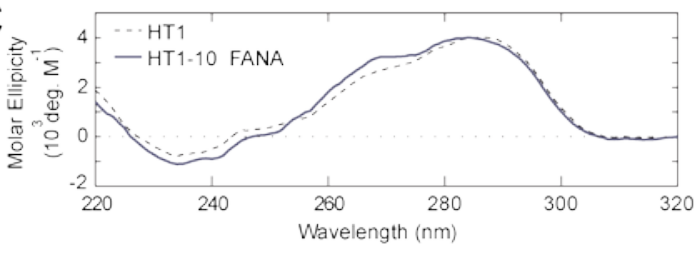

B

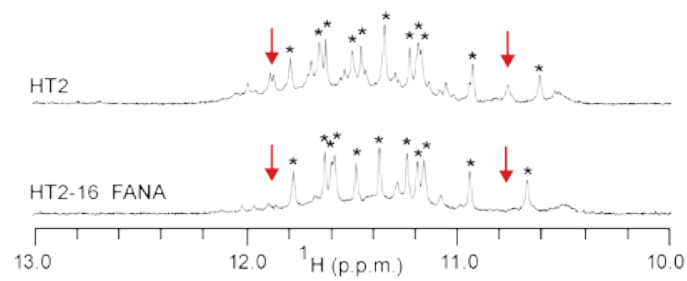

D

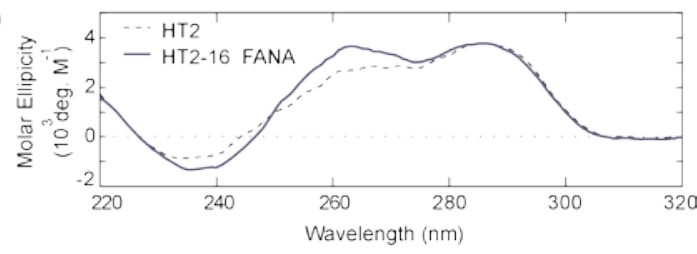

Figure 5-6: Decreasing polymorphism: (A,B) NMR imino proton and (C,D) CD spectra of (A,C) Form 1 and (B,D) Form 2 unmodified and ${ }^{\text {FANA }} \mathrm{G}$ modified sequences. Proton peaks of the major form are indicated by asterisks $(*)$. Arrows highlight the reduction of minor conformations in modified sequences. 
Table 5-2. Thermal stability analysis

\begin{tabular}{lcc}
\hline \multicolumn{1}{c}{ Sequence name } & $\mathrm{T}_{\mathrm{m}}\left({ }^{\circ} \mathrm{C}\right)$ & $\Delta \mathrm{T}_{\mathrm{m}}\left({ }^{\circ} \mathrm{C}\right)$ \\
\hline HT1 & 61.1 & - \\
HT1-10 FANA & 64.6 & +3.4 \\
HT1-syn FANA & 71.4 & - \\
& & \\
HT2 & 58.7 & - \\
HT2-16 FANA & 62.0 & +3.3 \\
HT2-syn FANA & 69.9 & - \\
\hline
\end{tabular}

ly suggests the same major fold is adopted (Fig 5-6). CD spectra also support that $(3+1)$ hybrid folding topologies are adopted. UV melting experiments were performed to analyze the thermal stability of modified sequences (Table 5-2). Data indicate that modified sequences containing a single ${ }^{\text {FANA }}$ G substitution maintain stable G-quadruplex structures upon modification. The effects of modifications by ${ }^{\mathrm{F}} \mathrm{G}$ on NMR imino proton spectra were also examined (Fig 5-7). ${ }^{\mathrm{F}} \mathrm{G}$ is observed to be less effective at decreasing the minor conformations present in HT1 and HT2 compared to ${ }^{\text {FANA }}$ G. Collectively, these results indicate that a single ${ }^{\text {FANA }} \mathrm{G}$ substitution is powerful enough to favor a single conformation from polymorphic sequences. The approach presented here has general application towards facilitating the structural studies of polymorphic G-quadruplexes sequences $(67,121)$.

In addition to ${ }^{\text {FANA }} \mathrm{G}$ being a powerful tool in decreasing polymorphism, 2'-F-modified nucleotides such as ${ }^{\text {FANA }} \mathrm{G}$ and ${ }^{\mathrm{F}} \mathrm{G}$ have the added potential to be used as ${ }^{19} \mathrm{~F}$ NMR struct-
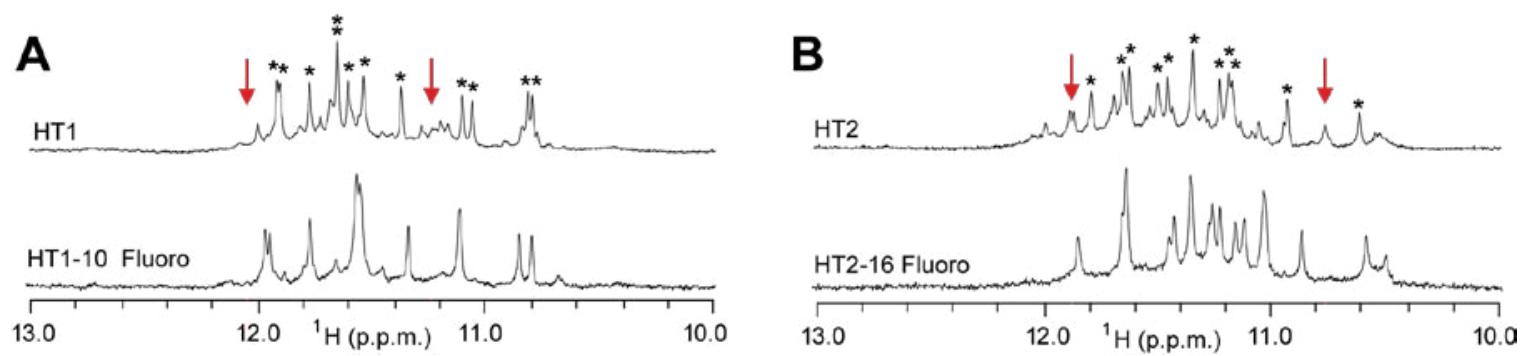

Figure 5-7: Decreasing polymorphism: NMR imino proton (A) Form 1 and (B) Form 2 unmodified and ${ }^{\mathrm{F}} \mathrm{G}$ modified sequences. Proton peaks of the major form are indicated by asterisks (*). Arrows highlight the presence of minor conformations in unmodified sequences. 

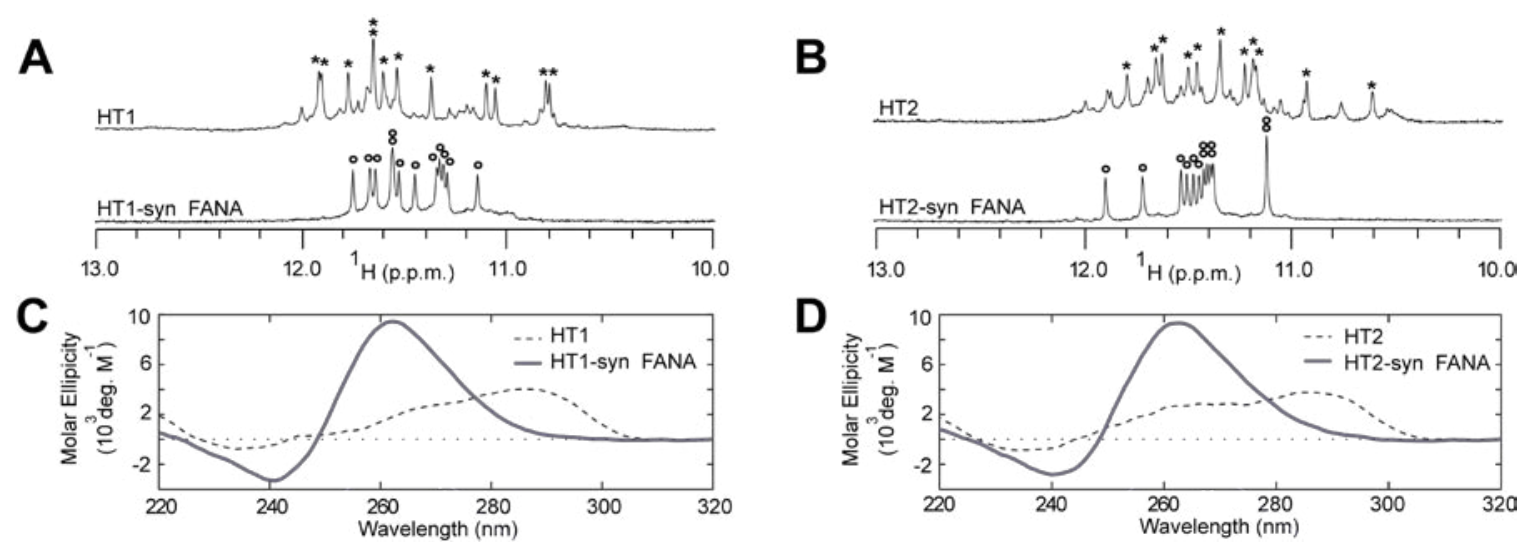

Figure 5-8: Conformational switching: (A,B) NMR imino proton and (C,D) CD spectra of (A,C) Form 1 and (B,D) Form 2 unmodified and ${ }^{\text {FANA }} \mathrm{G}$ syn-modified sequences. Proton peaks of the major form are indicated by asterisks $(*)$ in the unmodified sequences and by $\left(^{\circ}\right)$ in the syn-modified sequences.

ural probes. Rational substitution allows for ${ }^{19} \mathrm{~F}$ NMR capabilities without causing large changes in G-quadruplex structure. Such ${ }^{19} \mathrm{~F}$ NMR probes can be used to understand the number of conformations present in solution due to the simplicity of ${ }^{19} \mathrm{~F}$ spectra. Similarly, ${ }^{19} \mathrm{~F}$ NMR could be used as a probe to study the interaction of G-quadruplexes with protein or small molecules.

Secondly, we examine the ability of ${ }^{\text {FANA }} \mathrm{G}$ and ${ }^{\mathrm{F}} \mathrm{G}$ substitutions to drive conformational switching of G-quadruplex folding topology. We attempt to push structural equilibrium from the native $(3+1)$ hybrid folding of HT1 and HT2 towards a parallel-stranded conformation by rational ${ }^{\text {FANA }} \mathrm{G}$ substitutions to syn-position guanines in the major folds of these sequences. NMR imino proton spectra show 12 imino proton peaks indicating that a single conformation is formed in modified sequences (Fig. 5-8). CD spectra with a positive peak at $260 \mathrm{~nm}$ and not at $290 \mathrm{~nm}$ suggest a parallel folding topology as previously reported (121). Thermal stability analysis reveals that the structures adopted by modified sequences are highly stable (Table 5-2). Modifications with ${ }^{\mathrm{F}} \mathrm{G}$ to syn positions in a similar manner also demonstrate the capacity to drive structural switching

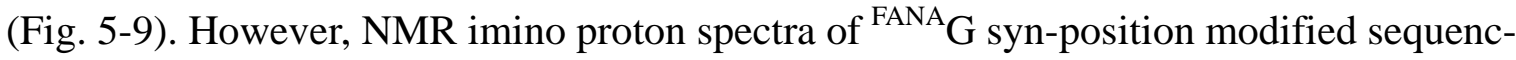



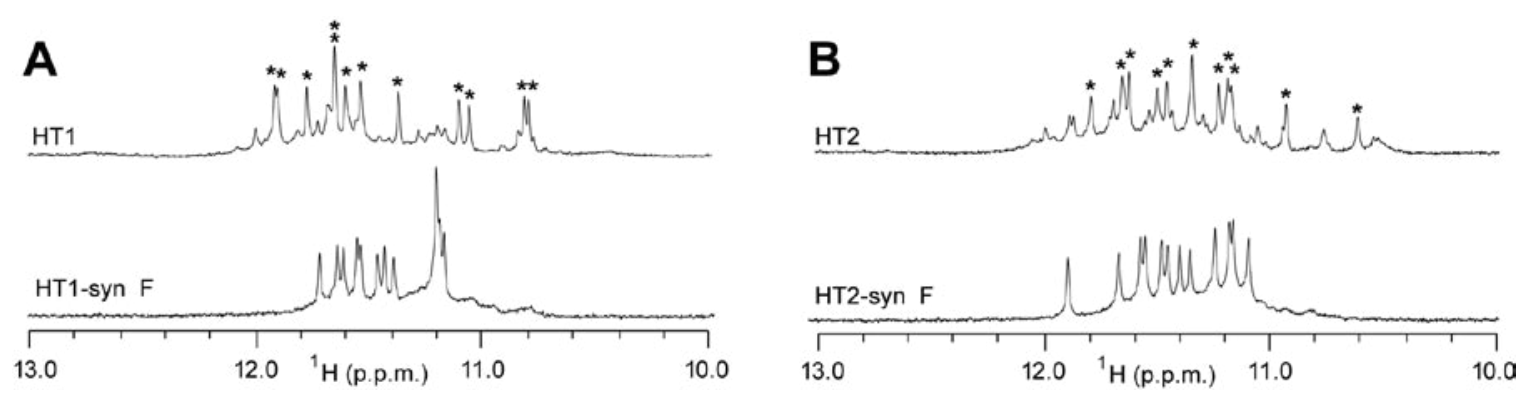

Figure 5-9: Conformational switching: NMR imino proton spectra of (A) Form 1 and (B) Form 2 unmodified and ${ }^{\mathrm{F}} \mathrm{G}$ syn-modified sequences. Proton peaks of the major form are indicated by asterisks $\left({ }^{*}\right)$ in the unmodified sequences.

es are observed to be marginally cleaner than their ${ }^{\mathrm{F}} \mathrm{G}$ counterpart. The different orientation of the 2'-fluorine atom in ${ }^{\text {FANA }} \mathrm{G}$ and ${ }^{\mathrm{F}} \mathrm{G}$ appears to influence the effectiveness of these tools. This may be due to a difference in the preferred sugar pucker of these two nucleotides or differences in the favorable intra- and inter-nucleotide fluorine-hydrogen interactions such as those previously observed in duplex DNA (245). Collectively, our data show that modifications by ${ }^{\text {FANA }} \mathrm{G}$ or ${ }^{\mathrm{F}} \mathrm{G}$ can drive a single parallel conformation from the polymorphic HT1 and HT2 human telomeric sequences.

While the first crystallographic structure of a human telomeric G-quadruplex revealed a parallel folding topology (21), obtaining a NMR solution structure has been a difficult undertaking as such a form has only observed in crowded solution (246). The ability of ${ }^{\text {FANA }} \mathrm{G}$ and ${ }^{\mathrm{F}} \mathrm{G}$ modifications to drive a single parallel conformation of human telomeric DNA in solution speaks of their effectiveness as tools for controlling the fold of Gquadruplexes. Conformational switching using modified nucleic acid chemistries has been explored in the past. Studies have driven G-quadruplex conformational changes using syn-favoring 8-bromoguanine substitutions (25,32). C8-modified nucleotides are powerful, but cannot be used to favor parallel structures or other folding topologies that lack syn positions. To compliment this tool, the development of anti-favoring tools is crucial. A study by Tang and Shafer used substitutions of ribonucleotides to drive 
conformational switching in G-quadruplexes (129). The anti-favoring locked nucleic acid, or LNA, has also been used to drive conformational changes in G-quadruplex DNA $(125,229)$. Being easier to handle than ribonucleotides, less structurally rigid than LNA, and a useful ${ }^{19} \mathrm{~F}$ NMR probe, ${ }^{\text {FANA }} \mathrm{G}$ and ${ }^{\mathrm{F}} \mathrm{G}$ represent powerful new tools in the toolbox of anti-favoring nucleotides. 


\subsection{Conclusions:}

In summary, this work examines the effects of systematic single-substitutions of ${ }^{\mathrm{F}} \mathrm{G}$ and ${ }^{\text {FANA }} \mathrm{G}$ nucleosides into a $(3+1)$ hybrid and a $(4+0)$ parallel G-quadruplex scaffold. We demonstrate the versatility of 2'-F-ANA-guanosine and 2'-F-guanosine incorporation into G-quadruplex DNA. A single modification of ${ }^{\mathrm{F}} \mathrm{G}$ or ${ }^{\mathrm{FANA}} \mathrm{G}$ to a syn guanine in the $(3+1)$ hybrid G-quadruplex perturbs this conformation. Alternatively, substitutions into all antipositions are well tolerated and do not disrupt the conformation of the $(3+1)$ or the $(4+0)$ G-quadruplexes, suggesting these nucleosides are universally well-suited for substitution into anti-conformation guanine within the G-tetrad core of G-quadruplexes. ${ }^{\text {FANA }} \mathrm{G}$ is observed to be better tolerated than ${ }^{\mathrm{F}} \mathrm{G}$ in both scaffolds, with ${ }^{\mathrm{FANA}} \mathrm{G}$ substitutions into central G-tetrad guanines being particularly stabilizing. Modification by 2'-F-ANAguanosine is demonstrated to be a powerful tool in driving a single conformation of Gquadruplex DNA from a mixture of conformers. Such modifications along with 2'-Fguanosine can be used to convert the folding topology of G-quadruplexes. These 2'-Fmodified nucleotides represent new tools in the study, design, and manipulation of Gquadruplex structure. 


\section{Chapter 6:}

\section{Engineering the Base: C8-modified Guanines}

This chapter was largely adapted from the authors work: Lech, C.J., Lim, J.K.C., Lim, J.M.W., Amrane, S., Heddi, B., Phan, A.T. (2011) Effects of Site-Specific Guanine C8-Modifications on an intramolecular DNA G-quadruplex. Biophysical Journal. 101. 1987-1996.

Author Contributions: DNA synthesis, purification and thermal denaturing experiments were carried out by S.A., J.K.C.L., J.M.W.L. and C.J.L.; Data analysis of thermal denaturing experiments was performed by C.J.L., S.A., J.K.C.L. and J.M.W.L.; Circular Dichroism studies were performed by C.J.L., S.A., J.K.C.L. and J.M.W.L.; 1D NMR experiments including N15 filtered experiments were performed by S.A., J.K.C.L. and J.M.W.L. and C.J.L.; QM Computations and data analysis were performed by C.J.L., B.H. and A.T.P.; The above mentioned manuscript was written by C.J.L, B.H. and A.T.P; All authors contributed to the conceptualization and planning of experiments.

\subsection{Introduction:}

The preferred folding topology of a G-quadruplex forming nucleic acid sequence can be altered by introducing a guanine-like aromatic base within a G-tetrad. For example, guanine derivatives such as inosine $(121,247,248)$ and 8-bromo-guanine $(113,249)$ have been used as tools in structural studies to favor a single G-quadruplex conformation from a sequence that forms multiple conformations. The guanine derivative 8-bromo-guanine belongs to a specific class of modified bases involving substitutions of the C8-hydrogen with other modification groups (Fig. 6-1). C8-modifications can be made without disrupting hydrogen bonding within the G-tetrad. This strategy was successfully used to incorporate a fluorescent probe within a G-quadruplex structure (250). In contrast to base modifications that disrupt hydrogen bonding and destabilize G-quadruplexes $(111,112)$, C8-modified derivatives 8-bromo-guanine $(113,249)$ and 8-methyl-guanine (118) have shown a tendency to increase the thermal stability of a G-quadruplex when substituted 
with guanines that originally adopt syn glycosidic conformations in the G-tetrad core. Additionally, C8-modified derivatives have been shown to influence the folding kinetics of G-quadruplex structures $(111,116,117)$.

Past research investigating the effects of C8-modifications on G-quadruplexes has been carried out on both intramolecular $(112,113,118,249,250)$ and tetramer structures (111,114-117). Intramolecular G-quadruplex scaffolds allow for single-position modifications to be implemented and to probe the effects of modifications on syn/anti glycosidic conformations. In parallel-stranded tetrameric G-quadruplexes all core guanines originally adopt anti conformations. In a tetrameric system, the substitution of a guanine by a C8-modified derivative is reflected about the entire tetrad due to the symmetry of the structure. C8-modified guanine substitutions in a tetrameric system have been reported to cause a change in the polarity of the modified tetrad $(114,115,117)$. Some studies have noted position-dependent effects for the derivatives 8-bromo-guanine (111,114), 8-methyl-guanine (115,117), and 8-amino-guanine (116), including the ability of substitutions at the 5' end to stabilize G-quadruplexes. While studies involving tetrameric G-quadruplexes are valuable, conclusions arising from these studies could be fundamentally different in comparison to those arising from intramolecular scaffolds where only one guanine within a G-tetrad is substituted at a time. With mounting evidence for the in vivo roles of G-quadruplexes, the effects of C8-modified derivatives on these structures are of physiological importance considering the numerous naturally occurring C8-modifications with suggested roles in mutagenesis including 8-oxo-guanine (251,252), 8-amino-guanine (253,254), and 8-methyl-guanine (255). From an engineering perspective, investigating the effects of various C8-modified derivatives on Gquadruplexes allows for their effective application in engineered systems to achieved desired properties. 


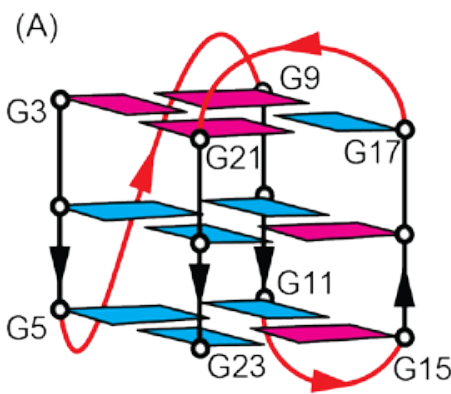

(C)<smiles></smiles>

(D)

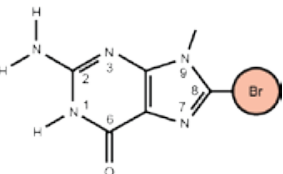

(B)

(E)<smiles></smiles>

( $\mathrm{F})$

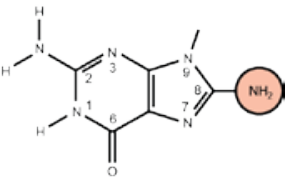

(G)

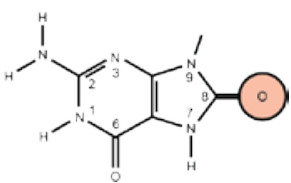

Figure 6-1: (A) Schematic structure of the $(3+1)$ G-quadruplex platform used in this study. Anti and syn guanines are shown in light gray and dark gray, respectively. (B) Chemical schematic of a modified guanine tetrad with a centrally located $\mathrm{K}^{+}$ion. The C8-modified guanine derivative is depicted by a modification group R. (C-G) The chemical schematics of guanine and the guanine derivatives explored in this work: (C) guanine, (D) 8-bromo-guanine, (E) 8-O-methyl-guanine, (F) 8-amino-guanine, and (G) 8-oxo-guanine.

In order to understand how site-specific C8-modifications of guanines affect Gquadruplex stability and structure, this work explores the effects of substituting guanine derivatives 8-bromo-guanine $\left({ }^{\mathrm{Br}} \mathrm{G}\right)$, 8-O-methyl-guanine ( $\left.{ }^{\text {Omet }} \mathrm{G}\right)$, 8-amino-guanine $\left({ }^{\mathrm{Am}} \mathrm{G}\right)$, and 8-oxo-guanine $\left({ }^{\mathrm{Oxo}} \mathrm{G}\right)$ within a well-defined intramolecular $(3+1)$ telomeric Gquadruplex structure (256) (Fig. 6-1) using a combination of UV, CD, NMR spectroscopy and $a b$ initio quantum mechanical calculations (Appendix II - Protocols - Chapter 6). The site-specific effects of substitutions to both syn and anti guanines were examined. Folding topology was monitored, allowing for a meaningful thermal denaturing analysis within the context of a single conformer. The observation of an interesting pattern in NMR chemical shift change was reproduced using $a b$ initio computational methods, providing insight into the structural effects of these modifications on G-tetrad geometry. 


\subsection{Results:}

\subsection{1: Site-specific substitutions of a guanine with an 8-bromo- guanine, 8-O-methyl-guanine, 8-amino-guanine, or 8-oxo-guanine:}

In this study, we explored the effects of base modifications on an intramolecular $(3+1) \mathrm{G}$ quadruplex structure (Fig. 6-1A) formed by the human telomeric $d\left[T_{2}\left(G_{3} T_{2} A\right)_{3} G_{3} A\right]$ sequence (256), (henceforth referred to as the unmodified sequence). Individual guanines (G) were site-specifically substituted with four different guanine derivatives $\left({ }^{\mathrm{Br}} \mathrm{G}\right.$, ${ }^{\text {Omet }} \mathrm{G}$,

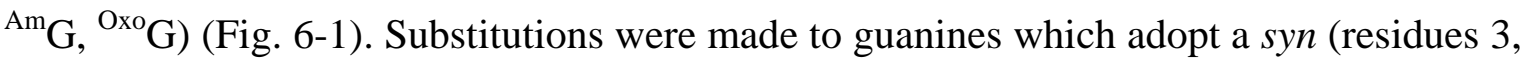
9, 15, 16 and 21) or anti (residue 22) glycosidic conformations in the original (3+1) Gquadruplex (Fig. 6-1A). Each modified sequence used in this work contained only one or two substitutions (Table 6-1).

Table 6-1: List of DNA Sequences Examined in this work

\begin{tabular}{|c|c|}
\hline Sequence Name & Sequence $\left(5^{\prime} \rightarrow 3^{\prime}\right)$ \\
\hline Unmodified & TT GGG TTA GGG TTA GGG TTA GGG A \\
\hline $\mathrm{Br}-3$ & T T B GG TTA GGG TTA G G T TA GGG A \\
\hline Br-9 & TT GGG TTA B GG TTA GGG TTA GGG A \\
\hline Br-15 & TT GGG TTA GGG TTA B G G TA GGG A \\
\hline $\mathrm{Br}-16$ & TT GGG TTA GGG TTA GBG TTA GGGA \\
\hline $\mathrm{Br}-21$ & TT GGG TTA GGG TTA GGG TTA BGGA \\
\hline $\mathrm{Br}-22$ & TT GGG TTA GGG TTA GGG TTA GBGA \\
\hline Br-9-22 & T T G G G T TA B G T TA G G T TA G B G A \\
\hline Omet-3 & T T O GG T TA GGG T TA GGG T TA G G A A \\
\hline Omet-9 & T T GGG T TA OGG T TA GGG TTA GGG A \\
\hline Omet-15 & TT GGG TTA GGG TTA OGG TTA GGG A \\
\hline Omet-16 & TT GGG TTA GGG TTA GOG TTA GGG A \\
\hline Omet-21 & TT GGG TTA GGG TTA GGG T TA OGG A \\
\hline Omet-22 & TT GGG TTA GGG TTA GGG TTA GOG A \\
\hline Br-9-Omet-22 & TTGGG TTA BGG TTA GGG TTA GOG A \\
\hline Am-3 & 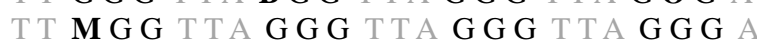 \\
\hline Am-9 & T T GGG TTA MGG TTA GGG TTA GGG A \\
\hline Am-15 & TT GGG TTA GGG TTA MGG TTA GGG A \\
\hline Am-16 & TT GGG TTA GGG TTA GMG TTA GGG A \\
\hline Am-21 & TT GGG TTA GGG TTA GGG TTA MGG A \\
\hline Am-22 & T T GGG TTA GGG TTA GGG TTA GMG A \\
\hline Br-9-Am-22 & TT GGG TTA B GG TTA G G G TA GMGA \\
\hline Oxo-3 & TT $\mathbf{X G G}$ T TA GGG T TA GGG T TA G G A \\
\hline Oxо-9 & TT GGG TTA XGG TTA GGG TTA GGG A \\
\hline Oxo-15 & TT GGG TTA GGG TTA XGG TTA GGG A \\
\hline
\end{tabular}

Modified residues are depicted as "B" for 8-bromo-guanine, "O" for 8-O-methyl-guanine, "M" for 8-aminoguanine, and " $\mathbf{X}$ " for 8-oxo-guanine. Columns of modified positions are shaded. 
Circular dichroism (CD) profiles of modified sequences containing ${ }^{\mathrm{Br}} G$, ${ }^{\text {Omet }} \mathrm{G}$, and ${ }^{\mathrm{Am}} \mathrm{G}$ modifications displayed a positive peak at $290 \mathrm{~nm}$ and a shoulder at $260 \mathrm{~nm}$, similar to the CD profile of the unmodified sequence (Fig. 6-2), consistent with the formation of a (3+1) G-quadruplex as the major form in these sequences (257).

Imino proton NMR spectra were used to further assess the formation of G-quadruplexes. In all sequences containing ${ }^{B r} G$, ${ }^{\text {Omet }} G$, and ${ }^{A m} G$, the observation of roughly twelve major imino protons at 10.5-12.5 ppm indicated the formation of a predominant G-quadruplex conformation (Fig. 6-3A-D). The similar spectral pattern between these peaks and those of the unmodified sequence strongly suggested the formation of the same $(3+1) \mathrm{G}$ quadruplex fold. Note that most changes in chemical shifts were observed only for protons from the G-tetrad containing the modified base.

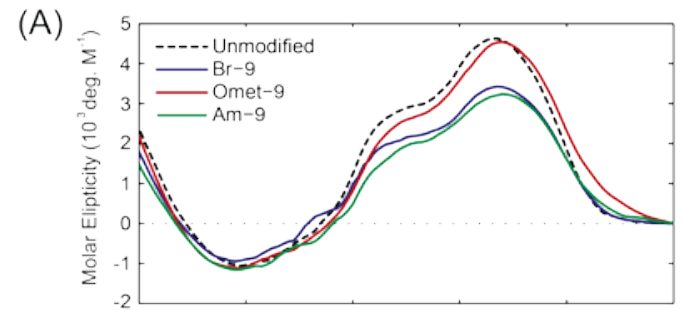

(B)

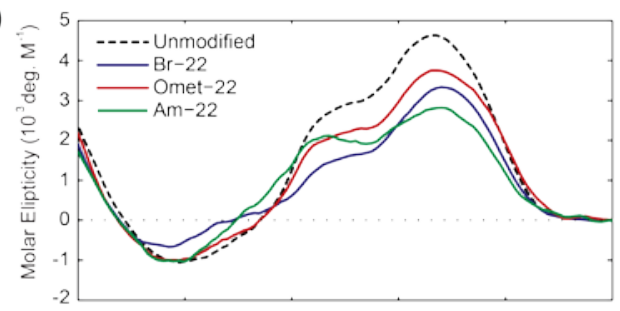

(C)

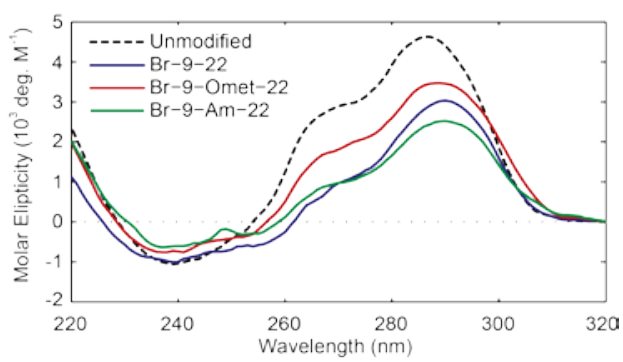

Figure 6-2: A comparison of CD spectra for sequences containing (A) single substitutions to syn residue 9, (B) single substitutions to anti residue 22, and (C) double substitutions to both syn residue 9 and anti residue 22. The CD spectrum of the unmodified sequence (dotted line) is shown as a reference 


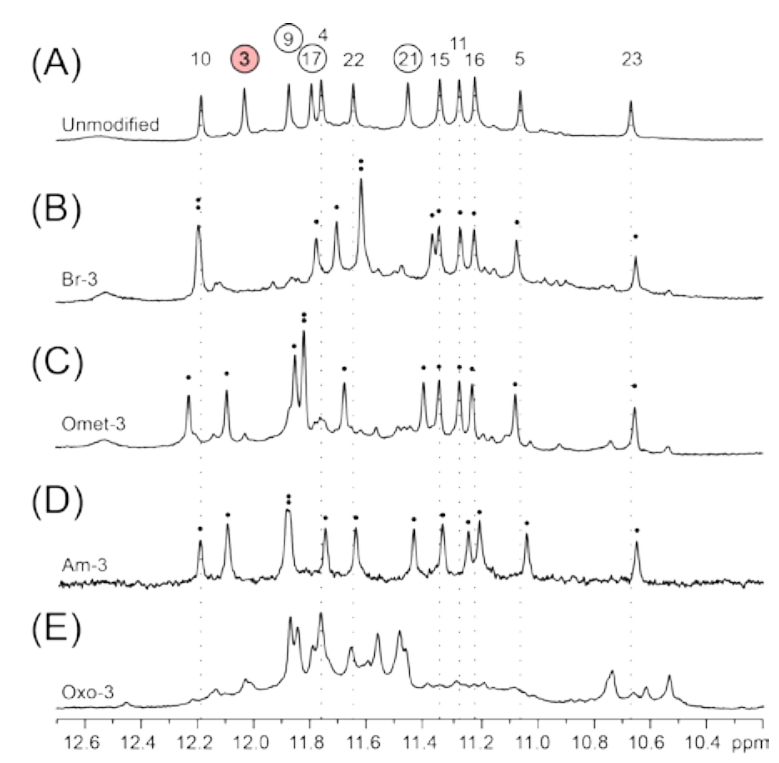

Figure 6-3: Imino proton NMR spectra for (A) the unmodified sequence, as well as modified sequences (B) Br-3, (C) Omet-3, (D) Am-3, and (E) Oxo-3 containing a single substitution to syn residue 3. The peaks in the unmodified spectrum are labeled with residue numbers, circled numbers representing guanines within the modified $\mathrm{G} 3 \cdot \mathrm{G} 21 \cdot \mathrm{G} 17 \cdot \mathrm{G} 9$ tetrad and the modified residue 3 shaded red. Dotted lines identify the chemical shift of imino protons from the unmodified spectra for guanines located outside of the modified tetrad. Black dots indicate the presence of individual imino proton resonances.

For example, in the spectra of sequences with a single ${ }^{\mathrm{Br}} \mathrm{G}$, ${ }^{\text {Omet }} \mathrm{G}$, or ${ }^{\mathrm{Am}} \mathrm{G}$ substitution at position 3 (Fig. 6-3), most changes were observed only for peaks from the modified Gtetrad $\mathrm{G} 3 \cdot \mathrm{G} 21 \cdot \mathrm{G} 17 \cdot \mathrm{G} 9$. This specific pattern in NMR chemical shift change is further investigated below. The NMR spectra of sequences containing ${ }^{\mathrm{Oxo}} \mathrm{G}$ also gave imino proton peaks at 10.5-12.5 ppm (Fig. 6-3E), indicating the formation of G-quadruplexes. However, these spectra were markedly different from that of the unmodified sequence,

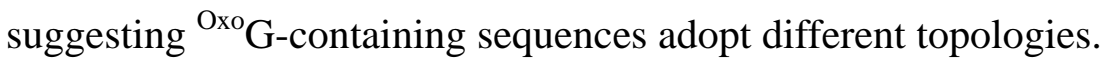

\subsection{2: Thermal stability of modified sequences}

To characterize and compare the stability of G-quadruplexes formed by different modified sequences, UV melting experiments were performed by monitoring the absorption at 295 nm (258) (Fig. 6-4). Reversible denaturing profiles were recorded for all sequences and 
Table 6-2: Melting temperatures $\left({ }^{\circ} \mathrm{C}\right)$ determined by UV absorption at $295 \mathrm{~nm}$

\begin{tabular}{|c|c|c|c|}
\hline Sequence Name & $\mathrm{T}_{\mathrm{m}}$ & $\Delta \mathrm{T}_{\mathrm{m}}$ & $\begin{array}{c}\text { Average } \\
\Delta \mathrm{T}_{\mathrm{m}}{ }^{a}\end{array}$ \\
\hline Unmodified & 57.0 & --- & --- \\
\hline $\mathrm{Br}-3$ & 59.5 & 2.5 & \\
\hline Br-9 & 63.9 & 6.9 & \\
\hline $\mathrm{Br}-15^{b}$ & 62.3 & 5.3 & $5.2 \pm 2.7$ \\
\hline Br-16 & 62.2 & 5.2 & \\
\hline $\mathrm{Br}-21^{b}$ & 63.2 & 6.2 & \\
\hline $\mathrm{Br}-22$ & 55.3 & -1.7 & --- \\
\hline Br-9-22 & 60.7 & 3.7 & --- \\
\hline Omet-3 & 59.6 & 2.6 & \\
\hline Omet-9 & 60.5 & 3.5 & \\
\hline Omet-15 & 58.9 & 1.9 & $3.1 \pm 1.4$ \\
\hline Omet-16 & 60.1 & 3.1 & \\
\hline Omet- $21^{b}$ & 61.5 & 4.5 & \\
\hline Omet-22 & 59.0 & 2.0 & --- \\
\hline Br-9-Omet-22 & 64.6 & 7.6 & --- \\
\hline Am-3 & 57.5 & 0.5 & \\
\hline Am-9 & 58.7 & 1.7 & \\
\hline Am-15 & 57.0 & 0.0 & $1.1 \pm 1.1$ \\
\hline Am-16 & 58.4 & 1.4 & \\
\hline Am-21 & 59.1 & 2.1 & \\
\hline Am-22 & 54.0 & -3.0 & --- \\
\hline Br-9-Am-22 & 59.3 & 2.3 & --- \\
\hline Oxo-3 & 47.2 & -9.8 & --- \\
\hline Oxо-9 & 38.7 & -18.3 & --- \\
\hline Oxo-15 & 38.5 & -18.5 & --- \\
\hline \multicolumn{4}{|c|}{$\begin{array}{l}\text { [a] Average } T_{m} \text { was calculated considering only syn } \\
\text { position substitutions for each modification type as } \\
\text { indicated in grey shaded regions. The “ } \pm \text { ” values indicate } \\
\text { the maximum deviations about the average. } \\
\text { [b] Samples with a small structural transition at low } \\
\text { temperature in their denaturing profile. }\end{array}$} \\
\hline
\end{tabular}

their melting temperature $\left(\mathrm{T}_{\mathrm{m}}\right)$ values are summarized in Table 6-2.

We observed that single substitutions $\left({ }^{\mathrm{Br}} \mathrm{G}\right.$, ${ }^{\text {Omet }} \mathrm{G}$ and $\left.{ }^{\mathrm{Am}} \mathrm{G}\right)$ at syn residues resulted in an increase in $\mathrm{T}_{\mathrm{m}}$ (except for the ${ }^{\mathrm{Am}} \mathrm{G}$ substitution at position 15 which showed no change), while single substitutions at anti position 22 showed $\mathrm{T}_{\mathrm{m}}$ changes that were small and varying ( $T_{m}$ decreased for ${ }^{\mathrm{Br}} \mathrm{G}$ and ${ }^{\mathrm{Am}} \mathrm{G}$ and increased for ${ }^{\text {Omet }} \mathrm{G}$ ) (Table 4-2). Among different syn guanines, the stabilization effect of base substitutions was highly positiondependent, with similar trends observed for the three types of substitutions (Fig. 6-4C). Some residues showed low stability enhancement when substituted with C8-modified guanine derivatives such as residues 3 and 15, while others showed relatively high stability enhancement such as residues 9 and 21 . When comparing the stability of sequen- 
(A)

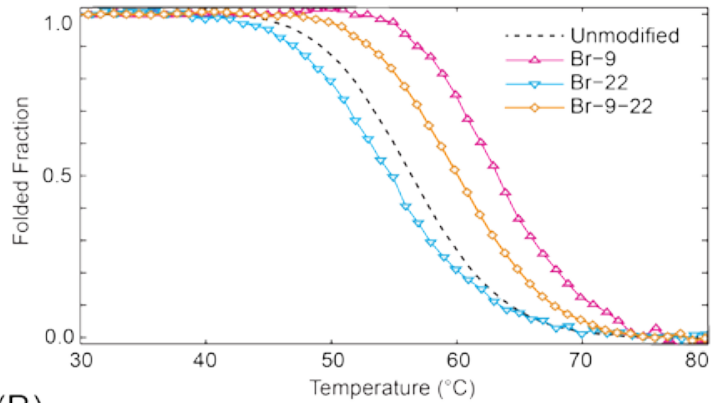

(B)

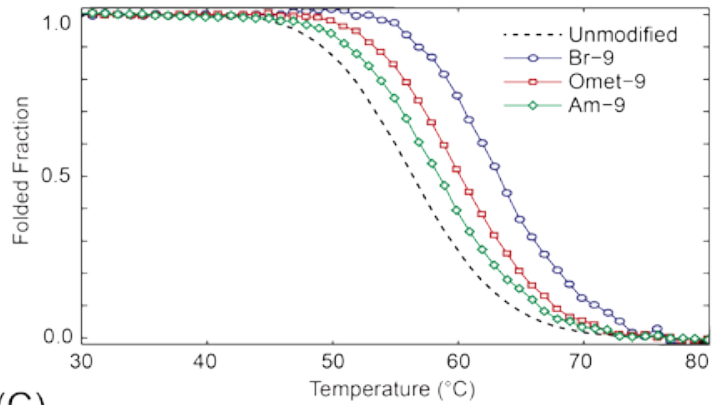

(C)

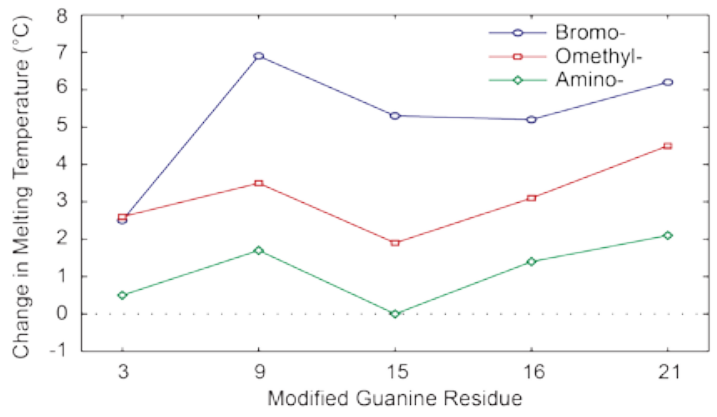

Figure 6-4: (A) The additive effect of multiple substitutions on the Tm can be seen. Melting curves for sequences Br-9 (magenta upward triangle), containing a single syn substitution; $\mathrm{Br}-22$ (cyan downward triangle), containing a single anti substitution; and Br-9-22 (orange diamonds), containing both syn and anti substitutions. The curve for the unmodified sequence (grey solid line) serves as a reference. (B) Modification dependence stability $\left({ }^{\mathrm{Br}} \mathrm{G}>{ }^{\text {Omet }} \mathrm{G}>{ }^{\mathrm{Am}} \mathrm{G}\right)$ was observed for substitutions to syn guanines, as illustrated by the melting curves of Br-9 (circles), Omet-9 (squares), and Am-9 (diamonds). The melting curve of the unmodified sequence is shown for reference. (C) Comparison of the change in $\mathrm{Tm}$ values for each guanine derivative over substitutions to various syn residues. Modifications shown as ${ }^{\mathrm{Br}} \mathrm{G}$ (blue), ${ }^{\text {Omet }} \mathrm{G}$ (red) and ${ }^{\mathrm{Am}} \mathrm{G}$ (green).

nces containing single-position syn substitutions, ${ }^{\mathrm{Br}} \mathrm{G}$ generally had the highest stabilizing effect, followed by ${ }^{\text {Omet }} \mathrm{G}$ and ${ }^{\mathrm{Am}} \mathrm{G}$, respectively (Fig. 6-4B - C). Alternatively, when substituting ${ }^{\text {Oxo }} \mathrm{G}$ to syn positions 3, 9, and 15 a large destabilizing effect was observed. Due to the large differences in NMR spectra of ${ }^{\text {Oxo }} \mathrm{G}$-containing sequences compared to that of the unmodified sequence, we do not discuss the destabilizing effects of ${ }^{\mathrm{Oxo}} \mathrm{G}$ 
modifications in terms of the $(3+1)$ G-quadruplex scaffold.

Sequences containing double substitutions showed a melting temperature reflective of the additive contributions of individual single substitution (Table 6-2). For example, the changes in $\mathrm{T}_{\mathrm{m}}$ for sequences harboring ${ }^{\mathrm{Br}} \mathrm{G}$ substitutions to only syn residue 9 , to only anti residue 22 and to both residues 9 and 22, were $+6.9,-1.7$ and $+3.7^{\circ} \mathrm{C}$, respectively (Fig. 64A and Table 6-2).

\subsection{3: A single guanine substitution in a G-tetrad results in specific patterns of NMR chemical shift changes: Experimental observations}

Analysis of the imino proton spectra of various DNA sequences containing a single ${ }^{\mathrm{Br}} \mathrm{G}$, ${ }^{\text {Omet }} \mathrm{G}$, or ${ }^{\mathrm{Am}} \mathrm{G}$ substitution at a syn guanine revealed specific patterns of chemical shift changes: (i) the most significant chemical shift changes were observed for the modified G-tetrad; (ii) the largest change was observed for the guanine that is hydrogen-bonded to the Hoogsteen edge of the modified base (note that these residues, although being neighbors in the structure, are usually remote from each other in the sequence); (iii) the magnitude and direction of chemical shift changes depended on the type of modification.

Consider a $G_{\alpha} \bullet G_{\beta} \bullet G_{\gamma} \bullet G_{\delta}$ tetrad, in which $G_{\alpha}$ is the modified base (Fig. 6-5A), the largest change in imino proton chemical shift would be observed for $\mathrm{G}_{\delta}$, which is hydrogenbonded to the Hoogsteen edge of $G_{\alpha}$. For each sequence with a single ${ }^{\mathrm{Br}} \mathrm{G}$, ${ }^{\text {Omet }} \mathrm{G}$ or ${ }^{\mathrm{Am}} \mathrm{G}$ substitution at a syn guanine, the imino proton of $G_{\delta}$ was unambiguously assigned using site-specific ${ }^{15} \mathrm{~N}$-labeling approach (259) (e.g. Fig. 6-5F). Table 6-3 lists the changes in chemical shifts for sequences containing single substitutions at syn guanines. For ${ }^{\mathrm{Br}} \mathrm{G}$ substitutions, the $G_{\delta}$ imino proton chemical shift decreased by $0.26-0.39$ ppm (Fig. 6-5C 
(A)
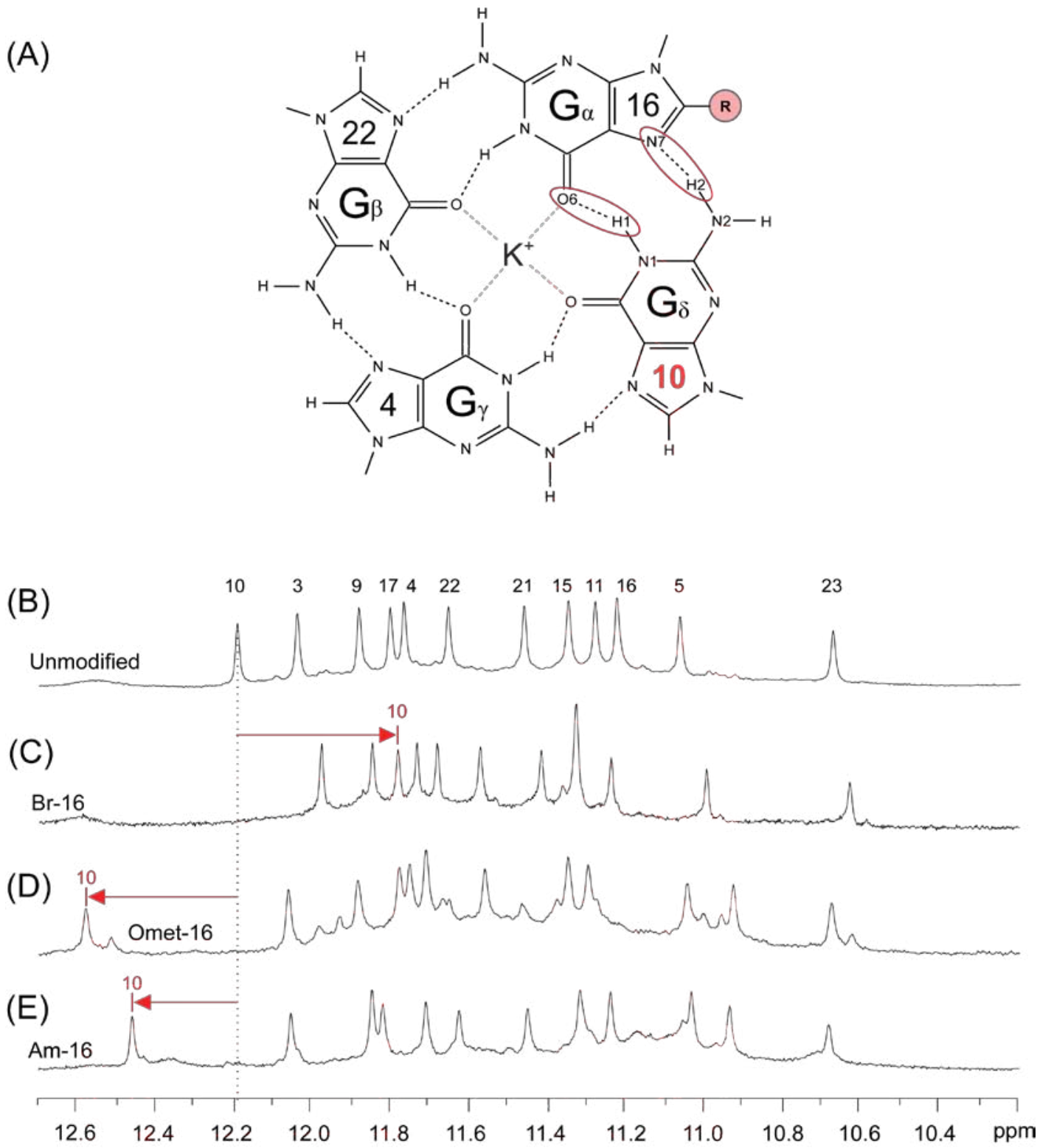

$(\mathrm{F})$

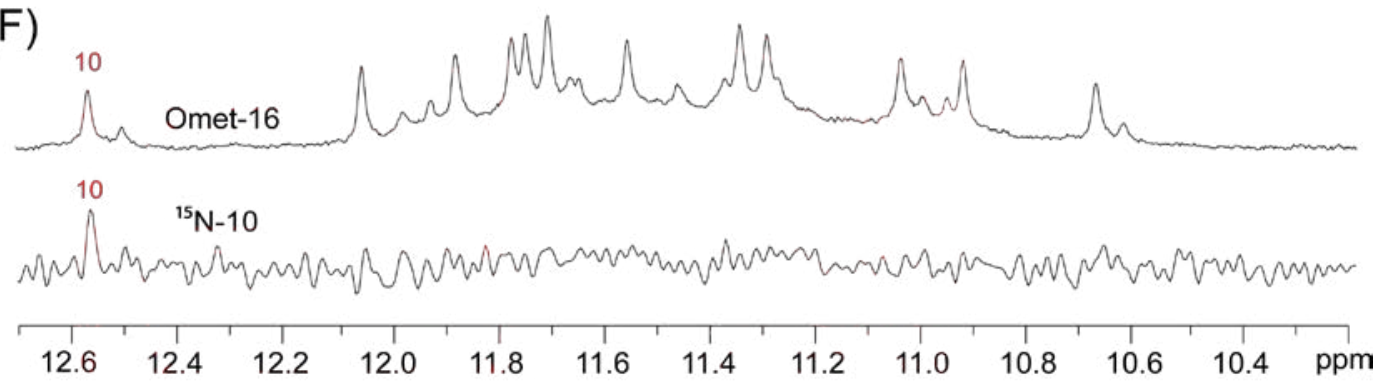

Figure 6-5: (A) Schematic of the modified G16 $\bullet$ G22 $\bullet \mathrm{G} 4 \cdot \mathrm{G} 10$ tetrad is presented with the $\mathrm{G}_{\alpha} \bullet \mathrm{G}_{\beta} \bullet \mathrm{G}_{\gamma} \bullet \mathrm{G}_{\delta}$ naming convention. Special attention is brought to the hydrogen bonds of the $G_{\delta} \bullet G_{\alpha}$ interface. (B-E) The NMR imino proton spectrum of $(\mathbf{B})$ the unmodified sequence is presented along with the spectra of $(\mathbf{C}) \mathrm{Br}$ 16, (D) Omet-16, and (E) Am-16. The chemical shift of the imino proton peak from the $\mathrm{G}_{\delta}$ residue, G10, in the unmodified sequence is indicated by the dotted black line. Arrows indicate the positions of the $\mathrm{G}_{\delta}$ imino proton peaks in the modified spectra. (F) Example of the unambiguous G10 imino proton assignment for Omet-16 using ${ }^{15} \mathrm{~N}$-labeling. 
Table 6-3. Experimentally observed changes in NMR H1 chemical shift of the $\mathrm{G}_{\delta}$ imino proton

\begin{tabular}{|c|c|c|c|c|c|}
\hline \multirow{3}{*}{$\begin{array}{l}\text { Modification } \\
\text { Type }\end{array}$} & \multicolumn{5}{|c|}{ Change in $\mathrm{G}_{\delta} \mathrm{H} 1$ Chemical Shift (ppm) } \\
\hline & \multicolumn{5}{|c|}{ Neighboring residue $\left(\mathrm{G}_{\delta}\right) \bullet$ Modified Residue $\left(\mathrm{G}_{\alpha}\right)$} \\
\hline & $9 \cdot 3$ & $17 \cdot 9$ & $11 \cdot 15$ & $10 \cdot 16$ & $3 \cdot 21$ \\
\hline 8-BromoG & -0.26 & -0.32 & -0.28 & -0.39 & -0.28 \\
\hline 8-O-methylG & 0.22 & 0.24 & 0.40 & 0.39 & 0.30 \\
\hline 8-AminoG & 0.21 & 0.26 & 0.26 & 0.27 & 0.19 \\
\hline
\end{tabular}

and Table 6-3). Conversely, for ${ }^{\text {Omet }} \mathrm{G}$ and ${ }^{\mathrm{Am}} \mathrm{G}$ substitutions, the $\mathrm{G}_{\delta}$ imino proton peak was downfield-shifted; the chemical shift increased by $0.22-0.40$ and $0.19-0.27 \mathrm{ppm}$, respectively (Fig. 6-5D-E and Table 6-3).

Tentative spectral assignments also suggested a specific pattern of chemical shift changes for the imino proton of the modified guanine $G_{\alpha}$, which was in the opposite direction of the $\mathrm{G}_{\delta}$ imino proton and in agreement with computational studies. However, as these assignments were not unambiguously confirmed, we will not discuss their patterns here.

\subsection{4: A single guanine substitution in a G-tetrad results in specific patterns of NMR chemical shift changes: Computational studies}

In order to better understand the chemical shift patterns described above, we attempted to reproduce the experimentally observed patterns of chemical shift change due to base modification using a series of $a b$ initio computational studies. Computations were performed at the Hartree-Fock level (260) using a variety of basis sets as described in Appendix II - Protocols - Chapter 6. NMR chemical shift calculations were performed on optimized molecular geometries for modified and unmodified tetrads. The changes in NMR chemical shifts between the unmodified and modified systems are shown in Table 6-4. All calculation protocols employed were able to reproduce the experimentally observed trends in $\mathrm{G}_{\delta}$ imino proton chemical shift changes, with a decrease of 0.21-0.31 
Table 6-4: Computed change in imino proton (H1) NMR chemical shift of the $\mathrm{G}_{\delta}$ residue

\begin{tabular}{|c|c|c|c|c|c|c|}
\hline \multirow{2}{*}{$\begin{array}{l}\text { Modification } \\
\text { Type }\end{array}$} & \multirow{2}{*}{$\begin{array}{l}\text { Optimization } \\
\text { Basis Set }\end{array}$} & \multicolumn{5}{|c|}{$\begin{array}{c}\text { Computed Change in } \mathrm{G}_{\delta} \mathrm{H} 1 \text { Chemical Shift (ppm) } \\
\text { NMR Basis Set }\end{array}$} \\
\hline & & 6-31G(d) & 6-31G(d,p) & $\begin{array}{c}6- \\
31++G(d, p)\end{array}$ & $6-311+G(d)$ & $6-311++G(d, p)$ \\
\hline \multirow[t]{4}{*}{ 8-BromoG } & 6-31G(d) & -0.289 & -0.311 & -0.304 & -0.300 & -0.307 \\
\hline & 6-31G(d,p) & -0.289 & -0.311 & -0.305 & -0.300 & -0.307 \\
\hline & 6-31++G(d,p) & -0.235 & -0.249 & -0.243 & -0.245 & -0.242 \\
\hline & $6-311++G(d, p)$ & -0.208 & -0.216 & -0.208 & -0.214 & -0.205 \\
\hline \multirow[t]{4}{*}{ 8-O-methylG } & $6-31 G(d)$ & 0.398 & 0.461 & 0.442 & 0.347 & 0.453 \\
\hline & 6-31G(d,p) & 0.402 & 0.465 & 0.447 & 0.351 & 0.458 \\
\hline & 6-31++G(d,p) & 0.398 & 0.461 & 0.443 & 0.350 & 0.455 \\
\hline & $6-311++G(d, p)$ & 0.424 & 0.489 & 0.472 & 0.376 & 0.484 \\
\hline \multirow[t]{4}{*}{ 8-AminoG } & $6-31 G(d)$ & 0.235 & 0.272 & 0.261 & 0.234 & 0.279 \\
\hline & 6-31G(d,p) & 0.241 & 0.279 & 0.267 & 0.239 & 0.285 \\
\hline & 6-31++G(d,p) & 0.242 & 0.280 & 0.269 & 0.242 & 0.288 \\
\hline & $6-311++G(d, p)$ & 0.248 & 0.286 & 0.275 & 0.247 & 0.294 \\
\hline
\end{tabular}

ppm calculated for the ${ }^{\mathrm{Br}} \mathrm{G}$-containing model and an increase of 0.35-0.49 ppm and 0.23-

0.29 ppm calculated for the ${ }^{\text {Omet }} \mathrm{G}$ - and ${ }^{\text {Am }}$ G-containing models, respectively (Fig. 6-6).

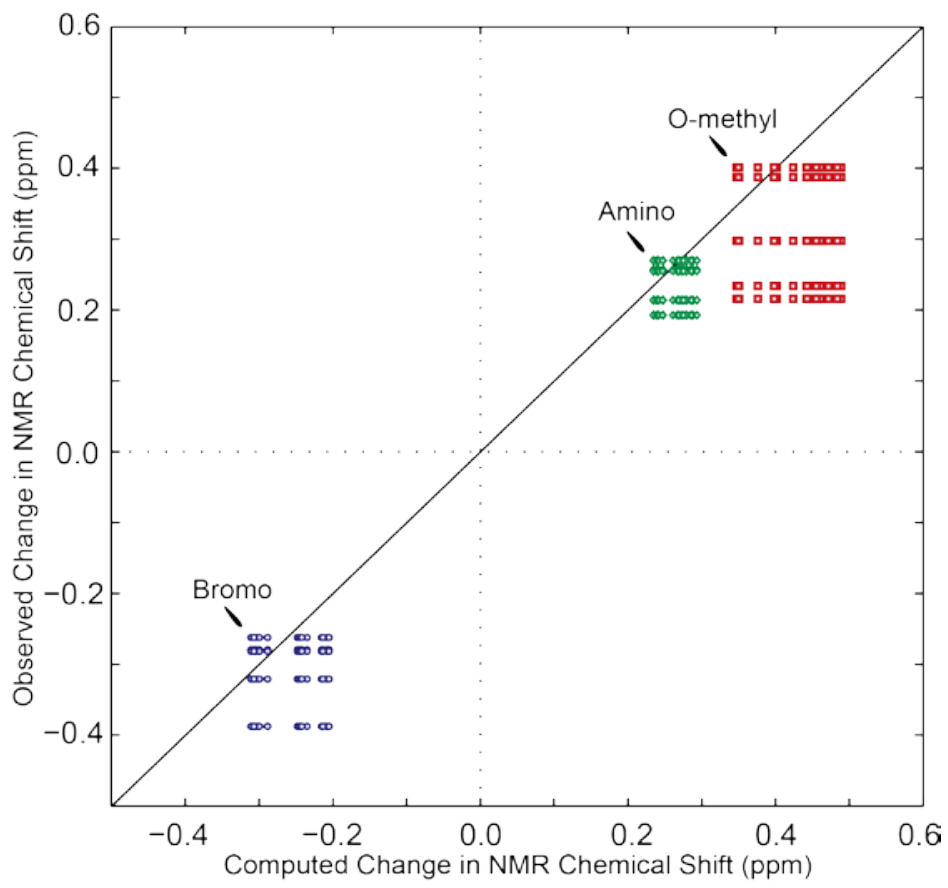

Figure 6-6: Comparison of experimental and computed NMR chemical shift changes. Experimental data are observed chemical-shift changes of the $\mathrm{G}_{\delta}$ imino protons upon substitutions to syn residues $3,9,15,16$, and 21 by guanine derivatives ${ }^{\mathrm{Br}} \mathrm{G}$, ${ }^{\text {Omet }} \mathrm{G}$, and ${ }^{\mathrm{Am}} \mathrm{G}$. Computational data are the results of different NMR chemical-shift calculations, as shown in Table 6-4. The solid black line represents exact agreement between computational and experimental results. Data points are shown for every combination of experimentally observed and computed changes in chemical shift for each type of guanine derivative. 


\subsection{5: Structural effects of C8-modifications on G-tetrad geometry: An ab initio computational study}

As our computational studies correctly reproduced the experimentally observed chemical shift patterns, we used these computational methods to analyze the structural effects of C8-base modifications on G-tetrad geometry. It is generally revealed that the most significant changes in geometry occurred at the $G_{\delta}{ }^{\bullet} G_{\alpha}$ and $G_{\alpha} \cdot G_{\beta}$ interfaces (refer to Fig. 6-5A). Calculated changes to imino and amino hydrogen-bond geometries at the $G_{\delta} \bullet G_{\alpha}$ interface are presented in Table 6-5 and Table 6-6, respectively. Comparison between the changes in $\mathrm{G}_{\delta}$ imino proton chemical shifts and the changes in $\mathrm{H}_{1 \delta} \bullet \bullet \mathrm{O}_{6 \alpha}$ bond length relates an increase in imino proton chemical shifts with a decrease in hydrogen-bond lengths, and vice versa. In addition, partial atomic charges were derived from the electrostatic potentials of tetrads with optimized geometries. We were unable to observe a relationship between partial atomic charges and NMR chemical shift changes, as the calculated partial charge highly depended on the basis set used for geometry optimization.

Table 6-5. Computed effects of guanine substitutions on N1-H•••O6 geometry of the tetrad

\begin{tabular}{|c|c|c|c|c|c|c|c|c|c|}
\hline \multirow{2}{*}{$\begin{array}{c}\text { Modification } \\
\text { Type }\end{array}$} & \multirow{2}{*}{$\begin{array}{c}\text { Optimization } \\
\text { Basis Set }\end{array}$} & \multicolumn{4}{|c|}{$\Delta \mathrm{H} 1 \cdots \mathrm{O} 6(\mathrm{pm})$} & \multicolumn{4}{|c|}{$\Delta$ N1 - H1 $\cdots$ O6 Angle (deg) } \\
\hline & & $\mathrm{G}_{\alpha} \cdot \mathrm{G}_{\beta}$ & $\mathrm{G}_{\beta} \cdot \mathrm{G}_{\gamma}$ & $\mathrm{G}_{\gamma} \cdot \mathrm{G}_{\delta}$ & $\mathrm{G}_{\delta} \bullet \mathrm{G}_{\alpha}$ & $\mathrm{G}_{\alpha} \cdot \mathrm{G}_{\beta}$ & $\mathrm{G}_{\beta} \cdot \mathrm{G}_{\gamma}$ & $\mathrm{G}_{\gamma} \cdot \mathrm{G}_{\delta}$ & $\mathrm{G}_{\delta} \cdot \mathrm{G}_{\alpha}$ \\
\hline \multirow[t]{4}{*}{ 8-BromoG } & $6-31 G(d)$ & -0.97 & 0.99 & 1.57 & 5.02 & -1.12 & -0.12 & 0.00 & -1.51 \\
\hline & 6-31G(d,p) & -0.96 & 0.97 & 1.52 & 4.92 & -1.07 & -0.11 & 0.00 & -1.45 \\
\hline & 6-31++G(d,p) & -1.05 & 0.44 & 0.66 & 2.96 & -0.60 & 0.00 & -0.01 & -0.69 \\
\hline & 6-311++G(d,p) & -0.50 & 0.18 & 0.63 & 1.80 & -0.11 & 0.08 & -0.08 & -0.18 \\
\hline \multirow[t]{4}{*}{ 8-O-methylG } & 6-31G(d) & 1.08 & -1.97 & -2.11 & -10.14 & 3.80 & 0.46 & -0.22 & 3.35 \\
\hline & 6-31G(d,p) & 1.00 & -1.98 & -2.11 & -10.10 & 3.77 & 0.45 & -0.22 & 3.31 \\
\hline & 6-31++G(d,p) & 1.13 & -2.10 & -2.11 & -10.26 & 3.89 & 0.47 & -0.21 & 3.41 \\
\hline & 6-311++G(d,p) & 0.92 & -2.44 & -2.23 & -11.02 & 4.07 & 0.56 & -0.18 & 3.60 \\
\hline \multirow[t]{4}{*}{ 8-AminoG } & 6-31G(d) & -0.15 & -1.06 & -2.49 & -6.25 & 2.15 & 0.21 & 0.19 & 1.60 \\
\hline & 6-31G(d,p) & -0.14 & -1.08 & -2.50 & -6.30 & 2.12 & 0.21 & 0.18 & 1.60 \\
\hline & 6-31++G(d,p) & -0.08 & -1.18 & -2.51 & -6.51 & 2.21 & 0.23 & 0.18 & 1.71 \\
\hline & $6-311++G(d, p)$ & -0.35 & -1.36 & -2.70 & -6.83 & 2.30 & 0.29 & 0.24 & 1.79 \\
\hline
\end{tabular}

Geometry changes listed reflect variation from the original values of bond length and angle as found in the unmodified optimized structures. Unmodified tetrad hydrogen bond geometries obtained are as follows: 6-31G(d): $214.33 \mathrm{pm}$ and $157.04^{\circ}, 6-31 G(d, p)$ : $214.07 \mathrm{pm}$ and $157.18^{\circ}, 6-31++G(d, p): 215.08 \mathrm{pm}$ and $157.76^{\circ}, 6-311++G(d, p): 216.27 \mathrm{pm}$ and $157.14^{\circ}$. Listed angles were measured toward the center of the tetrad. 


\section{Chapter 6: C8-modified Guanines}

Table 6-6. Computed effects of guanine substitutions on N2-H•••N7 geometry of the tetrad

\begin{tabular}{|c|c|c|c|c|c|c|c|c|c|}
\hline \multirow{2}{*}{$\begin{array}{c}\text { Modification } \\
\text { Type }\end{array}$} & \multirow{2}{*}{$\begin{array}{l}\text { Optimization } \\
\text { Basis Set }\end{array}$} & \multicolumn{4}{|c|}{$\Delta \mathrm{H} 2 \bullet \mathrm{N} 7(\mathrm{pm})$} & \multicolumn{4}{|c|}{$\Delta \mathrm{N} 2-\mathrm{H} 2 \cdot \bullet$ N7 Angle (deg) } \\
\hline & & $\mathrm{G}_{\alpha} \cdot \mathrm{G}_{\beta}$ & $\mathrm{G}_{\beta} \cdot \mathrm{G}_{\gamma}$ & $\mathrm{G}_{\gamma} \cdot \mathrm{G}_{\delta}$ & $\mathrm{G}_{\delta} \cdot \mathrm{G}_{\alpha}$ & $\mathrm{G}_{\alpha} \cdot \mathrm{G}_{\beta}$ & $\mathrm{G}_{\beta} \bullet \mathrm{G}_{\gamma}$ & $\mathrm{G}_{\gamma} \cdot \mathrm{G}_{\delta}$ & $\mathrm{G}_{\delta} \cdot \mathrm{G}_{\alpha}$ \\
\hline \multirow[t]{3}{*}{ 8-BromoG } & $6-31 G(d)$ & 0.46 & 0.41 & 0.02 & 1.95 & -0.90 & -0.13 & -0.06 & -2.58 \\
\hline & $6-31++G(d, p)$ & -0.44 & 0.09 & -0.07 & 1.92 & -0.54 & -0.02 & -0.03 & -1.23 \\
\hline & $6-311++G(d, p)$ & -0.58 & -0.17 & -0.13 & 2.76 & -0.17 & 0.05 & -0.08 & -0.49 \\
\hline \multirow[t]{3}{*}{ 8-O-methylG } & $6-31 G(d)$ & -2.77 & -1.64 & -0.37 & 2.50 & 2.31 & 0.42 & -0.11 & 4.17 \\
\hline & $6-31++G(d, p)$ & -3.01 & -1.75 & -0.45 & 3.01 & 2.40 & 0.44 & -0.11 & 4.24 \\
\hline & $6-311++G(d, p)$ & -3.25 & -1.89 & -0.52 & 2.97 & 2.52 & 0.52 & -0.08 & 4.33 \\
\hline \multirow[t]{3}{*}{ 8-AminoG } & $6-31 G(d)$ & -0.84 & -0.76 & -0.28 & -2.78 & 1.02 & 0.20 & 0.22 & 2.53 \\
\hline & 6-31G(d,p) & -0.86 & -0.77 & -0.27 & -2.91 & 1.00 & 0.19 & 0.21 & 2.53 \\
\hline & 6-31++G(d,p) & -0.98 & -0.82 & -0.30 & -2.56 & 1.07 & 0.21 & 0.21 & 2.65 \\
\hline
\end{tabular}

Geometry changes listed reflect variation from the original values of bond length and angle as found in the unmodified optimized structures. Unmodified tetrad hydrogen bond geometries obtained are as follows: $6-31 G(d)$ : 214.33 pm and $157.04^{\circ}, 6-31 G(d, p)$ : $214.07 \mathrm{pm}$ and $157.18^{\circ}, 6-31++G(d, p)$ : $215.08 \mathrm{pm}$ and $157.76^{\circ}, 6-311++G(d, p)$ : $216.27 \mathrm{pm}$ and $157.14^{\circ}$. Listed angles were measured toward the center of the tetrad. 


\subsection{Discussion:}

This study examines the effects of site-specific base modifications of four C8-substituted guanine derivatives $\left({ }^{\mathrm{Br}} \mathrm{G}\right.$, ${ }^{\text {Omet }} \mathrm{G},{ }^{\mathrm{Am}} \mathrm{G}$, and $\left.{ }^{\text {Oxo }} \mathrm{G}\right)$ into various positions of a G-quadruplex structure. The chosen DNA sequence formes an intramolecular (3+1) G-quadruplex with well-defined spectroscopic characteristics, and the folding topology of the G-quadruplex was controlled and monitored by CD and NMR spectra, allowing a quantitative comparison of the effects of base modifications in different positions of the same Gquadruplex fold for ${ }^{\mathrm{Br}} \mathrm{G}$, ${ }^{\text {Omet }} \mathrm{G}$ and ${ }^{\mathrm{Am}} \mathrm{G}$ modifications. Although minor conformations were present for some modified sequences, their relatively small populations should not affect the interpretations regarding the stability of the major fold.

This work reports that single-position ${ }^{\mathrm{Br}} \mathrm{G}$, ${ }^{\text {Omet }} \mathrm{G}$, and ${ }^{\mathrm{Am}} \mathrm{G}$ substitutions to the five syn guanines of a $(3+1)$ G-quadruplex scaffold generally lead to an increase in thermal stability. These results agree with past findings that substitutions of syn guanines by C8modified guanine derivatives 8-bromo-guanines $(25,113)$ and 8-methyl-guanines (118) stabilize G-quadruplexes and extend this conclusion for 8-O-methyl-guanine and 8amino-guanine substitutions. Among the C8-modifications explored, ${ }^{\mathrm{Br}} \mathrm{G}$ was found to be generally most stabilizing followed by ${ }^{\text {Omet }} \mathrm{G}$ and ${ }^{\mathrm{Am}} \mathrm{G}$ respectively (Fig. 6-4B and Table 6-2). Thermal denaturing results indicate a position-dependent stabilization effect of single C8-modified derivatives among the various syn residues (Fig. 6-4C). This positiondependency may be explained by the different local structural environments among the various syn positions, such as neighboring loops, grooves, and tetrad layers. A positiondependence effect has also been demonstrated in other scaffolds containing C8modifications $(113,118)$. 
While the expected stabilization of G-quadruplexes by C8-modified guanine substitutions ${ }^{\mathrm{Br}} \mathrm{G}$, ${ }^{\text {Omet }} \mathrm{G}$ and ${ }^{\mathrm{Am}} \mathrm{G}$ to syn positions was confirmed, it was unanticipated to observe the stabilization effect of an ${ }^{\text {Omet }} \mathrm{G}$ substitution to anti guanine $\mathrm{G} 22$ as previous studies exploring C8-modified guanine substitutions to anti positions in intramolecular scaffolds have reported destabilizing effects $(25,113,261)$. To our knowledge, this is the first reported observation of a site-specific C8-modified substitution (8-O-methyl-guanine) stabilizing an intramolecular G-quadruplex system when substituted into an anti position. Similarities between the CD and imino proton NMR spectra of the sequence containing an ${ }^{\text {Omet }} \mathrm{G}$ substitution at position 22 and those of the unmodified sequence suggests that the same folding topology is maintained and the 8-O-methyl-guanine adopts an anti conformation in this modified sequence. It is speculated that ${ }^{\text {Omet }} \mathrm{G}$ may constitute a unique type of modification due to its ability to rotate about the $\mathrm{N}_{7}-\mathrm{C}_{8}-\mathrm{O}_{\mathrm{R}}-\mathrm{C}_{\mathrm{R}}$ bond. The ability for anti positions to tolerate the inclusion of C8-modified guanine derivatives may be dependent on specific structural features. However, the generalization of such dependence would require more systematic study and is outside the scope of this work. Substitutions were also made to anti residue 23 with destabilizing effects observed for ${ }^{\mathrm{Br}} \mathrm{G},{ }^{\mathrm{Am}} \mathrm{G}$, and ${ }^{\text {Omet }} \mathrm{G}$. The effects of modifications to residue 23 are not discussed here as the folding topology could not be confirmed due to significant changes in CD and NMR spectra.

The observation of specific NMR chemical shift patterns due to base modifications could potentially be a tool in structural elucidation or verification, as the observed crosshydrogen-bond effect occurs at a remote site in the sequence. For example, in the $(3+1)$ human telomeric G-quadruplex structure considered above, a modification at position 3 would cause a predictable change in imino proton chemical shift of residue 9, while a modification at position 9 would cause a change in position 17, and so forth. In this 
manner, site-specific modifications could be used in NMR studies to identify Hoogsteen hydrogen-bound guanines within a G-quadruplex structure.

Quantum mechanical calculations of NMR chemical shifts provide a valuable link between experimentally observable chemical shifts and calculated molecular geometries $(262,263)$. Previously, NMR was used to probe the effects of temperature and ion type on hydrogen-bond lengths within G-tetrads (264). The changes in NMR observables, namely N7 chemical shifts and J-couplings, were interpreted in terms of hydrogen-bond length and substantiated by quantum mechanical calculations. A similar approach was also used to study hydrogen-bond length and strength in a triple helix (263).

In this manner, observed chemical shift patterns were used to investigate the effects of modifications on hydrogen-bond geometry. In this study, $a b$ initio NMR chemical shift calculations were found to reproduce the observed changes in $G_{\delta}$ imino proton chemical shifts to a high degree (Fig. 6-6, Table 6-3 and Table 6-4). The difference between modification-driven chemical shift changes calculated by different methods was within a range of $0.11 \mathrm{ppm}, 0.14 \mathrm{ppm}$ and $0.06 \mathrm{ppm}$ for the ${ }^{\mathrm{Br}} \mathrm{G}-$, ${ }^{\text {Omet }} \mathrm{G}$ - and ${ }^{A m} \mathrm{G}$-containing models, respectively. These data are in agreement with studies by Kumar and McAllister (262), indicating that relative NMR chemical shift calculations are quite accurate.

The computed effects of modifications on tetrad geometries revealed the largest change in hydrogen-bond geometry at the $\mathrm{G}_{\delta} \bullet \mathrm{G}_{\alpha}$ interface (Table 6-5 and Table 6-6). A relationship between decreasing hydrogen-bond length and downfield-shifting (increased ppm) of imino proton chemical shifts, and vice-versa, was observed. This trend was obtained across all choices of geometry optimization basis sets explored in this study. These results are in agreement with previous studies $(262,263)$ which reported a similar relationship 
between hydrogen-bond length and proton chemical shift. It is important to note that NMR chemical shift calculations performed on modified tetrads prior to geometry reoptimization (data not shown) did not fully reproduce experimental chemical shifts, especially for the ${ }^{\text {Omet }} \mathrm{G}$ - and ${ }^{\mathrm{Am}} \mathrm{G}$-containing models. This indicates that a structural change in G-tetrad geometry is an important feature of modification-driven changes in NMR chemical shift.

Modification-driven change in hydrogen-bond geometry may not be the only factor contributing to the observed changes in imino proton chemical shift. Changes in base stacking geometry caused by C8-substitutions may lead to changes in the ring-current driven magnetic shielding experienced by imino protons. However, a similar pattern in NMR chemical shift change was experimentally observed for guanines from all three Gtetrad layers in the structure. This indicates that for the modifications explored here, the effect of base stacking changes may be small.

Understanding the effects of C8-modifications on stability, as well as the hydrogen-bond geometry of a G-tetrads, should be valuable in the engineering of new G-quadruplexes, as well as the design of molecules targeting these structures. Computational models explored here, as supported by the agreement between computational and experimental NMR data, show great potential in investigation of the effects of different guanine derivatives on tetrad structure. 


\subsection{Conclusion:}

We examined the effects of site-specific substitution of guanines with the guanine derivatives 8-bromo-guanine, 8-O-methyl-guanine, 8-amino-guanine, and 8-oxo-guanine, using a well-defined (3+1) G-quadruplex as the platform. It was observed that 8-bromoguanine has the highest stabilizing effect when substituted with a single syn guanine, followed by 8-O-methyl-guanine and 8-amino-guanine. Substitutions of 8-oxo-guanine lead to drastic changes in NMR imino proton spectra and a large decrease in stability. The effect of substitution on the stability of the G-quadruplex is highly position-dependant among different guanines in the structure. The C8-modification of a guanine results in a specific pattern of chemical shift changes: the imino proton of the guanine that is hydrogen-bonded to the modified base experiences the largest chemical shift change, which depends on the type of modification. This specific chemical shift pattern was successfully reproduced by $a b$ inito quantum mechanical computations. The computational study performed here suggests the effects of various C8-modified guanine substitutions on the Hoogsteen hydrogen-bond geometry within a G-tetrad. 


\section{Chapter 7:}

\section{Engineering the Base: N2-modified Guanines}

This chapter was largely adapted from the authors work: Lech, C.J. and Phan, A.T. (2013) Ball with Hair: functionalization of extremely stable G-quadruplex nanoparticles through N2-guanine modifications. (Submitted)

Author Contributions: DNA synthesis, purification and biophysical experiments were carried out by C.J.L.; Data analysis of thermal denaturing experiments was performed by C.J.L.; Both Authors wrote the above mentioned manuscript and contributed to the conceptualization and planning of experiments.

\subsection{Introduction:}

G-quadruplexes are useful molecular scaffolds, self-assembling and self-folding in an iondependent manner into thermally stable nanoparticles (57). A major advantage of nucleic acid-based nanoparticles is their ability to be synthesized and functionalized in a controlled and spatial-specific manner. For example, functionalization of strand termini can enhance the activity of G-quadruplex based aptamers (265) and enables their use as FRET based sensors (266). While modification of strand termini is useful, such modifications are restricted by the location and number of strand termini in the Gquadruplex molecule. Modifications can also be made to the loops of G-quadruplexes, but even slight changes in loop base chemistry can often alter the G-quadruplex conformations adopted $(23,233)$. Another strategy for modification of G-quadruplex is to substitute chemically-altered guanines bases into the G-tetrad core of the G-quadruplex (107). In principle, modification of the guanine base allows for functionalization at a 
desired location on the G-quadruplex nanoparticle, and can accommodate multiple modified guanines per tetrad layer. Additionally, modification of the base can be done in parallel to modification to other regions of the G-quadruplex, allowing for numerous possible routes to nanoparticle functionalization.

However, many types of guanine base analogs have been observed to destabilize the Gquadruplex structure $(111,112)$. Modification at the C8 position of the guanine base has received a fair amount of research attention (107). As discussed in Chapter 6, C8modifications affect the stability of G-quadruplex formation in a manner dependent on the glycosidic conformation of the guanine base $(25,113)$. This conformational dependence limits the ability to functionalize at the C8-position, with only substitutions to guanines in a syn-conformation being effectively incorporated.

By comparison, the N2-position of the guanine base has received far less research attention (122,123,267-269). In principle, chemical substitution of a single amino proton at the N2-position does not disrupt the Hoogsteen bonding of the G-tetrad. With this in mind, we set out to characterize the effects of a variety of N2-modified guanines bases on the formation and stability of G-quadruplex nanoparticles. Surprisingly, our results reveal that N2-modified guanines are potent stabilizers of the G-quadruplex architecture and can be substituted in a site-specific manner into a variety of locations on the G-quadruplex nanoparticle scaffold without disrupting nanoparticle formation. Furthermore, multiple N2-modifications may be incorporated simultaneously leading to highly stabilized Gquadruplex nanoparticles. We present the design of a "Ball-with-Hair" nanoparticle containing multiple “chain-like” N2-6-amino-hexyl-guanine chemistries serving as potential sites for nanoparticle functionalization. 


\subsection{Results and Discussion:}

\subsection{1: G-quadruplex nanoparticle formation of sequences containing single N2-guanine modifications}

In our efforts to functionalize a G-quadruplex scaffold with N2-modified guanine bases, we systematically substituted N2-methyl-guanine ( $\left.{ }^{\mathrm{Met}} \mathrm{G}\right)$, N2-6-amino-hexyl-guanine $\left({ }^{\text {Hex }} \mathrm{G}\right)$, and N2-benzyl-guanine $\left({ }^{\text {Ben }} \mathrm{G}\right)$ into the G-quadruplex structure formed from the sequence $d\left[T_{2}\left(G_{3} T_{2} A\right)_{3} G_{3} A\right]$ (Fig 7-1). This sequence has been previously shown to form a well-defined $(3+1)$ G-quadruplex conformation with three strands oriented in one direction and one pointing in the opposite direction (28). Connecting the strands of the Gquadruplex are a fold-back propeller loop and two edge-wise loops. The orientation of the strands give rise to grooves of varying widths, into which the N2-modification of the guanine base would be projected (Fig 7-1B). Groove widths are classified as wide $(W)$, medium $(M)$, or narrow $(N)$ based upon the geometry of the backbones which define the groove. We use an additional groove description to characterize the medium groove containing a fold-back propeller loop $(P)$. The guanine-tetrad (G-tetrad) core of the molecule is characterized by 3 stacked guanine tetrads with guanine bases in both syn and
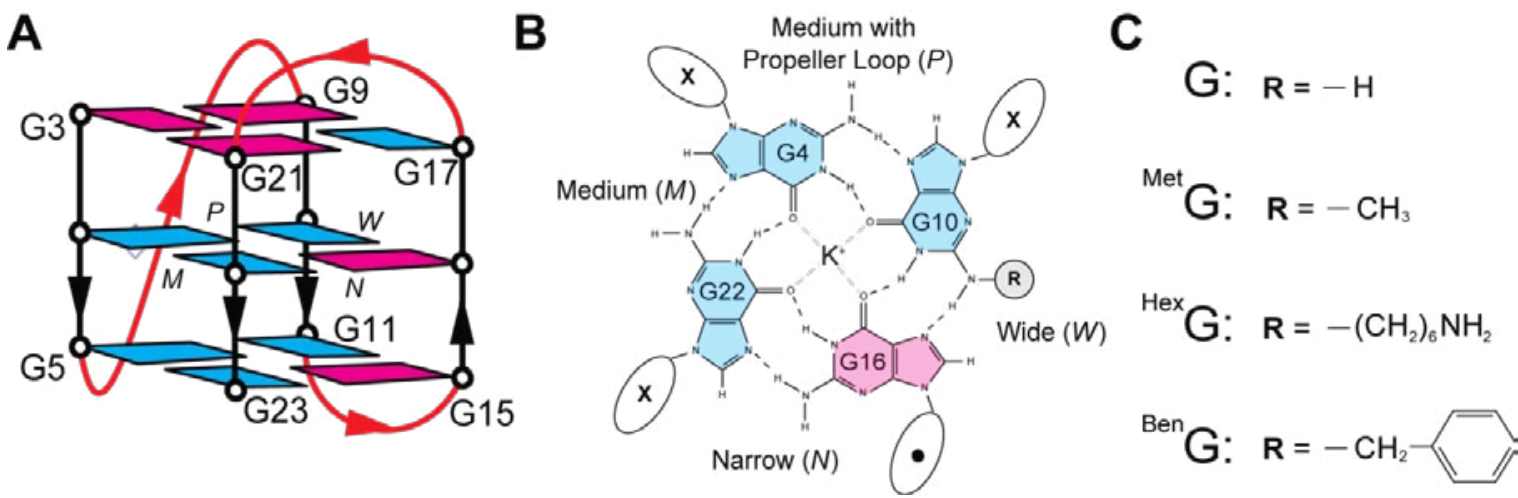

Figure 7-1: Comparison of experimental and computed (A) Schematic of the $(3+1)$ hybrid G-quadruplex studied in this work. Groove widths and select guanine residues are labeled. Guanine bases are colored based on their syn (magenta) or anti (cyan) glycosidic conformation. (B) Example of a single-position N2modified guanine substitution within the middle G-tetrad layer. Groove descriptions are shown. The 5' $\rightarrow 3$ ' strand directionality is indicated as into (x) and out of $(\bullet)$ the page. (C) Description of the N2-guanine modifications explored in this work. 
Table 7-1: Sequences studied in this work

\begin{tabular}{|c|c|c|c|c|c|c|c|c|c|}
\hline Name & Seq & ence & & & & & & & \\
\hline Unmodified & $\mathrm{TT}$ & GGG & TTA & GGG & TTA & GGG & TTA & GGG & A \\
\hline Met-3 & $\mathrm{TT}$ & MGG & TTA & GGG & TTA & GGG & TTA & GGG & A \\
\hline Met-4 & $\mathrm{TT}$ & GMG & TTA & GGG & TTA & GGG & TTA & GGG & A \\
\hline Met-5 & $\mathrm{TT}$ & GGM & TTA & GGG & TTA & GGG & TTA & GGG & A \\
\hline Met -9 & $\mathrm{TT}$ & GGG & TTA & MGG & TTA & GGG & TTA & GGG & A \\
\hline Met -10 & $\mathrm{TT}$ & GGG & TTA & GMG & TTA & GGG & TTA & GGG & A \\
\hline Met -11 & $\mathrm{TT}$ & GGG & TTA & GGM & TTA & GGG & TTA & GGG & A \\
\hline Met -15 & $\mathrm{TT}$ & GGG & TTA & GGG & TTA & MGG & TTA & GGG & A \\
\hline Met -16 & $\mathrm{TT}$ & GGG & TTA & GGG & TTA & GMG & TTA & GGG & A \\
\hline Met -17 & $\mathrm{TT}$ & GGG & TTA & GGG & TTA & GGM & TTA & GGG & A \\
\hline Met -21 & $\mathrm{TT}$ & GGG & TTA & GGG & TTA & GGG & TTA & MGG & A \\
\hline Met -22 & $\mathrm{TT}$ & GGG & TTA & GGG & TTA & GGG & TTA & GMG & A \\
\hline Met -23 & $\mathrm{TT}$ & GGG & TTA & GGG & TTA & GGG & TTA & GGM & A \\
\hline Hex-3 & $\mathrm{TT}$ & HGG & TTA & GGG & TTA & GGG & TTA & GGG & A \\
\hline Hex -4 & $\mathrm{TT}$ & GHG & TTA & GGG & TTA & GGG & TTA & GGG & A \\
\hline Hex -5 & $\mathrm{TT}$ & GGH & TTA & GGG & TTA & GGG & TTA & GGG & A \\
\hline Hex -9 & $\mathrm{TT}$ & GGG & TTA & HGG & TTA & GGG & TTA & GGG & A \\
\hline Hex -10 & $\mathrm{TT}$ & GGG & TTA & GHG & TTA & GGG & TTA & GGG & A \\
\hline Hex -11 & $\mathrm{TT}$ & GGG & TTA & GGH & TTA & GGG & TTA & GGG & A \\
\hline Hex -15 & $\mathrm{TT}$ & GGG & TTA & GGG & TTA & HGG & TTA & GGG & A \\
\hline Hex -16 & $\mathrm{TT}$ & GGG & TTA & GGG & TTA & GHG & TTA & GGG & A \\
\hline Hex -17 & $\mathrm{TT}$ & GGG & TTA & GGG & TTA & GGH & TTA & GGG & A \\
\hline Hex -21 & $\mathrm{TT}$ & GGG & TTA & GGG & TTA & GGG & TTA & HGG & A \\
\hline Hex -22 & $\mathrm{TT}$ & GGG & TTA & GGG & TTA & GGG & TTA & GHG & A \\
\hline Hex -23 & $\mathrm{TT}$ & GGG & TTA & GGG & TTA & GGG & TTA & GGH & A \\
\hline Ben-3 & $\mathrm{TT}$ & BGG & TTA & GGG & TTA & GGG & TTA & GGG & A \\
\hline Ben-4 & $\mathrm{TT}$ & GBG & TTA & GGG & TTA & GGG & TTA & GGG & A \\
\hline Ben-5 & $\mathrm{TT}$ & GGB & TTA & GGG & TTA & GGG & TTA & GGG & A \\
\hline Ben-9 & $\mathrm{TT}$ & GGG & TTA & BGG & TTA & GGG & TTA & GGG & A \\
\hline Ben-10 & $\mathrm{TT}$ & GGG & TTA & GBG & TTA & GGG & TTA & GGG & A \\
\hline Ben-11 & $\mathrm{TT}$ & GGG & TTA & GGB & TTA & GGG & TTA & GGG & A \\
\hline Ben-15 & $\mathrm{TT}$ & GGG & TTA & GGG & TTA & BGG & TTA & GGG & A \\
\hline Ben-16 & $\mathrm{TT}$ & GGG & TTA & GGG & TTA & GBG & TTA & GGG & A \\
\hline Ben-17 & $\mathrm{TT}$ & GGG & TTA & GGG & TTA & GGB & TTA & GGG & A \\
\hline Ben-21 & $\mathrm{TT}$ & GGG & TTA & GGG & TTA & GGG & TTA & BGG & A \\
\hline Ben-22 & TT & GGG & TTA & GGG & TTA & GGG & TTA & GBG & A \\
\hline Ben-23 & $\mathrm{TT}$ & GGG & TTA & GGG & TTA & GGG & TTA & GGB & A \\
\hline
\end{tabular}

anti glycosidic conformations. The diverse structural features of this $(3+1)$ folding topology provide an ideal scaffold to test the functionalization of G-quadruplex nanoparticles through N2-modifications.

The N2-modifications tested in this study were chosen to explore a variety of functionalgroup geometries. They include small $\left({ }^{\mathrm{Met}} \mathrm{G}\right)$, bulky-aromatic $\left({ }^{\mathrm{Ben}} \mathrm{G}\right)$ and long chain-like 
$\left({ }^{\text {Hex }} \mathrm{G}\right)$ chemistries. The ability of modified sequences to form G-quadruplex nanoparticles was evaluated by monitoring the imino proton region of ${ }^{1} \mathrm{H}$ NMR spectra and by CD spectroscopy. G-quadruplex nanoparticles were studied in a variety of salt concentrations including "low salt” (1 mM KPi with $1 \mathrm{mM} \mathrm{KCl),} \mathrm{“moderate} \mathrm{salt”} \mathrm{(10} \mathrm{mM} \mathrm{KPi} \mathrm{with} 10$ $\mathrm{mM} \mathrm{KCl})$ and "physiological salt" (10 mM KPi with $130 \mathrm{mM} \mathrm{KCl)} \mathrm{environments} \mathrm{in} \mathrm{order}$ to aid in the comparison of thermal stabilities of different constructs and to investigate nanoparticle formation in different salt concentrations (Appendix II - Protocols - Chapter 7).

Our study explored a total of 36 sequences containing single-position N2-modifications (Table 7-1). All modified sequences demonstrated G-quadruplex formation as determined by the presence of distinct peaks in the imino proton region of NMR spectra. Of these sequences, 30 were observed to fold into a single major species and adopted the same $(3+1)$ conformation as the parent sequence based on similarity of NMR and CD spectra (Fig 7-2 and Table 7-2). This included 12 of the 12 sequences tested for the ${ }^{\mathrm{Met}} \mathrm{G}$ chemistry, 8 for ${ }^{\mathrm{Hex}} \mathrm{G}$, and 10 for ${ }^{\mathrm{Ben}} \mathrm{G}$. Classification by groove type revealed that modified sequences containing substitution into the $N, P, W$ and $M$ grooves maintained the $(3+1)$ conformation in $6,7,8$, and 9 sequences, respectively, out of the 9 modifications that were tested per groove. Select N2-modifications were also tested in a parallel propeller type G-quadruplex scaffold, with G-quadruplex formation observed via imino proton NMR spectra (Fig 7-3), suggesting that N2-guanine modifications can be successfully incorporated into a variety of G-quadruplex structures.

The combination of NMR and CD data suggest that N2-modifications do not inhibit Gquadruplex formation, as all sequences were observed to adopt G-quadruplex structures. As hypothesized, most N2-modified guanines substitutions were successfully introduced 


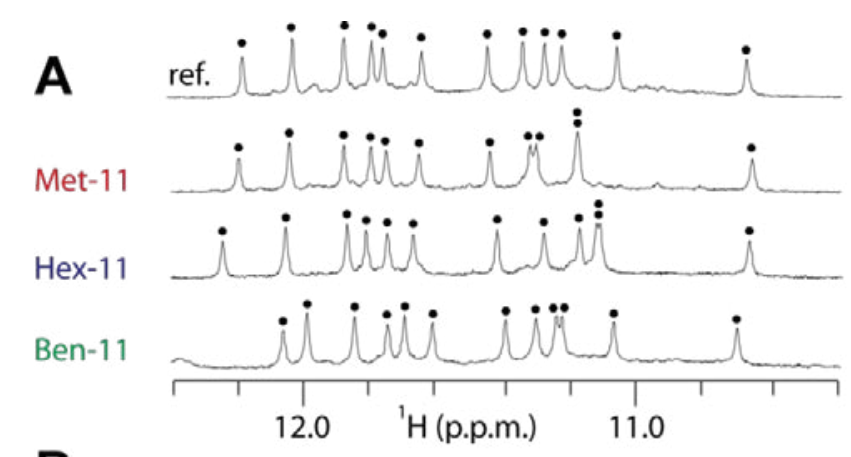

B

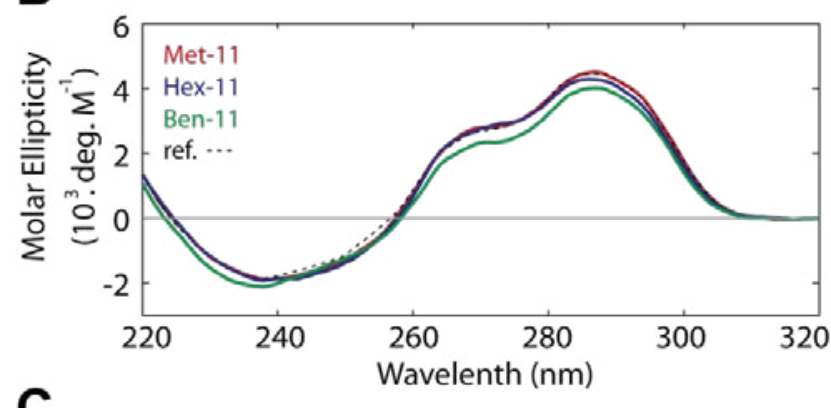

C

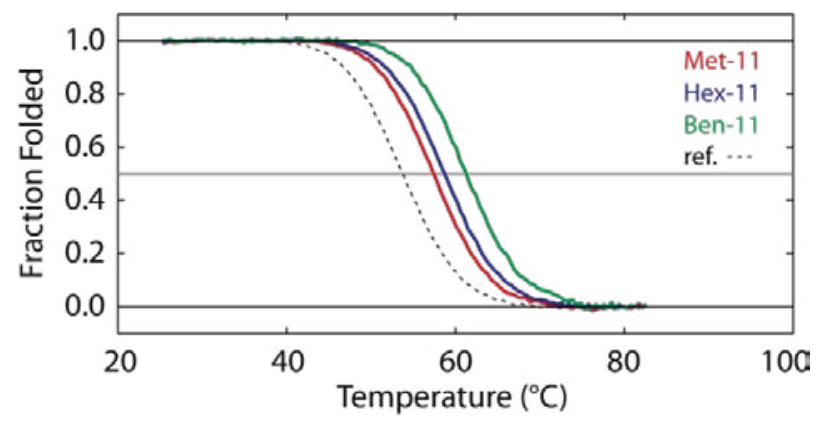

Figure 7-2: G-quadruplex nanoparticles containing single N2modifications: (A) NMR and (B) CD spectra of representative N2modified sequences for each of the ${ }^{\text {Met }} G$, ${ }^{\text {Hex }} G$, and ${ }^{\text {Ben }} G$ chemistries that maintained the original $(3+1)$ folding topology. Each imino proton resonances in (A) is indicated by a dot $(\bullet)$ (C) Melting curves of these sequences observed by UV absorbance at $295 \mathrm{~nm}$ in "moderate salt" conditions (10 mM KPi with $10 \mathrm{mM} \mathrm{KCl})$. Spectra of the unmodified sequence are shown for reference.

without perturbing the native $(3+1)$ conformation. The smaller ${ }^{\mathrm{Met}} \mathrm{G}$ substitutions are less disruptive to the native $(3+1)$ conformation than ${ }^{B e n} G$ or ${ }^{\text {Hex }} G$ substitutions. It is not surprising that modifications into the $N$ or $P$ grooves were more disruptive to the native G-quadruplex conformation than those into $M$ and $W$ grooves, as modification into the $M$ and $W$ grooves are expected to be less obstructed. We note that N2-modified guanine substitutions into positions 5 and 16 of the $(3+1)$ hybrid G-quadruplex are not well tolerated, with ${ }^{\text {Hex }} G$ and ${ }^{\text {Ben }} G$ modifications inducing a mixture of conformations and ${ }^{\text {Met }} G$ 
Table 7-2: Biophysical characterization of sequences

\begin{tabular}{|c|c|c|c|}
\hline \multirow[b]{2}{*}{ Sequence } & \multicolumn{3}{|c|}{ NMR $^{a}$} \\
\hline & $\begin{array}{c}\text { Single } \\
\text { Major } \\
\text { Species }\end{array}$ & $\begin{array}{c}\text { Notable } \\
\text { Minor } \\
\text { Population }\end{array}$ & $\begin{array}{c}\text { Multiple } \\
\text { Major } \\
\text { Species }\end{array}$ \\
\hline Unmodified & • & & \\
\hline Met-3 & - & & \\
\hline Met-4 & • & & \\
\hline Met-5 & & • & \\
\hline Met-9 & $\bullet$ & & \\
\hline Met-10 & • & & \\
\hline Met-11 & • & & \\
\hline Met-15 & - & & \\
\hline Met-16 & & $\bullet$ & \\
\hline Met-17 & $\bullet$ & & \\
\hline Met-21 & & - & \\
\hline Met-22 & • & & \\
\hline Met-23 & & $\bullet$ & \\
\hline Hex-3 & $\bullet$ & & \\
\hline Hex-4 & $\bullet$ & & \\
\hline Hex-5 & & & - \\
\hline Hex-9 & • & & \\
\hline Hex-10 & & & - \\
\hline Hex-11 & - & & \\
\hline Hex-15 & - & & \\
\hline Hex-16 & & & • \\
\hline Hex-17 & $\bullet$ & & \\
\hline Hex-21 & & & • \\
\hline Hex-22 & • & & \\
\hline Hex-23 & • & & \\
\hline Ben-3 & & $\bullet$ & \\
\hline Ben-4 & - & & \\
\hline Ben-5 & & & $\bullet$ \\
\hline Ben-9 & - & & \\
\hline Ben-10 & • & & \\
\hline Ben-11 & • & & \\
\hline Ben-15 & $\bullet$ & & \\
\hline Ben-16 & & & • \\
\hline Ben-17 & & - & \\
\hline Ben-21 & - & & \\
\hline Ben-22 & - & & \\
\hline Ben-23 & & - & \\
\hline
\end{tabular}

[a] Determination of the same (3+1) conformation being adopted is based off a qualitative analysis of the amount of minor conformation present in NMR spectra and the similarity of NMR and CD spectra between the modified and unmodified sequences.

[b] The \pm values indicated the hysteresis observed between heating and cooling curved in UV melting experiments

modifications producing a minor species in NMR spectra. Upon examination of the NMR solution structure of the native sequence, it is possible that modification into position 5 may clash with the propeller loop situated in that groove. There is no clear structural explanation for why position 16 is not well tolerated. It is important to note that multiple 

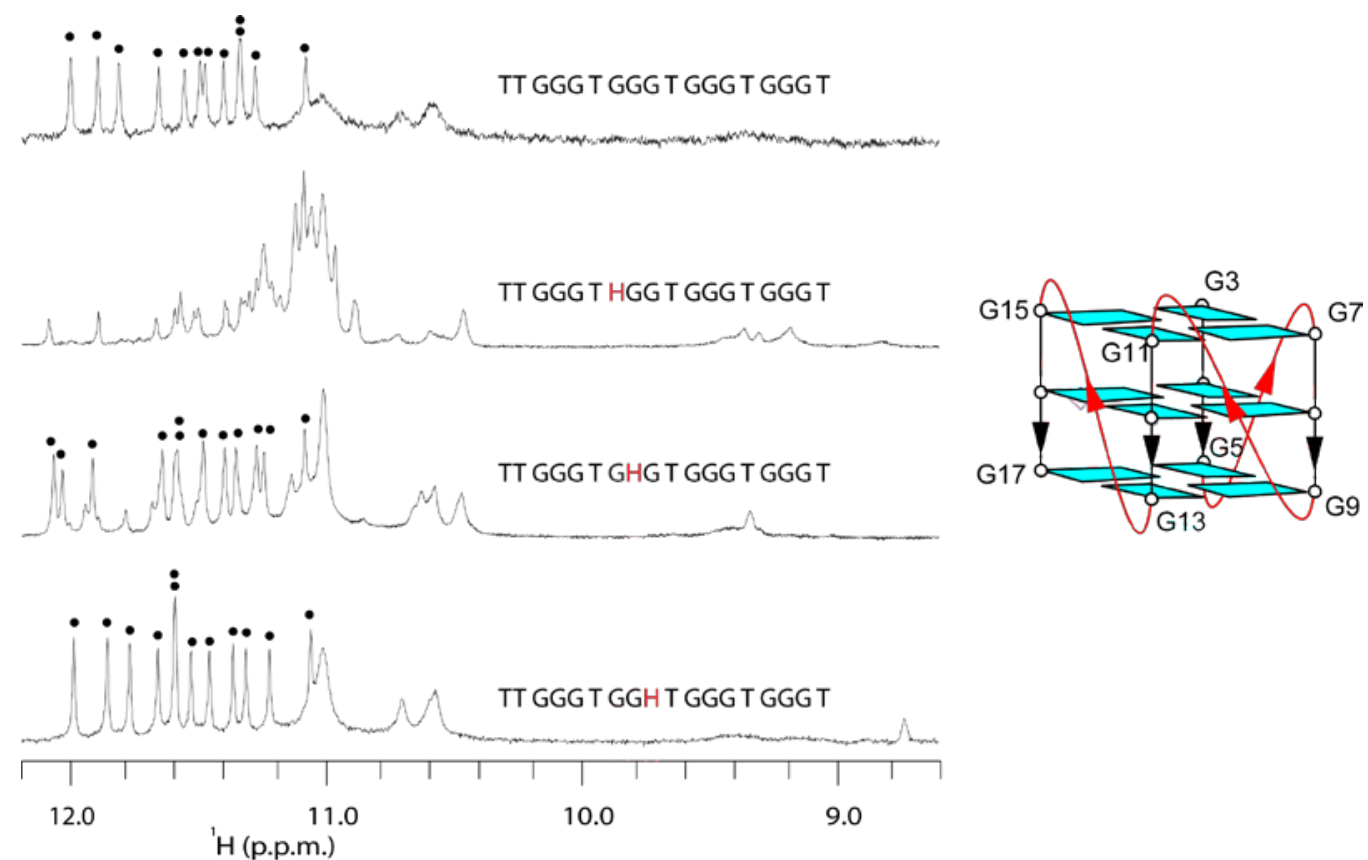

Figure 7-3: ${ }^{1} \mathrm{H}$ NMR spectra of sequences containing single ${ }^{\mathrm{Hex}} \mathrm{G}$ modifications to the sequence $\mathrm{d}\left[\mathrm{T}_{2}(\mathrm{G} 4 \mathrm{~T})_{4}\right]$. The position of ${ }^{\mathrm{Hex}} \mathrm{G}$ modification is indicated in the sequence by a red "H". Black dots indicate imino proton resonances of the monomer $\mathrm{G}$-quadruplex.

species in solution can arise from the destabilization of the $(3+1)$ structure and/or from the stabilization of an alternative G-quadruplex structure.

\subsection{2: N2-modified G-quadruplexes display enhanced thermal stability}

We proceeded to investigate how N2-guanine modifications affect the thermal stability of G-quadruplex nanoparticles in a "moderate salt” environment. We examined the stability of all sequences that maintained the native $(3+1)$ G-quadruplex structure. Thermal denaturing studies were performed to determine the melting temperature $\left(T_{m}\right)$ of the unmodified and N2-modified sequences by monitoring the $295 \mathrm{~nm}$ UV absorption as a function of temperature (258). Remarkably, all sequences that maintained the original (3+1) G-quadruplex conformation demonstrated enhanced thermal stability (Table 7-3), with a change in melting temperature $\left(\Delta \mathrm{T}_{\mathrm{m}}\right)$ observed in the range of $+2.0^{\circ} \mathrm{C}$ to $+13.2^{\circ} \mathrm{C}$ for each single-modification. Average $\Delta \mathrm{T}_{\mathrm{m}}$ values of $4.6^{\circ} \mathrm{C}, 6.0^{\circ} \mathrm{C}$, and $6.6^{\circ} \mathrm{C}$ were obser- 
Table 7-3. Change in melting temperature $\left({ }^{\circ} \mathrm{C}\right)$ upon single-position N2-modifications

\begin{tabular}{ccccc}
\hline $\begin{array}{c}\text { Modification } \\
\text { Position }\end{array}$ & Groove ${ }^{\text {[a] }}$ & Met & Hex & Ben \\
\hline 3 & $M$ & 5.3 & 9.1 & 6.2 \\
4 & $P$ & 5.7 & 8.2 & 8.5 \\
5 & $P$ & 4.0 & - & - \\
9 & $P$ & 8.3 & 9.1 & 13.2 \\
10 & $W$ & 4.1 & - & 6.9 \\
11 & $W$ & 3.4 & 4.6 & 7.4 \\
15 & $N$ & 5.5 & 4.4 & 3.0 \\
16 & $N$ & 3.1 & - & - \\
17 & $W$ & 2.8 & 2.0 & 4.5 \\
21 & $N$ & 5.2 & - & 7.9 \\
22 & $M$ & 5.4 & 7.8 & 5.1 \\
23 & $M$ & 2.5 & 3.1 & 2.8 \\
\hline "Moderate salt” conditions were used (10 mM KPi with 10 mM KCl).
\end{tabular}

[a] The notation describing groove geometry is as shown in Figure 7-1

ved upon modification with ${ }^{\mathrm{Met}} \mathrm{G}$, ${ }^{\mathrm{Hex}} \mathrm{G}$, and ${ }^{\mathrm{Ben}} \mathrm{G}$ respectively. Further classification of $\Delta \mathrm{T}_{\mathrm{m}}$ values by groove type reveal average $\Delta \mathrm{T}_{\mathrm{m}}$ values of $8.1^{\circ} \mathrm{C}, 5.3^{\circ} \mathrm{C}, 4.9^{\circ} \mathrm{C}$, and $4.5^{\circ} \mathrm{C}$ for the $P, M, N$, and $W$ grooves respectively.

The highly-stabilizing nature of N2-guanine modification into G-quadruplex is an important finding of this work. The extent of stabilization is chemistry-dependent, with the N2-modified guanines tested here ranked as ${ }^{B e n} G>{ }^{\text {Hex }} G>{ }^{\text {Met }} G$. Stabilizing effects are also position-dependent as modifications to certain residues, 4 and 9 for example, are observed to be highly stabilizing while other positions, such as 17 and 23, show moderate $\mathrm{T}_{\mathrm{m}}$ enhancement. It was unexpected that N2-modifications into positions 4 and 9 of the $P$ groove yielded some of the most stable structures, as modifications at these positions could potentially clash with the loop occupying the groove. Conversely, substitutions into the $W$ groove at position 17 yielded some of the most modestly stabilized sequences. These observations suggest that the stabilizing properties of N2-modifications may be due to favorable interactions with neighboring structural elements, including the neighboring sugar-phosphate backbone. It is important to note that modifications replacing one of the amino protons with a bulkier chemistry should freeze the rotation about the N2-position, 
as supported by the emergence of sharp peaks in the region of 9.0-10.0 ppm in NMR spectra of most modified sequences.

\subsection{3: Design of highly stabilized G-quadruplex nanoparticles}

The stabilizing properties of single N2-guanine modifications into the $(3+1) \mathrm{G}$ quadruplex scaffold led us to pursue the design of highly stabilized G-quadruplex nanoparticles. We suspected that multiple N2-guanine substitutions would further enhance the stability of the molecular scaffold while simultaneously generating multiple sites to potentially functionalize the molecule. A number of sequences were synthesized containing a variety of N2-modifications in numerous positions based on the results of our single substitution studies (Table 7-4).

We designed a sequence "N2-Same-Groove" containing two ${ }^{\mathrm{Met}} \mathrm{G}$ modifications into the $W$ groove at position 10 and 11 to examine if multiple N2-modified guanines could be substituted at neighboring positions within the same groove. The sequence "N2-DifferentGroove” tests the incorporation of two very stabilizing modifications into two separate grooves, with two ${ }^{\mathrm{Hex}} \mathrm{G}$ substitutions into the $M$ and $P$ grooves at positions 3 and 9 . We

Table 7-4. Thermal stability of sequences containing multiple N2-modifications

\begin{tabular}{|c|c|c|c|}
\hline & Sequence ${ }^{\text {[a] }}$ & $\mathrm{T}_{\mathrm{m}}{ }^{\mathrm{b}}$ & $\Delta \mathrm{T}_{\mathrm{m}}$ \\
\hline Unmodified & TT GGG TTA GGG TTA GGG TTA GGG A & $46.3 \pm 0.3$ & - \\
\hline N2-One-Groove & TT GGG TTA GMM TTA GGG TTA GGGA & $53.4 \pm 1.0$ & 7.2 \\
\hline N2-Two-Groove & TT HGG TTA HGG TTA GGG TTA GGG A & $65.2 \pm 0.4$ & 18.9 \\
\hline N2-All-Groove & TT $\mathrm{H}$ GG TTA $\mathrm{H}$ M TTA MGG TTA GGG A & $73.8 \pm 0.2$ & 27.6 \\
\hline N2-Ball-with-Hair & TT $\mathrm{HH} G$ TTA $\mathrm{H} G \mathrm{H}$ TTA & $94.0 \pm 0.1^{\mathrm{c}}$ & 47.8 \\
\hline N2-SS8 & TT $\mathrm{H} G \mathrm{G}$ TTA BG TTA $\mathrm{M}$ G TTA BGB A & $85.0 \pm 0.6$ & 38.7 \\
\hline N2-SS10 & TT HBG TTA BMB TTA M G & $91.9 \pm 0.2^{\mathrm{d}}$ & 45.6 \\
\hline \multicolumn{4}{|c|}{$\begin{array}{l}\text { Samples were examined in "Low Salt" conditions (1 mM KPi with } 1 \mathrm{mM} \mathrm{KCl).} \\
\text { [a] The position of N2-modified guanines is boxed with the following notation: " } \mathrm{M} \text { “ for }{ }^{\mathrm{Met}} \mathrm{G} \text {; " } \mathrm{H} \text { “ for }{ }^{\mathrm{Hex}} \mathrm{G} \text {; "B" for }{ }^{\mathrm{Ben}} \mathrm{G} \text {. } \\
\text { [b] } \pm \text { indicate the hysteresis between heating and cooling curves in CD melting experiments } \\
\text { [c] Incomplete melting transition observed. Tm determined by single base line correction with high temperature baseline set } \\
\text { to a constant of } \approx 0 \text { absorbance. } \\
\text { [d] Incomplete melting transition observed. Tm determined by } 50 \% \text { of maximum signal due to lack of a low temperature } \\
\text { baseline. }\end{array}$} \\
\hline
\end{tabular}


design a sequence "N2-Same-Groove" containing two ${ }^{\mathrm{Met}} \mathrm{G}$ modifications into the $W$ groove at position 10 and 11 to examine if multiple N2-modified guanines could be substituted at neighboring positions within the same groove. The sequence "N2-DifferentGroove” tests the incorporation of two very stabilizing modifications into two separate grooves, with two ${ }^{\text {Hex }} \mathrm{G}$ substitutions into the $M$ and $P$ grooves at positions 3 and 9 respectively. A substitution of one N2-modified guanine per groove was investigated by two ${ }^{\text {Hex }} \mathrm{G}$ modifications at positions 3 and 9 and two ${ }^{\text {Met }} \mathrm{G}$ substitutions at 11 and 15 in the sequence "N2-All-Groove". We also pursued a more ambitious design in the sequence “N2-Ball-with-Hair” containing eight ${ }^{\mathrm{Hex}} \mathrm{G}$ modifications into positions 3, 4, 9, 11, 15, 17 , 22 and 23. The "Ball-with-Hair" nanoparticle is designed to contain many long and flexible N2-hexyl-chemistries capable of extending outwards from the surface of the Gquadruplex offering potential sites for functionalization. In addition, we report on the design of N2-SS8 and N2-SS10 sequences containing multiple N2-modifications.

Designed sequences were tested in a "low salt" solution in order to lower the $T_{m}$ values, maximizing the range of $T_{m}$ values that could be observed for comparing the stability of different constructs. The designed sequences described in Table 7-4 were determined to maintain the $(3+1)$ G-quadruplex conformation by analysis of ${ }^{1} \mathrm{H}$ NMR and CD spectra, which were highly similar to the parent sequence (Fig. 7-4A-B). The thermal stability of designed sequences was examined through melting experiments by monitoring the CD signal at $290 \mathrm{~nm}$ (270). As desired, all of the sequences tested containing multiple N2modifications displayed highly increased thermal stability (Fig 7-4C). The N2-OneGroove, N2-Two-Groove, and N2-All-Groove sequences displayed $\Delta \mathrm{T}_{\mathrm{m}}$ values of $+7.2^{\circ} \mathrm{C}$, $+18.9^{\circ} \mathrm{C}$ and $+27.6^{\circ} \mathrm{C}$ respectively, roughly equivalent to the sum of $\Delta \mathrm{T}_{\mathrm{m}}$ values gained from individual modifications. The N2-Ball-with-Hair sequence demonstrated the highest thermal stability, with a $\Delta \mathrm{T}_{\mathrm{m}}$ of $+47.8^{\circ} \mathrm{C}$ and a melting temperature of $\sim 94^{\circ} \mathrm{C}$. 

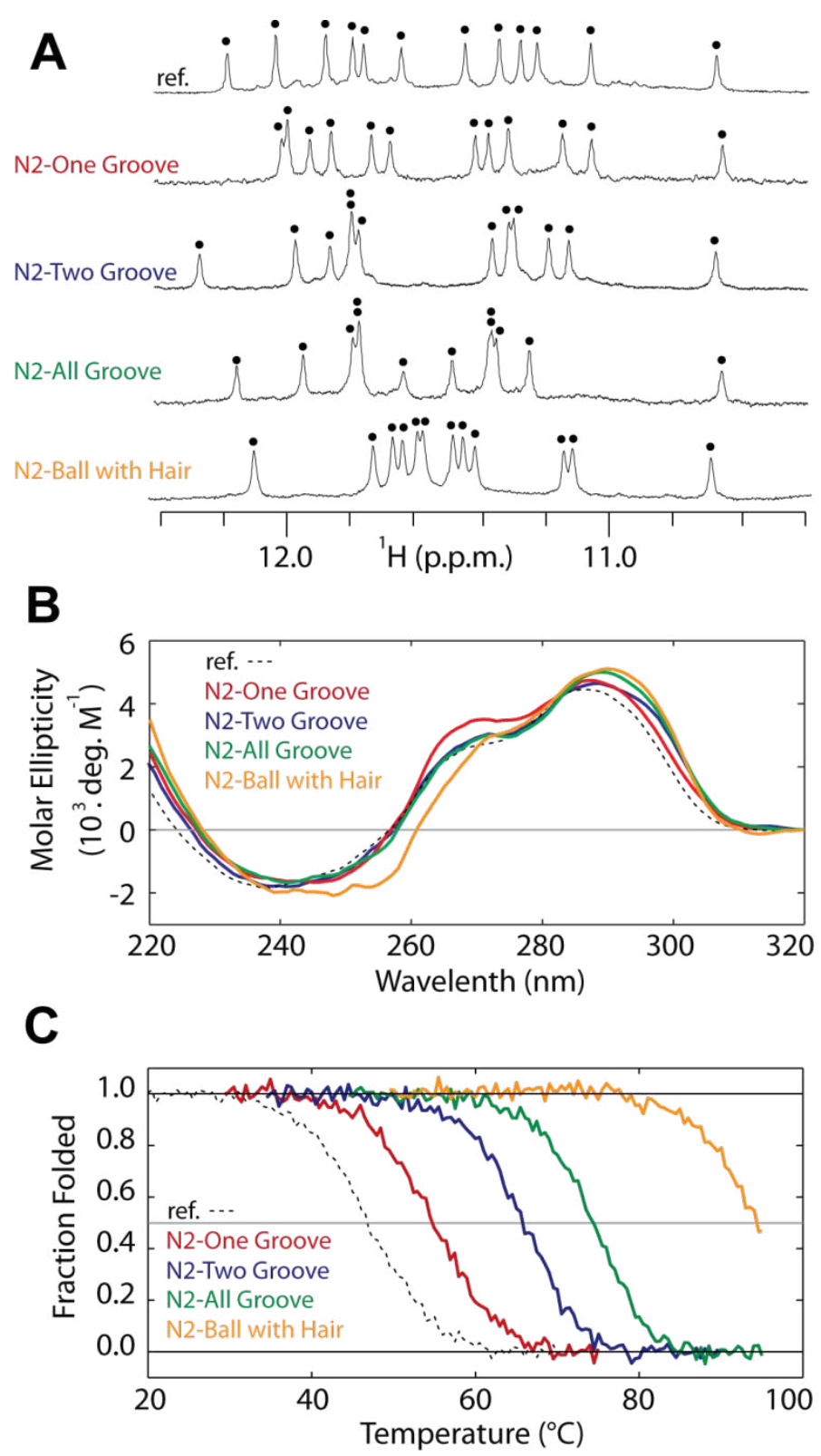

Figure 7-4: Design of highly stable G-quadruplex nanoparticles containing multiple N2-modifications: (A) NMR and (B) CD spectra of select nanoparticles including the N2-Ball with Hair construct (orange). Each imino proton resonances in $(\mathbf{A})$ is indicated with a dot $(\bullet)$. (C) Melting curves of designed sequences observed by CD at $290 \mathrm{~nm}$ in "low salt" (1 mM KPi with $1 \mathrm{mM} \mathrm{KCl)} \mathrm{conditions.} \mathrm{Spectra} \mathrm{of} \mathrm{the}$ unmodified sequence are shown for reference.

We further tested the stability of the N2-Ball-with-Hair sequence in "physiological salt" conditions similar to those experienced in a cellular environment. Temperature dependent CD spectra show more than $80 \%$ signal intensity observed at $95^{\circ} \mathrm{C}$ (Fig $7-5$ ), suggesting the "Ball-with-Hair" G-quadruplex nanoparticle is highly stable in physiological $\mathrm{K}^{+}$ conditions. 


\section{A}

\section{N2-Ball-with-Hair}
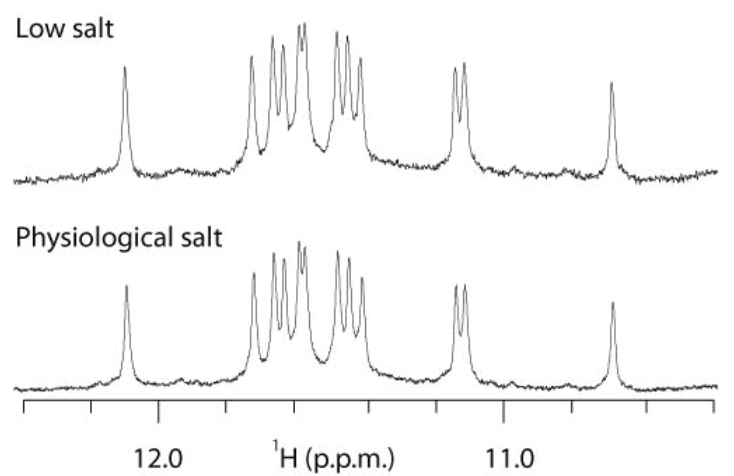

B
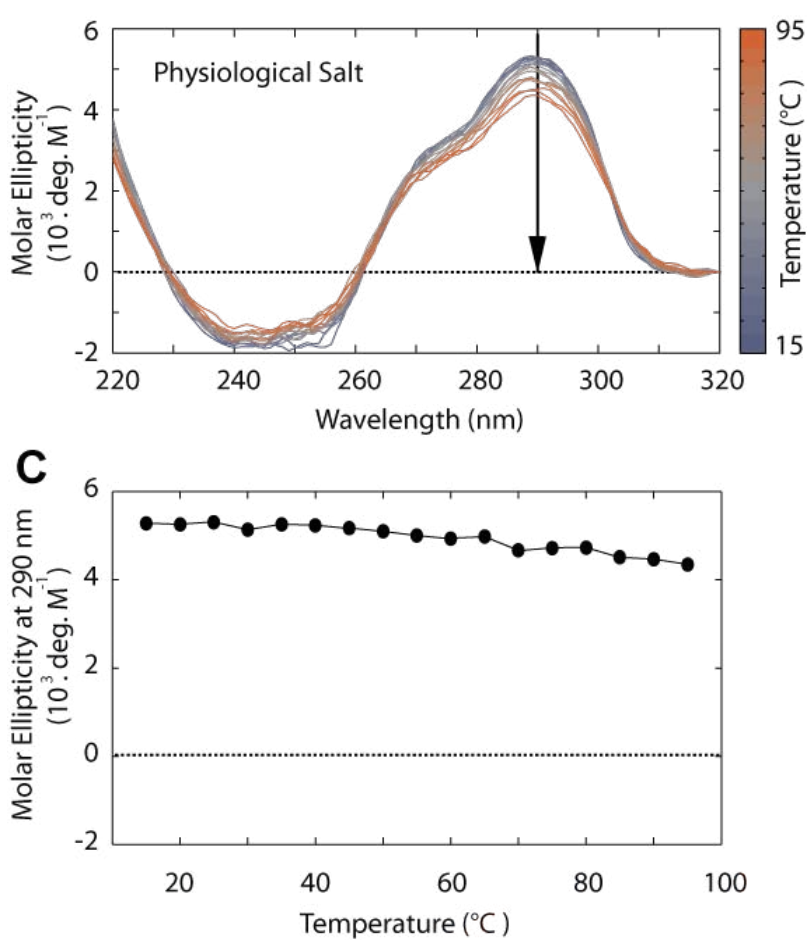

Figure 7-5: Design of a ${ }^{\mathrm{Hex}} \mathrm{G}$ modified "Ball-with-hair" G-quadruplex nanoparticle: (A) NMR spectra of N2-Ball-with-Hair sequence in "low" and "physiological" salt conditions shows the same structure is formed. (B) Temperature dependent CD spectra demonstrate the incredible stability of the "Ball-with-hair" nanoparticle in physiological salt conditions. (C) Projection of CD intensity at $290 \mathrm{~nm}$ over varying temperatures.

The ability of multiple N2-modifications to stabilize the G-quadruplex without disrupting the folding topology demonstrates the great potential of these tools. Such chemistries can be used to decorate the G-quadruplex scaffold in a variety of ways, including a Ball-withHair-like fashion allowing multiple sites for functionalization (Fig 7-6). Previous studies have shown that modifications by ${ }^{\mathrm{Met}} \mathrm{G}$ or other bulky N2-modifications can enhance the 

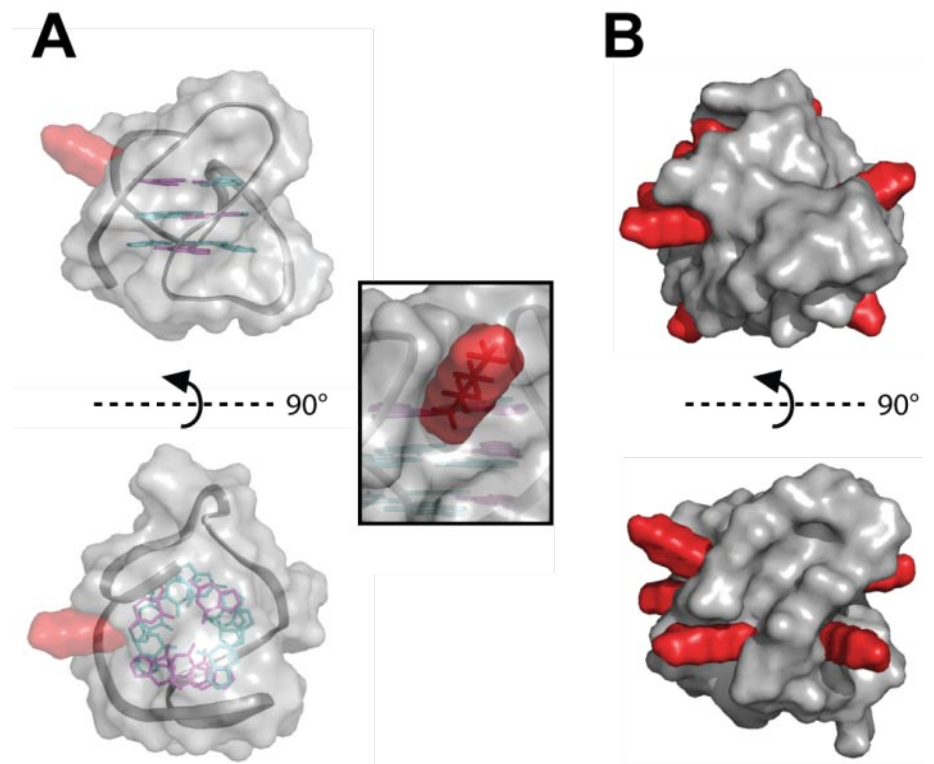

Figure 7-6: Design of a ${ }^{\mathrm{Hex}} \mathrm{G}$ modified "Ball-with-hair” G-quadruplex nanoparticle: (A) Surface representation of G-quadruplex molecule (grey) modified with a N2-6-amino-hexyl group (red) in the position G3 illustrates the location and dimension of the ${ }^{\text {Hex }} \mathrm{G}$ modification with respect to the $M$ groove. (B) Illustrative model of the "Ball with Hair" G-quadruplex nanoparticle formed by the N2-Ball with Hair sequence containing eight ${ }^{\mathrm{Hex}} \mathrm{G}$ modifications.

inhibitory properties of biologically active G-quadruplex based drugs (122,123), demonstrating the value of N2-modifications in pharmacologically relevant Gquadruplexes. Additionally, the modification of an entire tetrad by ${ }^{\text {Met }} \mathrm{G}$ substitutions has been reported to stabilize a tetramer G-quadruplex with anti-HIV activity (123). 


\subsection{Conclusions:}

Our current research reports on the versatility of N2-modifications in a structurally diverse $(3+1)$ G-quadruplex scaffold. Our results indicate that single substitutions of N2modified guanines are potent stabilizers of the G-quadruplex architecture and we show the ability to decorate this molecular scaffold with a variety of chemistries, including long chain-like chemistries that can be used as a bridge to functionalize this molecular scaffold. Experimentally, we design a "Ball with Hair" type nanoparticle through the addition of eight ${ }^{\mathrm{Hex}} \mathrm{G}$ modifications to our 3-layered G-quadruplex scaffold yielding a remarkable increase in thermal stability of $\sim 48^{\circ} \mathrm{C}$ without disrupting the conformation of the Gquadruplex nanoparticle. Our study demonstrates the design of highly stable Gquadruplex based nanoparticle systems, which are efficiently self-folding, ion sensitive, easily synthesized, and functionalizeable in a spatially-specific manner. 


\section{Chapter 8:}

\section{Summary and Future Work}

\subsection{Summary}

We have demonstrated that unique G-tetrad stacking geometries exists within the core and at the stacking interface of G-quadruplex DNA. The geometries of interfacial G-tetrad orientations from experimental structures are estimated to be energetically different by up to $12 \mathrm{kcal} / \mathrm{mol}$. We show that current MM force field, popularly used for simulations of nucleic acid, well-represents the relative interaction of stacked G-tetrad systems. MD simulations of parallel G-quadruplex systems demonstrate different accessible G-tetrad geometries for 5'-5' and 3'-3' stacked orientations. These results suggest favorability of G-tetrad orientations may be a key driver of the experimentally observed preference of Gquadruplexes to stack in a 5' -5' manner.

The ground state electronic properties of stacked G-tetrads and vary greatly depending on their stacking geometry and thus the geometry adopted by stacked guanines bases. We demonstrate the 5/6-ring stacking mode commonly found at the interface between 5' 5 ' stacked G-quadruplex systems is far superior to other stacking orientations in both the 
extent of charge delocalization and speed of charge propagation through the system. Electron hole transfer rates for this geometry were at least an order of magnitude greater than other geometries explored.

Incorporation of LNA-modified guanosine $\left({ }^{\mathrm{LNA}} \mathrm{G}\right)$ into G-quadruplex was found to generally be tolerated based on the glycosidic conformation of the base, with antiposition substitutions being tolerated and syn-position substitutions perturbing the native (3+1) G-quadruplex fold. However, we indentify a scenario of both a syn-tolerated and anti-destabilizing ${ }^{\mathrm{LNA}} \mathrm{G}$ modification. We discover a novel limitation of ${ }^{\mathrm{LNA}} \mathrm{G}$ incorporation, with substitution prior to short loops being highly deleterious to Gquadruplex formation. The High-resolution NMR structure of a $(3+1)$ G-quadruplex

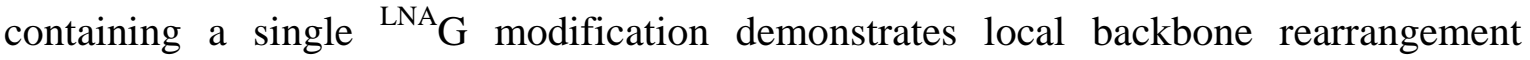
upstream of the nucleotide suggesting a possible structural mechanism for the incompatibility of ${ }^{\mathrm{LNA}} \mathrm{G}$ modification prior to short loops.

We report on the versatile nature of 2'-deoxy-2'-fluoro-riboguanosine ( ${ }^{\mathrm{F}} \mathrm{G}$ ) and 2'-deoxy-

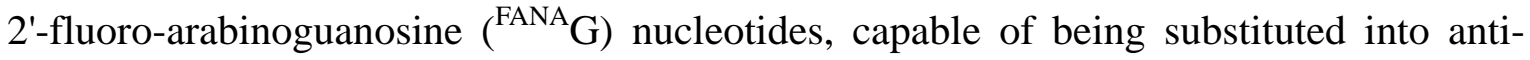
conformation guanines in many structural environments without disrupting the Gquadruplex folding topology. This is in contrast to ${ }^{\mathrm{LNA}} \mathrm{G}$ which has limitations. ${ }^{\text {FANA }} \mathrm{G}$ demonstrates stabilizing properties while ${ }^{\mathrm{F}} \mathrm{G}$ demonstrates a mixture of modestly stabilizing and destabilizing properties. Incorporation of both of these nucleotides allows for the isolation of specific folding topologies from a mixture of conformers, or allows for the complete alteration of G-quadruplex folding topology in a polymorphic sequence.

The C8-modified chemistries 8-bromo-guanine $\left({ }^{\mathrm{Br}} \mathrm{G}\right)$, 8-O-methyl-guanine ( $\left.{ }^{\mathrm{Omet}} \mathrm{G}\right)$, 8amino-guanine $\left({ }^{\mathrm{Am}} \mathrm{G}\right)$, and 8-oxo-guanine $\left({ }^{\mathrm{Oxo}} \mathrm{G}\right)$ demonstrate a preference for syn- 
position substitutions. Furthermore, we demonstrate the site-specific and chemicalspecific nature of these modifications. Generally, modifications may be ranked ${ }^{\mathrm{Br}} \mathrm{G}>$ ${ }^{\text {Omet }} \mathrm{G}>{ }^{\mathrm{Am}} \mathrm{G}$ in their ability to stabilizing the G-quadruplex scaffold. ${ }^{\text {Oxo }} \mathrm{G}$ substitutions are observed to be deleterious to G-quadruplex formation. The modification-induced change in NMR chemical shift of modified G-tetrad is reproduced using QM methods. The up-field shifting (decreased p.p.m) of imino proton resonances correspond to an increase in hydrogen bond length and vice versa.

The N2-modifications are shown to be highly-stabilizing with a single modification stabilizing between $2 \cdot 0-13 \cdot 2^{\circ} \mathrm{C}$ degrees. Multiple substitutions allow the design of a Gquadruplex based nanoparticle with an increased thermal stability of $+50^{\circ} \mathrm{C}$ and a melting temperature of $>95^{\circ} \mathrm{C}$ in physiological salt conditions. This designed nanoparticle contains multiple sites for functionalization. We present this engineered "Ball-with-Hair" G-quadruplex-based nano-scaffold as a useful tool with a variety of potential applications. 


\subsection{Future Work}

Our computational studies of G-tetrad stacks have demonstrated large variability in the energy and electronic properties of different stacking orientations. Recent work from our laboratory and others has shown that the florescence emission of G-quadruplexes is enhanced and red-shifted by the stacking of individual G-quadruplex units, but only for certain sequences (271). Knowledge of the G-tetrad stacking orientations at the interface of these dimers leads us to believe that G-tetrad stacking geometry may play a large role in the florescent properties of these molecules. With this in mind, we plan to initiate a computational investigation of the excited state properties of different G-tetrad stacking orientations. A better understanding of how G-tetrad geometry affects excited state properties could aid in the designing G-quadruplex-based sensors and conductive devices.

Our work on alternative nucleic acid chemistries has enabled a better understanding of how to effectively apply guanosine analogs into the G-tetrad core of the G-quadruplex. We demonstrate that sugar-modified guanines ${ }^{\mathrm{LNA}} \mathrm{G}$, and ${ }^{\text {FANA }} \mathrm{G}$ are generally potent stabilizers of anti-position guanines. Alternatively, C8-modified guanine bases are potent syn-position stabilizers. Detailed knowledge of how to effectively incorporate these chemical tools into G-quadruplex nucleic acids will allow the design new folding topologies currently unrealized by conventional chemistries. The engineering of such molecules would be of great structural interest and could enable the building of novel Gquadruplex architectures for nanotechnology applications.

We demonstrate the effective incorporation of non-natural chemistries into Gquadruplexes, including highly-stabilizing N2-modified guanines as a novel method for nanoparticle functionalization. Combining the knowledge from our systematic studies 
presented in within, we can combine different types of guanosine analogs in parallel to engineer G-quadruplexes with enhanced biological activity and novel applications in the area of nanotechnology. From a pharmacological perspective, G-quadruplex based aptamers of biological interest can be designed, with various guanine analogs being incorporates. The effects of these modifications on enzymatic degradation, cellular uptake, and in vivo activity can be investigated. From a nanotechnology perspective, the incorporation of multiple types of guanine analogs will allow us to control the assembly of nanoscale architectures and tune the physical properties of such assemblies. 


\section{Bibliography}

1. Watson, J.D. and Crick, F.H.C. (1953) Molecular structure of nucleic Acids - a structure for deoxyribose nucleic acid. Nature, 171, 737-738.

2. Wilkins, M.H.F., Stokes, A.R. and Wilson, H.R. (1953) Molecular structure of deoxypentose nucleic acids. Nature, 171, 738-740.

3. Franklin, R.E. and Gosling, R.G. (1953) Molecular configuration in sodium thymonucleate. Nature, 171, 740-741.

4. Bang, I. (1910) Examination of the guanyle acid. Biochemische Zeitschrift, 26, 293-311.

5. Gellert, M., Lipsett, M.N. and Davies, D.R. (1962) Helix formation by guanylic acid. Proc Natl Acad Sci USA, 48, 2013-2018.

6. Wang, Y. and Patel, D.J. (1993) Solution structure of the human telomeric repeat d[AG3(T2AG3)3] G-tetraplex. Structure, 1, 263-282.

7. Laughlan, G., Murchie, A.I.H., Norman, D.G., Moore, M.H., Moody, P.C.E., Lilley, D.M.J. and Luisi, B. (1994) The high-resolution crystal-structure of a parallel-stranded guanine tetraplex. Science, 265, 520-524.

8. Smith, F.W. and Feigon, J. (1992) Quadruplex structure of oxytricha telomeric DNA oligonucleotides. Nature, 356, 164-168.

9. Sen, D. and Gilbert, W. (1988) Formation of parallel 4-stranded complexes by guanine-rich motifs in DNA and its implications for meiosis. Nature, 334, 364-366.

10. Patel, D.J., Phan, A.T. and Kuryavyi, V. (2007) Human telomere, oncogenic promoter and 5'-UTR G-quadruplexes: diverse higher order DNA and RNA targets for cancer therapeutics. Nucleic Acids Res, 35, 7429-7455.

11. Paeschke, K., Bochman, M.L., Garcia, P.D., Cejka, P., Friedman, K.L., Kowalczykowski, S.C. and Zakian, V.A. (2013) Pif1 family helicases suppress genome instability at G-quadruplex motifs. Nature, 497, 458-462.

12. Biffi, G., Tannahill, D., McCafferty, J. and Balasubramanian, S. (2013) Quantitative visualization of DNA G-quadruplex structures in human cells. Nat Chem, 5, 182-186.

13. Fry, M. (2007) Tetraplex DNA and its interacting proteins. Front Biosci, 12, 4336-4351.

14. Palm, W. and de Lange, T. (2008) How shelterin protects mammalian telomeres. Annu Rev Genet, 42, 301-334.

15. Zakian, V.A. (1989) Structure and function of telomeres. Annu Rev Genet, 23, 579-604.

16. Blackburn, E.H. (1991) Structure and function of telomeres. Nature, 350, 569-573.

17. Greider, C.W. and Blackburn, E.H. (1985) Identification of a specific telomere terminal transferase activity in Tetrahymena extracts. Cell, 43, 405-413.

18. Greider, C.W. and Blackburn, E.H. (1987) The telomere terminal transferase of tetrahymena is a ribonucleoprotein enzyme with 2 kinds of primer specificity. Cell, 51, 887-898.

19. Autexier, C. and Lue, N.F. (2006) The structure and function of telomerase reverse transcriptase. Annu Rev Biochem, 75, 493-517.

20. Kim, N.W., Piatyszek, M.A., Prowse, K.R., Harley, C.B., West, M.D., Ho, P.L.C., Coviello, G.M., Wright, W.E., Weinrich, S.L. and Shay, J.W. (1994) Specific association of human telomerase activity with immortal cells and cancer. Science, 266, 2011-2015.

21. Parkinson, G.N., Lee, M.P.H. and Neidle, S. (2002) Crystal structure of parallel quadruplexes from human telomeric DNA. Nature, 417, 876-880.

22. Wang, Y. and Patel, D.J. (1992) Guanine residues in D(T2AG3) and D(T2G4) form parallel-stranded potassium cation stabilized G-quadruplexes with antiglycosidic torsion angles in solution. Biochemistry, 31, 8112-8119.

23. Phan, A.T. and Patel, D.J. (2003) Two-repeat human telomeric d(TAGGGTTAGGGT) sequence forms interconverting parallel and antiparallel G-quadruplexes in solution: distinct topologies, thermodynamic properties, and folding/unfolding kinetics. J Am Chem Soc, 125, 15021-15027.

24. Zhang, N., Phan, A.T. and Patel, D.J. $(2005)(3+1)$ Assembly of three human telomeric repeats into an asymmetric dimeric G-quadruplex. J Am Chem Soc, 127, 17277-17285. 
25. Xu, Y., Noguchi, Y. and Sugiyama, H. (2006) The new models of the human telomere d[AGGG(TTAGGG)(3)] in K+ solution. Bioorg Med Chem, 14, 5584-5591.

26. Ambrus, A., Chen, D., Dai, J., Bialis, T., Jones, R.A. and Yang, D. (2006) Human telomeric sequence forms a hybrid-type intramolecular G-quadruplex structure with mixed parallel/antiparallel strands in potassium solution. Nucleic Acids Res, 34, 2723-2735.

27. Phan, A.T., Luu, K.N. and Patel, D.J. (2006) Different loop arrangements of intramolecular human telomeric (3+1) G-quadruplexes in K+ solution. Nucleic Acids Res, 34, 5715-5719.

28. Luu, K.N., Phan, A.T., Kuryavyi, V., Lacroix, L. and Patel, D.J. (2006) Structure of the human telomere in $\mathrm{K}+$ solution: an intramolecular $(3+1) \mathrm{G}$-quadruplex scaffold. J Am Chem Soc, 128, 99639970.

29. Dai, J., Punchihewa, C., Ambrus, A., Chen, D., Jones, R.A. and Yang, D. (2007) Structure of the intramolecular human telomeric G-quadruplex in potassium solution: a novel adenine triple formation. Nucleic Acids Res, 35, 2440-2450.

30. Matsugami, A., Xu, Y., Noguchi, Y., Sugiyama, H. and Katahira, M. (2007) Structure of a human telomeric DNA sequence stabilized by 8-bromoguanosine substitutions, as determined by NMR in a K+ solution. FEBS J, 274, 3545-3556.

31. Dai, J., Carver, M., Punchihewa, C., Jones, R.A. and Yang, D. (2007) Structure of the Hybrid-2 type intramolecular human telomeric G-quadruplex in $\mathrm{K}+$ solution: insights into structure polymorphism of the human telomeric sequence. Nucleic Acids Res, 35, 4927-4940.

32. Phan, A.T., Kuryavyi, V., Luu, K.N. and Patel, D.J. (2007) Structure of two intramolecular Gquadruplexes formed by natural human telomere sequences in $\mathrm{K}+$ solution. Nucleic Acids Res, 35, 6517-6525.

33. Lim, K.W., Amrane, S., Bouaziz, S., Xu, W.X., Mu, Y.G., Patel, D.J., Luu, K.N. and Phan, A.T. (2009) Structure of the Human Telomere in K+ Solution: A Stable Basket-Type G-Quadruplex with Only Two G-Tetrad Layers. J Am Chem Soc, 131, 4301-4309.

34. Huppert, J.L. and Balasubramanian, S. (2007) G-quadruplexes in promoters throughout the human genome. Nucleic Acids Res, 35, 406-413.

35. Huppert, J.L. (2008) Hunting G-quadruplexes. Biochimie, 90, 1140-1148.

36. Eddy, J. and Maizels, N. (2006) Gene function correlates with potential for G4 DNA formation in the human genome. Nucleic Acids Res, 34, 3887-3896.

37. Balasubramanian, S., Hurley, L.H. and Neidle, S. (2011) Targeting G-quadruplexes in gene promoters: a novel anticancer strategy? Nat Rev Drug Discov, 10, 261-275.

38. Bugaut, A. and Balasubramanian, S. (2012) 5 '-UTR RNA G-quadruplexes: translation regulation and targeting. Nucleic Acids Res, 40, 4727-4741.

39. Huppert, J.L., Bugaut, A., Kumari, S. and Balasubramanian, S. (2008) G-quadruplexes: the beginning and end of UTRs. Nucleic Acids Res, 36, 6260-6268.

40. Arora, A., Dutkiewicz, M., Scaria, V., Hariharan, M., Maiti, S. and Kurreck, J. (2008) Inhibition of translation in living eukaryotic cells by an RNA G-quadruplex motif. Rna, 14, 1290-1296.

41. Beaudoin, J.D. and Perreault, J.P. (2010) 5'-UTR G-quadruplex structures acting as translational repressors. Nucleic Acids Res, 38, 7022-7036.

42. Bonnal, S., Schaeffer, C., Creancier, L., Clamens, S., Moine, H., Prats, A.C. and Vagner, S. (2003) A single internal ribosome entry site containing a $\mathrm{G}$ quartet RNA structure drives fibroblast growth factor 2 gene expression at four alternative translation initiation codons. J Biol Chem, 278, 3933039336.

43. Raymond, E., Sun, D., Chen, S.F., Windle, B. and VonHoff, D.D. (1996) Agents that target telomerase and telomeres. Curr Opin Biotech, 7, 583-591.

44. Zahler, A.M., Williamson, J.R., Cech, T.R. and Prescott, D.M. (1991) Inhibition of telomerase by Gquartet DNA structures. Nature, 350, 718-720.

45. Neidle, S. and Parkinson, G. (2002) Telomere maintenance as a target for anticancer drug discovery. Nat Rev Drug Discov, 1, 383-393.

46. Monchaud, D. and Teulade-Fichou, M.P. (2008) A hitchhiker's guide to G-quadruplex ligands. Org Biomol Chem, 6, 627-636.

47. Maizels, N. and Gray, L.T. (2013) The g4 genome. Plos Genet, 9, e1003468.

48. Randall, A. and Griffith, J.D. (2009) Structure of long telomeric RNA transcripts: the G-rich RNA forms a compact repeating structure containing G-quartets. J Biol Chem, 284, 13980-13986. 
49. Martadinata, H. and Phan, A.T. (2013) Structure of human telomeric RNA (TERRA): stacking of two G-quadruplex blocks in $\mathrm{K}(+)$ solution. Biochemistry, 52, 2176-2183.

50. Schoeftner, S. and Blasco, M.A. (2008) Developmentally regulated transcription of mammalian telomeres by DNA-dependent RNA polymerase II. Nat Cell Biol, 10, 228-236.

51. Cahoon, L.A. and Seifert, H.S. (2009) An Alternative DNA Structure Is Necessary for Pilin Antigenic Variation in Neisseria gonorrhoeae. Science, 325, 764-767.

52. Lopes, J., Piazza, A., Bermejo, R., Kriegsman, B., Colosio, A., Teulade-Fichou, M.P., Foiani, M. and Nicolas, A. (2011) G-quadruplex-induced instability during leading-strand replication. EMBO J, 30, 4033-4046.

53. Piazza, A., Serero, A., Boule, J.B., Legoix-Ne, P., Lopes, J. and Nicolas, A. (2012) Stimulation of gross chromosomal rearrangements by the human CEB1 and CEB25 minisatellites in saccharomyces cerevisiae depends on G-quadruplexes or Cdc13. Plos Genet, 8, e1003033.

54. Davis, J.T. and Spada, G.P. (2007) Supramolecular architectures generated by self-assembly of guanosine derivatives. Chem Soc Rev, 36, 296-313.

55. Williamson, J.R. (1993) Guanine quartets. Curr Opin Struc Biol, 3, 357-362.

56. Hud, N.V., Smith, F.W., Anet, F.A.L. and Feigon, J. (1996) The selectivity for K+ versus Na+ in DNA quadruplexes is dominated by relative free energies of hydration: A thermodynamic analysis by $\mathrm{H}-1$ NMR. Biochemistry, 35, 15383-15390.

57. Gonzalez-Rodriguez, D., van Dongen, J.L.J., Lutz, M., Spek, A.L., Schenning, A.P.H.J. and Meijer, E.W. (2009) G-quadruplex self-assembly regulated by Coulombic interactions. Nat Chem, 1, 151-155.

58. Pinnavaia, T.J., Marshall, C.L., Mettler, C.M., Fisk, C.I., Miles, H.T. and Becker, E.D. (1978) Alkalimetal ion specificity in solution ordering of a nucleotide, 5'-Guanosine monophosphate. $\mathrm{J} \mathrm{Am} \mathrm{Chem}$ Soc, 100, 3625-3627.

59. Cang, X.H., Sponer, J. and Cheatham, T.E. (2011) Explaining the varied glycosidic conformational, G-tract length and sequence preferences for anti-parallel G-quadruplexes. Nucleic Acids Res, 39, 44994512 .

60. Smith, F.W., Lau, F.W. and Feigon, J. (1994) D(G(3)T(4)G(3)) Forms an asymmetric diagonally looped dimeric quadruplex with guanosine 5'-Syn-Syn-Anti and 5'-Syn-Anti-Anti N-glycosidic conformations. P Natl Acad Sci USA, 91, 10546-10550.

61. Webba da Silva, M. (2007) Geometric formalism for DNA quadruplex folding. Chemistry, 13, 97389745 .

62. Phillips, K., Dauter, Z., Murchie, A.I.H., Lilley, D.M.J. and Luisi, B. (1997) The crystal structure of a parallel-stranded guanine tetraplex at 0.95 angstrom resolution. J Mol Biol, 273, 171-182.

63. Hazel, P., Parkinson, G.N. and Neidle, S. (2006) Topology variation and loop structural homology in crystal and simulated structures of a bimolecular DNA quadruplex. J Am Chem Soc, 128, 5480-5487.

64. Parkinson, G.N., Ghosh, R. and Neidle, S. (2007) Structural basis for binding of porphyrin to human telomeres. Biochemistry, 46, 2390-2397.

65. Nielsen, J.T., Arar, K. and Petersen, M. (2009) Solution structure of a locked nucleic acid modified quadruplex: introducing the V4 folding topology. Angew Chem Int Ed Engl, 48, 3099-3103.

66. Neidle, S. (2008) Principles of Nucleic Acid Structure. Elsvier Inc.

67. Phan, A.T. (2010) Human telomeric G-quadruplex: structures of DNA and RNA sequences. FEBS J, 277, 1107-1117.

68. Kerwin, S.M. (2000) G-quadruplex DNA as a target for drug design. Curr Pharm Design, 6, 441-471.

69. Boer, D.R., Canals, A. and Coll, M. (2009) DNA-binding drugs caught in action: the latest 3D pictures of drug-DNA complexes. Dalton T, 3, 399-414.

70. Nanjunda, R., Musetti, C., Kumar, A., Ismail, M.A., Farahat, A.A., Wang, S.M., Sissi, C., Palumbo, M., Boykin, D.W. and Wilson, W.D. (2012) Heterocyclic dications as a new class of telomeric Gquadruplex targeting agents. Curr Pharm Design, 18, 1934-1947.

71. Islam, B., Sgobba, M., Laughton, C., Orozco, M., Sponer, J., Neidle, S. and Haider, S. (2013) Conformational dynamics of the human propeller telomeric DNA quadruplex on a microsecond time scale. Nucleic Acids Res, 41, 2723-2735.

72. Haider, S., Parkinson, G.N. and Neidle, S. (2008) Molecular dynamics and principal components analysis of human telomeric quadruplex multimers. Biophys J, 95, 296-311.

73. Martadinata, H., Heddi, B., Lim, K.W. and Phan, A.T. (2011) Structure of long human telomeric RNA (TERRA): G-quadruplexes formed by four and eight UUAGGG repeats are stable building 
blocks. Biochemistry, 50, 6455-6461.

74. Phan, A.T., Kuryavyi, V., Ma, J.B., Faure, A., Andreola, M.L. and Patel, D.J. (2005) An interlocked dimeric parallel-stranded DNA quadruplex: a potent inhibitor of HIV-1 integrase. P Natl Acad Sci USA, 102, 634-639.

75. Zhang, N., Gorin, A., Majumdar, A., Kettani, A., Chernichenko, N., Skripkin, E. and Patel, D.J. (2001) V-shaped scaffold: a new architectural motif identified in an $A \times(G \times G \times G \times G)$ pentadcontaining dimeric DNA quadruplex involving stacked $\mathrm{G}$ (anti) x $\mathrm{G}$ (anti) x G(anti) x G(syn) tetrads. $J$ Mol Biol, 311, 1063-1079.

76. Tuerk, C. and Gold, L. (1990) Systematic evolution of ligands by exponential enrichment - rna ligands to bacteriophage-T4 DNA-Polymerase. Science, 249, 505-510.

77. Ellington, A.D. and Szostak, J.W. (1990) In vitro selection of RNA molecules that bind specific ligands. Nature, 346, 818-822.

78. Gatto, B., Palumbo, M. and Sissi, C. (2009) Nucleic acid aptamers based on the G-quadruplex structure: therapeutic and diagnostic potential. Curr Med Chem, 16, 1248-1265.

79. Bock, L.C., Griffin, L.C., Latham, J.A., Vermaas, E.H. and Toole, J.J. (1992) Selection of singlestranded-DNA molecules that bind and inhibit human thrombin. Nature, 355, 564-566.

80. Macaya, R.F., Schultze, P., Smith, F.W., Roe, J.A. and Feigon, J. (1993) Thrombin-binding DNA aptamer forms a unimolecular quadruplex structure in solution. P Natl Acad Sci USA, 90, 3745-3749.

81. Padmanabhan, K., Padmanabhan, K.P., Ferrara, J.D., Sadler, J.E. and Tulinsky, A. (1993) The structure of alpha-thrombin inhibited by a 15-mer single-stranded DNA aptamer. J Biol Chem, 268, 17651-17654.

82. Avino, A., Fabrega, C., Tintore, M. and Eritja, R. (2012) Thrombin binding aptamer, more than a simple aptamer: chemically modified derivatives and biomedical applications. Curr Pharm Design, 18, 2036-2047.

83. Do, N.Q., Lim, K.W., Teo, M.H., Heddi, B. and Phan, A.T. (2011) Stacking of G-quadruplexes: NMR structure of a G-rich oligonucleotide with potential anti-HIV and anticancer activity. Nucleic Acids Res, 39, 9448-9457.

84. Jing, N.J., Sha, W., Li, Y.D., Xiong, W.J. and Tweardy, D.J. (2005) Rational drug design of G-quartet DNA as anti-cancer agents. Curr Pharm Design, 11, 2841-2854.

85. Bates, P.J., Laber, D.A., Miller, D.M., Thomas, S.D. and Trent, J.O. (2009) Discovery and development of the G-rich oligonucleotide AS1411 as a novel treatment for cancer. Exp Mol Pathol, 86, 151-164.

86. Dempsey, L.A., Sun, H., Hanakahi, L.A. and Maizels, N. (1999) G4 DNA binding by LR1 and its subunits, nucleolin and hnRNP D, a role for G-G pairing in immunoglobulin switch recombination. $J$ Biol Chem, 274, 1066-1071.

87. Hanakahi, L.A., Sun, H. and Maizels, N. (1999) High affinity interactions of nucleolin with G-Gpaired rDNA. J Biol Chem, 274, 15908-15912.

88. Bates, P.J., Kahlon, J.B., Thomas, S.D., Trent, J.O. and Miller, D.M. (1999) Antiproliferative activity of G-rich oligonucleotides correlates with protein binding. J Biol Chem, 274, 26369-26377.

89. Wyatt, J.R., Vickers, T.A., Roberson, J.L., Buckheit, R.W., Klimkait, T., Debaets, E., Davis, P.W., Rayner, B., Imbach, J.L. and Ecker, D.J. (1994) Combinatorially selected guanosine-quartet structure is a potent inhibitor of human-immunodeficiency-virus envelope-mediated cell-fusion. P Natl Acad Sci USA, 91, 1356-1360.

90. de Soultrait, V.R., Lozach, P.Y., Altmeyer, R., Tarrago-Litvak, L., Litvak, S. and Andreola, M.L. (2002) DNA aptamers derived from HIV-1 RNase $\mathrm{H}$ inhibitors are strong anti-integrase agents. J Mol Biol, 324, 195-203.

91. Marsh, T.C. and Henderson, E. (1994) G-Wires - Self-assembly of a telomeric oligonucleotide, D(GGGGTTGGGG), into large superstructures. Biochemistry, 33, 10718-10724.

92. Marsh, T.C., Vesenka, J. and Henderson, E. (1995) A new DNA nanostructure, the G-wire, imaged by scanning probe microscopy. Nucleic Acids Res, 23, 696-700.

93. Krishnan, Y. and Simmel, F.C. (2011) Nucleic acid based molecular devices. Angew Chem Int Edit, 50, 3124-3156.

94. Lv, L., Guo, Z.J., Wang, J.H. and Wang, E.K. (2012) G-quadruplex as signal transducer for biorecognition events. Curr Pharm Design, 18, 2076-2095.

95. Chinnapen, D.J.F. and Sen, D. (2007) Towards elucidation of the mechanism of UV1C, a 
deoxyribozyme with photolyase activity. J Mol Biol, 365, 1326-1336.

96. Li, T., Wang, E.K. and Dong, S.J. (2008) G-quadruplex-based DNAzyme for facile colorimetric detection of thrombin. Chem Commun, 31, 3654-3656.

97. Li, T., Dong, S.J. and Wang, E. (2009) Label-free colorimetric detection of aqueous mercury ion (Hg2+) using $\mathrm{Hg} 2+-$-modulated G-quadruplex-based DNAzymes. Anal Chem, 81, 2144-2149.

98. Teller, C. and Willner, I. (2010) Functional nucleic acid nanostructures and DNA machines. Curr Opin Biotech, 21, 376-391.

99. Li, Y.F. and Sen, D. (1997) Toward an efficient DNAzyme. Biochemistry, 36, 5589-5599.

100. Travascio, P., Li, Y.F. and Sen, D. (1998) DNA-enhanced peroxidase activity of a DNA aptamer-hemin complex. Chem Biol, 5, 505-517.

101. Nakayama, S. and Sintim, H.O. (2009) Colorimetric split G-quadruplex probes for nucleic acid sensing: Improving reconstituted DNAzyme's catalytic efficiency via probe remodeling. J Am Chem Soc, 131, 10320-10333.

102. Kosman, J. and Juskowiak, B. (2011) Peroxidase-mimicking DNAzymes for biosensing applications: A review. Anal Chim Acta, 707, 7-17.

103. Kawasaki, A.M., Casper, M.D., Freier, S.M., Lesnik, E.A., Zounes, M.C., Cummins, L.L., Gonzalez, C. and Cook, P.D. (1993) Uniformly modified 2'-deoxy-2'-fluoro phosphorothioate oligonucleotides as nuclease-resistant antisense compounds with high affinity and specificity for RNA targets. $J$ Med Chem, 36, 831-841.

104. Wahlestedt, C., Salmi, P., Good, L., Kela, J., Johnsson, T., Hokfelt, T., Broberger, C., Porreca, F., Lai, J., Ren, K.K. et al. (2000) Potent and nontoxic antisense oligonucleotides containing locked nucleic acids. P Natl Acad Sci USA, 97, 5633-5638.

105. Kalota, A., Karabon, L., Swider, C.R., Viazovkina, E., Elzagheid, M., Damha, M.J. and Gewirtz, A.M. (2006) 2'-deoxy-2'-fluoro-beta-D-arabinonucleic acid (2'F-ANA) modified oligonucleotides (ON) effect highly efficient, and persistent, gene silencing. Nucleic Acids Res, 34, 451-461.

106. Peng, C.G. and Damha, M.J. (2007) G-quadruplex induced stabilization by 2'-deoxy-2'-fluoro-Darabinonucleic acids (2'F-ANA). Nucleic Acids Res, 35, 4977-4988.

107. Sagi, J. (2013) G-quadruplexes incorporating modified constituents: a review. J Biomol Struct Dyn.

108. Demple, B., Jacobsson, A., Olsson, M., Robins, P. and Lindahl, T. (1982) Repair of Alkylated DNA in Escherichia-Coli - Physical-Properties of O-6-Methylguanine-DNA Methyltransferase. J Biol Chem, 257, 13776-13780.

109. Gannett, P.M. and Sura, T.P. (1993) Base-Pairing of 8-Oxoguanosine and 8-Oxo-2'-Deoxyguanosine with 2'-Deoxyadenosine, 2'-Deoxycytosine, 2'-Deoxyguanosine, and Thymidine. Chem Res Toxicol, 6, 690-700.

110. Bernstein, B.E., Meissner, A. and Lander, E.S. (2007) The mammalian epigenome. Cell, 128, 669-681.

111. Gros, J., Rosu, F., Amrane, S., De Cian, A., Gabelica, V., Lacroix, L. and Mergny, J.L. (2007) Guanines are a quartet's best friend: impact of base substitutions on the kinetics and stability of tetramolecular quadruplexes. Nucleic Acids Res, 35, 3064-3075.

112. Mekmaysy, C.S., Petraccone, L., Garbett, N.C., Ragazzon, P.A., Gray, R., Trent, J.O. and Chaires, J.B. (2008) Effect of O6-methylguanine on the stability of G-quadruplex DNA. J Am Chem Soc, 130, 6710-6711.

113. Dias, E., Battiste, J.L. and Williamson, J.R. (1994) Chemical probe for glycosidic conformation in Telomeric DNAs. J Am Chem Soc, 116, 4479-4480.

114. Esposito, V., Randazzo, A., Piccialli, G., Petraccone, L., Giancola, C. and Mayol, L. (2004) Effects of an 8-bromodeoxyguanosine incorporation on the parallel quadruplex structure [d(TGGGT)]4. Org Biomol Chem, 2, 313-318.

115. Virgilio, A., Esposito, V., Randazzo, A., Mayol, L. and Galeone, A. (2005) 8-Methyl-2'deoxyguanosine incorporation into parallel DNA quadruplex structures. Nucleic Acids Res, 33, 61886195.

116. Gros, J., Avino, A., de la Osa, J.L., Gonzalez, C., Lacroix, L., Perez, A., Orozco, M., Eritja, R. and Mergny, J.L. (2008) 8-amino guanine accelerates tetramolecular G-quadruplex formation. Chem Commun, 25, 2926-2928.

117. Tran, P.L., Virgilio, A., Esposito, V., Citarella, G., Mergny, J.L. and Galeone, A. (2011) Effects of 8methylguanine on structure, stability and kinetics of formation of tetramolecular quadruplexes. Biochimie, 93, 399-408. 
118. Xu, Y. and Sugiyama, H. (2006) Formation of the G-quadruplex and i-motif structures in retinoblastoma susceptibility genes (Rb). Nucleic Acids Res, 34, 949-954.

119. Benz, A. and Hartig, J.S. (2008) Redesigned tetrads with altered hydrogen bonding patterns enable programming of quadruplex topologies. Chem Commun, 34, 4010-4012.

120. Phan, A.T., Kuryavyi, V., Gaw, H.Y. and Patel, D.J. (2005) Small-molecule interaction with a fiveguanine-tract G-quadruplex structure from the human MYC promoter. Nat Chem Biol, 1, 167-173.

121. Lim, K.W., Lacroix, L., Yue, D.J.E., Lim, J.K.C., Lim, J.M.W. and Phan, A.T. (2010) Coexistence of two distinct G-quadruplex conformations in the hTERT promoter. J Am Chem Soc, 132, 1233112342.

122. He, G.X., Krawczyk, S.H., Swaminathan, S., Shea, R.G., Dougherty, J.P., Terhorst, T., Law, V.S., Griffin, L.C., Coutre, S. and Bischofberger, N. (1998) N-2- and C-8-substituted oligodeoxynucleotides with enhanced thrombin inhibitory activity in vitro and in vivo. J Med Chmy, 41, 2234-2242.

123. Koizumi, M., Akahori, K., Ohmine, T., Tsutsumi, S., Sone, J., Kosaka, T., Kaneko, M., Kimura, S. and Shimada, K. (2000) Biologically active oligodeoxyribonucleotides. Part 12: N2-methylation of 2'deoxyguanosines enhances stability of parallel G-quadruplex and anti-HIV-1 activity. Bioorg Med Chem Lett, 10, 2213-2216.

124. Petersen, M. and Wengel, J. (2003) LNA: a versatile tool for therapeutics and genomics. Trends Biotechnol, 21, 74-81.

125. Pradhan, D., Hansen, L.H., Vester, B. and Petersen, M. (2011) Selection of G-quadruplex folding topology with LNA-modified human telomeric sequences in K+ Solution. Chem-Eur J, 17, 2405-2413.

126. Randazzo, A., Esposito, V., Ohlenschlager, O., Ramachandran, R. and Mayol, L. (2004) NMR solution structure of a parallel LNA quadruplex. Nucleic Acids Res, 32, 3083-3092.

127. Nielsen, J.T., Arar, K. and Petersen, M. (2006) NMR solution structures of LNA (locked nucleic acid) modified quadruplexes. Nucleic Acids Res, 34, 2006-2014.

128. Pasternak, A., Hernandez, F.J., Rasmussen, L.M., Vester, B. and Wengel, J. (2011) Improved thrombin binding aptamer by incorporation of a single unlocked nucleic acid monomer. Nucleic Acids Res, 39, 1155-1164.

129. Tang, C.F. and Shafer, R.H. (2006) Engineering the quadruplex fold: Nucleoside conformation determines both folding topology and molecularity in guanine quadruplexes. J Am Chem Soc, 128, 5966-5973.

130. Sacca, B., Lacroix, L. and Mergny, J.L. (2005) The effect of chemical modifications on the thermal stability of different G-quadruplex-forming oligonucleotides. Nucleic Acids Res, 33, 1182-1192.

131. Zaitseva, M., Kaluzhny, D., Shchyolkina, A., Borisova, O., Smirnov, I. and Pozmogova, G. (2010) Conformation and thermostability of oligonucleotide d(GGTTGGTGTGGTTGG) containing thiophosphoryl internucleotide bonds at different positions. Biophys Chem, 146, 1-6.

132. Datta, B., Schmitt, C. and Armitage, B.A. (2003) Formation of a PNA(2)-DNA(2) hybrid quadruplex. J Am Chem Soc, 125, 4111-4118.

133. Datta, B., Bier, M.E., Roy, S. and Armitage, B.A. (2005) Quadruplex formation by a guanine-rich PNA oligomer. J Am Chem Soc, 127, 4199-4207.

134. Krishnan-Ghosh, Y., Stephens, E. and Balasubramanian, S. (2004) A PNA(4) quadruplex. J Am Chem Soc, 126, 5944-5945.

135. Xu, Y., Suzuki, Y. and Komiyama, M. (2009) Click chemistry for the identification of G-quadruplex structures: discovery of a DNA-RNA G-quadruplex. Angew Chem Int Edit, 48, 3281-3284.

136. Sponer, J., Gabb, H.A., Leszczynski, J. and Hobza, P. (1997) Base-base and deoxyribose-base stacking interactions in B-DNA and Z-DNA: A quantum-chemical study. Biophys J, 73, 76-87.

137. Hobza, P. and Sponer, J. (2002) Toward true DNA base-stacking energies: MP2, CCSD(T), and complete basis set calculations. J Am Chem Soc, 124, 11802-11808.

138. Dabkowska, I., Gonzalez, H.V., Jurecka, P. and Hobza, P. (2005) Stabilization energies of the hydrogen-bonded and stacked structures of nucleic acid base pairs in the crystal geometries of CG, AT, and AC DNA steps and in the NMR geometry of the 5 '-d(GCGAAGC)-3 ' hairpin: Complete basis set calculations at the MP2 and CCSD(T) levels. J Phys Chem A, 109, 1131-1136.

139. Sponer, J., Jurecka, P., Marchan, I., Luque, F.J., Orozco, M. and Hobza, P. (2006) Nature of base stacking: Reference quantum-chemical stacking energies in ten unique B-DNA base-pair steps. ChemEur J, 12, 2854-2865. 
140. Sponer, J., Riley, K.E. and Hobza, P. (2008) Nature and magnitude of aromatic stacking of nucleic acid bases. Phys Chem Chem Phys, 10, 2595-2610.

141. Svozil, D., Hobza, P. and Sponer, J. (2010) Comparison of intrinsic stacking energies of ten unique dinucleotide steps in A-RNA and B-DNA duplexes. Can we determine correct order of stability by quantum-chemical calculations? J Phys Chem B, 114, 1191-1203.

142. Gu, J.D. and Leszczynski, J. (2000) A remarkable alteration in the bonding pattern: An HF and DFT study of the interactions between the metal cations and the Hoogsteen hydrogen-bonded G-tetrad. $J$ Phys Chem A, 104, 6308-6313.

143. Gu, J.D. and Leszczynski, J. (2002) Origin of $\mathrm{Na}+\mathrm{K}+$ selectivity of the guanine tetraplexes in water: The theoretical rationale. J Phys Chem A, 106, 529-532.

144. Meyer, M., Hocquet, A. and Suhnel, J. (2005) Interaction of sodium and potassium ions with sandwiched cytosine-, guanine-, thymine-, and uracil-base tetrads. J Comput Chem, 26, 352-364.

145. Deng, J.P., Xiong, Y. and Sundaralingam, M. (2001) X-ray analysis of an RNA tetraplex (UGGGGU)(4) with divalent Sr2+ ions at subatomic resolution (0.61 angstrom). P Natl Acad Sci USA, 98, 13665-13670.

146. Caceres, C., Wright, G., Gouyette, C., Parkinson, G. and Subirana, J.A. (2004) A thymine tetrad in d(TGGGGT) quadruplexes stabilized with Tl+/Na+ ions. Nucleic Acids Res, 32, 1097-1102.

147. Kondo, J., Adachi, W., Umeda, S., Sunami, T. and Takenaka, A. (2004) Crystal structures of a DNA octaplex with I-motif of G-quartets and its splitting into two quadruplexes suggest a folding mechanism of eight tandem repeats. Nucleic Acids Res, 32, 2541-2549.

148. Lee, M.P.H., Parkinson, G.N., Hazel, P. and Neidle, S. (2007) Observation of the coexistence of sodium and calcium ions in a DNA G-quadruplex ion channel. J Am Chem Soc, 129, 10106-10107.

149. Creze, C., Rinaldi, B., Haser, R., Bouvet, P. and Gouet, P. (2007) Structure of a d(TGGGGT) quadruplex crystallized in the presence of Li+ ions. Acta Crystallogr D Biol Crystallogr, 63, 682-688.

150. Parkinson, G.N., Cuenca, F. and Neidle, S. (2008) Topology conservation and loop flexibility in quadruplex-drug recognition: Crystal structures of inter- and intramolecular telomeric DNA quadruplex-drug complexes. J Mol Biol, 381, 1145-1156.

151. Matsugami, A., Ouhashi, K., Kanagawa, M., Liu, H., Kanagawa, S., Uesugi, S. and Katahira, M. (2001) An intramolecular quadruplex of (GGA)(4) triplet repeat DNA with a G:G:G:G tetrad and a $\mathrm{G}(: \mathrm{A}): \mathrm{G}(\mathrm{A}): \mathrm{G}(\mathrm{A}): \mathrm{G}$ heptad, and its dimeric interaction. J Mol Biol, 313, 255-269.

152. Liu, H., Matsugami, A., Katahira, M. and Uesugi, S. (2002) A dimeric RNA quadruplex architecture comprised of two G:G(:A):G:G(:A) hexads, G:G:G:G tetrads and UUUU loops. J Mol Biol, 322, 955970 .

153. Matsugami, A., Okuizumi, T., Uesugi, S. and Katahira, M. (2003) Intramolecular higher order packing of parallel quadruplexes comprising a $G: G: G: G$ tetrad and a $G(: A): G(: A): G(: A): G$ heptad of GGA triplet repeat DNA. J Biol Chem, 278, 28147-28153.

154. Mashima, T., Matsugami, A., Nishikawa, F., Nishikawa, S. and Katahira, M. (2009) Unique quadruplex structure and interaction of an RNA aptamer against bovine prion protein. Nucleic Acids Res, 37, 6249-6258.

155. Martadinata, H. and Phan, A.T. (2009) Structure of propeller-type parallel-stranded RNA Gquadruplexes, formed by human telomeric RNA sequences in $\mathrm{K}+$ solution. J Am Chem Soc, 131, 2570-2578.

156. Collie, G.W., Haider, S.M., Neidle, S. and Parkinson, G.N. (2010) A crystallographic and modelling study of a human telomeric RNA (TERRA) quadruplex. Nucleic Acids Res, 38, 5569-5580.

157. Mukundan, V.T., Do, N.Q. and Phan, A.T. (2011) HIV-1 integrase inhibitor T30177 forms a stacked dimeric G-quadruplex structure containing bulges. Nucleic Acids Res, 39, 8984-8991.

158. Sponer, J. and Spackova, N. (2007) Molecular dynamics simulations and their application to fourstranded DNA. Methods, 43, 278-290.

159. Cang, X.H., Sponer, J. and Cheatham, T.E. (2011) Insight into G-DNA structural polymorphism and folding from sequence and loop connectivity through free energy analysis. J Am Chem Soc, 133, 14270-14279.

160. Lavery, R., Zakrzewska, K., Sun, J.S. and Harvey, S.C. (1992) A comprehensive classification of nucleic-Acid structural families based on strand direction and base-pairing. Nucleic Acids Res, 20, 5011-5016.

161. Boys, S.F. and Bernardi, F. (1970) Calculation of small molecular interactions by differences of 
separate total energies - Someprocedures with reduced errors. Mol Phys, 19, 553-566.

162. Campbell, N.H., Parkinson, G.N., Reszka, A.P. and Neidle, S. (2008) Structural basis of DNA quadruplex recognition by an acridine drug. J Am Chem Soc, 130, 6722-6724.

163. Collie, G.W., Sparapani, S., Parkinson, G.N. and Neidle, S. (2011) Structural basis of telomeric RNA quadruplex-acridine ligand recognition. J Am Chem Soc, 133, 2721-2728.

164. Clark, G.R., Pytel, P.D., Squire, C.J. and Neidle, S. (2003) Structure of the first parallel DNA quadruplex-drug complex. J Am Chem Soc, 125, 4066-4067.

165. Pan, B.C., Xiong, Y., Shi, K. and Sundaralingam, M. (2003) Crystal structure of a bulged RNA tetraplex at 1.1 angstrom resolution: Implications for a novel binding site in RNA tetraplex. Structure, 11, 1423-1430.

166. Pan, B.C., Xiong, Y., Shi, K., Deng, J.P. and Sundaralingam, M. (2003) Crystal structure of an RNA purine-rich tetraplex containing adenine tetrads: Implications for specific binding in RNA tetraplexes. Structure, 11, 815-823.

167. Pan, B.C., Xiong, Y., Shi, K. and Sundaralingam, M. (2003) An eight-stranded helical fragment in RNA crystal structure: Implications for tetraplex interaction. Structure, 11, 825-831.

168. Pan, B.C., Shi, K. and Sundaralingam, M. (2006) Crystal structure of an RNA quadruplex containing inosine tetrad: Implications for the roles of NH2 group in purine tetrads. J Mol Biol, 363, 451-459.

169. Horvath, M.P. and Schultz, S.C. (2001) DNA G-quartets in a 1.86 A resolution structure of an Oxytricha nova telomeric protein-DNA complex. J Mol Biol, 310, 367-377.

170. Haider, S., Parkinson, G.N. and Neidle, S. (2002) Crystal structure of the potassium form of an Oxytricha nova G-quadruplex. J Mol Biol, 320, 189-200.

171. Haider, S.M., Parkinson, G.N. and Neidle, S. (2003) Structure of a G-quadruplex-ligand complex. J Mol Biol, 326, 117-125.

172. Gill, M.L., Strobel, S.A. and Loria, J.P. (2006) Crystallization and characterization of the thallium form of the Oxytricha nova G-quadruplex. Nucleic Acids Res, 34, 4506-4514.

173. Campbell, N.H., Patel, M., Tofa, A.B., Ghosh, R., Parkinson, G.N. and Neidle, S. (2009) Selectivity in ligand recognition of G-quadruplex loops. Biochemistry, 48, 1675-1680.

174. Campbell, N.H., Smith, D.L., Reszka, A.P., Neidle, S. and O'Hagan, D. (2011) Fluorine in medicinal chemistry: beta-fluorination of peripheral pyrrolidines attached to acridine ligands affects their interactions with G-quadruplex DNA. Org Biomol Chem, 9, 1328-1331.

175. Pan, B., Shi, K. and Sundaralingam, M. (2006) Base-tetrad swapping results in dimerization of RNA quadruplexes: Implications for formation of the i-motif RNA octaplex. P Natl Acad Sci USA, 103, 3130-3134.

176. Wang, Y. and Patel, D.J. (1994) Solution structure of the Tetrahymena telomeric repeat d(T2G4)4 Gtetraplex. Structure, 2, 1141-1156.

177. Balkwill, G.D., Garner, T.P., Williams, H.E. and Searle, M.S. (2009) Folding topology of a bimolecular DNA quadruplex containing a stable mini-hairpin motif within the diagonal loop. J Mol Biol, 385, 1600-1615.

178. Lim, K.W., Lacroix, L., Yue, D.J., Lim, J.K., Lim, J.M. and Phan, A.T. (2010) Coexistence of two distinct G-quadruplex conformations in the hTERT promoter. J Am Chem Soc, 132, 12331-12342.

179. Cornell, W.D., Cieplak, P., Bayly, C.I., Gould, I.R., Merz, K.M., Ferguson, D.M., Spellmeyer, D.C., Fox, T., Caldwell, J.W. and Kollman, P.A. (1995) A second generation force field for the simulation of proteins, nucleic acids, and organic molecules. J Am Chem Soc, 117, 3181-3198.

180. Martadinata, H., Heddi, B., Lim, K.W. and Phan, A.T. (2011) Structure of long human telomeric RNA (TERRA): G-quadruplexes formed by four and eight UUAGGG repeats are stable building blocks. Biochemistry, 50, 6455-6461.

181. Kato, Y., Ohyama, T., Mita, H. and Yamamoto, Y. (2005) Dynamics and thermodynamics of dimerization of parallel G-quadruplexed DNA formed from d(TTAGn) ( $\mathrm{n}=3-5)$. J Am Chem Soc, 127, 9980-9981.

182. Do, N.Q. and Phan, A.T. (2012) Monomer-dimer equilibrium for the 5'-5' stacking of propeller-type parallel-stranded G-quadruplexes: NMR structural study. Chemistry, 18, 14752-14759.

183. Yu, H.Q., Miyoshi, D. and Sugimoto, N. (2006) Characterization of structure and stability of long telomeric DNA G-quadruplexes. J Am Chem Soc, 128, 15461-15468.

184. Xu, Y., Ishizuka, T., Kurabayashi, K. and Komiyama, M. (2009) Consecutive formation of Gquadruplexes in human telomeric-overhang DNA: a protective capping structure for telomere ends. 
Angew Chem Int Ed Engl, 48, 7833-7836.

185. Collie, G.W., Parkinson, G.N., Neidle, S., Rosu, F., De Pauw, E. and Gabelica, V. (2010) Electrospray mass spectrometry of telomeric RNA (TERRA) reveals the formation of stable multimeric Gquadruplex structures. J Am Chem Soc, 132, 9328-9334.

186. Merino, E.J., Boal, A.K. and Barton, J.K. (2008) Biological contexts for DNA charge transport chemistry. Curr Opin Chem Biol, 12, 229-237.

187. Schuster, G.B. (2004) Long-Range Charge Transfer in DNA, I and II; Topics in Current Chemistry. Springer.

188. Genereux, J.C. and Barton, J.K. (2010) Mechanisms for DNA charge transport. Chem Rev, 110, 1642-1662.

189. Venkatramani, R., Keinan, S., Balaeff, A. and Beratan, D.N. (2011) Nucleic acid charge transfer: Black, white and gray. Coordin Chem Rev, 255, 635-648.

190. Siriwong, K. and Voityuk, A.A. (2012) Electron transfer in DNA. Wires Comput Mol Sci, 2, 780-794.

191. Voityuk, A.A., Siriwong, K. and Rosch, N. (2001) Charge transfer in DNA. Sensitivity of electronic couplings to conformational changes. Phys Chem Chem Phys, 3, 5421-5425.

192. Troisi, A. and Orlandi, G. (2002) Hole migration in DNA: a theoretical analysis of the role of structural fluctuations. J Phys Chem B, 106, 2093-2101.

193. von Feilitzsch, T., Tuma, J., Neubauer, H., Verdier, L., Haselsberger, R., Feick, R., Gurzadyan, G., Voityuk, A.A., Griesinger, C. and Michel-Beyerle, M.E. (2008) Chromophore/DNA interactions: Femto- to nanosecond spectroscopy, NMR structure, and electron transfer theory. J Phys Chem B, 112, 973-989.

194. Grozema, F.C., Tonzani, S., Berlin, Y.A., Schatz, G.C., Siebbeles, L.D.A. and Ratner, M.A. (2008) Effect of structural dynamics on charge transfer in DNA hairpins. J Am Chem Soc, 130, 5157-5166.

195. Hatcher, E., Balaeff, A., Keinan, S., Venkatramani, R. and Beratan, D.N. (2008) PNA versus DNA: Effects of structural fluctuations on electronic structure and hole-transport mechanisms. J Am Chem Soc, 130, 11752-11761.

196. Berlin, Y.A., Voityuk, A.A. and Ratner, M.A. (2012) DNA base pair stacks with high electric conductance: A systematic structural search. ACS Nano, 6, 8216-8225.

197. Kotlyar, A.B., Borovok, N., Molotsky, T., Cohen, H., Shapir, E. and Porath, D. (2005) Long, monomolecular guanine-based nanowires. Adv Mater, 17, 1901-1905.

198. Borovok, N., Iram, N., Zikich, D., Ghabboun, J., Livshits, G.I., Porath, D. and Kotlyar, A.B. (2008) Assembling of G-strands into novel tetra-molecular parallel G4-DNA nanostructures using avidinbiotin recognition. Nucleic Acids Res, 36, 5050-5060.

199. Shapir, E., Sagiv, L., Molotsky, T., Kotlyar, A.B., Di Felice, R. and Porath, D. (2010) Electronic structure of G4-DNA by scanning tunneling spectroscopy. J Phys Chem C, 114, 22079-22084.

200. Davis, J.T. (2004) G-quartets 40 years later: From 5 '-GMP to molecular biology and supramolecular chemistry. Angew Chem Int Edit, 43, 668-698.

201. Choi, J., Park, J., Tanaka, A., Park, M.J., Jang, Y.J., Fujitsuka, M., Kim, S.K. and Majima, T. (2013) Hole trapping of G-quartets in a G-quadruplex. Angew Chem Int Edit, 52, 1134-1138.

202. Delaney, S. and Barton, J.K. (2003) Charge transport in DNA duplex/quadruplex conjugates. Biochemistry, 42, 14159-14165.

203. de Champdore, M., De Napoli, L., Montesarchio, D., Piccialli, G., Caminal, C., Mulazzani, Q.G., Navacchia, M.L. and Chatgilialoglu, C. (2004) Excess electron transfer in G-quadruplex. Chem Commun, 15, 1756-1757.

204. Ndlebe, T. and Schuster, G.B. (2006) Long-distance radical cation transport in DNA: horizontal charge hopping in a dimeric quadruplex. Org Biomol Chem, 4, 4015-4021.

205. Huang, Y.C., Cheng, A.K.H., Yu, H.Z. and Sen, D. (2009) Charge conduction properties of a parallel-stranded DNA G-quadruplex: Implications for chromosomal oxidative damage. Biochemistry, 48, 6794-6804.

206. Cohen, H., Sapir, T., Borovok, N., Molotsky, T., Di Felice, R., Kotlyar, A.B. and Porath, D. (2007) Polarizability of G4-DNA observed by electrostatic force microscopy measurements. Nano Lett, 7 , 981-986.

207. Šponer, J. and Lankas, F. (2006) Computational studies of RNA and DNA. Springer, Dordrecht.

208. Cavallari, M., Calzolari, A., Garbesi, A. and Di Felice, R. (2006) Stability and migration of metal ions in G4-wires by molecular dynamics simulations. J Phys Chem B, 110, 26337-26348. 
209. Calzolari, A., Di Felice, R., Molinari, E. and Garbesi, A. (2004) Electron channels in biomolecular nanowires. J Phys Chem B, 108, 2509-2515.

210. Di Felice, R., Calzolari, A., Garbesi, A., Alexandre, S.S. and Soler, J.M. (2005) Strain-dependence of the electronic properties in periodic quadruple helical G4-wires. J Phys Chem B, 109, 22301-22307.

211. Woiczikowski, P.B., Kubar, T., Gutierrez, R., Cuniberti, G. and Elstner, M. (2010) Structural stability versus conformational sampling in biomolecular systems: Why is the charge transfer efficiency in G4DNA better than in double-stranded DNA? J Chem Phys, 133, 035103.

212. Karsisiotis, A.I., Hessari, N.M., Novellino, E., Spada, G.P., Randazzo, A. and da Silva, M.W. (2011) Topological characterization of nucleic acid G-quadruplexes by UV absorption and circular dichroism. Angew Chem Int Edit, 50, 10645-10648.

213. Voityuk, A.A. and Rosch, N. (2002) Fragment charge difference method for estimating donoracceptor electronic coupling: Application to DNA pi-stacks. J Chem Phys, 117, 5607-5616.

214. Voityuk, A.A. (2012) Electronic coupling for charge transfer in donor-bridge-acceptor systems. Performance of the two-state FCD model. Phys Chem Chem Phys, 14, 13789-13793.

215. Ridley, J. and Zerner, M. (1973) Intermediate Neglect of Differential Overlap Technique for Spectroscopy - Pyrrole and Azines. Theor Chim Acta, 32, 111-134.

216. Voityuk, A.A. (2006) Assessment of semiempirical methods for the computation of charge transfer in DNA pi-stacks. Chem Phys Lett, 427, 177-180.

217. Marcus, R.A. and Sutin, N. (1985) Electron Transfers in Chemistry and Biology. Biochimica Et Biophysica Acta, 811, 265-322.

218. Davis, W.B., Hess, S., Naydenova, I., Haselsberger, R., Ogrodnik, A., Newton, M.D. and MichelBeyerle, M.E. (2002) Distance-dependent activation energies for hole injection from protonated 9amino-6-chloro-2-methoxyacridine into duplex DNA. J Am Chem Soc, 124, 2422-2423.

219. Siriwong, K., Voityuk, A.A., Newton, M.D. and Rosch, N. (2003) Estimate of the reorganization energy for charge transfer in DNA. J Phys Chem B, 107, 2595-2601.

220. LeBard, D.N., Lilichenko, M., Matyushov, D.V., Berlin, Y.A. and Ratner, M.A. (2003) Solvent reorganization energy of charge transfer in DNA hairpins. J Phys Chem B, 107, 14509-14520.

221. Kubar, T. and Elstner, M. (2009) Solvent reorganization energy of hole transfer in DNA. J Phys Chem $B, 113,5653-5656$.

222. Haaima, G., Hansen, H.F., Christensen, L., Dahl, O. and Nielsen, P.E. (1997) Increased DNA binding and sequence discrimination of PNA oligomers containing 2,6-diaminopurine. Nucleic Acids Res, 25, 4639-4643.

223. Diaz-Mochon, J.J., Bialy, L., Watson, J., Sanchez-Martin, R.M. and Bradley, M. (2005) Synthesis and cellular uptake of cell delivering PNA-peptide conjugates. Chem Commun, 26, 3316-3318.

224. Paul, A., Sengupta, P., Krishnan, Y. and Ladame, S. (2008) Combining G-quadruplex targeting motifs on a single peptide nucleic acid scaffold: A hybrid (3+1) PNA-DNA bimolecular quadruplex. ChemEur J, 14, 8682-8689.

225. Singh, S.K., Nielsen, P., Koshkin, A.A. and Wengel, J. (1998) LNA (locked nucleic acids): synthesis and high-affinity nucleic acid recognition. Chem Commun, 4, 455-456.

226. Sorensen, J.J., Nielsen, J.T. and Petersen, M. (2004) Solution structure of a dsDNA : LNA triplex. Nucleic Acids Res, 32, 6078-6085.

227. Vester, B., Lundberg, L.B., Sorensen, M.D., Babu, B.R., Douthwaite, S. and Wengel, J. (2002) LNAzymes: Incorporation of LNA-type monomers into DNAzymes markedly increases RNA cleavage. J Am Chem Soc, 124, 13682-13683.

228. Randazzo, A., Esposito, V., Ohlenschlager, O., Ramachandran, R., Virgilio, A. and Mayol, L. (2005) Structural studies on LNA quadruplexes. Nucleos Nucleot Nucl, 24, 795-800.

229. Dominick, P.K. and Jarstfer, M.B. (2004) A conformationally constrained nucleotide analogue controls the folding topology of a DNA G-quadruplex. J Am Chem Soc, 126, 5050-5051.

230. Pedersen, E.B., Nielsen, J.T., Nielsen, C. and Filichev, V.V. (2011) Enhanced anti-HIV-1 activity of Gquadruplexes comprising locked nucleic acids and intercalating nucleic acids. Nucleic Acids Res, 39, 2470-2481.

231. Bonifacio, L., Church, F.C. and Jarstfer, M.B. (2008) Effect of locked-nucleic acid on a biologically active G-quadruplex. A structure-activity relationship of the thrombin aptamer. Int J Mol Sci, 9, 422433.

232. Adrian, M., Heddi, B. and Phan, A.T. (2012) NMR spectroscopy of G-quadruplexes. Methods, 57, 11- 
24.

233. Crnugelj, M., Sket, P. and Plavec, J. (2003) Small change in a G-rich sequence, a dramatic change in topology: New dimeric G-quadruplex folding motif with unique loop orientations. J Am Chem Soc, 125, 7866-7871.

234. Vorlickova, M., Kejnovska, I., Sagi, J., Renciuk, D., Bednarova, K., Motlova, J. and Kypr, J. (2012) Circular dichroism and guanine quadruplexes. Methods, 57, 64-75.

235. Avino, A., Mazzini, S., Ferreira, R., Gargallo, R., Marquez, V.E. and Eritja, R. (2012) The effect on quadruplex stability of North-nucleoside derivatives in the loops of the thrombin-binding aptamer. Bioorg Med Chem, 20, 4186-4193.

236. He, G.X., Williams, J.P., Postich, M.J., Swaminathan, S., Shea, R.G., Terhorst, T., Law, V.S., Mao, C.T., Sueoka, C., Coutre, S. et al. (1998) In vitro and in vivo activities of oligodeoxynucleotide-based thrombin inhibitors containing neutral formacetal linkages. J Med Chem, 41, 4224-4231.

237. Mendelboum Raviv, S., Horvath, A., Aradi, J., Bagoly, Z., Fazakas, F., Batta, Z., Muszbek, L. and Harsfalvi, J. (2008) 4-thio-deoxyuridylate-modified thrombin aptamer and its inhibitory effect on fibrin clot formation, platelet aggregation and thrombus growth on subendothelial matrix. J Thromb Haemost, 6, 1764-1771.

238. Bishop, J.S., Guy-Caffey, J.K., Ojwang, J.O., Smith, S.R., Hogan, M.E., Cossum, P.A., Rando, R.F. and Chaudhary, N. (1996) Intramolecular G-quartet motifs confer nuclease resistance to a potent antiHIV oligonucleotide. J Biol Chem, 271, 5698-5703.

239. Koizumi, M., Koga, R., Hotoda, H., Ohmine, T., Furukawa, H., Agatsuma, T., Nishigaki, T., Abe, K., Kosaka, T., Tsutsumi, S. et al. (1998) Biologically active oligodeoxyribonucleotides. Part 11: The least phosphate-modification of quadruplex-forming hexadeoxyribonucleotide TGGGAG, bearing 3-and 5end-modification, with anti-HIV-1 activity. Bioorg Med Chem, 6, 2469-2475.

240. Kuwasaki, T., Hatta, M., Takeuchi, H. and Takaku, H. (2003) Inhibition of human immunodeficiency virus 1 replication in vitro by a self-stabilized oligonucleotide with 2'-O-methyl-guanosine-uridine quadruplex motifs. J Antimicrob Chemother, 51, 813-819.

241. Urata, H., Kumashiro, T., Kawahata, T., Otake, T. and Akagi, M. (2004) Anti-HIV-1 activity and mode of action of mirror image oligodeoxynucleotide analogue of Zintevir. Biochem Bioph Res Co, 313, 55-61.

242. Deleavey, G.F., Watts, J.K., Alain, T., Robert, F., Kalota, A., Aishwarya, V., Pelletier, J., Gewirtz, A.M., Sonenberg, N. and Damha, M.J. (2010) Synergistic effects between analogs of DNA and RNA improve the potency of siRNA-mediated gene silencing. Nucleic Acids Res, 38, 4547-4557.

243. Pallan, P.S., Greene, E.M., Jicman, P.A., Pandey, R.K., Manoharan, M., Rozners, E. and Egli, M. (2011) Unexpected origins of the enhanced pairing affinity of 2'-fluoro-modified RNA. Nucleic Acids Res, 39, 3482-3495.

244. Martin-Pintado, N., Yahyaee-Anzahaee, M., Deleavey, G.F., Portella, G., Orozco, M., Damha, M.J. and Gonzalez, C. (2013) Dramatic effect of furanose c2' substitution on structure and stability: directing the folding of the human telomeric quadruplex with a single fluorine atom. J Am Chem Soc, 135, 5344-5347.

245. Watts, J.K., Martin-Pintado, N., Gomez-Pinto, I., Schwartzentruber, J., Portella, G., Orozco, M., Gonzalez, C. and Damha, M.J. (2010) Differential stability of 2 ' F-ANA.RNA and ANA.RNA hybrid duplexes: roles of structure, pseudohydrogen bonding, hydration, ion uptake and flexibility. Nucleic Acids Res, 38, 2498-2511.

246. Heddi, B. and Phan, A.T. (2011) Structure of human telomeric DNA in crowded solution. J Am Chem Soc, 133, 9824-9833.

247. Phan, A.T., Kuryavyi, V., Gaw, H.Y. and Patel, D.J. (2005) Small-molecule interaction with a fiveguanine-tract G-quadruplex structure from the human MYC promoter. Nat Chem Biol, 1, 167-173.

248. Hu, L.Y., Lim, K.W., Bouaziz, S. and Phan, A.T. (2009) Giardia Telomeric Sequence d(TAGGG)(4) Forms Two Intramolecular G-Quadruplexes in K+ Solution: Effect of Loop Length and Sequence on the Folding Topology. J Am Chem Soc, 131, 16824-16831.

249. Xu, Y., Noguchi, Y. and Sugiyama, H. (2006) The new models of the human telomere d[AGGG(TTAGGG)(3)] in K+ solution. Bioorgan Med Chem, 14, 5584-5591.

250. Dumas, A. and Luedtke, N.W. (2010) Cation-mediated energy transfer in G-quadruplexes revealed by an internal fluorescent probe. J Am Chem Soc, 132, 18004-18007.

251. Grollman, A.P. and Moriya, M. (1993) Mutagenesis by 8-oxoguanine - an enemy within. Trends Genet, 


\section{9, 246-249.}

252. David, S.S., O'Shea, V.L. and Kundu, S. (2007) Base-excision repair of oxidative DNA damage. Nature, 447, 941-950.

253. Tan, X.Z., Suzuki, N., Johnson, F., Grollman, A.P. and Shibutani, S. (1999) Mutagenic properties of the 8-amino-2 '-deoxyguanosine DNA adduct in mammalian cells. Nucleic Acids Res, 27, 2310-2314.

254. Venkatarangan, L., Sivaprasad, A., Johnson, F. and Basu, A.K. (2001) Site-specifically located 8amino-2 '-deoxyguanosine: thermodynamic stability and mutagenic properties in Escherichia coli. Nucleic Acids Res, 29, 1458-1463.

255. Shrivastav, N., Li, D.Y. and Essigmann, J.M. (2010) Chemical biology of mutagenesis and DNA repair: cellular responses to DNA alkylation. Carcinogenesis, 31, 59-70.

256. Luu, K.N., Phan, A.T., Kuryavyi, V., Lacroix, L. and Patel, D.J. (2006) Structure of the human telomere in K+ solution: An intramolecular (3+1) G-quadruplex scaffold. J Am Chem Soc, 128, 99639970.

257. Gray, D.M., Wen, J.D., Gray, C.W., Repges, R., Repges, C., Raabe, G. and Fleischhauer, J. (2008) Measured and calculated CD spectra of G-quartets stacked with the same or opposite polarities. Chirality, 20, 431-440.

258. Mergny, J.L., Phan, A.T. and Lacroix, L. (1998) Following G-quartet formation by UV-spectroscopy. FEBS Letters, 435, 74-78.

259. Phan, A.T. and Patel, D.J. (2002) A site-specific low-enrichment N-15,C-13 isotope-labeling approach to unambiguous NMR spectral assignments in nucleic acids. J Am Chem Soc, 124, 1160-1161.

260. Hehre, W.J., Radom, L., Schleyer, P.V. and Pople, J.A. (1986) Ab Initio Molecular Orbital Theory. Wiley-Interscience.

261. de la Osa, J.P., Gonzalez, C., Gargallo, R., Rueda, M., Cubero, E., Orozco, M., Avino, A. and Eritja, R. (2006) Destabilization of quadruplex DNA by 8-aminoguanine. Chembiochem, 7, 46-48.

262. Kumar, G.A. and McAllister, M.A. (1998) Theoretical investigation of the relationship between proton NMR chemical shift and hydrogen bond strength. J Org Chem, 63, 6968-6972.

263. Barfield, M., Dingley, A.J., Feigon, J. and Grzesiek, S. (2001) A DFT study of the interresidue dependencies of scalar J-coupling and magnetic shielding in the hydrogen-bonding regions of a DNA tripler. J Am Chem Soc, 123, 4014-4022.

264. Dingley, A.J., Peterson, R.D., Grzesiek, S. and Feigon, J. (2005) Characterization of the cation and temperature dependence of DNA quadruplex hydrogen bond properties using high-resolution NMR. $J$ Am Chem Soc, 127, 14466-14472.

265. Koizumi, M., Koga, R., Hotoda, H., Momota, K., Ohmine, T., Furukawa, H., Agatsuma, T., Nishigaki, T., Abe, K., Kosaka, T. et al. (1997) Biologically active oligodeoxyribonucleotides - IX. Synthesis and anti-HIV-1 activity of hexadeoxyribonucleotides, TGGGAG, bearing 3 '- and 5 '-endmodification. Bioorgan Med Chem, 5, 2235-2243.

266. Juskowiak, B. (2006) Analytical potential of the quadruplex DNA-based FRET probes. Anal Chim Acta, 568, 171-180.

267. Liu, X.Y., Kwan, I.C.M., Wang, S.N. and Wu, G. (2006) G-quartet formation from an N-2-modified guanosine derivative. Organic Letters, 8, 3685-3688.

268. Kaucher, M.S. and Davis, J.T. (2006) N2, C8-disubstituted guanosine derivatives can form G-quartets. Tetrahedron Lett, 47, 6381-6384.

269. Martic, S., Liu, X.Y., Wang, S.N. and Wu, G. (2008) Self-assembly of N-2-modified guanosine derivatives: Formation of discrete G-octamers. Chem-Eur J, 14, 1196-1204.

270. Rachwal, P.A. and Fox, K.R. (2007) Quadruplex melting. Methods, 43, 291-301.

271. Dao, N.T., Haselsberger, R., Michel-Beyerle, M.E. and Phan, A.T. (2013) Excimer Formation by Stacking G-Quadruplex Blocks. Chemphyschem, 14, 2667-2671.

272. Moller, C. and Plesset, M.S. (1934) Note on an approximation treatment for many-electron systems. Phys Rev, 46, 0618-0622.

273. Frisch, M.J.T., G. W.; Schlegel, H. B.; Scuseria, G. E.; Robb, M. A.; Cheeseman, J. R.; Montgomery, Jr., J. A.; Vreven, T.; Kudin, K. N.; Burant, J. C.; Millam, J. M.; Iyengar, S. S.; Tomasi, J.; Barone, V.; Mennucci, B.; Cossi, M.; Scalmani, G.; Rega, N.; Petersson, G. A.; Nakatsuji, H.; Hada, M.; Ehara, M.; Toyota, K.; Fukuda, R.; Hasegawa, J.; Ishida, M.; Nakajima, T.; Honda, Y.; Kitao, O.; Nakai, H.; Klene, M.; Li, X.; Knox, J. E.; Hratchian, H. P.; Cross, J. B.; Adamo, C.; Jaramillo, J.; Gomperts, R.; Stratmann, R. E.; Yazyev, O.; Austin, A. J.; Cammi, R.; Pomelli, C.; Ochterski, J. W.; Ayala, P. Y.; 
Morokuma, K.; Voth, G. A.; Salvador, P.; Dannenberg, J. J.; Zakrzewski, V. G.; Dapprich, S.; Daniels, A. D.; Strain, M. C.; Farkas, O.; Malick, D. K.; Rabuck, A. D.; Raghavachari, K.; Foresman, J. B.; Ortiz, J. V.; Cui, Q.; Baboul, A. G.; Clifford, S.; Cioslowski, J.; Stefanov, B. B.; Liu, G.; Liashenko, A.; Piskorz, P.; Komaromi, I.; Martin, R. L.; Fox, D. J.; Keith, T.; Al-Laham, M. A.; Peng, C. Y.; Nanayakkara, A.; Challacombe, M.; Gill, P. M. W.; Johnson, B.; Chen, W.; Wong, M. W.; Gonzalez, C.; and Pople, J. A.;. (2003). Gaussian, Inc., Pittsburgh PA, pp. and references therein.

274. Hobza, P., Schneider, B., Carsky, P. and Zahradnik, R. (1986) The Superposition Error Problem - the (Hf) 2 and (H2o) 2 Complexes at the Scf and Mp2 Levels. Theochem-J Mol Struc, 31, 377-385.

275. Case, D.A., Cheatham, T.E., Darden, T., Gohlke, H., Luo, R., Merz, K.M., Onufriev, A., Simmerling, C., Wang, B. and Woods, R.J. (2005) The Amber biomolecular simulation programs. J Comput Chem, 26, 1668-1688.

276. Perez, A., Marchan, I., Svozil, D., Sponer, J., Cheatham, T.E., Laughton, C.A. and Orozco, M. (2007) Refinenement of the AMBER force field for nucleic acids: Improving the description of alpha/gamma conformers. Biophys J, 92, 3817-3829.

277. Dupradeau, F.Y., Pigache, A., Zaffran, T., Savineau, C., Lelong, R., Grivel, N., Lelong, D., Rosanski, W. and Cieplak, P. (2010) The R.E.D. tools: advances in RESP and ESP charge derivation and force field library building. Phys Chem Chem Phys, 12, 7821-7839.

278. Jorgensen, W.L., Chandrasekhar, J., Madura, J.D., Impey, R.W. and Klein, M.L. (1983) Comparison of simple potential functions for simulating liquid water. J Chem Phys, 79, 926-935.

279. Berendsen, H.J.C., Postma, J.P.M., Vangunsteren, W.F., Dinola, A. and Haak, J.R. (1984) Moleculardynamics with coupling to an external bath. J Chem Phys, 81, 3684-3690.

280. Vangunsteren, W.F. and Berendsen, H.J.C. (1977) Algorithms for macromolecular dynamics and constraint dynamics. Mol Phys, 34, 1311-1327.

281. Darden, T., York, D. and Pedersen, L. (1993) Particle mesh ewald - an N.Log(N) method for ewald sums in large systems. J Chem Phys, 98, 10089-10092.

282. Plateau, P. and Gueron, M. (1982) Exchangeable proton NMR without base-line distortion, Using new strong-pulse sequences. J Am Chem Soc, 104, 7310-7311.

283. Phan, A.T., Gueron, M. and Leroy, J.L. (2001) Investigation of unusual DNA motifs. Method Enzymol, 338, 341-371.

284. Frisch, M.J., Trucks, G.W., Schlegel, H.B., Scuseria, G.E., Robb, M.A., Cheeseman, J.R., Montgomery, J.A., Jr., Vreven, T., Kudin, K.N., Burant, J.C. et al. 2004. Gaussian 03, Rev. e.01. Gaussian, Wallingford, CT.

285. Ditchfield, R. (1974) Self-consistent perturbation-theory of diamagnetism .1. Gauge-invariant LCAO Method for NMR chemical-shifts. Mol Phys, 27, 789-807. 


\section{Appendix I - List of Publications}

Lech, C.J., Phan, A.T. (2014) Ball with hair: Functionalization of extremely stable G-quadruplex nanoparticles through N2-guanine modifications. (in review)

Li, Z.*, Lech, C.J.*, Phan, A.T. (2014) Sugar-modified G-quadruplexes: effects of LNA-, 2'F-RNA- and 2'F-ANA-guanosine chemistries on G-quadruplex structure and stability. Nucleic Acids Research, [in press]. (* Denotes co-first authorship)

Lech, C.J., Phan, A.T., Michel-Beyerle, M., Voityuk, A.A. (2013) Electron-hole transfer in G-quadruplexes with different tetrad stacking geometries: A combined QM and MD study. Journal of Physical Chemistry B, 117, 9851-9856

Lech, C.J., Heddi, B., Phan, A.T. (2013) Guanine base stacking in G-quadruplex Nucleic Acids. Nucleic Acids Research, 41 (3). 2034-2046.

Lech, C.J., ${ }^{*}$ Zhe, Li., ${ }^{*}$ Heddi, B., Phan, A.T. (2012) 2'-F-ANA-guanosine and 2'F-guanosine as powerful tools for structural manipulation of G-quadruplexes. Chemical Communications. 48. 11425-11427. (* Denotes co-first authorship)

Lech, C.J., Lim, J.K.C., Lim, J.M.W., Amrane, S., Heddi, B., Phan, A.T. (2011) Effects of Site-Specific Guanine C8-Modifications on an intramolecular DNA Gquadruplex. Biophysical Journal. 101. 1987-1996. 


\section{Appendix II - Protocols}

\section{Chapter 2: Fundamentals of G-quadruplexes: Base Stacking and High-Order Assemblies}

\section{Model Tetrads}

Tetrad models were created by first performing QM geometry optimization of a single G-tetrad at the MP2/6-31(d,p) level (260,272). Optimized structures consisted of planar G-tetrads displaying C4-symmetry stabilized by two centrally located cations, located above and below the tetrad plane. Optimized tetrads were then used to create models used for single-point energy calculations which contain two parallel centered planar G-tetrads with a single, central $\mathrm{K}^{+}$ion. The geometries of stacked planar tetrads were varied by their separation $\left(d_{t e t}\right)$ and relative rotation $\left(\theta_{\text {tet }}\right)$.

\section{Structural Characterization}

A survey of G-quadruplex structures published in the Protein Data Bank was carried out using the following keywords: quadruplex(es); tetrad(s); tetraplex(es). Search results of X-ray crystallographic structures of G-quadruplexes containing neighboring G-tetrads as of $16^{\text {th }}$ June, 2011 are presented. A selection of NMR G-quadruplex solution structures containing stacked G-tetrads is also presented. The relative rotation $\left(\theta_{t e t}\right)$ and separation $\left(d_{t e t}\right)$ of individual pairs of stacked guanine bases in experimentally determined structures were quantified by comparison with a library of stacked guanines taken from our models of stacked tetrads with geometries varying in rotation and separation with increments of $0.05^{\circ}$ and $0.005 \AA$, respectively. The characterization of guanines within G-quadruplexes involved the alignment of over 1000 experimentally determined stacked guanine pairs (from 40 crystallographic and 16 NMR PDB structures) to a library of model stacked guanines. The pair of model stacked guanines that best fit a given experimental pair was determined by the lowest RMSD (C,N,O atoms) alignment of the guanine bases. A single RMSD minimum was observed in rotation-separation space for every guanine pair cataloged. Stacked guanines within G-tetrads tend to be parallel although Gtetrads are not always planar. Characterization of individual stacked base separations is an accurate measure of inter-base stacking distance and is relevant to our computational study involving planar and parallel G-tetrad stacks. Structural characterization code was written using MATLAB software (version R2008B, The Mathworks Inc.). Structural visualization was performed using PyMOL molecular visualization software (version 0.99, http://www.pymol.org/). 
Experiment

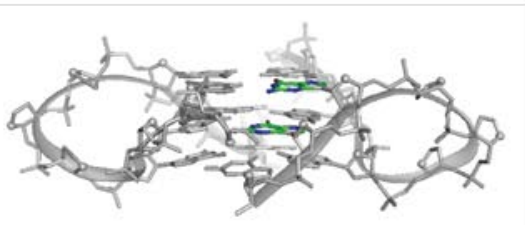

Experimental stacked guanines

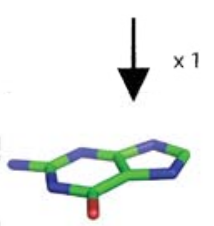

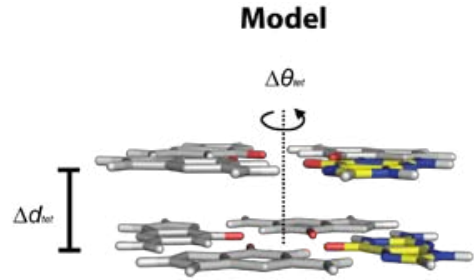

Model stacked guanine

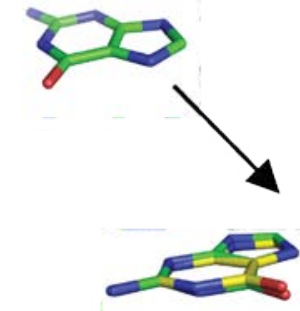

Alignment

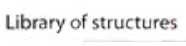<smiles>CCCCCC1CCCCC1</smiles>
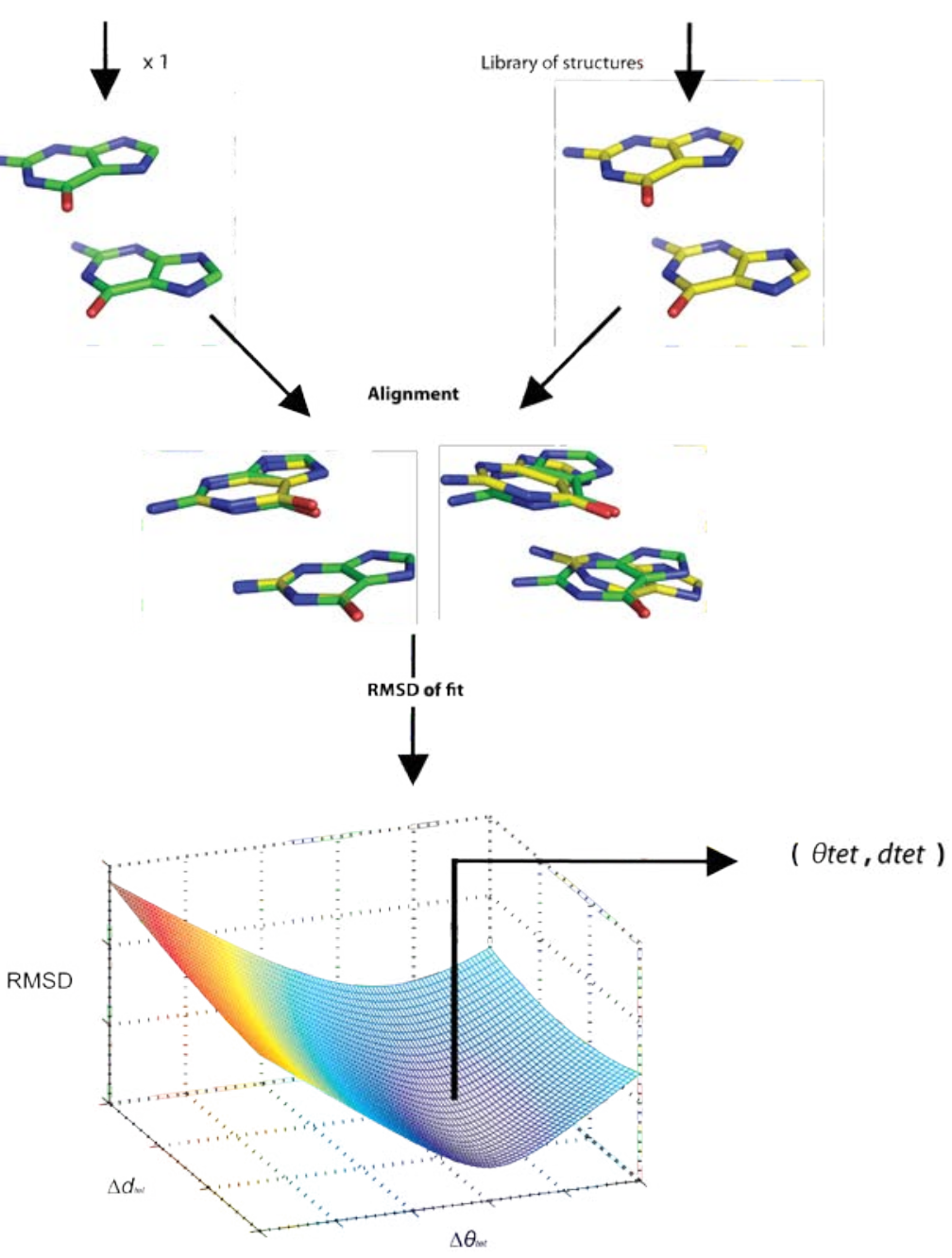

Figure A2-1: Experimental Characterization Procedure: Stacked guanine bases from experimental tetrads are fit to stacked guanines from a library of model stacked guanine tetrads. The relative rotation ( $\theta$ tet) and separation (dtet) are determined by the model stacked guanine pair that provides the lowest RMSD fit to the experimental guanine pair.

\section{Quantum Mechanical Computations}

QM computations were carried out using the Gaussian 03 software (273). Single-point interaction energy calculations used to create energy landscapes were performed at the MP2 level (272) using the modified split valance basis set $6-31 G^{*}(0.25)$ (274) in which the polarization d-function exponent from the standard 6-31G* basis set (260) is changed from 0.8 to 0.25 for first row atoms (Carbon, Nitrogen, Oxygen) as well as for Potassium ions. Previous works by Hobza et al. (137) and Šponer et al. (140) have demonstrated the 6-31G*(0.25) basis set to be an inexpensive choice for modeling stacking interactions. The $6-31 G^{*}(0.25)$ basis set was further tested here. 
Energy landscapes were determined for both same-polarity and opposite-polarity stacked Gtetrads. Single-point interaction energy was computed over a tetrad separation distance $\left(d_{t e t}\right)$ of 3.0-3.6 $\AA$ with a $0.1 \AA$ resolution and a relative rotation angle $\left(\theta_{t e t}\right)$ of $0^{\circ}-90^{\circ}$ with $5^{\circ}$ resolution. In total, 266 single-point interaction energy calculations were performed at the MP2/6-31G*(0.25) level. Single-point energy calculations were also performed for average geometries of base stacking modes observed in crystal structures at the MP2/6-311+G(2d,2p) level $(260,272)$. Interaction energies $(\Delta \mathrm{E})$ in this work have been determined using the counterpoise procedure (161) to correct for basis set superposition error treating each tetrad layer and central ion as individual fragments.

\section{Molecular Mechanics and Dynamic Computations}

MM and MD computations were performed with the AMBER 10 software (275) using the force field of Cornell et al. (179) with the Parmbsc0 force field modification (276) commonly employed for nucleic acid simulations. The intermolecular terms effecting the energy landscape of MM computed stacked G-tetrads include Van der Waals and electrostatic terms as described by the original force field of Cornell et al. (179). Single-point energy calculations used to generate energy landscapes were performed using the same models employed in QM computation. Energy landscape calculations were performed in vacuum with no cutoff used for non-bonded interactions. Force field parameters for isolated guanines used in our models were derived by standard AMBER protocols. RESP partial atomic charges were calculated at HF/631G* level using the R.E.D. software (277).

Molecular dynamic simulations of stacked G-quadruplexes were generated from an NMR based model of the sequence $d\left[T T\left(\mathrm{G}_{3} \mathrm{~T}\right)_{4}\right]$ (182). Flanking ends were removed to generate a model for the $d\left[\left(G_{3} T\right)_{3} G_{3}\right]$ sequence used in our simulations. The chemistry of strand termini were modified to reflect that observed in experimentally synthesized oligonucleotides with the 3'-end capped with a C3'-O3'-H chemistry and the 5'-end capped with a C5'-O5'-H chemistry. Two individual G-quadruplex units were then manually stacked at their 5'-5' and 3'-3' stacking. The relative rotation of these units was varied in $\sim 30^{\circ}$ intervals to generate 3 unique starting structures for each of the 5' -5 ' and 3'-3' stacking models. Starting structures ranged over two of the four possible stacking quadrants. $\mathrm{K}^{+}$ions were manually added between tetrads in the core and at the interface of stacked G-quadruplexes. Additional $\mathrm{K}^{+}$ions (for a total of 28) were added to neutralize the systems. The systems were solvated with a TIP3P (278) water truncated octahedral box with water molecules ranging in number from 5,500-7,500 over the models explored.

Periodic boundary conditions were used in our simulations. Pressure and temperature were held constant at 1 bar and $300 \mathrm{~K}$ respectively according to the Berendsen algorithm (279). A 2-fs time step was used while constraining covalent hydrogen bonds using SHAKE (280). The particle mesh Ewald (PME) method (281) was used to calculate long range electrostatic interactions. Non-bonded interactions were calculated within a $9 \AA$ cutoff in our MD simulations and the list of non-bonded pairs was updated and the center-of-mass motion was removed at $10 \mathrm{ps}$ intervals. Systems underwent a series of initial constrained minimizations and equilibration dynamics. Systems were first minimized with harmonic potential position restraints $\left(25 \mathrm{kcal}_{\mathrm{mol}} \mathrm{l}^{-1} . \AA^{-2}\right)$ on DNA atoms and ions within the G-quadruplex core/interface over 500 steps of steepest decent minimization followed by 500 step of conjugated gradient minimization. Systems were then heated from 100 to $300 \mathrm{~K}$ over $10 \mathrm{ps}$ and equilibrated at $300 \mathrm{~K}$ for $90 \mathrm{ps}$ under constant volume while maintaining $25 \mathrm{kcal} . \mathrm{mol}^{-1} . \AA^{-2}$ position restraints on above mentioned atoms. Systems underwent further steps of minimization and equilibration in

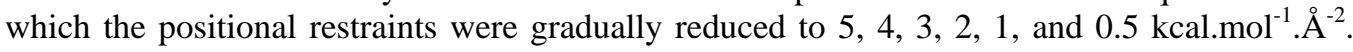
We take the start of simulations as the first segment of unrestrained dynamic simulation. Structures were examined every $25 \mathrm{ps}$ in the first $3 \mathrm{~ns}$ and every $100 \mathrm{ps}$ thereafter. The guanines at the interface were compared to model stacked guanines of variable separation and relative rotation. All four stacked guanine pairs at the interface were cataloged for each snap shot and the average rotational values are presented. 


\title{
Chapter 3: Fundamentals of G-quadruplexes: Charge Transport
}

\author{
Molecular Dynamics simulations
}

Molecular Dynamics simulations (MD) were performed with the AMBER program using the Parmbsc0 correction to the Cornell et al. force field $(179,275,276)$ in explicit solvent in the presence of neutralizing $\mathrm{K}^{+}$ions described previously (see Chapter 2 Methods and Materials) . The G-tetrad stacking modes adopted by a given G-quadruplex are dependent on structural conformation and the ability to form stacked dimers. Considering this, we explore multiple MD systems in order to obtain data on all 5 of the previously observed G-tetrad stacking modes: 1) Complexes of parallel G-quadruplexes stacked in different orientations were examined. Gquadruplex structures formed by the $d\left[\left(G_{3} T\right)_{3} G_{3}\right]$ sequences were derived from an NMR based model (182). Simulations of 200 ns were run for various orientations of stacked G-quadruplexes. 2) Simulations of the $(2+2)$ anti-parallel G-quadruplex structure formed by the sequence $\mathrm{d}\left[\left(\mathrm{G}_{4} \mathrm{~T}_{4} \mathrm{G}_{4}\right)_{2}\right]$ were performed using the same protocol with a simulation time of $5 \mathrm{~ns}$. The starting structure for the simulation of $\mathrm{d}\left[\left(\mathrm{G}_{4} \mathrm{~T}_{4} \mathrm{G}_{4}\right)_{2}\right]$ was taken from a crystal structure ID 1JRN (170).

\section{Quantum Chemical Calculations}

G-tetrad stacks examined at the QM level were taken from 1ns segments of above mentioned MD trajectories. Snapshots were analyzed at 1 ps intervals leading to 1000 unique G-tetrad stacks for each G-tetrad stacking mode. G-tetrad models were created by stripping away backbone/sugar atoms from the G-quadruplex and isolating a single stack G-tetrads including the coordinated $\mathrm{K}^{+}$ion from MD snapshots. The $\mathrm{C1}$ ' atom of the sugar moiety was replaced by a hydrogen atom with the bond length adjusted to $1.08 \AA$. The structures were examined using the INDO/S method (215). As shown previously, the INDO/S scheme allows one to properly describe electronic coupling of stacked nucleobases (216). For each G-tetrad stack, eight electronic states of the lowest energy corresponding to the radical cation were analyzed. These states were represented by 8 highest occupied MOs (HOMOs) of the neutral species $(\mathrm{HOMO} \rightarrow \mathrm{HOMO}-7)$ and are associated with each of the eight guanine bases in the G-tetrad stack.

Several types of control calculations were performed. To test the effects of electrostatic contributions from solvent on coupling matrix elements, models with and without electrostatic embedding were treated. Point charges taken from the Cornell et al. force field were placed at the coordinates of atoms in the fully represented systems consisting of nucleobases and the sugar-phosphate backbone hydrated in water and $\mathrm{K}+$ ions. We also tested the effects of excluding the coordinating $\mathrm{K}^{+}$ion from the stack. It was found that while these changes affected the energies of the hole states, they did not significantly affect the electronic coupling of stacked guanines. 


\section{Chapter 4: Engineering the Sugar Backbone: Locked Nucleic Acid}

\section{DNA sample preparation}

All DNA sequences used in this study were synthesized on a Applied Biosystems 394 DNA

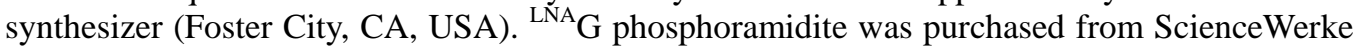
(Singapore). Deuterium-labeled guanine phosphoramidites was purchased from Glen Research (Sterling, VA, USA). After purification, samples were dialyzed successively against de-ionised water, $\mathrm{KCl}$ solution $(25 \mathrm{mM})$ and de-ionised water again. After lyophilization, DNA was dissolved and stored in potassium phosphate (KPi) buffer $\left(\mathrm{pH} 7,10 \% \mathrm{D}_{2} \mathrm{O}\right.$ and $\left.90 \% \mathrm{H}_{2} \mathrm{O}\right)$. The concentrations of KPi were $5 \mathrm{mM}$ for the PS-series series and $20 \mathrm{mM}$ for the HT-series. DNA concentration is expressed in strand molarity with the extinction coefficient of modified sequences approximated to that of the unmodified sequence.

\section{Circular Dichroism spectroscopy}

Circular Dichroism (CD) experiments were performed on JASCO-815 spectropolarimeter (Tokyo, Japan) using 1-cm path length quartz cuvette. CD spectra were taken at $20^{\circ} \mathrm{C}$ in the range of 220-320 nm and accumulated over 3 scans for the PS-series and 10 scans for the HTseries. Spectra were base-line corrected and zero-corrected at $320 \mathrm{~nm}$. CD spectra were normalized by strand concentration determined by concurrent UV absorbance measurements. Samples for the PS-series were prepared by diluting with $1 \mathrm{mM} \mathrm{KPi}$ buffer to obtain DNA concentration of $3 \mu \mathrm{M}$ and KPi concentration of $1.1 \mathrm{mM}$. Samples for the HT-series series were prepared by diluting with $20 \mathrm{mM}$ KPi buffer to a DNA concentration of $10 \mu \mathrm{M}$.

\section{Circular Dichroism melting}

CD melting was performed for PS-series and loop-elongated samples by monitoring CD intensity at $260 \mathrm{~nm}$ against temperature over a range of $45-95^{\circ} \mathrm{C}$. CD melting was performed by stabilizing the sample at $95^{\circ} \mathrm{C}$ for 20 minutes and then cooling at rate of $0.2^{\circ} \mathrm{C} / \mathrm{min}$. Samples were then stabilized at $45^{\circ} \mathrm{C}$, and heated up again to $95^{\circ} \mathrm{C}$ at the same rate. For several samples, this range was extended to a lower temperature. $\mathrm{T}_{\mathrm{m}}$ values were obtained by baseline normalization of CD melting curves and calculation of the faction folded. Presented $T_{m}$ values are an average between cooling and heating curves.

\section{UV Melting}

UV melting experiments were performed on a JASCO V-650 UV-Vis spectrophotometer (Tokyo, Japan). UV melting experiments were done for HT-series samples by monitoring UV absorbance at $295 \mathrm{~nm}$ (258) over a temperature range of $14-83^{\circ} \mathrm{C}$ using a 1 -cm path length quartz cuvette. For several samples, this range was extended to a lower temperature. UV melting was done by first stabilizing at $83^{\circ} \mathrm{C}$ for 30 minutes. Samples were cooled down to $14^{\circ} \mathrm{C}$ at rate of $0.1^{\circ} \mathrm{C} / \mathrm{min}$, stabilized, and heated up to $83^{\circ} \mathrm{C}$ at the same rate. Melting curves were normalized by baseline correction to obtain fraction folded curves (270). $T_{m}$ values presented are an average over cooling and heating curves. Samples for the HT-series were prepared by diluting with $20 \mathrm{mM} \mathrm{KPi}$ to obtain a DNA concentration of $10 \mu \mathrm{M}$.

\section{NMR Spectroscopy}

NMR experiments were performed on 600 or $700 \mathrm{MHz}$ Bruker NMR spectrometer at $25^{\circ} \mathrm{C}$ using a jump and return-type water suppression pulse sequence $(282,283)$. Chemical shift was calibrated by DSS (4,4-dimethyl-4-silapentane-1-sulfonic acid) in NMR buffer. Prior to recording NMR spectra, samples were annealed by heating at $100^{\circ} \mathrm{C}$ for 5 minutes and cooled down slowly overnight. Samples of PS-series contained $0.15 \mathrm{mM}$ DNA in $5 \mathrm{mM}$ KPi buffer, while samples of HT-series contained 0.1-0.2 mM DNA in the $20 \mathrm{mM}$ KPi buffer. For 2D NMR experiments, the DNA concentration was $\sim 1.5 \mathrm{mM}$. 


\section{Structural Calculation}

NMR structural computation was performed by the XPLOR-NIH program (40). Distance restraints, dihedral restraints, hydrogen bond restraints and planarity restraints were used to compute the structure of the HT-L4 sequence. Dihedral angle restraints for $\chi$ were imposed on all guanines except for ${ }^{\mathrm{LNA}} \mathrm{G} 4$. Dihedral angle restraints for $\varepsilon$ were imposed on all G-tetrad dinucleotide steps expect for G3-to- ${ }^{\mathrm{LNA}} \mathrm{G} 4$ and ${ }^{\mathrm{LNA}} \mathrm{G} 4-$ to-G5 steps. Distance restraints were deduced from NOESY experiments at different mixing times. Through the structural refinement and computational procedures described, we present the 10 lowest energy structures from an original ensemble of 100. Structures were visualized using PyMOL software.

\section{Structural Data Deposition}

The coordinates of the 10 lowest energy NMR solution structures of the (3+1) G-quadruplex formed by the $d\left[T_{2} G\left({ }^{L N A} G\right) G_{2} A\left(G_{3} T_{2} A\right)_{2} G_{3} A\right]$ sequence (HT-L4) will be deposited into the Protein Data Bank upon publication. 


\section{Chapter 5: Engineering the Sugar Backbone: 2'-F- Guanosine and 2'-F-ANA-Guanosine Modifications}

\section{DNA sample preparation}

Oligonucleotides were chemically synthesized using an Applied Biosystems 394 DNA/RNA synthesizer (Foster City, CA, U.S.A.). Phosphoramidites of 2'-deoxy-2'-fluoro-guanosine and 2'-deoxy-2'-fluoroarabino-guanosine were purchased from Glen Research (Sterling, VA, U.S.A.). Oligonucleotides were then purified using a Poly-Pak cartridge (Glen Research) following standard protocol. Purified samples were dialyzed successively against water, $\mathrm{KCl}$ solution, and water. Samples were then lyophilized and dissolved in a solution of $5 \mathrm{mM} \mathrm{KCl}$ and $5 \mathrm{mM}$ potassium phosphate $(\mathrm{pH} \mathrm{7)}$ for the HT-series sequences and $1 \mathrm{mM}$ potassium phosphate ( $\mathrm{pH}$ 7) for the PS-series sequences, containing $10 \% \mathrm{D}_{2} \mathrm{O}$ and $20 \mu \mathrm{M}$ 4,4-dimethyl-4silapentane-1-sulfonic acid (DSS).

\section{NMR spectroscopy}

One-dimensional ${ }^{1} \mathrm{H}$ NMR spectra of unmodified and modified sequences were recorded on a 600 or $700 \mathrm{MHz}$ Bruker spectrometer (Billerica, MA. USA) at $25^{\circ} \mathrm{C}$. Samples were heated to $100^{\circ} \mathrm{C}$ for 5 minutes and allowed to slowly cooled overnight prior to NMR and subsequent CD studies. DNA strand concentration was $0.05 \mathrm{mM}$ for the PS-series samples and in the range of 0.05-0.4 mM for the HT-series samples. Spectra were recorded using a jump-and-return-type pulse sequence for water suppression $(282,283)$. Chemical shifts were internally referenced using DSS.

\section{Circular dichroism}

CD spectra were taken using a JASCO-815 spectropolarimeter (Tokyo, Japan). DNA strand concentration was 4-6 $\mu \mathrm{M}$. Spectra were recorded at $20^{\circ} \mathrm{C}$ in a wavelength range of $220-320 \mathrm{~nm}$ and averaged over 10 accumulations. Spectra were zero corrected at $320 \mathrm{~nm}$ and normalized for concentration by concurrent UV absorbance measurements.

\section{UV denaturing experiments}

Thermal denaturation was monitored using a JASCO V-650 UV-visible spectrophotometer (Tokyo, Japan). DNA strand was concentration of 4-6 $\mu \mathrm{M}$. For the PS-series sequences, samples were first cooled from 89 to $42^{\circ} \mathrm{C}$ at a rate of $0.2^{\circ} \mathrm{C} / \mathrm{min}$, then allowed to equilibrate for 10 minutes and heated at the same rate over the same range. The HT-series sequences were treated in a similar manner between the range of $83^{\circ} \mathrm{C}$ and $19^{\circ} \mathrm{C}$. This range was extended for certain sequences in order to monitor the complete transition. The UV absorbance of samples was recorded at $295 \mathrm{~nm}$ and corrected by subtracting the absorbance at $320 \mathrm{~nm}$ as well as baseline corrected with a reference cell. Melting curves were then normalized to create folded fraction curves $(258,270)$. The melting temperature $\left(T_{m}\right)$ is determined to be the temperature at which half of the sample is in the folded state.

\section{CD denaturing experiments}

Thermal denaturation was monitored using CD spectroscopy to confirm UV transitions for some select samples. Experiments were recorded in a similar manner to UV experiments. The CD intensity was recorded at $260 \mathrm{~nm}$ for the PS-series sequences and $295 \mathrm{~nm}$ for the HT-series sequences and corrected by subtracting the intensity at $320 \mathrm{~nm}$. CD melting experiments were normalized in a manner similar to UV melting experiments. 


\section{Chapter 6: Engineering the Base: C8-modified Guanines}

\section{DNA sample preparation}

The unlabeled and the site-specific low-enrichment $\left(2 \%{ }^{15} \mathrm{~N}\right.$-labeled) DNA oligonucleotides were chemically synthesized on an ABI 394 DNA synthesizer and purified as previously described (259). Phosphoramidites of guanine derivatives were purchased from Glen Research. ${ }^{15} \mathrm{~N}$-labeled phosphoramidites were purchased from Cambridge Isotope. Samples were dialyzed successively against $\mathrm{KCl}$ solution and against water.

\section{UV spectroscopy}

Experiments were performed on a Varian 300 Cary Bio UV-Vis spectrophotometer. Samples contained 2-10 $\mu \mathrm{M}$ DNA in a solution of $20 \mathrm{mM}$ potassium phosphate $(\mathrm{pH} 7)$. The thermal stability for each sequence was determined by recording the absorbance at $295 \mathrm{~nm}$ (258). Heating and cooling $\left(15-88^{\circ} \mathrm{C}\right)$ was performed at a rate of $0.5^{\circ} \mathrm{C} / \mathrm{min}$. Quantitative melting temperature $\left(\mathrm{T}_{\mathrm{m}}\right)$ data presented represents an average over an annealing cycle. Hysteresis of less than $1.5^{\circ} \mathrm{C}$ was observed for all samples.

\section{CD spectroscopy}

Experiments were performed on a JASCO-815 spectropolarimeter. Samples contained 2-10 $\mu \mathrm{M}$ DNA in a solution of $20 \mathrm{mM}$ potassium phosphate ( $\mathrm{pH}$ 7). Samples were heated to $90^{\circ} \mathrm{C}$ for 5 minutes and left to cool down to room temperature. CD spectra were monitored at $20^{\circ} \mathrm{C}$ from 220 to $320 \mathrm{~nm}$ and averaged over 10 accumulation cycles. Data were zero-corrected at $320 \mathrm{~nm}$ and finally normalized by strand concentration determined by concurrent UV absorbance measurements at $260 \mathrm{~nm}$.

\section{NMR spectroscopy}

Experiments were performed on 600 and $700 \mathrm{MHz}$ Bruker NMR spectrometers at $25^{\circ} \mathrm{C}$ using a jump-and-return-based water suppression pulse sequence $(282,283)$. DNA concentration was typically $0.2-0.7 \mathrm{mM}$. Samples contained $\mathrm{KCl}(70$ or $150 \mathrm{mM})$ and $20 \mathrm{mM}$ potassium phosphate ( $\mathrm{pH} 7$ ). We also monitored several sequences in a low salt condition (only $20 \mathrm{mM}$ potassium phosphate) and did not observe significant changes in NMR spectra. NMR chemical shifts were referenced with 4,4-dimethyl-4-silapentane-1-sulfonic acid (DSS). Resonances were assigned unambiguously for $\mathrm{G}_{\delta}$ imino protons for most sequences using site-specific lowenrichment labeling (259).

\section{Ab initio computation}

Quantum mechanical calculations were performed using the GAUSSIAN 03 software (284). Molecular geometries were optimized at the Hartree-Fock level using four standard splitvalance basis sets including 6-31G(d), 6-31G(d,p), 6-31++G(d,p), and 6-311++G(d,p) (260). Tight optimization criteria were imposed for optimized geometries. NMR chemical shifts were calculated using the GIAO method (285) at the Hartree-Fock level using the above mentioned standard split-valance basis sets as well as the 6-311+G(d) basis set (260).

Models consisted of four guanines arranged in a planar tetrad with two centrally located potassium ions placed at a distance of $1.7 \AA$ above and below the tetrad plane. Initial guanines were arranged within the $\mathrm{Z}=0$ plane allowing the tetrad to maintain planarity during optimization. The unmodified models underwent initial geometry optimization resulting in a $\mathrm{C}_{4}$ symmetry with identical hydrogen-bond geometry. A single guanine was modified at the C8 position by adding a modification group (Bromo-, Amino-, or O-methyl-) within the plane of the base. In the case of ${ }^{\text {Omet }} \mathrm{G}$, a cis conformation of the $\mathrm{N}_{7}-\mathrm{C}_{8}-\mathrm{O}_{\mathrm{R}}-\mathrm{C}_{\mathrm{R}}$ bond was chosen. This was done to maintain planarity and was thought to be a more likely than the trans conformation, which may crash with sugar-phosphate backbone in the G-quadruplex structure. Geometry 
optimization was again performed after substitution of a single guanine with a C8-modified guanine derivative. NMR chemical shift calculations were carried out on the unmodified and modified models.

A total of 16 individual molecular geometries (Unmodified, Bromo-, Amino-, and O-methylcontaining models each optimized with 4 different basis sets) were calculated. The geometries of modified tetrads were compared to the geometries of the unmodified tetrads as presented in Tables 5 and 6. A total of 80 individual chemical shifts (16 individual geometries evaluated with 5 different basis sets for NMR chemical shift calculations) were computed.. 


\section{Chapter 7: Engineering the Base: N2-modified Guanines}

\section{Synthesis and sample preparation}

DNA samples were synthesized on an Applied Biosystems 394 DNA Synthesizer (Foster City, CA U.S.A.) and purified following Glen Research protocols. The modified phosphoramidite N2-6-amino-hexyl-guanine was purchased from Glen Research (Sterling, VA U.S.A.). Modified phosphoramidites N2-methyl-guanine and N2-benzyl-guanine were purchased from Berry \& Associates (Dexter, MI U.S.A.). Purified DNA samples were dialyzed against $\mathrm{H}_{2} \mathrm{O}, \sim 30 \mathrm{mM}$ $\mathrm{KCl}$, and $\mathrm{H}_{2} \mathrm{O}$ before being dried and dissolved in solution containing $\mathrm{KCl}$, potassium phosphate (KPi) buffer ( $\mathrm{pH} 7$ ), $20 \mu \mathrm{M}$ 4,4-dimethyl-4-silapentane-1-sulfonic acid used as a chemical shift reference in NMR, and containing $10 \% \mathrm{D}_{2} \mathrm{O}$. Concentrations of $\mathrm{KPi}$ and $\mathrm{KCl}$ varied to generate varying salt conditions, including "low salt" (1 mM KPi $+1 \mathrm{mM} \mathrm{KCl})$, "moderate salt" (10 mM KPi + $10 \mathrm{mM} \mathrm{KCl})$ and "physiological Salt” (10 mM KPi + $130 \mathrm{mM}$ $\mathrm{KCl})$.

\section{NMR spectroscopy}

Spectra were obtained on a $600 \mathrm{MHz}$ Bruker spectrometer (Billerica, MA U.S.A.) at a temperature of $25^{\circ} \mathrm{C}$ using a jump-and-return type water suppression pulse sequence (282). Samples were annealed prior to recording of NMR spectra by heating to $100^{\circ} \mathrm{C}$ for 2 minutes and allowed to cool slowly at room temperature. Chemical shifts were internally referenced with D.S.S.

\section{CD spectroscopy}

Spectra were recorded on a JASCO-815 spectropolarimeter (Tokyo, Japan). Samples contained a DNA strand concentration of $4-5 \mu \mathrm{M}$. Spectra were recorded at $20^{\circ} \mathrm{C}$ in the range of $220-320$ $\mathrm{nm}$ and averaged over 10 scans. Spectra were normalized through subtracting by the CD absorbance at $320 \mathrm{~nm}$ and dividing by concentration of the sample determined by concurrent UV absorption measurements. Samples were annealed prior to recording of CD spectra by heating to $100^{\circ} \mathrm{C}$ and allowed to cool slowly at room temperature.

\section{CD melting experiments}

CD melting experiments were performed for the unmodified sequence and for sequences containing multiple N2-modifications. Samples contained 4-5 $\mu \mathrm{M}$ DNA by strand concentration in $1 \mathrm{mM} \mathrm{KCl}$ and $1 \mathrm{mM} \mathrm{KPi}$. Samples were heated to $95^{\circ} \mathrm{C}\left(98^{\circ} \mathrm{C}\right.$ for N2-Ball-with-Hair sample) and cooled to $15^{\circ} \mathrm{C}$ at a rate of $0.2^{\circ} \mathrm{C} / \mathrm{min}$. Samples were then heated in a similar manner. Data was recorded every $0.5^{\circ} \mathrm{C}$ at $290 \mathrm{~nm}$ and corrected by subtraction at $320 \mathrm{~nm}$. Normalized melting curves are used to determine the melting temperature $\left(T_{m}\right)$ of modified sequences (270), which is taken as an average of the heating and cooling curves. Hysteresis in a heating/cooling cycle was observed to be below $2{ }^{\circ} \mathrm{C}$ over all samples.

\section{UV melting experiments}

Melting experiments were performed on a JASCO V-650 UV-visible spectrophotometer (Tokyo, Japan). Samples containing $\sim 5 \mu \mathrm{M}$ DNA strand concentration were first heated to $83^{\circ} \mathrm{C}$ and cooled to $14^{\circ} \mathrm{C}$ at a rate of $0.2^{\circ} \mathrm{C} / \mathrm{min}$. The absorbance was sampled every $0.5^{\circ} \mathrm{C}$ at $295 \mathrm{~nm}$ and $320 \mathrm{~nm}$. At the end of the cooling cycle, samples were held for 10 minutes before being heated back to $83^{\circ} \mathrm{C}$ at the same rate. Melting curves were normalized first by subtraction at $320 \mathrm{~nm}$ and then further processed to create fraction folded curves (258). Melting temperatures $\left(\mathrm{T}_{\mathrm{m}}\right)$ reported represent an average over heating and cooling cycles. Hysteresis of $<2{ }^{\circ} \mathrm{C}$ (generally $<$ $1.5^{\circ} \mathrm{C}$ ) was observed over all sequences.

\section{Modeling}

Models of Hex-3 and N2-Ball-with-Hair structures were built based on a NMR model of the unmodified (3+1) sequence (PDB ID 2GKU) (28). Guanine bases containing N2-6-amino-hexyl 
modifications in a straight orientation were fit to guanine bases within the NMR structure. The dihedral angles around the connection of the guanine base to the N2-6-amino-hexyl functional group (Guanine(C2)-Guanine(N2)-Hex(C1)-Hex(C2)) were then manually varied to project the N2-6-amino-hexyl into the groove. Illustrative models were visualized in Pymol. 


\section{Appendix III - PDB Survey}

Table A3-1. Summary and statistics of crystallographic structures cataloged in Chapter 2.

\begin{tabular}{|c|c|c|c|c|c|c|c|c|c|}
\hline \multirow{3}{*}{ PDB } & \multirow{3}{*}{ Sequence } & \multirow{3}{*}{ Description } & \multirow{3}{*}{$\begin{array}{l}\text { Res. } \\
(\AA)\end{array}$} & \multicolumn{6}{|c|}{ Statistics of Rotation (deg) / Separation ( $(\AA)$} \\
\hline & & & & \multicolumn{3}{|c|}{ Core Stacking } & \multicolumn{3}{|c|}{ Interface Stacking } \\
\hline & & & & Type & $\mathrm{N}$ & $\operatorname{Mean}(\sigma)$ & Type & $\mathrm{N}$ & $\operatorname{Mean}(\sigma)$ \\
\hline 1KF1 & $\begin{array}{l}\mathrm{d}\left[\mathrm{AG}_{3}\left(\mathrm{~T}_{2} \mathrm{~A}\right.\right. \\
\left.\left.\mathrm{G}_{3}\right)_{3}\right]\end{array}$ & $\begin{array}{lr}\text { Telomeric } & \text { DNA } \\
\text { Intramolecular Parallel } \\
\text { Propeller Quadruplex } \\
-\mathrm{K}^{+}\end{array}$ & 2.10 & $A / A$ & 8 & $\begin{array}{c}61.7(0.9) \\
3.42(0.08)\end{array}$ & $\begin{array}{c}5^{\prime}-5^{\prime} \\
5 / 6 \text {-ring }\end{array}$ & 2 & $\begin{array}{c}56.2(1.9) \\
3.38(0.05)\end{array}$ \\
\hline $1 \mathrm{~K} 8 \mathrm{P}$ & $\begin{array}{l}\mathrm{d}\left[{ }^{\mathrm{Br}} \mathrm{UAG}_{3}{ }^{\mathrm{Br}}\right. \\
\left.\mathrm{UTAG}_{3} \mathrm{~T}\right]_{2}\end{array}$ & $\begin{array}{lr}\text { Telomeric } & \text { DNA } \\
\text { Bimolecular Parallel } \\
\text { Propeller Quadruplex } \\
-\mathrm{K}^{+}\end{array}$ & 2.40 & $A / A$ & 8 & $\begin{array}{c}60.2(3.6) \\
3.32(0.06)\end{array}$ & - & - & - \\
\hline $2 \mathrm{HRI}$ & $\begin{array}{l}\mathrm{d}\left[\mathrm{TAG}_{3} \mathrm{~T}_{2} \mathrm{~A}\right. \\
\left.\mathrm{G}_{3}\right]_{2}\end{array}$ & 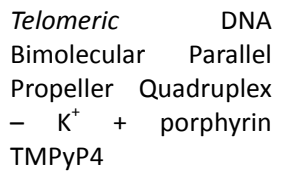 & 2.09 & $\mathrm{~A} / \mathrm{A}$ & 8 & $\begin{array}{c}63.4(2.7) \\
3.36(0.05)\end{array}$ & $\begin{array}{l}3^{\prime}-3^{\prime} \\
6 \text {-ring }\end{array}$ & 2 & $\begin{array}{c}46.0(0.9) \\
3.43(0.01)\end{array}$ \\
\hline $3 \mathrm{CCO}$ & $\begin{array}{l}\mathrm{d}\left[\mathrm{TAG}_{3} \mathrm{~T}_{2} \mathrm{~A}\right. \\
\left.\mathrm{G}_{3} \mathrm{~T}\right]_{2}\end{array}$ & 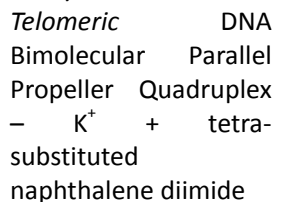 & 2.20 & $A / A$ & 4 & $\begin{array}{l}63.0(10.1) \\
3.42(0.15)\end{array}$ & $\begin{array}{l}5^{\prime}-5^{\prime} \\
\text { 5-ring }\end{array}$ & 1 & $\begin{array}{l}86.5(-) \\
3.46(-)\end{array}$ \\
\hline $3 C D M$ & $\begin{array}{l}\mathrm{d}\left[\mathrm{TA}\left(\mathrm{G}_{3} \mathrm{~T}_{2}\right.\right. \\
\left.\mathrm{A})_{3} \mathrm{G}_{3}\right]\end{array}$ & $\begin{array}{l}\text { Telomeric } \quad \text { DNA } \\
\text { Intramolecular Parallel } \\
\text { Propeller Quadruplex } \\
-\quad \mathrm{K}^{+}+\text {tetra- } \\
\text { substituted } \\
\text { naphthalene diimide }\end{array}$ & 2.10 & $A / A$ & 16 & $\begin{array}{c}62.3(2.3) \\
3.36(0.06)\end{array}$ & - & - & - \\
\hline 3CE5 & $\begin{array}{l}\mathrm{d}\left[\mathrm{TAG}_{3} \mathrm{~T}_{2} \mathrm{~A}\right. \\
\left.\mathrm{G}_{3} \mathrm{~T}\right]_{2}\end{array}$ & $\begin{array}{l}\text { Telomeric DNA } \\
\text { Bimolecular Parallel } \\
\text { Propeller Quadruplex } \\
-\mathrm{K}^{+}+\text {BRACO-19 }\end{array}$ & 2.50 & $A / A$ & 8 & $\begin{array}{c}63.1(1.2) \\
3.46(0.11)\end{array}$ & - & - & - \\
\hline $3 Q C R$ & $\begin{array}{l}\mathrm{d}\left[\mathrm{TAG}_{3} \mathrm{~T}_{2} \mathrm{~A}\right. \\
\left.\mathrm{G}_{3}\right]_{2}\end{array}$ & $\begin{array}{lr}\text { Telomeric DNA } \\
\text { Bimolecular Parallel } \\
\text { Propeller Quadruplex } \\
-\mathrm{K}^{+}+\text {acridine ligand }\end{array}$ & 3.2 & $A / A$ & 4 & $\begin{array}{c}60.6(0.7) \\
3.20(0.12)\end{array}$ & - & - & - \\
\hline 3IBK & $\begin{array}{l}\mathrm{r}\left[{ }^{5 \mathrm{Br}} \mathrm{UAG}_{3}\right. \\
\left.\mathrm{U}_{2} \mathrm{AG}_{3} \mathrm{U}\right]_{2}\end{array}$ & $\begin{array}{lr}\text { Telomeric } & \text { RNA } \\
\text { Bimolecular Parallel } \\
\text { Propeller Quadruplex } \\
-\mathrm{K}^{+}\end{array}$ & 2.20 & $A / A$ & 8 & $\begin{array}{c}60.9(4.9) \\
3.40(0.08)\end{array}$ & - & - & - \\
\hline $3 \mathrm{MIJ}$ & $\begin{array}{l}\mathrm{r}\left[\mathrm{UAG}_{3} \mathrm{U}_{2}\right. \\
\left.\mathrm{AG}_{3} \mathrm{U}\right]_{2}\end{array}$ & $\begin{array}{lr}\text { Telomeric } & \text { RNA } \\
\text { Bimolecular Parallel } \\
\text { Propeller Quadruplex } \\
-\mathrm{K}^{+}+\text {acridine ligand }\end{array}$ & 2.60 & $A / A$ & 4 & $\begin{array}{c}63.6(4.1) \\
3.40(0.11)\end{array}$ & - & - & - \\
\hline $2 \mathrm{AVH}$ & $\mathrm{d}\left[\mathrm{G}_{4} \mathrm{~T}_{3} \mathrm{G}_{4}\right]_{2}$ & $\begin{array}{l}\text { DNA Bimolecular }(2+2 \\
\uparrow \bullet \downarrow \cdot \uparrow \cdot \downarrow) \\
\text { Edgewise quadruplex - } \\
\mathrm{K}^{+}\end{array}$ & 1.50 & $\mathrm{~A} / \mathrm{S}$ & 2 & $\begin{array}{c}81.8(1.6) \\
3.47(0.07) \\
\\
30.4(2.1) \\
3.66(0.05)\end{array}$ & - & - & - \\
\hline 2AVJ & $\begin{array}{l}\mathrm{d}\left[\mathrm{G}_{4}{ }^{\mathrm{Br}} \mathrm{UT}_{2}\right. \\
\left.\mathrm{G}_{4}\right]_{2}\end{array}$ & $\begin{array}{l}\text { DNA Bimolecular }(2+2 \\
\uparrow \cdot \downarrow \cdot \uparrow \cdot \downarrow) \text { and }(2+2 \\
\uparrow \cdot \uparrow \cdot \downarrow \cdot \downarrow) \\
\text { Edgewise quadruplex } \\
-\mathrm{K}^{+}\end{array}$ & 2.39 & $\mathrm{~A} / \mathrm{S}$ & 24 & $\begin{array}{c}83.2(2.5) \\
3.46(0.09) \\
\\
29.0(1.5) \\
3.51(0.08)\end{array}$ & $\begin{array}{l}\text { Mixed 5'- } \\
\quad 3^{\prime} \\
\text { Partial } \\
\text { 6-ring }\end{array}$ & 4 & $\begin{array}{c}26.2(1.5) \\
3.50(0.08)\end{array}$ \\
\hline
\end{tabular}




\begin{tabular}{|c|c|c|c|c|c|c|c|}
\hline $1 J P Q$ & $\begin{array}{l}\mathrm{d}\left[\mathrm{G}_{4}{ }^{\mathrm{Br}} \mathrm{UT}_{3}\right. \\
\left.\mathrm{G}_{4}\right]_{2}\end{array}$ & $\begin{array}{l}\text { Oxytricha } \\
\text { Bimolecular } \\
\uparrow \cdot \uparrow \cdot \downarrow \cdot \downarrow) \\
\text { quadruplex }-\mathrm{K}\end{array}$ & $\begin{array}{r}\text { DNA } \\
(2+2 \\
\text { Diagonal }\end{array}$ & 1.60 & $\mathrm{~A} / \mathrm{S}$ & 4 & $\begin{array}{c}83.0(2.0) \\
3.48(0.12)\end{array}$ \\
\hline 1JRN & $\mathrm{d}\left[\mathrm{G}_{4} \mathrm{~T}_{4} \mathrm{G}_{4}\right]_{2}$ & $\begin{array}{l}\text { Oxytricha } \\
\text { Bimolecular } \\
\uparrow \cdot \uparrow \cdot \downarrow \bullet \downarrow) \\
\text { quadruplex }-\mathrm{K}\end{array}$ & $\begin{array}{r}\text { DNA } \\
(2+2 \\
\text { Diagonal } \\
T^{+}\end{array}$ & 2.00 & $\mathrm{~A} / \mathrm{S}$ & 16 & $\begin{array}{c}29.1(0.9) \\
3.53(0.08)\end{array}$ \\
\hline $2 \mathrm{HBN}$ & $\mathrm{d}\left[\mathrm{G}_{4} \mathrm{~T}_{4} \mathrm{G}_{4}\right]_{2}$ & $\begin{array}{l}\text { Oxytricha } \\
\text { Bimolecular } \\
\uparrow \cdot \uparrow \cdot \downarrow \cdot \downarrow) \\
\text { quadruplex }-\mathrm{T}\end{array}$ & $\begin{array}{r}\text { DNA } \\
(2+2 \\
\text { Diagonal } \\
I^{+}\end{array}$ & 1.55 & $A / S$ & 16 & $\begin{array}{c}82.8(2.7) \\
3.49(0.12) \\
\\
29.2(1.9) \\
3.57(0.04)\end{array}$ \\
\hline $2 \mathrm{GWQ}$ & $\mathrm{d}\left[\mathrm{G}_{4} \mathrm{~T}_{4} \mathrm{G}_{4}\right]_{2}$ & $\begin{array}{l}\text { Oxytricha } \\
\text { Bimolecular } \\
\uparrow \cdot \uparrow \cdot \downarrow \cdot \downarrow) \\
\text { quadruplex }-\mathrm{K}\end{array}$ & $\begin{array}{r}\text { DNA } \\
(2+2 \\
\text { Diagonal }\end{array}$ & 2.00 & $A / S$ & 16 & $\begin{array}{c}83.1(1.0) \\
3.46(0.10) \\
\\
29.5(1.6) \\
3.54(0.07)\end{array}$ \\
\hline 2GWE & $\mathrm{d}\left[\mathrm{G}_{4} \mathrm{~T}_{4} \mathrm{G}_{4}\right]_{2}$ & $\begin{array}{l}\text { Oxytricha } \\
\text { Bimolecular } \\
\uparrow \cdot \uparrow \cdot \downarrow \cdot \downarrow) \\
\text { quadruplex }-\mathrm{K}\end{array}$ & $\begin{array}{r}\text { DNA } \\
(2+2 \\
\text { Diagonal }\end{array}$ & 2.20 & $\mathrm{~A} / \mathrm{S}$ & 48 & $\begin{array}{c}83.0(2.8) \\
3.44(0.13) \\
\\
29.3(1.6) \\
3.55(0.08)\end{array}$ \\
\hline $1 \mathrm{~L} 1 \mathrm{H}$ & $\mathrm{d}\left[\mathrm{G}_{4} \mathrm{~T}_{4} \mathrm{G}_{4}\right]_{2}$ & $\begin{array}{l}\text { Oxytricha } \\
\text { Bimolecular } \\
\uparrow \cdot \uparrow \cdot \downarrow \cdot \downarrow) \\
\text { quadruplex - } \\
\text { acridine ligand }\end{array}$ & $\begin{array}{r}\text { DNA } \\
(2+2 \\
\text { Diagonal } \\
\mathrm{K}^{+}+\end{array}$ & 1.75 & $A / S$ & 4 & $\begin{array}{c}83.1(2.1) \\
3.42(0.10) \\
\\
28.8(1.1) \\
3.56(0.07)\end{array}$ \\
\hline 3ЕМ2 & $\mathrm{d}\left[\mathrm{G}_{4} \mathrm{~T}_{4} \mathrm{G}_{4}\right]_{2}$ & $\begin{array}{l}\text { Oxytricha } \\
\text { Bimolecular } \\
\uparrow \bullet \uparrow \cdot \downarrow \cdot \downarrow) \\
\text { quadruplex } \\
\text { acridine ligand }\end{array}$ & $\begin{array}{r}\text { DNA } \\
(2+2 \\
\text { Diagonal } \\
-\mathrm{K}^{+}+\end{array}$ & 2.30 & $A / S$ & 8 & $\begin{array}{c}84.0(3.0) \\
3.42(0.09) \\
\\
28.7(1.6) \\
3.57(0.05)\end{array}$ \\
\hline 3EQW & $\mathrm{d}\left[\mathrm{G}_{4} \mathrm{~T}_{4} \mathrm{G}_{4}\right]_{2}$ & $\begin{array}{l}\text { Oxytricha } \\
\text { Bimolecular } \\
\uparrow \cdot \uparrow \cdot \downarrow \cdot \downarrow) \\
\text { quadruplex } \\
\text { acridine ligand }\end{array}$ & $\begin{array}{r}\text { DNA } \\
(2+2 \\
\text { Diagonal } \\
-\quad \mathrm{K}^{+}+\end{array}$ & 2.20 & $\mathrm{~A} / \mathrm{S}$ & 8 & $\begin{array}{c}83.9(2.6) \\
3.43(0.08) \\
\\
28.6(1.2) \\
3.53(0.06)\end{array}$ \\
\hline 3ERU & $\mathrm{d}\left[\mathrm{G}_{4} \mathrm{~T}_{4} \mathrm{G}_{4}\right]_{2}$ & $\begin{array}{l}\text { Oxytricha } \\
\text { Bimolecular } \\
\uparrow \cdot \uparrow \bullet \downarrow \bullet \downarrow) \\
\text { quadruplex } \\
\text { acridine ligand }\end{array}$ & $\begin{array}{r}\text { DNA } \\
(2+2 \\
\text { Diagonal } \\
-\quad \mathrm{K}^{+}+\end{array}$ & 2.00 & $\mathrm{~A} / \mathrm{S}$ & 8 & $\begin{array}{c}83.8(2.5) \\
3.43(0.08)\end{array}$ \\
\hline 3 ESO & $\mathrm{d}\left[\mathrm{G}_{4} \mathrm{~T}_{4} \mathrm{G}_{4}\right]_{2}$ & $\begin{array}{l}\text { Oxytricha } \\
\text { Bimolecular } \\
\uparrow \cdot \uparrow \cdot \downarrow \cdot \downarrow) \\
\text { quadruplex } \\
\text { acridine ligand }\end{array}$ & $\begin{array}{r}\text { DNA } \\
(2+2 \\
\text { Diagonal } \\
-\quad \mathrm{K}^{+}+\end{array}$ & 2.20 & $A / S$ & 4 & $\begin{array}{c}83.8(1.5) \\
3.46(0.06)\end{array}$ \\
\hline
\end{tabular}




\begin{tabular}{|c|c|c|c|c|c|c|c|c|c|}
\hline 3ET8 & $\mathrm{d}\left[\mathrm{G}_{4} \mathrm{~T}_{4} \mathrm{G}_{4}\right]_{2}$ & $\begin{array}{lr}\text { Oxytricha } & \text { DNA } \\
\text { Bimolecular } & (2+2 \\
\uparrow \bullet \uparrow \cdot \downarrow \bullet \downarrow) & \text { Diagonal } \\
\text { quadruplex }- & \mathrm{K}^{+}+ \\
\text {acridine ligand } & \end{array}$ & 2.45 & S/A & 8 & $\begin{array}{c}82.7(2.4) \\
3.52(0.09) \\
29.9(1.6) \\
3.59(0.08)\end{array}$ & - & - & - \\
\hline 3EUI & $\mathrm{d}\left[\mathrm{G}_{4} \mathrm{~T}_{4} \mathrm{G}_{4}\right]_{2}$ & $\begin{array}{lr}\text { Oxytricha } & \text { DNA } \\
\text { Bimolecular } & (2+2 \\
\uparrow \bullet \uparrow \cdot \downarrow \bullet \downarrow) & \text { Diagonal } \\
\text { quadruplex }- & \mathrm{K}^{+}+ \\
\text {acridine ligand } & \end{array}$ & 2.20 & $S / A$ & 16 & $\begin{array}{c}84.2(1.9) \\
3.47(0.10) \\
\\
30.3(0.7) \\
3.55(0.08)\end{array}$ & - & - & - \\
\hline 3EUM & $\mathrm{d}\left[\mathrm{G}_{4} \mathrm{~T}_{4} \mathrm{G}_{4}\right]_{2}$ & $\begin{array}{lr}\text { Oxytricha } & \text { DNA } \\
\text { Bimolecular } & (2+2 \\
\uparrow \cdot \uparrow \cdot \downarrow \bullet \downarrow) & \text { Diagonal } \\
\text { quadruplex }- & \mathrm{K}^{+}+ \\
\text {acridine ligand } & \end{array}$ & 1.78 & $A / S$ & 4 & $\begin{array}{c}83.5(1.9) \\
3.45(0.08) \\
\\
29.0(0.8) \\
3.49(0.04)\end{array}$ & - & - & - \\
\hline 3NYP & $\mathrm{d}\left[\mathrm{G}_{4} \mathrm{~T}_{4} \mathrm{G}_{4}\right]_{2}$ & $\begin{array}{lr}\text { Oxytricha } & \text { DNA } \\
\text { Bimolecular } & (2+2 \\
\uparrow \bullet \uparrow \cdot \downarrow \bullet \downarrow) & \text { Diagonal } \\
\text { quadruplex }- & \mathrm{K}^{+}+ \\
\text {acridine ligand } & \end{array}$ & 1.18 & $A / S$ & 8 & $\begin{array}{c}84.6(2.3) \\
3.60(0.07) \\
\\
28.1(1.1) \\
3.71(0.04)\end{array}$ & - & - & - \\
\hline 3NZ7 & $\mathrm{d}\left[\mathrm{G}_{4} \mathrm{~T}_{4} \mathrm{G}_{4}\right]_{2}$ & $\begin{array}{lr}\text { Oxytricha } & \text { DNA } \\
\text { Bimolecular } & (2+2 \\
\uparrow \bullet \uparrow \cdot \downarrow \bullet \downarrow) & \text { Diagonal } \\
\text { quadruplex }- & \mathrm{K}^{+}+ \\
\text {acridine ligand } & \end{array}$ & 1.10 & $A / S$ & 8 & $\begin{array}{c}83.8(2.0) \\
3.44(0.07) \\
\\
29.0(0.9) \\
3.56(0.03)\end{array}$ & - & - & - \\
\hline 2GW0 & $\mathrm{d}\left[\mathrm{TG}_{4} \mathrm{~T}\right]_{4}$ & $\begin{array}{l}\text { Tetramolecular parallel } \\
\text { DNA quadruplex }-\mathrm{Na}^{+} / \\
\mathrm{Ca}^{+2}\end{array}$ & 1.55 & $A / A$ & 24 & $\begin{array}{c}60.6(3.0) \\
3.35(0.06)\end{array}$ & $\begin{array}{l}5^{\prime}-5^{\prime} \\
5 \text {-ring }\end{array}$ & 4 & $\begin{array}{c}81.5(0.7) \\
3.49(0.04)\end{array}$ \\
\hline 244D & $\mathrm{d}\left[\mathrm{TG}_{4} \mathrm{~T}\right]_{4}$ & $\begin{array}{l}\text { Tetramolecular parallel } \\
\text { DNA quadruplex }-\mathrm{Na}^{+}\end{array}$ & 1.20 & $A / A$ & 48 & $\begin{array}{c}60.6(2.8) \\
3.41(0.05)\end{array}$ & $\begin{array}{l}5^{\prime}-5^{\prime} \\
5 \text {-ring }\end{array}$ & 8 & $\begin{array}{c}83.0(0.4) \\
3.50(0.02)\end{array}$ \\
\hline 352D & $\mathrm{d}\left[\mathrm{TG}_{4} \mathrm{~T}\right]_{4}$ & $\begin{array}{l}\text { Tetramolecular parallel } \\
\text { DNA quadruplex }-\mathrm{Na}^{+}\end{array}$ & 0.95 & $A / A$ & 48 & $\begin{array}{c}60.9(3.1) \\
3.34(0.05)\end{array}$ & $\begin{array}{l}5^{\prime}-5^{\prime} \\
5 \text {-ring }\end{array}$ & 8 & $\begin{array}{l}82.7(0.5) \\
3.42(0.03)\end{array}$ \\
\hline $100 \mathrm{~K}$ & $\mathrm{~d}\left[\mathrm{TG}_{4} \mathrm{~T}\right]_{4}$ & $\begin{array}{l}\text { Tetramolecular parallel } \\
\text { DNA quadruplex }-\mathrm{Na}^{+} \\
+ \text {daunomycin ligand }\end{array}$ & 1.17 & $\mathrm{~A} / \mathrm{A}$ & 12 & $\begin{array}{l}59.1(4.2) \\
3.27(0.04)\end{array}$ & - & - & - \\
\hline $1 S 45$ & $\mathrm{~d}\left[\mathrm{TG}_{4} \mathrm{~T}\right]_{4}$ & $\begin{array}{l}\text { Tetramolecular parallel } \\
\text { DNA quadruplex - } \\
\mathrm{Na}^{+} / \mathrm{TI}^{+}\end{array}$ & 2.20 & $A / A$ & 24 & $\begin{array}{l}61.8(2.6) \\
3.42(0.09)\end{array}$ & $\begin{array}{l}5^{\prime}-5^{\prime} \\
5-\text {-ring }\end{array}$ & 4 & $\begin{array}{c}84.5(0.7) \\
3.59(0.02)\end{array}$ \\
\hline 1547 & $\mathrm{~d}\left[\mathrm{TG}_{4} \mathrm{~T}\right]_{4}$ & $\begin{array}{l}\text { Tetramolecular parallel } \\
\text { DNA quadruplex - } \\
\mathrm{Na}^{+} / \mathrm{TI}^{+}\end{array}$ & 2.50 & $\mathrm{~A} / \mathrm{A}$ & 36 & $\begin{array}{l}62.5(3.3) \\
3.45(0.16)\end{array}$ & $\begin{array}{l}5^{\prime}-5^{\prime} \\
5-\text {-ring }\end{array}$ & 4 & $\begin{array}{l}85.1(1.8) \\
3.60(0.07)\end{array}$ \\
\hline $1 \mathrm{~V} 3 \mathrm{P}$ & $\begin{array}{l}\mathrm{d}\left[\mathrm{G}^{51} \mathrm{CGA}\right. \\
\mathrm{GAGC}]_{4}\end{array}$ & $\begin{array}{l}\text { Octamolecular } \\
\text { quadruplex }-\mathrm{K}^{+}\end{array}$ & 2.30 & - & - & - & $\begin{array}{c}5^{\prime}-5^{\prime} \\
5 / 6-\text { ring }\end{array}$ & 1 & $\begin{array}{l}57.1(-) \\
3.47(-)\end{array}$ \\
\hline 1P79 & $\begin{array}{l}{\left[r\left(U_{G U G}\right.\right.} \\
U)]_{4}\end{array}$ & $\begin{array}{l}\text { Tetramolecular parallel } \\
\text { Bulged } \\
\text { quadruplex }-\mathrm{K}^{+}\end{array}$ & 1.10 & $\mathrm{~A} / \mathrm{A}$ & 1 & $\begin{array}{l}77.7(-) \\
3.40(-) \\
61.9(-) \\
3.41(-)\end{array}$ & - & - & - \\
\hline
\end{tabular}




\begin{tabular}{|c|c|c|c|c|c|c|c|c|c|}
\hline 2AWE & $\begin{array}{l}\mathrm{r}\left[\mathrm{U}^{8 \mathrm{Br}} \mathrm{GGU}\right. \\
\mathrm{GU}]_{4}\end{array}$ & $\begin{array}{l}\text { Tetramolecular parallel } \\
\text { Bulged } \\
\text { quadruplex }-\mathrm{K}^{+}\end{array}$ & 2.10 & $\begin{array}{l}A / A \\
B^{b}\end{array}$ & 8 & $\begin{array}{c}82.2(0.8) \\
3.57(0.04) \\
35.7(0.9) \\
3.53(0.06)\end{array}$ & - & - & - \\
\hline 2GRB & $\begin{array}{l}\mathrm{r}\left[{ }^{5 \mathrm{Br}} \mathrm{UGIG}\right. \\
\mathrm{GU}]_{4}\end{array}$ & $\begin{array}{l}\text { Tetramolecular parallel } \\
\text { RNA quadruplex }-\mathrm{K}^{+}\end{array}$ & 1.40 & $\mathrm{~A} / \mathrm{A}$ & 8 & $\begin{array}{l}65.5(0.9) \\
3.52(0.04)\end{array}$ & - & - & - \\
\hline $1 \mathrm{~J} 6 \mathrm{~S}$ & $\begin{array}{l}{\left[r\left({ }^{B r} U G A G\right.\right.} \\
\left.\left.{ }_{2} U\right)\right]_{4}\end{array}$ & $\begin{array}{l}\text { Tetramolecular parallel } \\
\text { RNA quadruplex }-\mathrm{Ba}^{2+}\end{array}$ & 1.40 & $\mathrm{~A} / \mathrm{A}$ & 4 & $\begin{array}{l}65.7(0.5) \\
3.46(0.05)\end{array}$ & - & - & - \\
\hline $1 J 8 G$ & $r\left[U_{4} U\right]_{4}$ & $\begin{array}{l}\text { Tetramolecular parallel } \\
\text { RNA quadruplex }-\mathrm{Sr}^{2+}\end{array}$ & 0.61 & $\mathrm{~A} / \mathrm{A}$ & 12 & $\begin{array}{c}60.6(3.9) \\
3.31(0.04)\end{array}$ & $\begin{array}{l}5^{\prime}-5^{\prime} \\
6-\text {-ring }\end{array}$ & 2 & $\begin{array}{c}37.2(0.7) \\
3.39(0.00)\end{array}$ \\
\hline $1 M D G$ & $\begin{array}{l}{\left[r(U) d\left({ }^{B r} G\right)\right.} \\
\left.r\left(A_{2} U\right)\right]_{4}\end{array}$ & $\begin{array}{l}\text { Tetramolecular parallel } \\
\text { RNA quadruplex }-\mathrm{Na}^{+}\end{array}$ & 1.50 & $\mathrm{~A} / \mathrm{A}$ & 1 & $\begin{array}{l}64.5(-) \\
3.39(-)\end{array}$ & - & - & - \\
\hline $204 \mathrm{~F}$ & $\mathrm{~d}\left[\mathrm{TG}_{4} \mathrm{~T}\right]_{4}$ & $\begin{array}{l}\text { Tetramolecular parallel } \\
\text { DNA quadruplex }-\mathrm{Na}^{+}\end{array}$ & 1.50 & $\mathrm{~A} / \mathrm{A}$ & 24 & $\begin{array}{c}61.2(2.6) \\
3.42(0.06)\end{array}$ & $\begin{array}{l}5 '-5^{\prime} \\
5 \text {-ring }\end{array}$ & 4 & $\begin{array}{c}82.7(0.2) \\
3.63(0.03)\end{array}$ \\
\hline
\end{tabular}

G-quadruplex structures included in this work were obtained by searching the Protein Data Bank using the following keywords: quadruplex(es); tetrad(s); tetraplex(es); G4. PDB Entries 1D59 and 1JB7 were not examined in this work. Statistics presented are for experimental stacked guanines pairs from a single PDB compared to model stacked guanine pairs.

${ }^{a}$ PDB Entries from work not yet published.

${ }^{\mathrm{b}}$ Anti/Anti Stacking present with a one residue bulge. 
Table A3-2. Summary and statistics of NMR G-quadruplex structures cataloged in Chapter 2.

\begin{tabular}{|c|c|c|c|c|c|c|c|c|}
\hline \multirow{3}{*}{ PDB } & \multirow{3}{*}{ Sequence } & \multirow{3}{*}{ Description } & \multicolumn{6}{|c|}{ Statistics of Rotation (deg) / Seperation $(\AA)$} \\
\hline & & & \multicolumn{3}{|c|}{ Core Stacking } & \multicolumn{3}{|c|}{ Interface Stacking } \\
\hline & & & Type & $\mathrm{N}$ & $\operatorname{Mean}(\sigma)$ & Type & $\mathrm{N}$ & $\operatorname{Mean}(\sigma)$ \\
\hline 139D & $\mathrm{d}\left[\mathrm{T}_{2} \mathrm{G}_{4} \mathrm{~T}\right]_{4}$ & $\begin{array}{l}\text { DNA Intermolecular } \\
\text { Parallel Quadruplex - } \\
\mathrm{K}^{+}\end{array}$ & $\mathrm{A} / \mathrm{A}$ & 12 & $\begin{array}{l}60.1(5.0) \\
3.17(0.08)\end{array}$ & - & - & - \\
\hline 143D & $\mathrm{d}\left[\mathrm{AG}_{3}\left(\mathrm{~T}_{2} \mathrm{AG}_{3}\right)_{3}\right]$ & $\begin{array}{l}\text { DNA Intramolecular } \\
\text { Telomeric } \quad(2+2 \\
\uparrow \cdot \downarrow \cdot \uparrow \cdot \downarrow) \text { Quadruplex } \\
-\mathrm{Na}^{+}\end{array}$ & S/A & 4 & $\begin{array}{l}79.0(5.3) \\
3.45(0.12)\end{array}$ & - & - & - \\
\hline 148D & $\begin{array}{l}\mathrm{d}\left[\mathrm{G}_{2} \mathrm{~T}_{2} \mathrm{G}_{2} \mathrm{TGTG}_{2}\right. \\
\left.\mathrm{T}_{2} \mathrm{G}_{2}\right]_{2}\end{array}$ & $\begin{array}{l}\text { DNA Intermolecular } \\
\text { Thrombin aptamer } \\
(2+2 \quad \uparrow \cdot \downarrow \cdot \uparrow \cdot \downarrow) \\
\text { Quadruplex }-\mathrm{K}^{+}\end{array}$ & S/A & 4 & $\begin{array}{l}86.4(3.8) \\
3.55(0.57)\end{array}$ & - & - & - \\
\hline 156D & $\mathrm{d}\left[\mathrm{G}_{4} \mathrm{~T}_{4} \mathrm{G}_{4}\right]_{2}$ & $\begin{array}{l}\text { Oxytricha } \\
\text { Intermolecular DNA } \\
(2+2 \quad \uparrow \cdot \uparrow \cdot \downarrow \cdot \downarrow) \\
\text { Quadruplex }-\mathrm{K}^{+}\end{array}$ & $\mathrm{A} / \mathrm{S}$ & 4 & $\begin{array}{l}85.7(4.8) \\
3.38(0.37) \\
\\
31.7(8.4) \\
3.39(0.32)\end{array}$ & - & - & - \\
\hline & & & S/A & 4 & $\begin{array}{l}84.8(2.0) \\
3.38(0.25)\end{array}$ & & & \\
\hline 186D & $\mathrm{d}\left[\left(\mathrm{T}_{2} \mathrm{G}_{4}\right)_{4}\right]$ & $\begin{array}{l}\text { Oxytricha } \\
\text { Intarmolecular DNA } \\
(3+1) \text { Quadruplex - } \\
\mathrm{Na}+\end{array}$ & $\mathrm{A} / \mathrm{A}$ & 3 & $\begin{array}{l}63.9(10.8) \\
3.51(0.28)\end{array}$ & - & - & - \\
\hline & & & $\mathrm{S} / \mathrm{S}$ & 1 & $\begin{array}{l}15.1(-) \\
3.28(-)\end{array}$ & & & \\
\hline $1 \mathrm{~A} 8 \mathrm{~N}$ & $\mathrm{~d}\left[\mathrm{G}_{3} \mathrm{CT}_{4} \mathrm{G}_{3} \mathrm{C}\right]_{2}$ & $\begin{array}{l}\text { Human } \\
\text { Chromosomal repeat } \\
\text { DNA }(2+2 \uparrow \cdot \uparrow \cdot \downarrow \cdot \downarrow) \\
\text { Quadruplex }-\mathrm{Na}^{+}\end{array}$ & S/A & 4 & $\begin{array}{l}88.3(1.5) \\
3.46(0.02)\end{array}$ & - & - & - \\
\hline $1 \mathrm{~A} 8 \mathrm{~W}$ & $\mathrm{~d}\left[\mathrm{G}_{3} \mathrm{CT}_{4} \mathrm{G}_{3} \mathrm{C}\right]_{2}$ & $\begin{array}{l}\text { DNA }(2+2 \quad \uparrow \cdot \uparrow \cdot \downarrow \cdot \downarrow) \\
\text { Quadruplex }-\mathrm{K}+\end{array}$ & S/A & 4 & $\begin{array}{l}81.6(0.5) \\
3.61(0.03)\end{array}$ & - & - & - \\
\hline $1 \mathrm{AFF}$ & $\mathrm{d}\left[\mathrm{T}_{2} \mathrm{AG}_{3}\right]_{4}$ & $\begin{array}{l}\text { Tetrameric DNA } \\
\text { Quadruplex - }\end{array}$ & S/A & 4 & $\begin{array}{l}84.0(10.1) \\
3.76(0.04)\end{array}$ & - & - & - \\
\hline \multirow{3}{*}{ 2GKU } & \multirow{3}{*}{$\begin{array}{l}\mathrm{d}\left[\mathrm{T}_{2}\left(\mathrm{G}_{3} \mathrm{~T}_{2} \mathrm{~A}\right)_{3}\right. \\
\left.\mathrm{G}_{3} \mathrm{~A}\right]\end{array}$} & \multirow{3}{*}{$\begin{array}{l}\text { Telomeric DNA } \\
(3+1) \text { Quadruplex - } \\
\text { K+ }\end{array}$} & $\mathrm{A} / \mathrm{A}$ & 3 & $\begin{array}{l}\text { 61.1(6.4) } \\
3.38(0.09)\end{array}$ & & & \\
\hline & & & $\mathrm{S} / \mathrm{S}$ & 1 & $\begin{array}{l}30.1(-) \\
3.55(-)\end{array}$ & - & - & - \\
\hline & & & S/A & 4 & $\begin{array}{l}82.3(3.0) \\
3.57(0.18)\end{array}$ & & & \\
\hline \multirow{3}{*}{ 2KZD } & \multirow{3}{*}{$\begin{array}{l}\mathrm{d}\left[\mathrm{AG}_{3} \mathrm{IAG}_{4} \mathrm{CTG}_{3}\right. \\
\left.\mathrm{AG}_{3} \mathrm{C}\right]\end{array}$} & \multirow{3}{*}{$\begin{array}{l}\text { DNA } \quad(3+1) \\
\text { Quadruplex }-\mathrm{K}^{+}\end{array}$} & $\mathrm{A} / \mathrm{A}$ & 3 & $\begin{array}{l}59.9(3.1) \\
3.33(0.14)\end{array}$ & & & \\
\hline & & & $\mathrm{S} / \mathrm{S}$ & 1 & $\begin{array}{l}28.2(-) \\
3.93(-)\end{array}$ & - & - & - \\
\hline & & & S/A & 4 & $\begin{array}{l}85.8(2.1) \\
3.93(0.15)\end{array}$ & & & \\
\hline 2KAZ & $\begin{array}{l}\mathrm{d}\left[\mathrm{G}_{3} \mathrm{ACGTAGT}\right. \\
\left.\mathrm{G}_{3}\right]_{2}\end{array}$ & $\begin{array}{l}\text { DNA }(2+2 \uparrow \uparrow \cdot \uparrow \cdot \downarrow \cdot \downarrow) \\
\text { Diagonal Quadruplex } \\
-\mathrm{K}^{+}\end{array}$ & $\mathrm{A} / \mathrm{A}$ & 2 & $\begin{array}{l}64.4(1.74) \\
3.37(0.12) \\
\\
27.9(2.2) \\
3.53(0.21)\end{array}$ & - & - & - \\
\hline
\end{tabular}




\begin{tabular}{|c|c|c|c|c|c|c|c|c|}
\hline & & & $\mathrm{S} / \mathrm{A}$ & 4 & $\begin{array}{l}85.9(2.6) \\
3.42(0.08)\end{array}$ & & & \\
\hline 1MYQ & $\mathrm{d}\left[(\mathrm{GGA})_{4}\right]$ & $\begin{array}{l}\text { DNA Parallel } \\
\text { Propeller } \\
\text { Quadruplex }-\mathrm{K}+\end{array}$ & - & - & - & $\begin{array}{l}\text { 5'-5' } \\
\text { 5/6-ring }\end{array}$ & 4 & $\begin{array}{l}60.1(7.5) \\
3.26(0.23)\end{array}$ \\
\hline 1MY9 & $\begin{array}{l}\mathrm{r}\left[\mathrm{G}_{2} \mathrm{AG}_{2} \mathrm{U}_{4} \mathrm{G}_{2} \mathrm{AG}\right. \\
2]\end{array}$ & $\begin{array}{l}\text { RNA Parallel } \\
\text { Propeller } \\
\text { Quadruplex }-\mathrm{K}+\end{array}$ & - & - & - & $\begin{array}{l}\text { 5'-5' } \\
\text { 5-ring } \\
\text { 5/6-ring }\end{array}$ & 3 & $\begin{array}{l}84.2(2.4) \\
3.00(0.19) \\
71.3(-) \\
3.93(-)\end{array}$ \\
\hline $1 \mathrm{EEG}$ & $\mathrm{d}\left[(\mathrm{GGA})_{2}\right]_{2}$ & $\begin{array}{l}\text { DNA Biomolecular } \\
\text { Parallel Propeller } \\
\text { Quadruplex }-\mathrm{Na}^{+}\end{array}$ & - & - & - & $\begin{array}{l}5^{\prime} \leftrightarrow 5^{\prime} \\
\text { ND }\end{array}$ & - & - \\
\hline $10 Z 8$ & $\mathrm{~d}\left[(\mathrm{GGA})_{8}\right]$ & $\begin{array}{l}\text { DNA Linked Dimeric } \\
\text { Parallel Propeller } \\
\text { Quadruplex } \\
\mathrm{Na}+/ \mathrm{K}+\end{array}$ & - & - & - & $\begin{array}{l}\text { Linked } \\
\text { 5'↔5' } \\
\text { 5/6-ring }\end{array}$ & 4 & $\begin{array}{c}61.7(5.0) \\
3.57(0.50)\end{array}$ \\
\hline 1JJP & $\begin{array}{l}\mathrm{d}\left[\mathrm{G}_{3} \mathrm{AG}_{2} \mathrm{~T}_{3} \mathrm{G}_{3} \mathrm{AT}\right. \\
]_{2}\end{array}$ & $\begin{array}{ll}\text { DNA } & \text { Interlocked } \\
\text { Dimeric } & \text { Quadruplex } \\
-\mathrm{Na}^{+} & \end{array}$ & - & - & - & $\begin{array}{l}\text { Locked } \\
\text { 5-ring }\end{array}$ & - & - \\
\hline 2RQJ & $\mathrm{r}\left[(\mathrm{GGA})_{4}\right]$ & $\begin{array}{l}\text { RNA Parallel } \\
\text { Propeller } \\
\text { Quadruplex }-\mathrm{K}+\end{array}$ & - & - & - & $\begin{array}{l}\text { 5'-5' } \\
\text { 5-ring } \\
\text { 5/6-ring }\end{array}$ & 2 & $\begin{array}{l}77.2(0.1) \\
3.26(0.11) \\
68.5(0.2) \\
3.32(0.06)\end{array}$ \\
\hline 1Y8D & $\begin{array}{l}\mathrm{d}\left[\mathrm{G}_{4} \mathrm{TG}_{3} \mathrm{AG}_{2} \mathrm{AG}_{3}\right. \\
\mathrm{T}]_{2}\end{array}$ & $\begin{array}{ll}\text { DNA } & \text { Interlocked } \\
\text { Dimeric } & \text { Quadruplex } \\
-\mathrm{K}+ & \end{array}$ & - & - & - & $\begin{array}{l}\text { Locked } \\
\text { 5-ring }\end{array}$ & - & - \\
\hline 2LE6 & $\mathrm{d}\left[\mathrm{GIGT}\left(\mathrm{G}_{3} \mathrm{~T}\right)_{3}\right]$ & $\begin{array}{l}\text { DNA Parallel } \\
\text { Propeller } \\
\text { Quadruplex } \\
\text { - K+ }\end{array}$ & - & - & - & $\begin{array}{l}\text { 5'-5' } \\
\text { 5/6-ring }\end{array}$ & 4 & $\begin{array}{l}56.8(1.94) \\
3.37(0.04)\end{array}$ \\
\hline 2WCN & $\begin{array}{l}\mathrm{d}\left[\mathrm{G}\left({ }^{\mathrm{LNA}} \mathrm{G}\right) \mathrm{G}\left({ }^{\mathrm{LNA}}\right.\right. \\
\mathrm{G}) \mathrm{T}_{4} \mathrm{G}\left({ }^{\mathrm{LNA}} \mathrm{G}\right) \mathrm{G}\left({ }^{\mathrm{L}}\right. \\
\left.\left.{ }^{\mathrm{NA}} \mathrm{G}\right)\right]\end{array}$ & $\begin{array}{l}\text { DNA V4 folded } \\
\text { Quadruplex }-\mathrm{K}+\end{array}$ & - & - & - & $\begin{array}{l}\text { Locked } \\
\text { P 6-ring }\end{array}$ & - & - \\
\hline
\end{tabular}

G-quadruplex structures included in this work cover a range of different folding topologies and include structures in which stacked guanine tetrads are presented at the interface between monomer G-quadruplexes. Statistics presented are for experimental stacked guanines pairs from a single PDB compared to model stacked guanine pairs. 